\title{
Synthesis and investigation of frustrated Honeycomb lattice iridates and rhodates
}

\author{
Dissertation \\ for the award of the degree \\ "Doctor of Philosophy" \\ (Ph.D.) \\ of the Georg-August-Universität Göttingen \\ within the doctoral program ProPhys \\ of the Georg-August University School of Science (GAUSS)
}

by

Soham Manni

from Bhatpara, India

Göttingen, 2014 


\section{Thesis committee:}

Prof. Dr. Philipp Gegenwart, Experimentalphysik VI, Institut für Physik, University of Augsburg, Germany

Prof. Dr. Andreas Honecker, Institut für Theoretische Physik, Universität Göttingen, Germany

\section{Members of the examination board:}

Referee: Prof. Dr. Philipp Gegenwart, Experimentalphysik VI, Institut für Physik, University of Augsburg, Germany

Co-referee: Prof. Dr. Angela Rizzi, IV Physik Institut, Universität Göttingen, Germany Co-referee: Dr. Yogesh Singh, Indian Institute of Science Education and Research Mohali, India

\section{Other Members of the examination board:}

Prof. Dr.Andreas Honecker , Institut für Theoretische Physik, Universität Göttingen, Germany

Prof. Dr. Vasily Moshnyega, I Physik Institut, Universität Göttingen, Germany

Prof. Dr. Markus Münzenberg, Institut für Physik, Universität Greifswald, Germany

Prof. Dr. Reiner. G. Ulbrich, IV Physik Institut, Universität Göttingen, Germany

Date of the oral examination : $27^{\text {th }}$ June, 2014. 


\section{Acknowledgements}

I would like to begin by acknowledging the great support and help from my supervisor Prof. Dr. Philipp Gegenwart. From the very beginning of this thesis, his strong interest and quest to explore this new field have supplied a lot of positive energy and courage within me even in the very difficult times of the project. I would like to thank him for making this project successful with his support as well as by organizing many fruitful collaborations. I would also like to acknowledge my thesis committee member Prof. Dr. Andreas Honecker for his many helpful supports until the submission of the thesis and Prof. Dr. Angela Rizzi for her last hour kind agreement to be a co-referee of this thesis.

I would like to thank all my collaborators without whom the project would not reach to this dimension. Their input with different experiments, theoretical calculations and useful discussion have increased my understanding and knowledge on this project a lot. In this context, I would like to specially thank Prof. Dr. Roser Valentí and Dr. Radu Coldea. I would also like to thank Dr. Stephan Rachel, Dr. Vladislav Kataev, Dr. Panchanan Khuntia and Dr. Yogesh Singh for privately sharing their comments and unpublished research outputs.

I would like to acknowledge all the supports I got from different members of the I. Physikalisches Institut. I am thankful to the all members of the low temperature physics and crystal growth group for their kind help and suggestions on many occasions, specially to Prof. Dr. K. Winzer for his many technical helps. I would also like to thank some of the past group members, Dr. Yogesh Singh for helping initially design this project, Dr. H. S. Jeevan for his great help, support and encouragement in my whole Ph.D. thesis work, Dr. Yoshi Tokiwa and Dr. Christian Stingl for their suggestions and help in many experiments. I would like to convey my special thanks and best wishes to some of the present doctoral students in our group, Melanie Schneider, Maik Schubert, Friedrich Freund and Jannis Maiwald. I thank my office mate Florian Fischgrabe for all the nice discussions we had in between work.

I am very much thankful to my great friends in Göttingen ("Crazy Göttingeners"). This Ph.D. thesis work would not be easy without their cheerful support in all the difficult times. I am grateful to all of them and specially thankful to the lunch group for each one refreshing hour in the stressful working days. I would like to thank all my friends who have supported 
and encouraged me to reach this level and specially like to thank all my IITR friends for encouraging me with their old but gold friendship.

At the end I would like to thank those few persons without whom I would not successful to fight this battle. I am grateful to my parents and my sister for their emotional support in all these years of study, far away from home and to my loving fiancée Moumita Nandi who has made the emotional and professional journey of my life smooth, cheerful and filled with feeling of completeness. I feel incredibly lucky to have you all in my life. 


\section{Contents}

Acknowledgements

1 Introduction $\quad 1$

2 Theoretical Background 3

2.1 Basics of crystalline solid state physics . . . . . . . . . . . . . . . 3

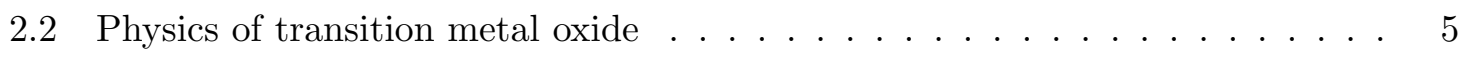

2.2 .1 Crystal Field . . . . . . . . . . . . . . . . 5

2.2 .2 Spin-orbit coupling . . . . . . . . . . . . . . . . . . 7

2.2 .3 Coulomb interaction . . . . . . . . . . . . . . . . 7

2.2 .4 Hund's Coupling . . . . . . . . . . . . . . . . . . 8

2.2.5 Comparison of different energy scales in TMOs . . . . . . . . . . 8

2.3 Physics under strong SOC limit . . . . . . . . . . . . . . . . . . . . . . . 9

2.3.1 $J_{\text {eff }}=1 / 2$ Kramers doublet in Iridates . . . . . . . . . . . . . . 9

2.3.2 Superexchange between $J_{\text {eff }}=1 / 2$ Kramers doublet . . . . . . . . 11

2.4 Physics of Honeycomb lattice SO material _ . . . . . . . . . . . . 13

2.5 Spin models for Honeycomb lattice iridate and rhodate . . . . . . . . . . . 14

2.5.1 Heisenberg-Kitaev $(\mathrm{HK})$ model . . . . . . . . . . . . . . . . . . . 14

$2.5 .2 J_{1}-J_{2}-J_{3}$ model . . . . . . . . . . . . . . . . . . 16

2.5.3 $J_{2}-J_{3}$ Heisenberg-Kitaev model . . . . . . . . . . . . . . 17

2.5.4 $2^{\text {nd }}$ neighbor Heisenberg-Kitaev model . . . . . . . . . . . . . 18

2.6 Itinerant picture of Honeycomb lattice iridates and rhodates . . . . . . . . . . 19

3 Experimental Techniques $\quad 21$

3.1 Sample Preparation . . . . . . . . . . . . . . . . . . . . 21

3.1.1 Polycrystalline material . . . . . . . . . . . . . . . 21

3.1.2 Sample preparation in special environment . . . . . . . . . . . . 22

3.1 .3 Single crystal growth . . . . . . . . . . . . . . . . . . . . . . . . . . . 23

3.1.3.1 Solid growth . . . . . . . . . . . . . . . 24

3.1.3.2 Liquid growth . . . . . . . . . . . . . . . . . . . . . . . . . . . . . . . .

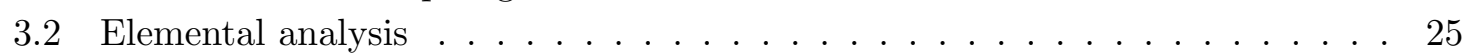

3.2.1 Energy-dispersive X-ray . . . . . . . . . . . . . . 25 
3.2.2 Inductively coupled plasma mass spectrometry (ICPMS) . . . . . . . 25

3.3 Structural characterization: X-ray diffraction . . . . . . . . . . . . 26

3.4 Magnetization Measurement . . . . . . . . . . . . . . . 26

3.5 Heat Capacity . . . . . . . . . . . . . . . . . . . . . . . . . . . . . . . . . . . . . .

3.6 Resistivity . . . . . . . . . . . . . . . . . 28

4 Honeycomb lattice Iridate $\mathrm{A}_{2} \mathrm{IrO}_{3}(\mathrm{~A}=\mathrm{Na}$ and $\mathrm{Li}) \quad \mathbf{2 9}$

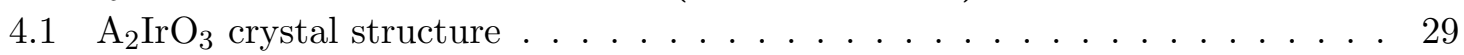

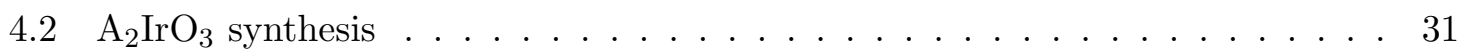

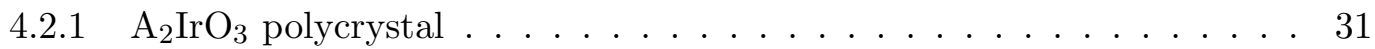

$4.2 .2 \mathrm{Na}_{2} \mathrm{IrO}_{3}$ single crystal growth . . . . . . . . . . . . . . . 33

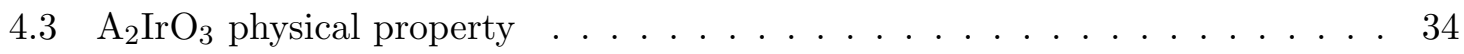

4.3.1 Structural characterization . . . . . . . . . . . . . 34

4.3.1.1 X-Ray Diffraction of $\mathrm{Na}_{2} \mathrm{IrO}_{3}$ single crystals . . . . . . . . 34

4.3.1.2 X-Ray Diffraction of $\mathrm{Li}_{2} \mathrm{IrO}_{3}$ polycrystal . . . . . . . . . 36

4.3.1.3 Pair Distribution Function (PDF) Analysis of $\mathrm{A}_{2} \mathrm{IrO}_{3} \quad \ldots \quad \ldots 38$

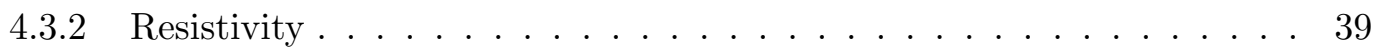

4.3 .3 Magnetization . . . . . . . . . . . . . . . . . . . . . . . . 42

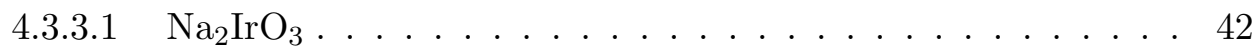

4.3.3.2 $\mathrm{Li}_{2} \mathrm{IrO}_{3} \ldots \ldots \ldots \ldots \ldots \ldots \ldots$

4.3 .4 Heat capacity . . . . . . . . . . . . . . . . . . 47

4.4 Disorder effect in $\mathrm{A}_{2} \mathrm{IrO}_{3} \ldots \ldots \ldots \ldots \ldots \ldots \ldots$

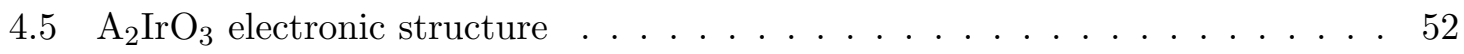

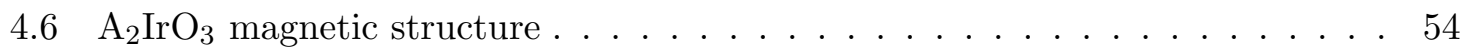

$4.6 .1 \quad \mathrm{Na}_{2} \mathrm{IrO}_{3} \ldots \ldots \ldots \ldots \ldots \ldots \ldots \ldots \ldots$

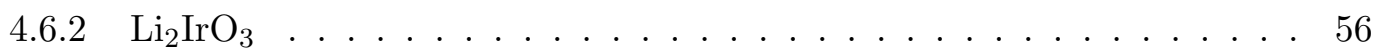

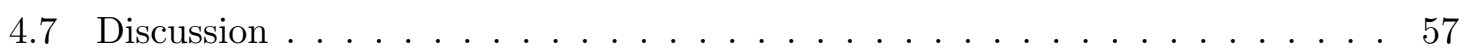

5 Doping effect on $\mathrm{A}_{2} \mathrm{IrO}_{3} \quad \mathbf{5 9}$

5.1 Isoelectronic doping at A site: $\left(\mathrm{Na}_{1-x} \mathrm{Li}_{x}\right)_{2} \mathrm{IrO}_{3} \ldots \ldots \ldots \ldots \ldots$

5.1 .1 Experimental details . . . . . . . . . . . . . . . 60

5.1 .2 Theoretical calculations . . . . . . . . . . . . . . . . 6 6 . . . . . . 61

5.1 .3 Results and Discussion . . . . . . . . . . . . . . . . 62

5.1.3.1 Low doping $(x<0.25) \ldots \ldots \ldots \ldots . \ldots \ldots 6$

5.1 .3 .2 Higher doping $(x>0.25) \ldots \ldots \ldots \ldots 65$

5.1 .4 Conclusions . . . . . . . . . . . . . . . 68

5.1.5 Preliminary study of electronic property on $\left(\mathrm{Na}_{1-x} \mathrm{Li}_{x}\right)_{2} \mathrm{IrO}_{3}$ single

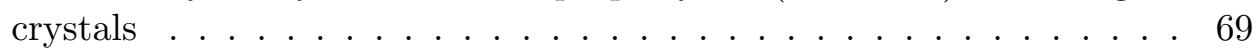

5.2 Magnetic impurity doping at Ir-site . . . . . . . . . . . . . . . . . . . 71

5.3 Nonmagnetic dilution of Ir-magnetism: $\mathrm{A}_{2}\left(\operatorname{Ir}_{1-x} \mathrm{Ti}_{x}\right) \mathrm{O}_{3} \ldots \ldots \ldots \ldots$

5.3 .1 Theoretical Prediction . . . . . . . . . . . . . . . . 74

5.3 .2 Experimental details . . . . . . . . . . . . . . . . . . . . 75

5.3.3 Results and Discussions . . . . . . . . . . . . . . 76

5.3.3.1 $\mathrm{Na}_{2}\left(\mathrm{Ir}_{1-x} \mathrm{Ti}_{x}\right) \mathrm{O}_{3}$ Results . . . . . . . . . . . . . . . 76

5.3.3.2 $\mathrm{Li}_{2}\left(\mathrm{Ir}_{1-x} \mathrm{Ti}_{x}\right) \mathrm{O}_{3}$ Results . . . . . . . . . . . . . . 79 
$5.3 .3 .3 \quad$ Discussions . . . . . . . . . . . . . . . . . . 85

6 Discussion of the $\mathrm{A}_{2} \mathrm{IrO}_{3}$ results on the context of Heisenberg-Kitaev $\begin{array}{lr}\text { Physics } & \mathbf{8 9}\end{array}$

$6.1 \mathrm{Na}_{2} \mathrm{IrO}_{3} \ldots \ldots \ldots \ldots \ldots \ldots \ldots \ldots$

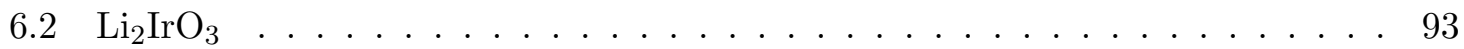

7 Honeycomb lattice Rhodate: $\mathrm{Li}_{2} \mathrm{RhO}_{3} \quad 97$

7.1 Sample synthesis . . . . . . . . . . . . . . . . . . . . . 98

7.2 Structural characterization . . . . . . . . . . . . . . . . . . 98

7.2 .1 Disorder in $\mathrm{Li}_{2} \mathrm{RhO}_{3} \ldots \ldots \ldots \ldots$

7.3 Electrical resistivity . . . . . . . . . . . . . . . . . . . 101

7.4 Magnetic property . . . . . . . . . . . . . . . . 103

7.5 Heat capacity . . . . . . . . . . . . . . . . . . . . . 105

7.6 Nuclear Magnetic Resonance(NMR) . . . . . . . . . . . . . . . . . 107

7.7 Band structure calculation . . . . . . . . . . . . . . . . . 108

7.8 Discussion . . . . . . . . . . . . . . . . . . . 111

8 Summary and Outlook 113

$\begin{array}{ll}\text { A Preliminary result of } \beta-\mathrm{Li}_{2} \mathrm{IrO}_{3} & 117\end{array}$

$\begin{array}{ll}\text { B Structural analysis of Li-Rh-O disordered phases } & 121\end{array}$

$\begin{array}{ll}\text { Bibliography } & 123\end{array}$ 

Dedicated to my parents and my fiancée Moumita Nandi... 



\section{Chapter 1}

\section{Introduction}

In condensed matter physics transition metal oxides (TMO) have provided a rich variety of physical phenomena by the mutual interplay of Coulomb correlation $(U)$, bandwidth (W) and spin-orbit coupling $(\lambda)$. Among them, the antiferromagnetic Mott ground state in layered $\mathrm{La}_{2} \mathrm{CuO}_{4}$ mediated by strong $U$ and the related high-temperature superconductivity in the charge-carrier doped materials; [1] metal-insulator transition, charge/orbital ordering in the rich phase diagram of manganites, [2] heavy fermion like large quasiparticle specific heat coefficient ( $\gamma$ value) in $\mathrm{LiV}_{2} \mathrm{O}_{4}$ [3] are some classic exotic states observed in 3d-TMOs. Superconductivity in $\mathrm{Sr}_{2} \mathrm{RuO}_{4}$ by spin-triplet pairing [4] and metamagnetic transition, electronic nematic phase, quantum criticallity in $\mathrm{Sr}_{3} \mathrm{Ru}_{2} \mathrm{O}_{7}[5,6]$ are some remarkable phenomena observed in $4 d$-TMOs.

In $5 d$ with all other energy scales, spin-orbit coupling (SOC) becomes highly relevant and orbital degeneracy is lifted by strong SOC. A recent path-breaking research has discovered the quantum spin Hall (QSH) effect in the time-reversal symmetric insulator or topological band insulator (TBI) having strong spin-orbit coupling which are characterized by invariance of $Z_{2}$ topological number. Strong SOC leads to a band inversion in QSH material which leads to conducting edge states in a bulk insulator. TBI was theoretically predicted in Kane-Mele model and experimentally realized in spin-orbit semiconductors. [7, 8] Recently Pesin et. al. theoretically a predicted topological Mott insulator (TMI) state in Ir-based materials by the effect of strong spin-orbit interaction and Coulomb correlation. [9] Also recently, a novel spin-orbit Mott insulating state is realized in $\mathrm{Sr}_{2} \mathrm{IrO}_{4}$. [10]

$5 d$-TMO posses a huge possibilities for rich physical phenomena by the effect of strong SOC. Honeycomb lattice iridates $\mathrm{Na}_{2} \mathrm{IrO}_{3}$ and $\mathrm{Li}_{2} \mathrm{IrO}_{3}$ become interest of study due to its relevance in honeycomb lattice Kitaev physics. The model given by A. Kitaev, namely 
Kitaev model describes the highly anisotropic exchange of $\mathrm{SU}(2)$ spin- $1 / 2$ moments on the honeycomb lattice, called Kitaev exchange. It has a a gapless spin liquid (Kitaev spin liquid) ground state with emergent Majorana fermion excitations.[11] In the presence of a magnetic field a gapped $Z_{2}$ spin liquid, and a topologically ordered phase with non-Abelian quasiparticle statistics is proposed in Kitaev model.[12] Chaloupka et. al. described a Heisenberg-Kitaev model for the honeycomb lattice material $\mathrm{A}_{2} \mathrm{IrO}_{3}(\mathrm{~A}=\mathrm{Na}, \mathrm{Li})$ having three magnetic ground states : Néel AF, stripy AF and Kitaev spin liquid. $\mathrm{Na}_{2} \mathrm{IrO}_{3}$ was proposed to be a Kitaev spin-liquid.[13] At the similar time, from a tight-binding model analysis together with the first principles band structure calculation $\mathrm{Na}_{2} \mathrm{IrO}_{3}$ was predicted to be a topologically non trivial QSH insulator by Shitade et. al.[14]

The first detailed experimental study on $\mathrm{Na}_{2} \mathrm{IrO}_{3}$ single crystals by Yogesh Singh et. al. clearly showed that it is a long range antiferromagnetically ordered insulator, not a Kitaev spin-liquid.[12] This started a new era in iridate community to experimentally understand the honeycomb lattice iridates $\mathrm{Na}_{2} \mathrm{IrO}_{3}$ and $\mathrm{Li}_{2} \mathrm{IrO}_{3}$ which are closest candidates for exotic HK physics. 


\section{Chapter 2}

\section{Theoretical Background}

\subsection{Basics of crystalline solid state physics}

One of the earliest ways to classify solid state of a matter was "Metal" and "Insulator", based upon conduction of electron. First rigorous theory was derived by Paul Drude to explain metals: "free electron theory", based on the classical statistics. This theory successfully explained electrical properties of many metals but partially failed to explain many thermodynamic properties. Sommerfeld re-derived "free electron theory" considering bound state of electron due to Pauli exclusion principle. Metals were assumed as non interacting Fermi gas which successfully explained many thermodynamic properties of metals where Drude's models failed. But what about insulators!! Free electron approximation failed there because both of the model assumed that positive ions do not have any effect on motion of electrons. To consider the effect of ions, first observational fact came out from x-ray diffraction experiment that atoms or these positively charged ions are not distributed randomly but arranged in a periodic array. The periodic lattice of ions coined new classification in solid state physics called crystalline and amorphous state material.[15]

Periodic arrangements of atoms in a crystalline material are classified into different Bravais lattice, defined by different kind of symmetries. Atom consists of a nuclei (ion) and electrons. So a crystalline material can be thought as electrons in a potential $U(\mathbf{r})$ due to periodic arrangement of the ions with the periodicity of the underlying Bravais lattice vector; i.e.,

$$
U(\mathbf{r}+\mathbf{R})=U(\mathbf{r})
$$


for all Bravais lattice vector $\mathbf{R}$. In addition, there could be another potential due to electronelectron interaction or electron correlation. In case of independent electron approximation, total potential is represented by an effective one-electron potential $U(\mathbf{r})$ at each site of the crystal excluding this electron-electron correlation potential. To determine electron motion now one has to solve the Schrödinger equation with potential $U(\mathbf{r})$. Felix Bloch gave a pioneering theorem for such a periodic system, which says that potential should have a periodicity of lattice and wave function is given by

$$
\begin{aligned}
\psi_{n \mathbf{k}}(\mathbf{r}) & =e^{i \mathbf{k} \cdot \mathbf{r}} u_{n \mathbf{k}}(\mathbf{r}) \\
u_{n \mathbf{k}}(\mathbf{r}+\mathbf{R}) & =u_{n \mathbf{k}}(\mathbf{r})
\end{aligned}
$$

Now considering a weak periodic potential and Bloch's wave function (Eqn. 2.2) lot of pure metals were well described. Another popular approach to explain different insulators and metals was tight-binding model where solid is treated as weakly interacting neutral atoms. Wave function was calculated from the overlap of atomic orbitals of nearest neighbors, called localized atomic wave function. There were more developments to understand the band structure with independent electron approximation which was described by different models.[15] But to make a general understanding of solid material, one has to understand the periodic potential.

First such approach was Hartree equation and Hatree-Fock equation. Here, electronelectron interaction was taken into account in the form of an average self-consistent field which is interaction of a electron in one site with the average field of all other electrons. Later deviation from the experimental results proved that the Hatree-Fock approach was not the right way to explain electron-electron interaction. Though it was first phenomenal approach to treat many body interacting problem, it resulted in also some great understanding like exchange, screening etc. Theories derived from independent electron approximation Thomas-Fermi model and landmark theory of Landau Fermi liquid theory and Hartree-Fock theory gave good explanation of first few members of periodic table.

But main problem was still present there to explain the periodic potential including electron-electron correlation in proper way. But it became necessary to find a proper theory when band theory of solid, based upon independent electron approximation failed to explain band structure of some significant materials consisting transition metal e.g. $\mathrm{CoO}, \mathrm{FeO}, \mathrm{NiO}$ etc. In case of $\mathrm{CoO}$, band theory predicted it as a metal because Co has odd numbers of electron in the valence band but insulating behavior was found experimentally. Insufficiency 
of the band theory was pointed out to explain transition metal systems where many equivalent energy scales start to play important roles together including electron correlation. But solution of this problem took decade until Gutzwiller, Hubbard, Kanamori came up separately with a new method to treat electron-electron interaction in 1963, which gave birth of Hubbard model and started a new era of correlated electron physics.[16]

\subsection{Physics of transition metal oxide}

In Transition metal compounds (oxides) (TMO), the transition metal (TM) atom is surrounded by ligand atoms $\left(\mathrm{O}^{2-}\right)$ to help in the formation of a solid by the increase in cohesive energy via covalent bonds of the TM and O. Transition metal atom has outermost filled or partially filled d-orbitals, hence they are called d-electron system. Total anagular momentum $(L)$ of the $d$-orbitals is 2 with five fold orbital degeneracy : $L_{z}=2,1,0,-1,-2$. Five d-orbitals are $d_{3 z^{2}-r^{2}}, d_{x^{2}-y^{2}}, d_{x y}, d_{y z}, d_{z x}$. When a solid is formed with the TM ion the atomic orbitals create bands due to the periodic potential of atoms. The bandwidth is basically determined from the overlap of two $d$ orbitals of two adjacent transition metals. Due to very less spatial extension of $d$-orbitals TM-bands are narrower than alkaline metals.[17]

In TMOs, the overlap is often determined by indirect transfer between $d$-orbitals through ligand $\mathrm{O}-p$ orbitals. The bandwidth is determined by the hybridization of the $d$ wave function of a TM atom and the $p$ wave function of the adjacent ligand atom surrounding it. Because of this indirect transfer through ligand atomic orbitals, in general the TMO bandwidth becomes narrower.

\subsubsection{Crystal Field}

The hybridization between a TM and a O-orbital has another effect: it lifts the five-fold degeneracy of $d$-orbital band. TM ion, surrounded by ligand oxygen ions $\left(\mathrm{O}^{2-}\right)$ gives rise to the crystal field potential which quenches the orbital angular momentum by introducing the crystal field (CF) splitting of the d orbitals.[18, 19] Now depending upon geometry of ligands, CF splittings of the five $d$-bands are different. Possible different geometries are "Octahedral", "Square-planer", "Tetrahedral" etc. But most common form of the ligand geometry in TMOs is octahedral where TM ion is surrounded by six equidistant $\mathrm{O}^{2-}$ ion. The ligand $\mathrm{O}$ atoms have negative valence so the crystal field of electrons in the direction of the ligand atom is higher than in other directions due to electrostatic repulsion. Hence under CF $d_{3 z^{2}-r^{2}}$ and $d_{x^{2}-y^{2}}$ orbitals which point towards $z$ and $x, y$-axis respectively shift 
to higher energy by forming $e_{g}$ energy state and $d_{x y}, d_{y z}, d_{z x}$ orbitals shift to lower energy by forming $t_{2 g}$ energy state (Fig. $2.1 \mathrm{~b}$ ). Energy difference between the two states is $10 D q$ and for typical TMOs which is $2-3 \mathrm{eV}$.

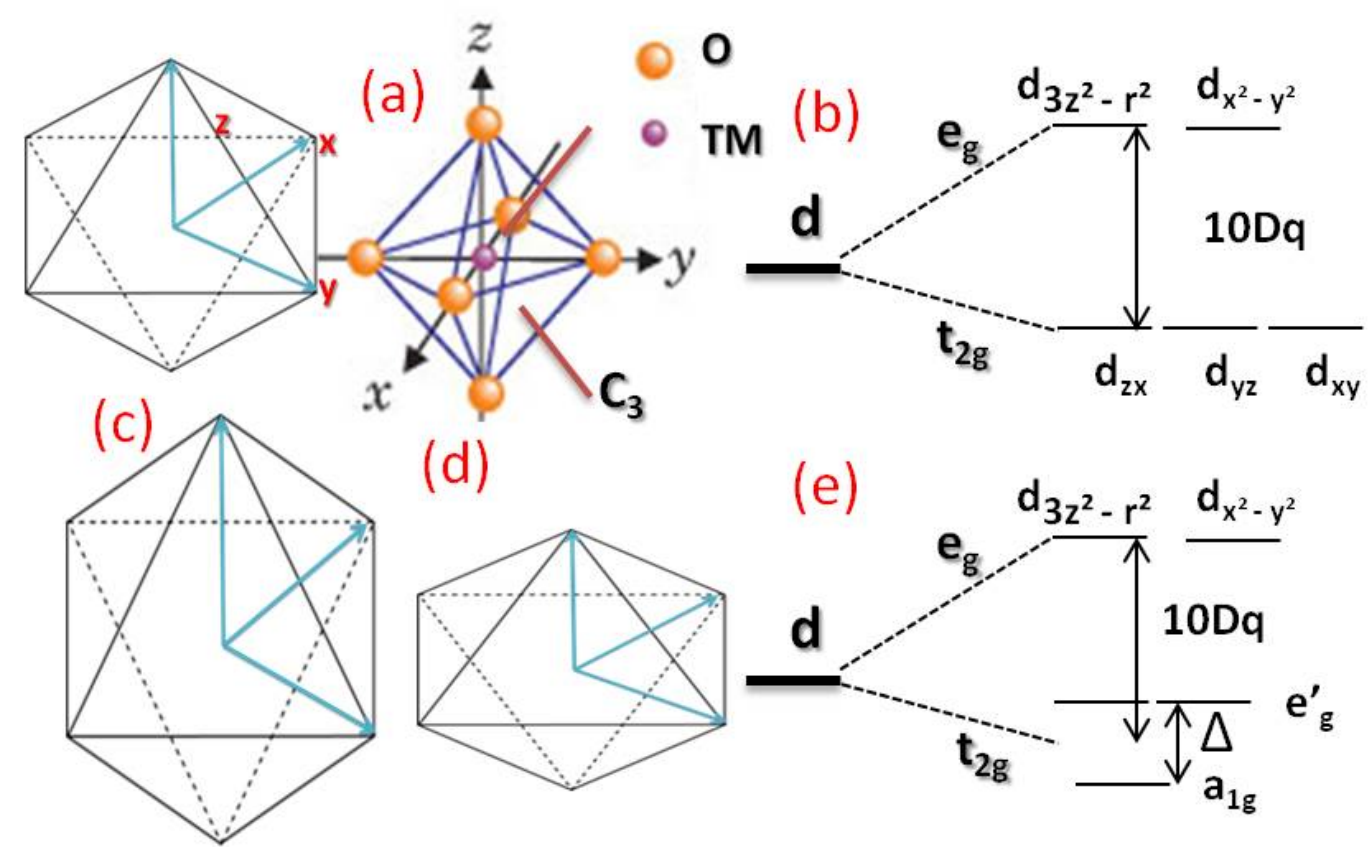

Figure 2.1: (a) Undistorted octahedron and TM ion in octahedral ligand environment shown in a cubic coordinate system. $C_{3}$ is cubic 3 -fold rotation axis. (b) Splitting of $d$-orbital by octahedral crystal field $(\mathrm{CF})$. (c) Elongated (d) compressed octahedron along trigonal direction $\left(C_{3}\right.$-axis) (e) Change the octahedral $\mathrm{CF}$ splitting due to trigonal distortion.

In the octahedral CF splitting, all the TM-O distances are equal. But due to different causes, (TM) $\mathrm{O}_{6}$ can distort. Due to certain electronic configuration if the octahedron distorts along any cubic axis $(x, y, z)$ by elongating or contracting then it is called tetragonal distortion as the octahedron is distorted along 4-fold $\mathrm{C}_{4}$ axis.[20] One of such example is John-Teller distortion commonly found in $3 d$ TMOs like Manganites and Cuprates. Due to change in TM-TM bonding, a (TM) $\mathrm{O}_{6}$ can elongate or compress along $C_{3}$-axis which is called trigonal distortion (Fig. $2.1 \mathrm{c}, \mathrm{d}$ ). This $C_{3}$-axis is basically (111) cubic axis, shown in Fig. 2.1 a. Due to such a small distortion the $t_{2 g}$ state splits into $e_{g}^{\prime}$ and $a_{1 g}$ states by $\Delta$ energy difference. Usually $\Delta<<10 D q$, hence no mixing between $t_{2 g}$ and $e_{g}$ happens in trigonal distortion which very commonly happens in case of tetragonal distortion.[20, 21] 


\subsubsection{Spin-orbit coupling}

Interaction between orbital angular momentum $(\mathbf{L})$ and spin angular momentum $(\mathbf{S})$ of a electron in a single site is a important factor in determining electronic state of a solid. This spin-orbit ( $\mathrm{SO}$ ) Hamiltonian is given by:

$$
H_{S O}=\lambda \mathbf{L} \cdot \mathbf{S}
$$

This Hamiltonian operating on one electron wave function $\psi:\left\langle\psi\left|H_{S O}\right| \psi\right\rangle$ gives eigenvalue $\lambda$ where $\lambda \propto Z^{4}, Z$ is atomic quantum number.

\subsubsection{Coulomb interaction}

Coulomb interaction represents electron-electron interaction at the same site and same orbital.[19] Two electrons in the same orbital will face some Coulomb repulsion which will restrict the hopping of electrons to the next site. So basically this interaction localizes the electron in a single site. This interaction actually turns some partially filled valence band metal into an insulator, restricting electron hopping. Hubbard model is the first model which explains the electron motion in a lattice considering electron correlation :

$$
H=-t \sum_{<\mathbf{j}, \mathbf{l}>, \sigma}\left(c_{\mathbf{j} \sigma}^{\dagger} c_{\mathbf{l} \sigma}+c_{\mathbf{j} \sigma}^{\dagger} c_{\mathbf{l} \sigma}\right)+U \sum_{\mathbf{j}} \hat{n}_{\mathbf{j} \uparrow} \hat{n}_{\mathbf{j} \downarrow} .
$$

Where $\mathrm{c}^{\dagger} \mathbf{j} \sigma$ creates an electron in the Wannier state $\phi\left(\mathbf{r}-\mathbf{R}_{\mathbf{j}}\right)$ with spin $\sigma, \mathbf{j}$ being lattice site index. $\hat{n}_{\mathbf{j} \sigma}=\mathrm{c}_{\mathbf{j} \sigma}^{\dagger} c_{\mathbf{j} \sigma}$ is the corresponding occupation number operator. In the Eqn. 2.5 first term, summation over nearest neighbor pairs $\mathbf{j}, \mathbf{l}$ is kinetic energy term describing hopping of electrons where $t$ is the hopping matrix. Second term describes the Coulomb interaction of electrons at the same site or same orbital where $U$ is called Hubbard $U$ or Coulomb repulsion. Now if both $U$ and $t$ have finite value, in the $U<t$ limit free electron hopping will prefer a metallic state but in the $U>t$ limit electrons will be forced to localize in a single site and electric charge can not flow through lattice hence it will give rise to a insulating state. This is the first model to explain an insulating state of a material where tight-binding model failed.

Electron has another property other than charge which is spin. Pauli exclusion principle does not allow electron hopping between two sites having parallel spin configuration. Hence in a material with exactly half filled $d$-orbital where all the electrons in the same site are parallel by Hund's coupling, only way to allow hopping between neighboring site if their 
spins are oriented anti parallel to each other. This causes antiferromagnetism. Hubbard $U$ creates spin-exchange interaction (direct exchange or superexchange) between two electron spins at the neighboring site which results in magnetism.

\subsubsection{Hund's Coupling}

Hund's coupling represents electron-electron interaction at the same site but different orbital by Hund's rule.[19] One of the important consequence of the Hund's rule is that it favors parallel alignment of spins rather than two anti-parallel spins in the same orbital. It means Hund's coupling favors a high spin state. Hund's coupling energy is basically the energy difference between high spin state and low spin state i.e. energy saved by putting all the spins in different $m_{L}$ states in parallel.

\subsubsection{Comparison of different energy scales in TMOs}

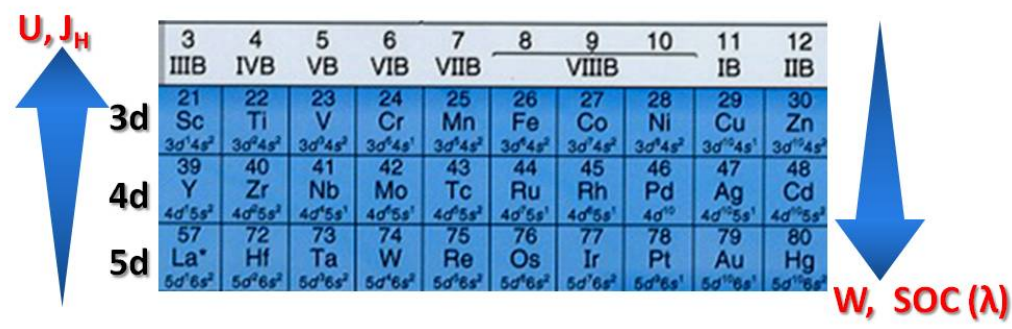

Figure 2.2: Variation of different energy scales Bandwidth $(W)$, Coulomb repulsion $(U)$, Hund's coupling $\left(J_{H}\right)$, Spin-orbit coupling $(\lambda)$ along $3 d$ to $5 d$ in the Periodic table.

In the TMOs, TM ion contributes either $3 d$ or $4 d$ or $5 d$ orbital. From $3 d$ to $5 d$ orbital, spatial extension of $d$-orbital increases, hence bandwidth (W) of the TMO-band formed by $d$-orbital increase if we go down in the periodic table (Fig. 2.2). This causes a strong increase in orbital angular momentum L. $H_{S O}$ can not be treated as perturbation, moreover $\lambda \propto Z^{4}$. So along a group in the periodic table $\lambda$ increases downwards. For $5 d$ elements $\lambda$ becomes an important energy scale, having value $0.4-0.5 \mathrm{eV}$, whereas it is only $0.05 \mathrm{eV}$ for $3 d$ elements. Moreover, it is found that in Iridium $\lambda$ is higher than its neighbors (e.g. Rhenium) in the same period.[22]

Now Coulomb repulsion will be large for smaller bandwidth $(\mathrm{W})$ because it is inversely proportional to electron-electron distance $U \propto e /\left|\mathbf{r}_{1}-\mathbf{r}_{\mathbf{2}}\right| . U$ reduces from $5-10 \mathrm{eV}$ in $3 d$ to $1-2 \mathrm{eV}$ in $5 d$. Hund's coupling $J_{H}$ has much higher value in $3 d$ compared to $5 d$ and it decreases with the increase of bandwidth. In $3 d$ materials like manganites it plays a crucial 
role to achieve high spin state, it is order of crystal field splitting of $e_{g}$ and $t_{2 g}$. $[23,24]$ In $5 d$-TMO all the energy scales $U, \lambda$ and $J_{H}$ become almost same order of magnitude. So, by competition or complement of all these energy scales, a very rich variety of physical phenomena is expected in $5 d$ materials. Most importantly, equivalence of Coulomb repulsion and SOC in $5 d$ is very unique compared to all other $d$-orbital elements in periodic table.

\subsection{Physics under strong SOC limit}

In iridates, under strong spin-orbit coupling (SOC) limit $H_{S O}$ can not be treated as perturbation, so that $\mathbf{S}$ and $\mathbf{L}$ do not commute with the total atomic Hamiltonian hence they are not good quantum numbers. Rather by the LS coupling total angular momentum $\mathbf{J}=\mathbf{L}$ $+\mathbf{S}$ is the good quantum number in the strong SOC limit. Total angular momentum can have values from $|\mathbf{L}+\mathbf{S}|$ to $|\mathbf{L}-\mathbf{S}|$.

\subsection{1 $J_{\text {eff }}=1 / 2$ Kramers doublet in Iridates}

Atomic configuration of Iridium : $[\mathrm{Xe}] 4 f^{14} 5 d^{7} 4 s^{2}$. In Iridates $\left(\mathrm{IrO}_{2}, \mathrm{~A}_{2} \mathrm{IrO}_{3}(\mathrm{~A}=\mathrm{Na}, \mathrm{Li})\right.$, $\mathrm{Sr}_{2} \mathrm{IrO}_{4}$ etc.) $\mathrm{Ir}^{4+}$ ion forms covalent bond with $\mathrm{O}^{2-}$ ion sharing 4 valence electron of Ir. 2 electrons come from $4 s$ orbital and another 2 from $5 d$ orbital. Hence in Iridates valence band has $5 d^{5}$ state, half filled $5 d$-orbital. Due to octahedral CF of oxygen ligands, $5 d$ states splits into $t_{2 g}$ and $e_{g}$ as explained in the previous section. Now if it has to follow Hund's rule, then for half filled state electrons should be in high spin state with 3 electrons in triply degenerate $t_{2 g}$ orbital and another 2 electrons in doubly degenerate $e_{g}$ orbital. But as we discussed, in $5 d$ Hund's coupling energy $J_{H}$ is small, of the order of $0.6 \mathrm{eV}$ which is much smaller than CF splitting $(10 D q=2-3 \mathrm{eV})$. Hence all the 5 electrons will be in lower $t_{2 g}$ state, having one hole (Fig. 2.3).

To determine the effect of SOC one has to define orbital angular momentum $|\mathbf{L}|$ of partially filled $t_{2 g}$ orbital. In the cubic crystal field the eigen functions of a d-electron are not spherical harmonics $\left(Y_{l}^{m}\right)$ but are given by the linear combination of it, for $t_{2 g}$ it is: [19] $|X Y\rangle \propto 1 / i \sqrt{2 \pi / 5}\left(Y_{2}^{2}-Y_{2}^{-2}\right)$

$|Y Z\rangle \propto \sqrt{2 \pi / 5}\left(Y_{2}^{-1}+Y_{2}^{1}\right)$ $|Z X\rangle \propto 1 / i \sqrt{2 \pi / 5}\left(Y_{2}^{-1}+Y_{2}^{1}\right)$

Angular momentum matrix element for all the 5 electrons in $t_{2 g}$ will be given by $\left\langle t_{2 g}|\mathbf{L}| t_{2 g}\right\rangle$. Resulting angular moment matrix element is same as for the $|p\rangle$ orbital. Hence 


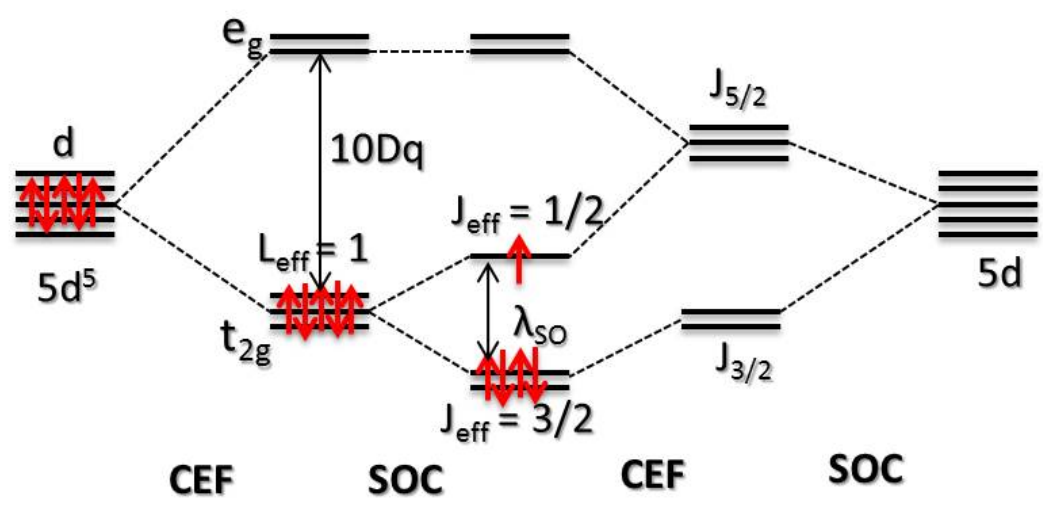

Figure 2.3: Splitting of $5 d^{5}$ state under octahedral crystal electric field (CEF) and spinorbit coupling (SOC). Schematics of electronic level splitting of $J_{\text {eff }}=1 / 2$ spin-orbit Mott insulating state. Red arrows represent 5 spins. Schematic drawing is based upon Ref. [10] by Kim et. al.

orbital angular moment of $5 d$-orbital $L=2$ is quenched to $L=1$ in iridates by crystal field. In $t_{2 g}, L_{e f f}=1$ and eigen states are given by $|1,1\rangle,|1,-1\rangle$ and $|1,0\rangle \cdot[25,26]$

Effective spin angular momentum is $S_{\text {eff }}=1 / 2$ having one hole $t_{2 g}$ in $5 d^{5}$ state. By LS coupling total angular momentum $|\mathbf{J}|$ ranges from $|\mathbf{L}+\mathbf{S}|$ to $|\mathbf{L}-\mathbf{S}|$. So the 3-fold degenerate $t_{2 g}$, with $L_{e f f}=1$ and $S_{\text {eff }}=1 / 2$ split into $J_{\text {eff }}=1 / 2$ state and doubly degenerate $J_{\text {eff }}=3 / 2$ state due to strong SOC. $J_{\text {eff }}=3 / 2$ state is branched from the $J_{5 / 2}$ manifold due to larger crystal field and $J_{\text {eff }}=1 / 2$ state from $J_{3 / 2}$ state hence former is energetically lower than the other (Fig. 2.3).[10] In iridates, among 5 electrons, 4 electrons are in the $J_{\text {eff }}=3 / 2$ orbital and one is in the $J_{1 / 2}$ orbital resulting in partially filled $J_{\text {eff }}=1 / 2$ state. These $J_{\text {eff } f}$ states are not pure orbital state, rather admixture of orbital and spin state and called isospin states. A complex wave function is given by: $\left|J_{e f f}=1 / 2, m_{J_{\text {eff }}}= \pm 1 / 2\right\rangle$ $=(|Y Z \pm \sigma\rangle \mp i|Z X \pm \sigma\rangle \mp i|X Y \pm \sigma\rangle) / \sqrt{3}$ where $\sigma$ is the spin state (1/2).[10] These $\left|J_{e f f}, m_{J_{e f f}}\right\rangle$ states are called isospins and they are more symmetric than pure orbital states. In the solid, bands created by overlapping $J_{\text {eff }}$ states are much narrower. Physics with strong SOC coupling will be much different from physics in weak SOC limit.

Lets investigate empirically, the difference in the electronic property between the two scenarios. A transition metal oxide with partially filled $t_{2 g}$ orbital is expected to be a metal from band theory as a non interacting system. And if Coulomb repulsion is much smaller than one-electron hopping $(U<<t)$ then it will result in a $t_{2 g}$ paramagnetic band metal (Fig. 2.4a). But in case of $U>t, U$ is greater than the band width $W=2 z t$. So the atomicband is split into ground state and and first excited state namely, lower Hubbard band 


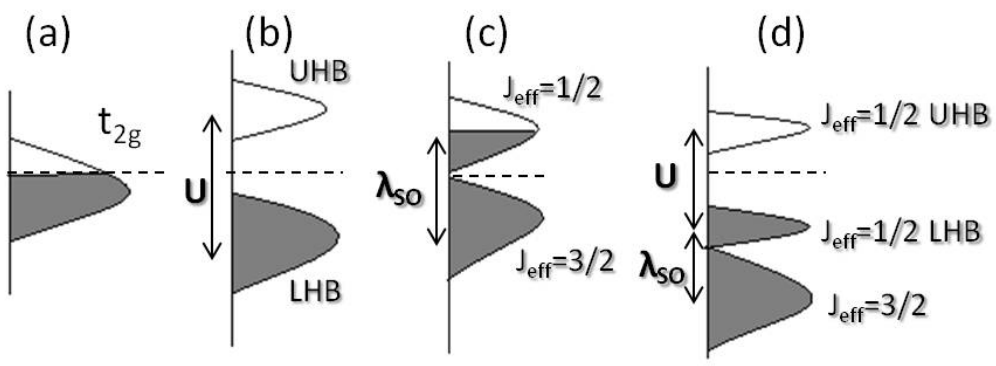

Figure 2.4: (a) Partially filled $t_{2 g}$ orbital, splitting of it: (b) by strong Coulomb repulsion $U$ into upper Hubbard band (UHB) and lower Hubbard Band (LHB) (c) by strong spinorbit coupling (SOC) into partially filled $J_{\text {eff }}=1 / 2$ and filled $J_{\text {eff }}=3 / 2$ state with SOC splitting $\lambda_{S O}$. (d) Further splitting of partially filled $J_{\text {eff }}=1 / 2$ band into filled LHB and empty UHB. Last two band splitting corresponds to electronic state in Fig. 2.3. Schematic drawing is based upon Ref. [10] by Kim et. al.

(LHB) and upper Hubbard band (UHB) and they are separated by $U$. For partially filled $d$-orbital LHB will be filled and UHB is empty resulting a Mott insulating state (Fig. 2.4b).

Now if a partially filled band is relativistic $J_{\text {eff }}$ band then SOC splits the $t_{2 g}$ band into partially filled $J_{e f f}=1 / 2$ band and $J_{e f f}=3 / 2$ band which are separated by $\lambda_{S O}$, spin orbit coupling energy (Fig. 2.4c). Now a very small Coulomb interaction can split the partially filled narrow $J_{\text {eff }}=1 / 2$ band into $J_{\text {eff }}=1 / 2 \mathrm{LHB}$ and $J_{\text {eff }}=1 / 2$ UHB (Fig. $2.4 \mathrm{~d}$ ). For $5 d^{5}$ state depicted in Fig. 2.3 upper most band is totally empty and next one is totally filled and separated by $U$. This gives rise to a new Mott insulating state mediated by SOC and such material is called $J_{\text {eff }}=1 / 2$ spin-orbit Mott insulator. Electron in the spatially extended $5 d$ orbital has very small $U$ which can not split $t_{2 g}$ alone but it is much easier to split narrower $J_{\text {eff }}=1 / 2$ band by small $U$. Hence this insulating state is mainly due to strong SOC. First reported such state is $\mathrm{Sr}_{2} \mathrm{IrO}_{4}$.[10] In this thesis this scenario will be investigated in honeycomb lattice iridates and rhodates.

\subsubsection{Superexchange between $J_{\text {eff }}=1 / 2$ Kramers doublet}

In the TMOs transition metal (TM) isospins are present usually in oxygen environment. So in the strong SOC limit, exchange interaction between two isospins will have dominating superexchange through oxygen.[27] Now in the oxygen octahedral environment two superexchange geometry is possible, (i) $180^{\circ}$ TM-O-TM bond in Fig. 2.5(a) or (ii) TM-O-TM $90^{\circ}$ bond, shown in Fig. 2.5(b). G. Jackeli et. al. derived superexchange Hamiltonian for both of this geometry.[27] 


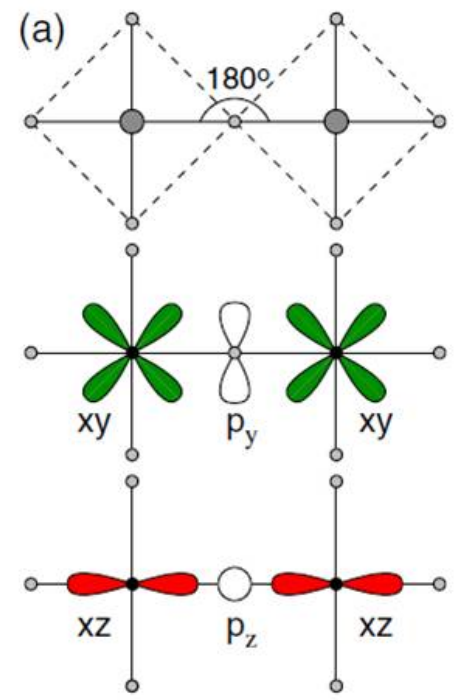

(b)
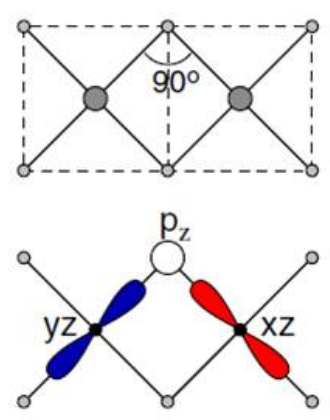

Figure 2.5: Two possible geometries of a TM-O-TM bond. The large (small) dots stand for the transition metal (oxygen) ions. (a) A $180^{\circ}$-bond formed by corner-shared octahedra, and (b) a $90^{\circ}$-bond formed by edge-shared octahedra. Reprinted from [27]

(i) $180^{\circ}$ bond : Two cornered shared octahedra form this TM-O-TM geometry. Nearest neighbor $t_{2 g}$ hopping matrix is diagonal in the orbital space. For a hopping along the bond in $x$-direction $|x y\rangle$ and $|x z\rangle$ orbitals are active and hopping between two such same orbitals happen through oxygen $p_{y}$ and $p_{z}$ orbitals respectively. Exchange Hamiltonian, determined including all these hopping has an isotropic Heisenberg term and a small anisotropic pseudodipolar interaction term(Eqn. 2.6).[27]

$$
H_{i j}=J_{1} \mathbf{S}_{i} \cdot \mathbf{S}_{j}+J_{2}\left(\mathbf{S}_{i} \cdot \mathbf{r}_{i j}\right)\left(\mathbf{r}_{i j} \cdot \mathbf{S}_{j}\right)
$$

In $\mathrm{Sr}_{2} \mathrm{IrO}_{4}$, corner-shared $\mathrm{IrO}_{6}$ octahedra forms this TM-O-TM near $180^{\circ}$ bond angle which is deviated due to rotation of the octahedra along bond direction and trigonal distortion of the octahedra.[28]

(ii) $90^{\circ}$ bond : Two edge sharing octahedra form $90^{\circ}$ TM-O-TM bond. Here $|x z\rangle$ and $|y z\rangle$ orbitals are active in hopping along a bond in the $x y$ plane of the octahedral system. However, the hopping matrix has now only nondiagonal elements and there are two possible paths for a charge transfer [via upper and lower oxygen, see Fig. 2.5(b)]. Wave function of the isospin orbitals are complex in strong SOC limit, so, two transfer amplitudes via upper and lower oxygen interfere in a destructive manner in this geometry and the isotropic part of the Hamiltonian vanishes and only the finite anisotropic part remains. This anisotropic part is different for hopping perpendicular to $x, y$ and $z$-axis. Exchange interaction depends on the spatial orientation of a given bond i.e. anisotropic. The exchange Hamiltonian can be written as,

$$
H_{i j}^{\gamma}=-J S_{i}^{\gamma} S_{j}^{\gamma}
$$




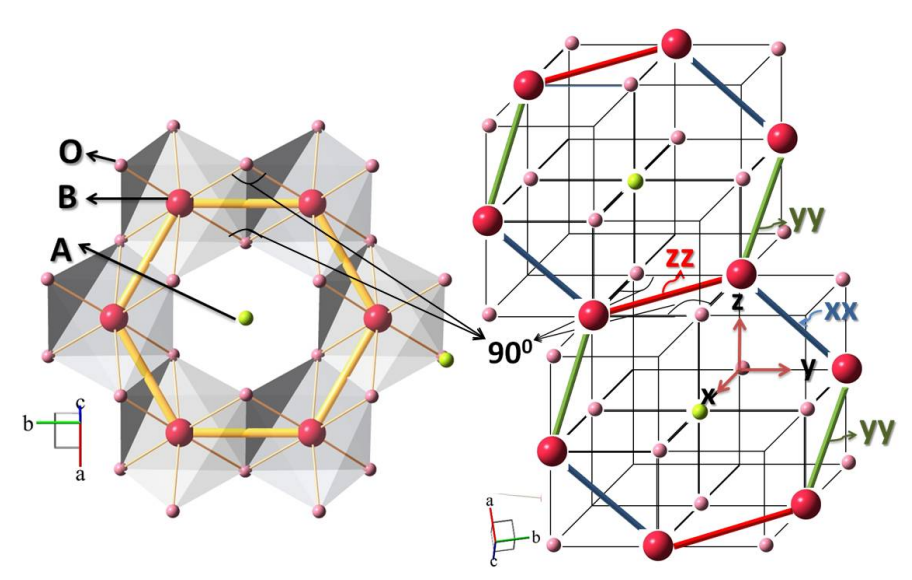

Figure 2.6: (Left) Honeycomb lattice formed by $\mathrm{A}_{2} \mathrm{BO}_{3}$ structure, $\mathrm{A}=$ nonmagnetic ion, $\mathrm{B}=\mathrm{TM}$, magnetic ion. $\mathrm{O}=$ Oxygen. (Right) $\mathrm{A}_{2} \mathrm{BO}_{3}$ in the cubic coordinate (xyz) of $\mathrm{BO}_{6}$ octahedra. Edge sharing octagedra forms $90^{\circ} \mathrm{B}-\mathrm{O}-\mathrm{B}$ bond and hence $S_{i}^{\gamma} S_{j}^{\gamma}$ Kitaev interaction appears on a $\gamma \gamma$ bond perpendicular to octahedral $\gamma$-axis, $\gamma=x, y, z$ axis.

A $i j$ bond is labeled as $\gamma$ - bond lying in a plane perpendicular to $\gamma(=x, y, z)$ axis.

This exchange interaction $J$ represents highly bond dependent and totally anisotropic interaction. $J$ is not Heisenberg type rather Ising type with preferred directions. This Hamiltonian in Eqn. 2.7 is quantum analog of the so-called compass - model. A. Kitaev derived same Hamiltonian for $S=1 / 2$ on a honeycomb lattice which shows novel anyonic excitations with exotic fractional statistics and topological degeneracy. This model has a spin-liquid state called Kitaev spin-liquid mediated by majorona fermions. Kitaev model has fundamental importance in quantum computation.[11] In last few years this exactly solvable model attracted many theoretical studies in search of gapless and gapped excitations and topological phases under certain perturbation.[12, 14, 29, 30, 31, 32]

\subsection{Physics of Honeycomb lattice SO material}

In layered $\mathrm{A}_{2} \mathrm{BO}_{3}$ structure, edge sharing $\mathrm{BO}_{6}$ octahedra form the honeycomb lattice having A-ion at the center shown in Fig. 2.6 ( $a, b, c$ are the axes of the unit cell of the structure). In this figure, right panel shows $\mathrm{A}_{2} \mathrm{BO}_{3}$ in the cubic coordinate of $\mathrm{BO}_{6}$ octahedra. $90^{\circ} \mathrm{B}-\mathrm{O}-\mathrm{B}$ (TM-O-TM) bonding geometry is realized here. There are three non-equivalent B-B bonds $x x, y y$ and $z z$ perpendicular to one of the cubic axes $x, y$ and $z$ respectively. The bonds are different due to different TM-O-TM hopping $(\mathrm{B}=\mathrm{TM})$. Along $x x$ bond hoping happens between two neighboring TM (B) orbital $|x y\rangle$ and $|x z\rangle$ via oxygen $p_{x}$ orbital resulting $S_{i}^{x} S_{j}^{x}$ Kitaev spin-coupling, similarly for $S_{i}^{y} S_{j}^{y}$ coupling, hopping happens between $|x y\rangle$ and $|y z\rangle$ orbital via $\mathrm{O} p_{y}$ orbital and for $S_{i}^{z} S_{j}^{z}$, between $|x z\rangle$ and $|y z\rangle$ orbital via $p_{z}$ orbital. Each $\mathrm{BO}_{6}$ octahedron is connected to three neighboring octahedra (Fig. 2.6) so that three $t_{2 g}$ orbitals of that B-ion participate in three different hopping with three neighboring B-ion. Each B 
(TM) atom is connected via three different $\gamma \gamma$ bond with neighboring B atom resulting three different $S_{i}^{\gamma} S_{j}^{\gamma}$ Kitaev exchange branching from each B-atom.

In honeycomb lattice spin orbit material $\mathrm{Na}_{2} \mathrm{IrO}_{3}, \mathrm{Li}_{2} \mathrm{IrO}_{3}$ and $\mathrm{Li}_{2} \mathrm{RhO}_{3}$, Ir having strong SO coupling $\lambda \approx 0.4 \mathrm{eV}$ and Rh having $\lambda \approx 0.15 \mathrm{eV}$ create honeycomb lattice of SO Krammers doublet. This is the ideal scenario for Kitaev exchange as described above. Hence one would expect low-energy Kitaev excitations in these systems which is investigated in this thesis in great detail.

\subsection{Spin models for Honeycomb lattice iridate and rhodate}

But in case of the real materials, truth may be much more complicated. Other exchange interactions can be present between B-ions other than Kitaev superexchange.

\subsubsection{Heisenberg-Kitaev (HK) model}

Chaloupka et. al. derived the spin-Hamiltonian for honeycomb lattice iridate $\mathrm{A}_{2} \mathrm{IrO}_{3}, \mathrm{~A}$ $=\mathrm{Na}$, Li.[13] Here $\mathrm{Ir}^{4+}$ ion in the octahedral crystal field and strong SOC realize $\mathrm{J}_{\text {eff }}=$ $1 / 2$ Krammers state. Now these extended Kramers doublets (which is considered all in this thesis as $S=1 / 2$ of Ir) in the $90^{\circ}$ Ir-O-Ir bonding geometry can have direct exchange via overlap of the orbitals with the Kitaev exchange. So the total spin Hamiltonian for $\mathrm{A}_{2} \mathrm{IrO}_{3}$ with the nearest neighbor $(\mathrm{NN})$ interactions is given by:

$$
\begin{array}{r}
H=-K \sum_{<i, j>\gamma} S_{i}^{\gamma} S_{j}^{\gamma}+J_{1} \sum_{<i, j>} \mathbf{S}_{i} \cdot \mathbf{S}_{j} \\
H_{H K}=-2 \alpha \sum_{<i, j>\gamma} S_{i}^{\gamma} S_{j}^{\gamma}+(1-\alpha) \sum_{<i, j>} \mathbf{S}_{i} \cdot \mathbf{S}_{j}
\end{array}
$$

Here first term represents Kitaev exchange via three distinct NN bonds referred as $\gamma(=x, y, z)$ and $K$ is the Ising-like ferromagnetic (FM) coupling between the NN Ir-spins. The second term represents classical antiferromagnetic (AF) Heisenberg exchange between nearest neighbor spins where $J$ is the AF spin-coupling. $J$ and $K$ were derived from multiband Hubbard model in Ref. [13]. To determine a phase diagram, Eqn. 2.8 is parametrized as $K=2 \alpha$ and $J=1-\alpha$. Parametrized HK model is given in Eqn. 2.9. The whole phase diagram was studied in the parameter range $0<\alpha<1$.

At $\alpha=0$ only Heisenberg term contributes, HK model shows a Néel Ground state. At the opposite limit $\alpha=1$ Eqn. 2.9 corresponds to the exactly solvable Kitaev model with 
short-range spin liquid state.[11] This model also obtains another exactly solvable ground state at $\alpha=1 / 2$. It is stripy AF state with spins polarized in the direction of one of the crystallographic axis. Hence HK model gives three ground states shown in phase diagram in Fig. 2.7. The dotted vertical line in the phase diagram indicates the boundary between different ordered phase and represents zero-energy line of the spin-wave (SW) spectra. At this position, second derivative of the ground state energy $\left(E_{G S}\right)$ shows singularity hence a infinite degeneracy exists at this boundary.[13]
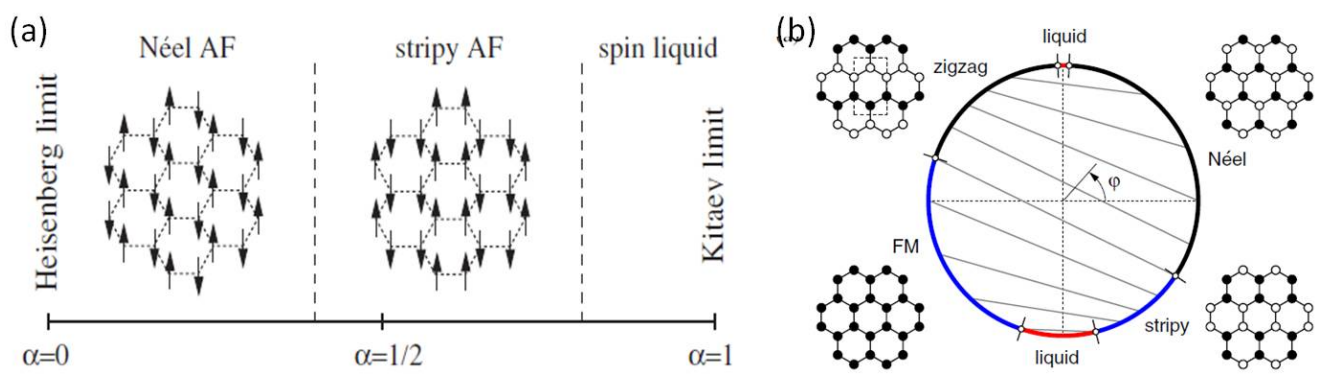

Figure 2.7: (a) Phase diagram of Heisenberg-Kitaev (HK) model with AF Heisenberg and FM Kitaev, reprinted from [13] (b) Phase diagram of the generalized HK model containing all four combination of FM and AF ; Kitaev and Heisenberg interaction, reprinted from[33] In the magnetic structures ( stripy, zig-zag etc.) open and solid circles indicate up and down spins respectively. The rectangular box in the zigzag pattern (top-left) shows the magnetic unit cell.

Chaloupka et. al. proposed a more general form of HK model, given in Eqn. 2.10.[33] First term represents bond dependent NN Kitaev exchange interaction of Ising type and the second term represents isotropic Heisenberg interaction.

$$
\begin{array}{r}
H_{i j}^{\gamma}=2 K S_{i}^{\gamma} S_{j}^{\gamma}+J \mathbf{S}_{i} \cdot \mathbf{S}_{j} \\
H_{i j}^{\gamma}=A\left(2 \sin \varphi S_{i}^{\gamma} S_{j}^{\gamma}+\cos \varphi \mathbf{S}_{i} \cdot \mathbf{S}_{j}\right)
\end{array}
$$

In this generalized model (Eqn. 2.10) both $K$ and $J$ can have positive and negative value, representing AF and FM exchange interaction respectively. Hence $K$ and $J$ are parametrized in the polar-space. New energy scale parameters are $\mathrm{A}=\sqrt{K^{2}+J^{2}}$ and $\varphi, K=A \sin \varphi$ and $J=A \cos \varphi$. Parametrized generalized HK model is given in Eqn. 2.11. Phase diagram will have four coordinates having four combination of $K$ and $J$.

(i) For $0 \leq \varphi \leq \pi / 2: J$ and $K$ both are positive, AF Heisenberg and Kitaev exchange which favor Néel correlation having Néel $\operatorname{AF}$ ground state at $\varphi=0$ and a new spin liquid ground state near $\varphi=\pi / 2$ governed by AF Kitaev exchange where $K=-J$ at the spin liquid boundary. (ii) In the second coordinate for $\pi / 2 \leq \varphi \leq \pi$ : $K$ is AF but $J$ is FM 

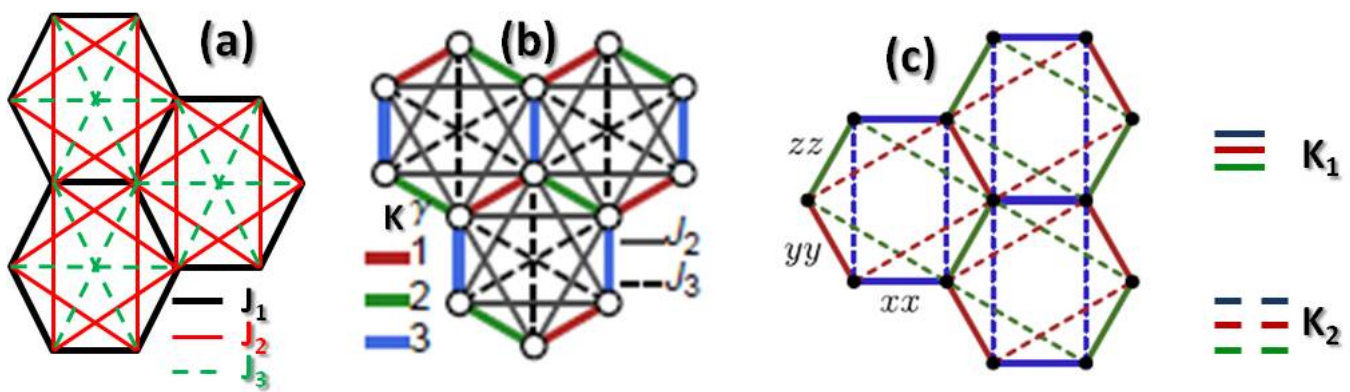

Figure 2.8: (a) Nearest neighbor (NN) and beyond NN Heisenberg exchange interactions in honeycomb lattice for $J_{1}-J_{2}-J_{3}$ model described in Eqn. 2.12. (b) All exchange interactions for $J_{2}-J_{3}$ Heisenberg-Kitaev model described in Eqn. 2.13, reprinted from [34]. (c) NN $\left.\left(K_{1}\right)\right)$ and $2^{\text {nd }} \mathrm{NN}\left(K_{2}\right)$ Kitaev exchange for the $2^{\text {nd }}$ neighbor HK model described in Eqn. 2.15, based upon Ref. [35].

which leads to a new ordered ground state called zig-zag state near $\varphi=3 \pi / 4$. Now hopping process in this region is governed by interorbital NN $t_{2 g}-e_{g}$ hopping via $90^{\circ} \mathrm{Ir}-\mathrm{O}$-Ir exchange path instead of a $t_{2 g}-t_{2 g}$ hopping. After a certain increase of FM Heisenberg term, a FM state is favored. Boundaries of all these phases were determined from exact diagonalization and singularity in the second derivative of $E_{G S}$ by Chaloupka et. al.[33] (iii) In the FM Kitaev and FM Heisenberg limit $\pi \leq \varphi \leq 3 \pi / 2$ : FM spin-correlation is favored, only at the boundary at $\varphi=-\pi / 2$ or $3 \pi / 2$ FM Kitaev spin liquid state is realized same as described by A. Kitaev [11]. (iv) $3 \pi / 2 \leq \varphi \leq 2 \pi$ parameter region: Kitaev exchange $K$ is FM and Heisenberg exchange $J$ is AF same like HK model in Eqn. 2.8. Hence at $\varphi=-\pi / 4$, the AF-stripy phase is realized with same exchange process as in first HK model. Uniquely this model gives zig-zag phase and a AF Kitaev spin-liquid state mediated by $\mathrm{NN} t_{2 g}-e_{g}$ superexchange process.

\subsection{2 $J_{1}-J_{2}-J_{3}$ model}

Kitaev superexchange process occurs due to $90^{\circ}$ Ir-O-Ir superexchange path. But in a honeycomb lattice if this Ir-O-Ir bonding angle severely deviates from $90^{\circ}$ due to trigonal distortion then Kitaev exchange will be absent and exchange interaction will solely be governed by Heisenberg exchange process. In such cases, isotropic $2^{\text {nd }}$ and $3^{\text {rd }}$ nearest neighbor Heisenberg exchange $J_{2}$ and $J_{3}$ can play important role along with the nearest neighbor direct exchange $J_{1}$. There are several $J_{1}-J_{2}$ model on honeycomb lattice with same type of sign and different sign. But one can not exclude a $J_{3}$ at least for a spatially extended 5d- orbitals. So a total isotropic spin-hamiltonian has been investigated with all the three neighbor Heisenberg interaction given in Eqn. 2.12.[36] 


$$
H=J_{1} \sum_{<i, j>} \mathbf{S}_{i} \cdot \mathbf{S}_{j}+J_{2} \sum_{<<i, k>>} \mathbf{S}_{i} \cdot \mathbf{S}_{k}+J_{3} \sum_{<<<i, l>>>} \mathbf{S}_{i} \cdot \mathbf{S}_{l} .
$$

Now the coupling constants $J_{i}$ can be either $\operatorname{AF}\left(J_{i}>0\right)$ or FM $\left(J_{i}<0\right)$. Depending on the values of the parameter $J_{i}$, different classical ground states are obtained. Detail study of the phase diagram for classical $J_{1}-J_{2}-J_{3}$ model was done by E.Rastelli and a detail $J_{3}-J_{2}$ phase diagram obtained (Fig. 6.3). [37, 38] Same phase diagram was investigated by Fouet et. al. including a search of different quantum phases.[39] Choi et. al. investigated $J_{1}-J_{2}-J_{3}$ model in detail including derivation of spin-wave spectra for all the AF spin-coupling. [36] This model shows really rich phase diagram mediated by frustrated exchange interactions: (i) three AF collinear phase Néel (all the NN of a spin are antiparallel ), stripy (two of the $\mathrm{NN}$ are antiparallel and one NN is parallel ) and zig-zag (two of the NN are parallel and one NN is antiparallel ) (ii) Two helical phase or spiral phase (iii) one FM phase with all the NN parallel spins. [37, 38]

\subsection{3 $J_{2}-J_{3}$ Heisenberg-Kitaev model}

In honeycomb lattice iridates, near $90^{\circ}$ Ir-O-Ir bonding angle always keeps a clear path for anisotropic Kitaev exchange. Hence Kimchi et. al. proposed $J_{2}-J_{3}$ Heisenberg-Kitaev model with beyond nearest neighbor exchange interaction given in Eqn. 2.13.[34] All the isotropic Heisenberg interactions and three bond dependent Kitaev interactions $(\gamma=1,2,3)$ are shown in Fig. 2.8(b). Hence, this model exhibits both geometrical frustration of $J_{1}, J_{2}$ and $J_{3}$ and dynamical frustration anisotropic FM Kitaev exchange. Phase diagram must be much richer than so far described model for honeycomb lattice. The Hamiltonian is given by:

$$
H=-K \sum_{<i, j>\gamma} S_{i}^{\gamma} S_{j}^{\gamma}+J_{1} \sum_{<i, j>} \mathbf{S}_{i} \cdot \mathbf{S}_{j}+J_{2} \sum_{<<i, k>>} \mathbf{S}_{i} \cdot \mathbf{S}_{l}+J_{3} \sum_{<<<j, l>>>} \mathbf{S}_{j} \cdot \mathbf{S}_{l}
$$

This can also be parameterized with $\alpha$ like in Eqn. 2.9 and $J_{i}$ and $K$ can be written in the units of $J$ and the resulting Hamiltonian is given in Eqn. 2.14. This can also be parameterized with $\alpha$ like in Eqn. 2.9 and $J_{i}$ and $K$ can be written in the units of $J$ and the resulting Hamiltonian is in Eqn. 2.14.

$$
H=J\left[-2 \alpha \sum_{<i, j>\gamma} S_{i}^{\gamma} S_{j}^{\gamma}+(1-\alpha)\left(\sum_{<i, j>} \mathbf{S}_{i} \cdot \mathbf{S}_{j}+J_{2} \sum_{<<i, k>>} \mathbf{S}_{i} \cdot \mathbf{S}_{l}+J_{3} \sum_{<<<j, l>>>} \mathbf{S}_{j} \cdot \mathbf{S}_{l}\right)\right]
$$


They investigated mainly the ordered phase in the vast region of the phase diagram. Lowest energy magnetic configuration was determined for each point $\left(\alpha, J_{2}, J_{3}\right)$. Ordered phase diagram was obtained for different $\alpha$ in $J_{3}-J_{2}$ coupling-space for AF coupling. Main striking feature is that all the ordered phases of $J_{1}-J_{2}-J_{3}$ model is realized in $J_{2}-J_{3} \mathrm{HK}$ model in much smaller parameter space. For $\alpha=0.3$, all the ordered phases Néel, stripy, zig-zag and spiral phase exist in the parameter range $0 \leq\left(J_{2}, J_{3}\right) \leq 1$. This model may give a broader playground for honeycomb lattice iridates and rhodates. Kitaev spin-liquid phase was also observed for $\alpha \geq 0.8$ for small $J_{2}$ and $J_{3}$.

\subsection{4 $2^{\text {nd }}$ neighbor Heisenberg-Kitaev model}

Recently Reuther et. al. proposed another extension of the Heisenberg-Kitaev model including next-nearest neighbor (NNN) Heisenberg and Kitaev exchange with the nearest neighbor (NN) HK model described in the Eqn. 2.15. [35] Here NN Heisenberg exchange is AF $J_{1}\left(J_{1}>0\right)$ and Kitaev exchange $K_{1}$ is FM $\left(K_{1}<0\right)$ same as described in Eqn. 2.8. NNN Heisenberg exchange is $\mathrm{FM}\left(J_{2}<0\right)$ and Kitaev exchange is $\mathrm{AF}\left(K_{2}>0\right)$. NN and NNN Kitaev couplings are shown in Fig. 2.8(c).

$$
H=J_{1} \sum_{<i, j>} \mathbf{S}_{i} \cdot \mathbf{S}_{j}+K_{1} \sum_{<i, j>\gamma} S_{i}^{\gamma} S_{j}^{\gamma}+J_{2} \sum_{<<i, k>>} \mathbf{S}_{i} \cdot \mathbf{S}_{k}+K_{2} \sum_{<<i, k>>\gamma} S_{i}^{\gamma} S_{k}^{\gamma}
$$

NNN exchange in the $2^{\text {nd }}$ neighbor HK model involved with $2^{\text {nd }}$ neighbor hopping between Ir isospins. Shitade et. al. derived NNN hopping integral for $\mathrm{Na}_{2} \mathrm{IrO}_{3}$.[14] The transfer integral between a NNN pair depends on spin. The wave function for the isospin is complex so hopping integral will also have a complex and real part. The complex transfer integrals are asymmetric and they have different component along the different cubic $x-y-z$ axis. The real part is the direct $d d$ hopping between NNN pairs. In the Mott limit, these bond selective spin-orbit hoppings (complex part) corresponds to a second neighbor coupling, $J^{\prime}>0$. The Hamiltonian for NNN coupling is given by:[35]

$$
H_{N N N}=\sum_{<<i, j>>, \gamma} J^{\prime}\left(-\mathbf{S}_{i} \cdot \mathbf{S}_{j}+2 S_{i}^{\gamma} S_{j}^{\gamma}\right)
$$

First term represents FM Heisenberg coupling and the second term is AFM Kitaev coupling. Exchange coupling in Eqn. 2.16 is due to second neighbor hopping of Ir-spin-orbitals described by Shitade et. al.[14] There is also real second-neighbor AF Heisenberg exchange ${ }^{1}$ given by $J_{0}^{\prime}$. So net second neighbor Heisenberg coupling is $J_{2}=J_{0}^{\prime}-J^{\prime}$. Now considering

\footnotetext{
${ }^{1}$ derived from real part of the second order hopping
} 


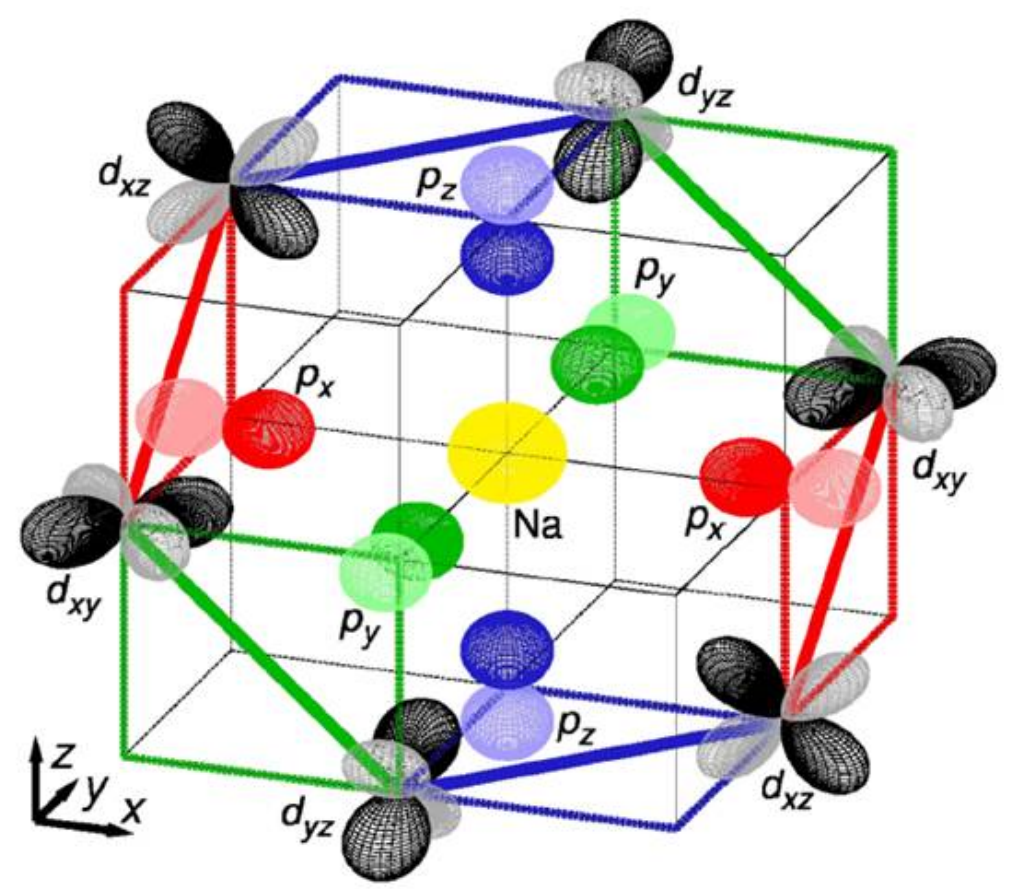

Figure 2.9: Most relevant $\mathrm{O}$ p-assisted hopping paths in idealized honeycomb structure, reprinted from [40].

imaginary part of the second neighbor hopping (J') small compared to real part $\left(J_{0}^{\prime}\right), J_{2}<0$ which implies a FM Heisenberg coupling.[35] NNN Kitaev coupling is $K_{2}=2 J^{\prime}$.

\subsection{Itinerant picture of Honeycomb lattice iridates and rho- dates}

In the strong SOC limit localized $J_{\text {eff }}$ states form under this consideration : $W<\left(J_{H}, \lambda\right)<$ $U$. But in a extended $5 d$ orbital, single electron hopping $t$ would be also an important factor which could give itinerant description of Honeycomb lattice iridate. Mazin et. al. took this approach to describe electronic structure of $\mathrm{Na}_{2} \mathrm{IrO}_{3}$. In their approach they had first calculated tight-binding (TB) parameters and identified nearest neighbor (NN) O-assisted hooping between unlike 5d-orbitals as the leading hopping channel (see in Fig. 2.9).[40]

Three different types of NN Ir-O-Ir hopping are allowed via three different bonds (shown in three different colors in Fig. 2.9). Along $x y$-bond (blue) hopping is only allowed between $d_{x z}$ and $d_{y z}$ orbitals via $\mathrm{O} p_{z}$ orbital, along the $x z$ bond (green) hopping occurs between $d_{y z}$ and $d_{x y}$ orbitals via $\mathrm{O} p_{y}$ orbital and along $y z$ bond (red) hopping is allowed between $d_{x y}$ and $d_{x z}$ via $\mathrm{O} p_{x}$ orbital. This hopping is named $t_{1}^{\prime}$. Also they considered NNN hopping via $\mathrm{Na} 3 s$ orbital which is also bond dependent. Now lets see how this NN O-assisted hopping $t_{1}^{\prime}$ leads to a semi-itinerant picture. Lets consider an electron on a given Ir site at $d_{x z}$ orbital. 
This site has three NN orbital but electron in $d_{x z}$ orbital can hop to neighboring orbital with $d_{y z}$ symmetry only located at a particular NN site. From $d_{y z}$ orbital it can futher hop to the $d_{x y}$ state at the next site and so on. Following this hopping, after six hopping the electron will reach to the same $d_{y z}$ orbital, from where it started. Hence the electron is equally localized to the six sites forming a hexagon. In other words electron is de-localized within the whole hexagon instead of localizing to a single site. Such a hexagon state could be called a molecular orbital. In classical molecular orbitals, electron is localized on spatially separated molecules and here electron is localized within one Ir-hexagon. A hopping between two adjacent hexagon is forbidden by bond dependent hopping. This semi-localized unit is called "Quasi Molecular Orbital".

By considering this NN hopping, NNN hopping and SOC, band structure of $\mathrm{Na}_{2} \mathrm{IrO}_{3}$ was calculated by Mazin et. al. Coulomb repulsion $U$ was also considered to get proper band structure. But obviously this description was given in the consideration $U<<t$. So it gives a different insight of honeycomb iridate than the SO Mott insulating picture. 


\section{Chapter 3}

\section{Experimental Techniques}

\subsection{Sample Preparation}

In crystalline solid state physics two types of samples are prepared: polycrystalline material and single crystalline material. In principle polycrystalline material can be synthesized for all the materials for which a single crystalline phase exist but the vice verse is not true. Basic difference between them is in their grain size. Usual grain size of polycrystal varies from few nanometer to 1-10 micrometer. Whereas a single crystal can be grown of the size from 50-100 micrometers to few centimeters depending upon the preparation method.

\subsubsection{Polycrystalline material}

Probably the most widely used method to prepare polycrystalline material is solid state reaction method. Solid materials usually do not react at room temperature in usual timescale. One has to heat them at certain high temperatures, often upto 1000 to $1050^{\circ} \mathrm{C}$, in order for reaction to happen at an appreciable rate. Hence for solid state reactions thermodynamic considerations are important which determine whether a resaction will occur at a certain temperature or not depending on change in the free energy. As well as kinetic factors are important to determine the rate of the reactions.[41] Before performing a solid state reactions, following factors need to be considered: Reaction conditions: temperature, pressure and environment; Structural conditions: possibility of crystallization of the desired phase in a allowed crystal structure and the reaction rate which controls the interdiffusion rate of different reactant solids. If above three conditions allow to react then the solid state reactions happen. 
For solid state reaction all the solid materials are mixed in agate mortar and pestle or in a ball-mill for several hours. Sometimes a liquid medium like alcohol or acetone are used for homogeneous mixing. Well mixed reagent are put in a crucible to be heated in a furnace upto a high temperature. For a good solid state reaction, following things need to be carefully considered:

- Starting reagents need to be very pure and they should be taken in stoichiometric proportion because extra phases can not be removed like flux.

- To maximize the surface area of the reactants, fine powder is used and they are very well grinded and mixed for several hours.

- One should know reactivity of the reagents beforehand. Based upon this, environment and crucibles are chosen. For normal conditions alumina $\left(\mathrm{Al}_{2} \mathrm{O}_{3}\right)$ crucible and open atmosphere are chosen usually. But sometimes due to reactivity, certain metal crucibles (e.g. Platinum) are needed to be used. For special environment, sometime reactions are done inside the sealed quartz tube ( below $1300^{\circ} \mathrm{C}$ ) or in a special environment inside a furnace.

- One should know range of temperature where the desired phase is stable. Reaction should be done below the maximum limit of the temperature and should be quenched above the minimum limit of the temperature. Otherwise, either a secondary phase forms or the desired phase decompose at high temperature.

\subsubsection{Sample preparation in special environment}

For stable oxide phases, reaction in a open furnace is sufficient. In some transition metal oxides valence state is not the most energetically favored one. For those cases, one needs to control oxygen during synthesis. During this thesis work such special environment was needed for $\mathrm{Li}_{2} \mathrm{RhO}_{3}$.

$\mathrm{Li}_{2} \mathrm{RhO}_{3}$ was synthesized in a horizontal furnace where oxygen was flowed continuously during reaction. Fig. 3.1(a) shows this furnace where the directions of gas flow are shown. For charge carrier doping to obtain a stable gas atmosphere, a 5 Bar overpressure furnace (Fig. $3.1 \mathrm{~b}$ ) was designed by modifying the horizontal flow furnace by the help of Prof. Winzer and our institute workshop.

This furnace can hold upto 5 Bar of overpressure of a desired gas atmosphere (e.g Oxygen, Argon etc.) and above that pressure safety valve opens and reduces the pressure 


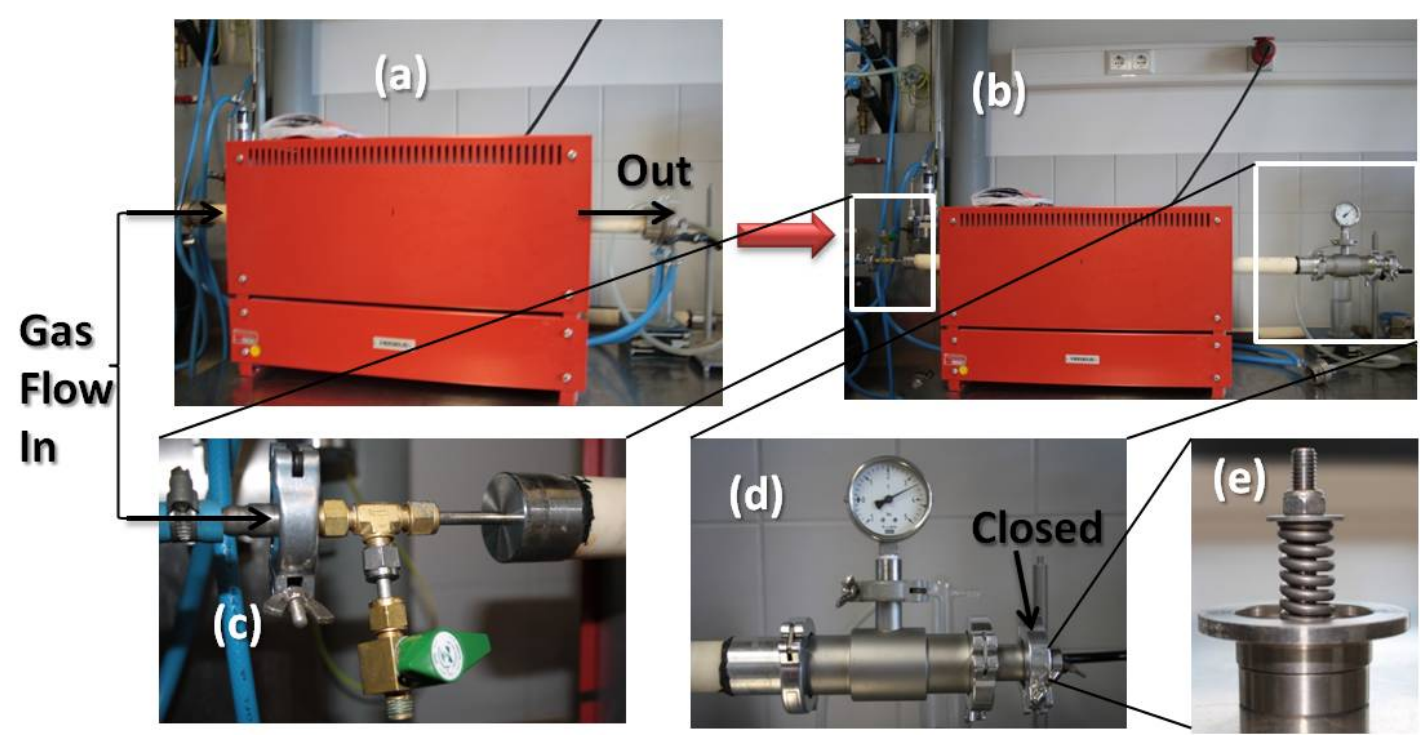

Figure 3.1: (a) Gas flow horizontal furnace, gas in and out directions are shown. (b) Modified to a fixed atmosphere (Oxygen, Argon, Nitrogen etc.) closed furnace upto 5 bar overpressure.(c) Closer look to the gas inlet and venting path in the closed furnace. (d) Closer look to the other end of the closed furnace where Barometer and high pressure safety valve [shown in (e)] are attached.

(Fig. $3.1 \mathrm{~d}$ and e). This safety valve was designed in workshop. The upper pressure limit depends upon the strength of the spring attached. By compressing the spring one can increase the upper limit and by relaxing the spring one can reduce the limit (Fig. 3.1 e). Upper limit is always kept below 5 Bar for the safety of the alumina tube of the furnace. To release the pressure a parallel ventil is attached near the gas inlet (Green ventil in Fig. $3.1 \mathrm{c}$ ) which can immediately pass out the gas inside. This furnace was used for oxygen annealing of $\mathrm{Li}_{2} \mathrm{RhO}_{3}$ at 3 bar pressure. Future plan is to use this furnace in argon atmosphere for charge carrier doping where transition metal valence state needs to be changed.

\subsubsection{Single crystal growth}

In polycrystalline material few $\mu \mathrm{m}$ size crystals are randomly oriented and appear as powder. In some cases it is needed to study directional dependence of the magnetic, electrical and thermodynamic property which can not be measured without a single crystal. Single crystal is the most significant pillar of the solid state physics. Technological value of a big single crystal is enormous, specially in semiconductor industry. Hence quest for good single crystal is going on from very ancient time. Earliest manifestation of single crystal is the crystal growth of salt and sugar from supersaturated solutions. Crystal growth methods can be classified by three type of phase transitions: 
(1) Solid-Solid : solid crystal grows from solid phase.

(2) Liquid-Solid: solid crystal grows from liquid melt or solution by change of temperature and pressure.

(3) Gas-Solid: solid crystal grows from vapor by sublimation-condensation.

\subsubsection{Solid growth}

In the solid growth method, the crystals are grown from polycrystalline or amorphous solid by atomic diffusion. The atomic diffusion is a very slow process so that it needs to be sintered or annealed at a high temperature for a long time. Popular mechanisms for solid method crystal growth are the recrystallization process, the strain-anneal method, the solid-state electrolysis and the thin layer growth: solid-state epitaxy etc.[42] When a polycrystalline sample is isothermally annealed at high temperature, several grains may nucleate. This nucleation is initiated due to temperature gradient or some vapor pressure. The nucleated grains try to reduce the free energy by reducing number of grain boundaries. So some favored grains will grow in size by slowly moving the grain boundaries which results in growth of the crystal. The grains can grow in very random direction which can also change very suddenly, hence the crystals grow in this method in very random direction attached with one another in very random angles. One benefit of this method is absence of any foreign phase with crystals like flux and another disadvantage is presence of defects due to random change of growth direction.

\subsubsection{Liquid growth}

Most extensively used and most popular method of crystal growth is the liquid growth. In this method, a solid crystal is grown from liquid solution or melt by slow change of the temperature. Different popular mechanisms are: (i) Melt growth where crystal is grown from molten material by decrease of temperature e.g. Bridgman-Stockbarger, Czochralski and zone melting etc. This method is extensively used for growing big single-crystals. (ii) Flux method where solid materials dissolve in a flux reagent which is solid in room temperature but liquid in a higher temperature. Then a nucleation happens in the supersaturated solution of flux and the solid above the crystal growth temperature. After that a slow cooling increases supersaturation of the flux-solid solution which leads to increase in size of the nucleated crystal. This increase in size continues to happen upto little below the crystal growth temperature. Sometimes the solid material itself acts as a flux and little 
off-stoichiometry in any of the material initiate the nucleation at the high temperature. This method is called self - flux method. One of the main challenge in the flux method is separating crystals from flux. Different methods are used for that: dissolving in any strong solution like acid or base where the solid crystal does not dissolve but flux dissolves. Some halide fluxes used which are used in oxide crystal growth dissolve in water or alcohol also. Metallic fluxes are separated by centrifuge method above melting point of the flux. The flux method of crystal growth is most widely used and earliest method of crystal growth. Other popular methods of crystal growth from liquid are the sol-gel method and the hydrothermal method where a ambient pressure plays an important role.[43]

\subsection{Elemental analysis}

After growing single or poly-crystal, first characterization should be verifying the stoichiometry of the phase i.e. elemental quantification of the different elements inside the crystal. The elemental analysis confirms purity of the phase, absence of any flux as foreign phase.

\subsubsection{Energy-dispersive X-ray}

In the energy dispersive X-ray spectrometry (EDX) method, elemental quantification is done from characteristic X-rays spectrum emitted due to bombardment of a high energy focused electron beam $\left(E_{i}=20 \mathrm{keV}\right)$. All elements from atomic number $Z=4(\mathrm{Be})$ to $92(\mathrm{U})$ can be detected in principle by EDX though it is not best for $Z<10$. All the elements have very different x-ray spectral lines $\left(K_{\alpha}, L_{\alpha}, M_{\alpha}, K_{\beta}\right)$ at different energies. Characteristic Xrays result from electron transitions between inner orbits which is very different for different elements having different electron configuration. Sometimes some lines of different elements overlap and make it little bit tough to be detected. Quantification is done from the intensity of the lines comparing with the calibration. It is a very local probe so homogeneity of the composition within a crystal can be determined very accurately.[44]

\subsubsection{Inductively coupled plasma mass spectrometry (ICPMS)}

Mass spectrometry (MS) is a quantification technique which quantifies atoms, isotopes, molecules in a material by mass to charge ratio. Due to this unique mechanism MS is much more sensitive than any other quantification technique. It is able to detect elements like $\mathrm{Li}, \mathrm{H}$ which can not be detected by EDX. MS is a very useful quantification technique 
in organic chemistry and biology where complex but light bio-molecules e.g proteins and peptides need to be detected very accurately. In the inductively coupled plasma mass spectrometry (ICPMS), first a plasma of different ions is created by laser heating and it is channelized mixed with argon gas through a quadrupole to separate different ions. In the mass spectrometer detector different ions are detected and then relative quantification is done comparing with specimen data for each atom.

\subsection{Structural characterization: X-ray diffraction}

Crystals are regular array of atoms in three dimensions. Hence different periodic planes of atoms having different periodicity can be characterized by vector geometry. In a 3D crystal periodic planes, are characterized by $(h k l)$ planes. W. L. Bragg and W. H. Bragg gave pioneering theory of X-ray diffraction, they stated that as wavelength of x-ray and the periodicity of the $(\mathrm{hkl})$ planes are of same order, these periodic crystal planes (consisting of $2 \mathrm{D}$ array of atoms) can constructively diffract x-ray which will give rise to Bragg's reflection governed by Bragg's law:

$$
2 d \sin \theta=n \lambda
$$

Where $d$ is the periodicity of the $(h k l)$ planes or their distances. $\lambda$ is the wavelength of the $\mathrm{x}$-ray and $n$ is the order of reflection, $n \in[1,2,3 \ldots]$. Hence different $(h k l)$ planes in a crystal give constructive Bragg's diffraction at different angles $\theta$ for a fixed incident x-ray beam. So a $\theta-2 \theta$ scan of a crystal by x-ray gives all sets of Bragg's reflection at different $2 \theta$ and these whole set of reflections are characteristics of different crystal structures. Intensity profile depends upon structure factor of the the reflecting atoms. Hence accumulating all these data one can identify a crystalline phase very accurately.[45] One of the successful method for fitting a experimental x-ray diffraction pattern to characterize a crystalline phase in a particular crystal structure is Rietveld refinement. Rietveld refinement is done using GSAS.[46, 47]

\subsection{Magnetization Measurement}

In a magnetic material, spins arrange themselves in a certain direction avoiding thermal fluctuations at low temperature and this give rise to net magnetic moment to the magnetic material. Under a static magnetic filed $H$ this net magnetization can be measured. One of 
the the efficient method to measure this magnetization is superconducting quantum interference device (SQUID). In SQUID, magnetization is measured by the trapped magnetic flux inside a superconducting loop of two Josephson junction. This unique technique increases the sensitivity of the magnetization measurement by few orders to $10^{-18} \mathrm{~T}$. Very small magnetic phase transition can be easily detected by this method. We have used commercially designed SQUID device by QUANTUM design : MPMS XL.[48] For magnetization measurement, samples are put in a small plastic capsule which is centered in a plastic straw of 20 $\mathrm{cm}$ length. Background from straw and capsule is diamagnetic and two order of magnitude smaller than samples measured. Still, when very small amount of samples are measured this background contribution is well taken care of. We have measured static Magnetization (M) by reciprocating sample option (RSO) in MPMS in the $\mathrm{G}-\mathrm{cm}^{3}$ unit. Hence molar susceptibility $(\chi)$ is calculated by following relation:

$$
\chi=\frac{M M_{w}}{H m}
$$

Where $M_{w}$ is the molecular weight of the magnetic materials, $m$ is the mass of the sample in gram and $H$ is the applied magnetic field in Oersted. $\chi$ is obtained in $\mathrm{cm}^{3} / \mathrm{mol}$ unit. The ac susceptibility can also be measured by applying an ac field having very low amplitude and frequency range $1-1500 \mathrm{~Hz}$ in MPMS. The first $\left(\chi^{\prime}\right)$ and second harmonics $\left(\chi^{\prime \prime}\right)$ of the ac susceptibility can be directly obtained in MPMS.[48]

\subsection{Heat Capacity}

Heat capacity is a very useful thermodynamic tool to detect any magnetic and structural phase transition. The heat capacity is defined by:

$$
C_{x, y, \ldots}=\left(\frac{d Q}{d T}\right)_{x, y, \ldots}
$$

Where $x, y$ are the parameters which are kept constant to measure the change of temperature. We have measured the heat capacity in relaxation method by commercial physical property measurement system (PPMS) of Quantum Design.[49] Quasi-adiabatic condition is achieved inside a high vacuum chamber with pressure $10^{-6}$ mbar [50] where the heat capacity is measured at a constant pressure in the temperature range $0.5 \mathrm{~K}$ to $400 \mathrm{~K}$. Heat capacity is given by:

$$
C_{p}=\left(\frac{d Q}{d T}\right)_{p}
$$


For a solid state material heat capacity has three part : (i) lattice heat capacity which can be theoretically explained by Debye theory (at low temperature i.e, far below Debye temperature $\left(\theta_{D}\right)$ of the sample, $\left.C_{\beta} \propto T^{3}\right)$, (ii) electronic heat capacity $\left(C_{e l} \propto T\right)$ and (iii) magnetic heat capacity $\left(C_{m} \propto T^{\alpha}\right)$. Hence total heat capacity at low temperature is given by:

$$
C_{p}=\gamma T+\beta T^{3}+A T^{\alpha}
$$

Where in the first term, $\gamma$ is Sommerfield's constant, representing electronic heat capacity, in the second term, $\beta$ is the Debye constant, representing lattice heat capacity and the third term represents magnetic heat capacity having a $\alpha$-power law dependence. For an insulator electronic heat capacity is zero and total heat capacity $\left(C_{p}\right)$ is given by $2 n d$ and $3 r d$ terms in Eqn. 3.5.

\subsection{Resistivity}

Resistivity of a solid material categorize it as an insulator or a metal. DC resistivity is measured in the temperature range $0.5 \mathrm{~K}$ to $400 \mathrm{~K}$ by four probe technique in commercial physical property measurement system (PPMS) of Quantum Design.[51] For resistivity measurement in an insulator contact resistance is not a big issue. So, I have used silver epoxy and $\mathrm{Cu}$-wire to make current and voltage contacts. 


\section{Chapter 4}

\section{Honeycomb lattice Iridate $\mathrm{A}_{2} \mathrm{IrO}_{3}$ $(\mathrm{A}=\mathrm{Na}$ and $\mathrm{Li})$}

In this chapter, synthesis procedure, physical property and underlying physics of the honeycomb lattice iridate compounds $\mathrm{A}_{2} \mathrm{IrO}_{3}\left(\mathrm{Na}_{2} \mathrm{IrO}_{3}, \mathrm{Li}_{2} \mathrm{IrO}_{3}\right)$ are described in full detail. Primary effort of my thesis was to synthesize a phase pure and highly ordered $\mathrm{A}_{2} \mathrm{IrO}_{3}$ compound. The physical properties of $\mathrm{A}_{2} \mathrm{IrO}_{3}$ are controlled by structural disorder which is described in the section Disorder effect in $\mathrm{A}_{2} \mathrm{IrO}_{3}$. For the first time, all the low temperature physical properties have been studied on well-ordered $\mathrm{Li}_{2} \mathrm{IrO}_{3}$ as well as microscopic properties have been studied by different scattering experiments e.g. elastic and inelastic neutron scattering and x-ray scattering. The honeycomb lattice iridates has been investigated by different surface sensitive techniques e.g. angle resolved photo emission spectroscopy(ARPES), scanning tunneling spectroscopy (STS) and different local magnetic probe techniques like nuclear magnetic resonance (NMR), electron spin resonance (ESR) and muon spin rotation ( $\mu$-SR) etc. Some of these special experiments are discussed later in this chapter.

\section{1 $\quad \mathrm{A}_{2} \mathrm{IrO}_{3}$ crystal structure}

$\mathrm{A}_{2} \mathrm{IrO}_{3}$ crystallizes in a monoclinic crystal structure. Earlier reports from chemists had suggested both the $\mathrm{C} 2 / \mathrm{m}$ (Space group number 12) and $\mathrm{C} 2 / \mathrm{c}$ (Space group number 15) space group for $\mathrm{A}_{2} \mathrm{IrO}_{3}$. Later in the structural analysis basic differences between the two is discussed. The basic structure of $\mathrm{A}_{2} \mathrm{IrO}_{3}$ is shown in Fig 4.1. The structure is quasi 2D in nature, which is formed by alternative stacking of $\mathrm{AIr}_{2} \mathrm{O}_{6}$-layer and A-layer along $c$-axis. Fig 4.1(a), a view along $a b$-plane shows this stacking of alternating layers. Due to its monoclinic 
nature, the stacking is not parallel, one of the crystallographic angle is more than $90^{\circ}$. Fig

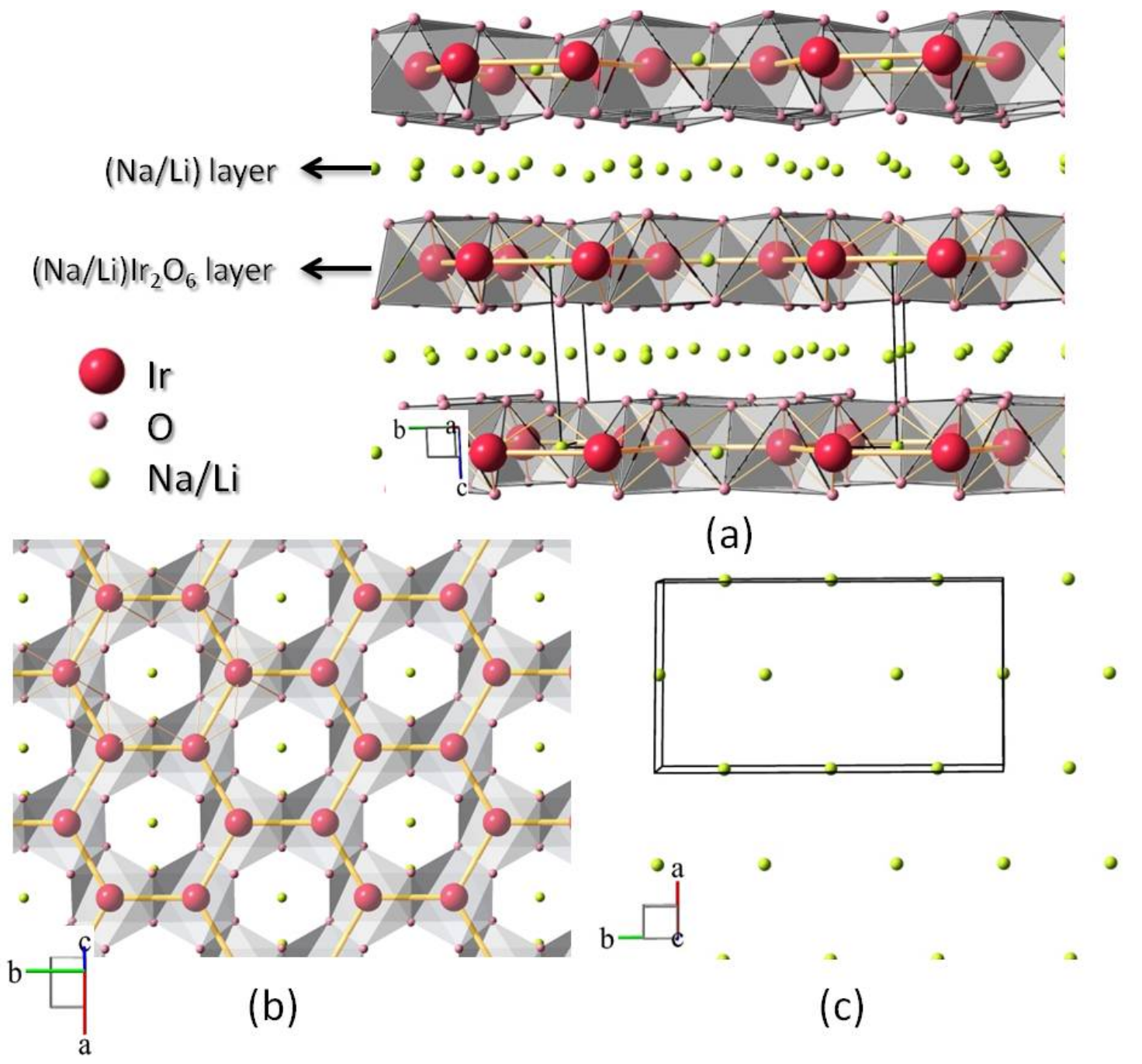

Figure 4.1: Crystal structure of $\mathrm{A}_{2} \mathrm{IrO}_{3}(\mathrm{~A}=\mathrm{Na} / \mathrm{Li})$. ( $\left.\mathrm{Na} / \mathrm{Li}\right)$, Ir and $\mathrm{O}$ atoms are shown in red, pink and green respectively. (a) The view of the structure along ab-plane (b) The view of the $\mathrm{AIr}_{2} \mathrm{O}_{6}$ layer along c axis. (c) The view of A layer along c axis.

4.1(b) and (c) show the view of the structure along the c-axis of $\mathrm{AIr}_{2} \mathrm{O}_{6}$ layer and $\mathrm{A}$ layer respectively. $\mathrm{AIr}_{2} \mathrm{O}_{6}$ is the honeycomb arrangement of edge sharing $\mathrm{IrO}_{6}$ octahedra and a $\mathrm{A}(\mathrm{Na} / \mathrm{Li})$ atom sitting at the center of the honeycomb. Each $\mathrm{IrO}_{6}$ octahedra are connected with the three other neighboring octahedra. In the A-layer in between two honeycomb layers, A-atoms are arranged in a hexagonal lattice. 


\section{2 $\quad \mathrm{A}_{2} \mathrm{IrO}_{3}$ synthesis}

\subsection{1 $\quad \mathrm{A}_{2} \mathrm{IrO}_{3}$ polycrystal}

$\mathrm{Na}_{2} \mathrm{IrO}_{3}$ polycrystal was synthesized by solid state reaction method in an open furnace.[52] Starting materials $\mathrm{Na}_{2} \mathrm{CO}_{3}(99.995 \%$, Alfa aesar), Ir metal powder ( $\geq 99.99 \%$, Alfa aesar) were mixed in 1.05:1 ratio, grinded for several minutes before putting in a furnace in alumina $\left(\mathrm{Al}_{2} \mathrm{O}_{3}\right)$ crucible. Primary calcination reactions were done at $750^{\circ} \mathrm{C}$ and $800^{\circ} \mathrm{C}$ as described below:

$$
\mathrm{Na}_{2} \mathrm{CO}_{3}+\mathrm{Ir} \stackrel{\mathrm{O} 2}{\longrightarrow} \mathrm{Na}_{2} \mathrm{IrO}_{3}+\mathrm{CO}_{2}
$$

In this step $\mathrm{Na}_{2} \mathrm{CO}_{3}$ leaves $\mathrm{CO}_{2}$, Ir take surrounding $\mathrm{O}_{2}$ and reacted to form $\mathrm{Na}_{2} \mathrm{IrO}_{3}$. These two calcination steps were always performed below melting point of $\mathrm{Na}_{2} \mathrm{CO}_{3}\left(823^{\circ} \mathrm{C}\right)$ to avoid melting of $\mathrm{Na}_{2} \mathrm{CO}_{3}$ before reacting. To obtain a good quality well ordered polycrystal this pre-reacted sample was heat treated till $950^{\circ} \mathrm{C}$ in $50^{\circ} \mathrm{C}$ steps after repetitive grinding, mixing with acetone and pelletizing. Pelletizing was done using a workshop made cylindrical pelletizer of $1 \mathrm{~cm}$ diameter and $5 \mathrm{~cm}$ height with a pressure of 20MPa. Last step of $\mathrm{Na}_{2} \mathrm{IrO}_{3}$ preparation at $950^{\circ} \mathrm{C}$ sometime need more than one heat treatment to avoid partial disorder. The well ordered $\mathrm{Na}_{2} \mathrm{IrO}_{3}$ polycrystal was used for nuclear magnetic resonance (NMR) experiment.

$\mathrm{Li}_{2} \mathrm{IrO}_{3}$ polycrystal synthesis was previously reported by many solid state chemistry groups. [53, 54, 55] $\mathrm{Li}_{2} \mathrm{MO}_{3}(\mathrm{M}=$ Transition metals $)$ class of materials are a great interest of study as a potential candidate for Li ion batteries. So far, primary structural and electrochemical studies had been reported to investigate its efficiency as Li-ion battery material. Detail study of the magnetic property and other low temperature thermodynamic properties were not studied in much detail. Our interest was to study true magnetic ground state of $\mathrm{Li}_{2} \mathrm{IrO}_{3}$, which earlier studies reported to be paramagnetic down to $2 \mathrm{~K}$.

$\mathrm{Li}_{2} \mathrm{IrO}_{3}$ was prepared in solid state reaction method. $\mathrm{Li}_{2} \mathrm{CO}_{3}$ (99.95\%, Alfa aesar) and Ir metal powder ( $\geq 99.99 \%$, Alfa aesar) were used as starting material in 1.05:1 ratio. Melting point of $\mathrm{Li}_{2} \mathrm{CO}_{3}$ is $723^{\circ} \mathrm{C}$ so the calcination reaction were done at $700^{\circ} \mathrm{C}$ and $750^{\circ} \mathrm{C}$.

$$
\mathrm{Li}_{2} \mathrm{CO}_{3}+\mathrm{Ir} \stackrel{\mathrm{O} 2}{\longrightarrow} \mathrm{Li}_{2} \mathrm{IrO}_{3}+\mathrm{CO}_{2}
$$

The resultant sample $\mathrm{Li}_{2} \mathrm{IrO}_{3}$ was then grinded, mixed with acetone and pelletized for further reaction. Sintering was done in $50^{\circ} \mathrm{C}$ steps upto $1000^{\circ}$, where each step was sintered for 24 hours. It was not easy to prepare a magnetically and structurally well ordered 
$\mathrm{Li}_{2} \mathrm{IrO}_{3}$ polycrystal in this method. With best of our efforts first polycrystal samples obtained with this method, showed ordered structure as confirmed by x-ray diffraction but failed to have any long range magnetic ordering. Later, we did certain modification of the standard method. Four to five times heat treatment to the sample were done between temperature range of 950 to $1000^{\circ} \mathrm{C}$. Only then a magnetic ordering was obtained which confirmed well structural ordering.

$\mathrm{Li}_{2} \mathrm{IrO}_{3}$ best polycrystal was prepared in flux method which showed clear long range magnetic ordering. This is a new polycrystal preparation method proposed for layered oxide materials by us. After calcination reaction of $\mathrm{Li}_{2} \mathrm{IrO}_{3}$ at $700^{\circ} \mathrm{C}$ and $750^{\circ} \mathrm{C}, \mathrm{Li}_{2} \mathrm{IrO}_{3}$ powder is mixed with 7-8 times (in weight) of $\mathrm{LiCl}$ (Alfa aesar 99.99\%, ultra dry) inside a glove box. $\mathrm{LiCl}$ is highly hygroscopic and in any contact with air it absorbs lots of moisture, so grinding and mixing in air is not possible. Then this mixture was put in a big crucible $(5 \mathrm{~cm}$ diameter) and then it was put in a furnace for slow heat treatment. Heat treatment profile was:

- Heating from room temperature (RT) to $600^{\circ} \mathrm{C}$ at a rate of $100^{\circ} \mathrm{C} /$ hour.

- Heating from $600^{\circ} \mathrm{C}$ to $850^{\circ} \mathrm{C}$ at a rate of $30^{\circ} \mathrm{C} /$ hour.

- Stay at $850^{\circ} \mathrm{C}$ for 10 hours.

- Cooling till $600^{\circ} \mathrm{C}$ at a rate of $3^{\circ} \mathrm{C} /$ hour.

- Furnace cooling up to room temperature.

After taking out this final product excess $\mathrm{LiCl}$ flux was washed several times by distilled water and then dried on hot plate. X-ray diffraction(XRD) was done for phase purity and structural ordering of the sample. A very well ordered $\mathrm{Li}_{2} \mathrm{IrO}_{3}$ polycrystal was achieved. In a layered system structural ordering depends upon coherent ordering of alternating layers which depends upon the proper stacking of the layers without any stacking fault. A slow cooling helps in proper stacking of the layers. Though presence of light elements like Li or $\mathrm{Na}$ in the structure makes slow cooling difficult as light elements have high probability to go out of the structure due to long heat treatment which can result impurity phase. But slow cooling in a $\mathrm{Li}$ rich environment ( $\mathrm{LiCl}$ flux) solves this problem for $\mathrm{Li}_{2} \mathrm{IrO}_{3}$. By this method, best $\mathrm{Li}_{2} \mathrm{IrO}_{3}$ polycrystal was produced which was used for bulk property measurement and for neutron and x-ray scattering experiments. 


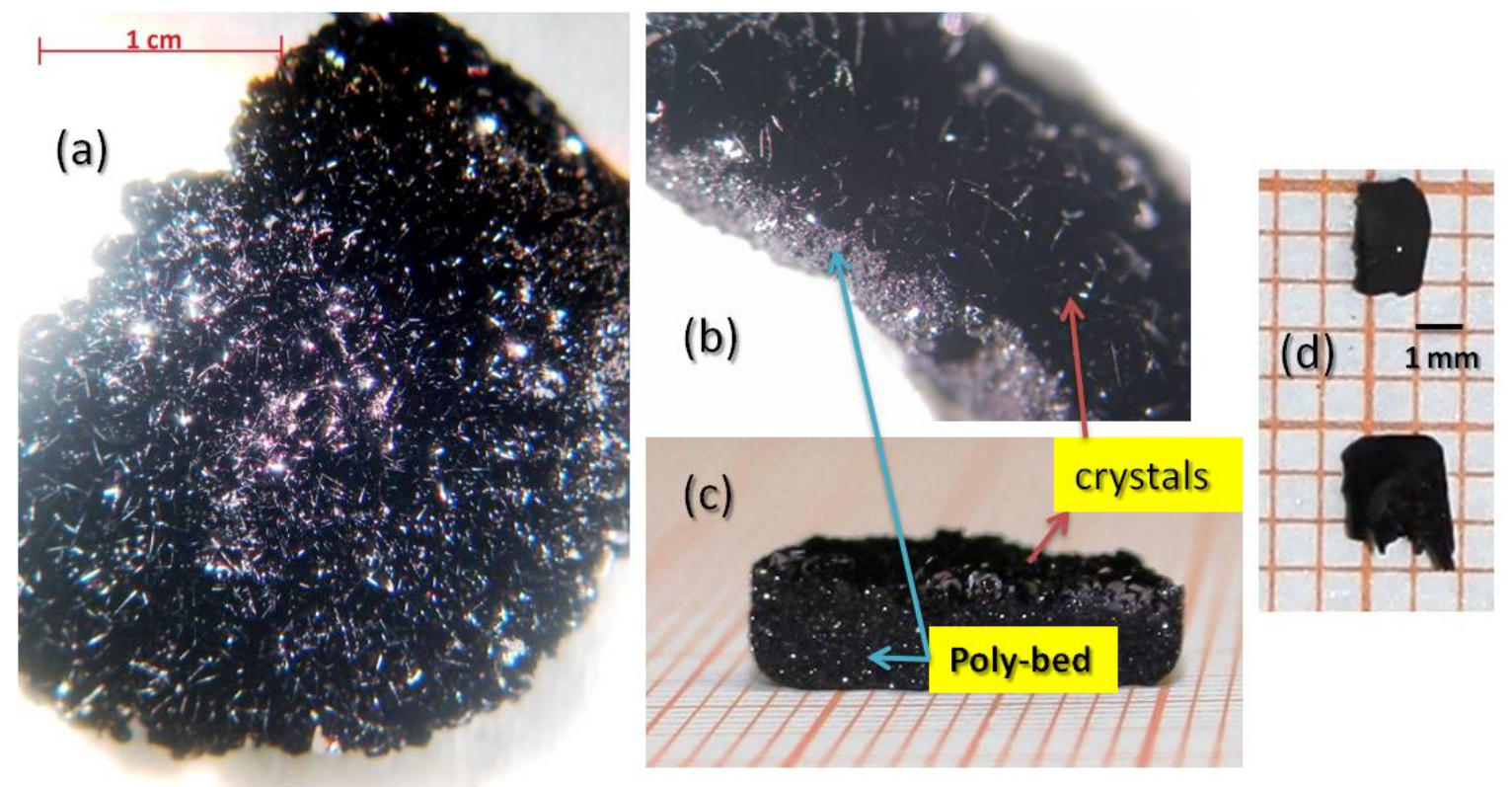

Figure 4.2: (a)View from the top of the $\mathrm{Na}_{2} \mathrm{IrO}_{3}$ crystal growth; (b) and (c) view from the side, focuses crystal bed above polycrystalline flux bed. (d) average size of the crystals after separation from polycrystalline bed.

\subsection{2 $\quad \mathrm{Na}_{2} \mathrm{IrO}_{3}$ single crystal growth}

In order to prepare $\mathrm{Na}_{2} \mathrm{IrO}_{3}$ single crystal, first $\mathrm{Na}_{2} \mathrm{IrO}_{3}$ polycrystal was prepared at $900^{\circ} \mathrm{C}$. Then $10 \%$ (atomic) $\mathrm{IrO}_{2}$ was added with it (even a extra heat treatment after adding $\mathrm{Ir}$ metal powder works the same) and homogeneously mixed using acetone. This homogeneous mixture of $90 \%$ (atomic) $\mathrm{Na}_{2} \mathrm{IrO}_{3}$ and $10 \% \mathrm{IrO}_{2}$ was put in a alumina crucible and was heated in open furnace at $1050^{\circ} \mathrm{C}$ for 72 hours. After furnace cooling to $900^{\circ} \mathrm{C}$, the crucible was air quenched. Cooling the furnace upto room temperature is avoided, as it may give some annealing effect to the crystals. It was observed that after such furnace cooling upto room temperature Na came out in the surface. Surface became less shiny with white patches, EDX measurement confirmed that those were the Na rich regions.

These crystals grow in an unusual different manner. Plate like crystals were vertically standing over a polycrystalline bed, randomly stacked and attached with one another in random angles between them [Fig. 5.1 (a)-(c)]. Each crystal was separated mechanically with sharp knife and sharp forceps. Average size of the crystal was $2 \mathrm{~mm} \times 1 \mathrm{~mm} \times 0.1 \mathrm{~mm}$ with $1 \mathrm{~g}$ of staring material in a $1 \mathrm{~cm}$ diameter crucible [Fig. 5.1(d)]. Size of the crystal depends upon size of crucible and amount of the polycrystalline material taken. Separating these crystals is very tricky as they are very thin and very randomly attached with one 
another. Lot of good crystals break but in a single attempt several crystals grow so getting enough crystals for measurement is not a problem, at least for undoped $\mathrm{Na}_{2} \mathrm{IrO}_{3}$.

In the crystal growth if that extra $10 \% \mathrm{IrO}_{2}$ is not added then height of the polycrystalline bed (poly-bed) increases and crystal dimension becomes smaller [Fig. 5.1 (b) and (c)]. This suggests that extra $\mathrm{IrO}_{2}$ is playing an important role in the crystal growth. It was also observed that very small $\mathrm{IrO}_{2}$ crystals grew on the inner wall and on the inner part of the lid of the crucible, possibly by $\mathrm{IrO}_{2}$ vapor transport. Melting point of $\mathrm{IrO}_{2}\left(1100^{\circ} \mathrm{C}\right)$ is higher than crystal growth temperature, melting point of $\mathrm{Na}_{2} \mathrm{IrO}_{3}$ is also much higher. Hence it is not a crystal growth from melt, it is not a solution growth like flux method. Random growth direction of the crystals over a polycrystalline bed suggests that it is Grain growth method where $\mathrm{Na}_{2} \mathrm{IrO}_{3}$ nucleated grains grow in size at the high temperature with time by the effect of $\mathrm{IrO}_{2}$ vapor pressure. $\mathrm{IrO}_{2}$ vapor pressure playing a crucial role in the crystal growth of the $\mathrm{Na}_{2} \mathrm{IrO}_{3}$.

\section{3 $\quad \mathrm{A}_{2} \mathrm{IrO}_{3}$ physical property}

Physical property of well ordered $\mathrm{Na}_{2} \mathrm{IrO}_{3}$ has been reported from our group by Yogesh Singh et. al. just before this thesis work[52]. Primarily, this thesis focus on the synthesize of phase pure $\mathrm{Li}_{2} \mathrm{IrO}_{3}$ system. The physical property of $\mathrm{Na}_{2} \mathrm{IrO}_{3}$ is also briefly discussed here as $\mathrm{Na}_{2} \mathrm{IrO}_{3}$ single and polycrystal was prepared for different experiments during this thesis. In the following section physical properties measured on well ordered single crystals and polycrystal of $\mathrm{Na}_{2} \mathrm{IrO}_{3}$ and $\mathrm{Li}_{2} \mathrm{IrO}_{3}$ are discussed.

\subsubsection{Structural characterization}

\subsubsection{X-Ray Diffraction of $\mathrm{Na}_{2} \mathrm{IrO}_{3}$ single crystals}

Powder x-ray diffraction (PXRD) of the ordered $\mathrm{A}_{2} \mathrm{IrO}_{3}$ polycrystal can be fitted to both $\mathrm{C} 2 / \mathrm{c}$ (space group no. 15) or $\mathrm{C} 2 / \mathrm{m}$ (space group no. 12 ) space group. $\mathrm{C} 2 / \mathrm{c}$ is basically supercell of $\mathrm{C} 2 / \mathrm{m}$ having double unit cell volume. Their lattice parameters are related in the following way: $\mathbf{a}^{\prime}=-\mathbf{a}, \mathbf{b}^{\prime}=-\mathbf{b}, \mathbf{c}^{\prime}=\mathbf{a}+2 \mathbf{c}, \mathrm{c}^{\prime}=\sqrt{ }\left(\mathrm{a}^{2}+4 \mathrm{c}^{2}+4 \mathrm{ac} \cos \beta\right), \sin \beta^{\prime}=\frac{2 c}{c^{\prime}}$ $\sin \beta\left(\operatorname{dashed}\left({ }^{\prime}\right)\right.$ are $\mathrm{C} 2 / \mathrm{c}$ lattice parameter and others are of $\left.\mathrm{C} 2 / \mathrm{m}\right)$. [36]

By the Rietveld analysis of $\mathrm{Na}_{2} \mathrm{IrO}_{3}$ PXRD data, it was predicted by Singh et.al. that $\mathrm{Na}_{2} \mathrm{IrO}_{3}$ crystallizes in $\mathrm{C} 2 / \mathrm{c}$ crystal structure and substantial amount of site exchange was 


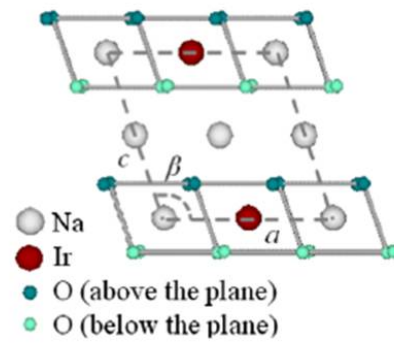

(a)

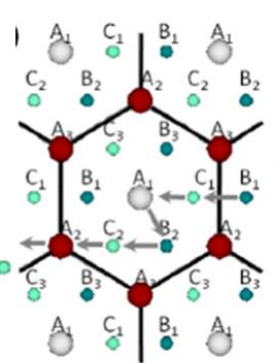

(b)

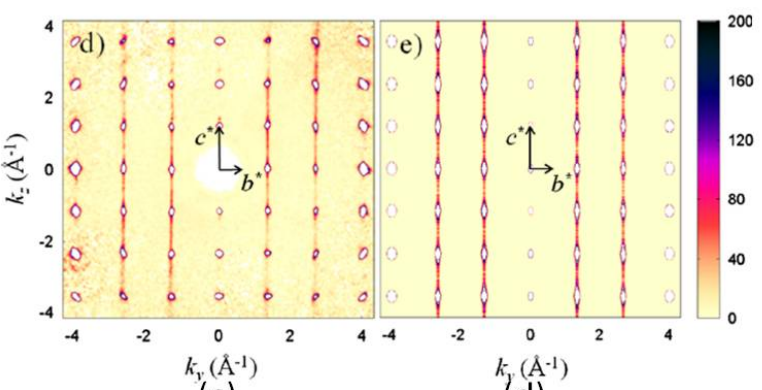

(c) (d)

Figure 4.3: (a) Monoclinic stacking along c-axis.(b) Illustration of layer stacking in the ideal honeycomb lattice and fault in stacking. (c) XRD intensity of (0kl) plane showing diffused rod indicating stacking fault, which is modeled in d) by frequent in-plane translational stacking faults of the type shown in b). Reprinted from [36].

considered.[52] But later Choi et. al. determined atomic strcuture of $\mathrm{Na}_{2} \mathrm{IrO}_{3}$ without any site mixing by single crystal XRD on the single crystals grown by Singh et. al.[36] After structural analysis with Rietveld analysis of the single crustal XRD and simulation using Vienna Ab-initio Simulation Package (VASP) they concluded C2/m is the ideal crystal structure of $\mathrm{Na}_{2} \mathrm{IrO}_{3}$, not $\mathrm{C} 2 / \mathrm{c}$. To prove that Choi et. al. gave following arguments:[36]

- As the $\mathrm{C} 2 / \mathrm{c}$ is supercell of $\mathrm{C} 2 / \mathrm{m}$, the single-crystal XRD data should show some superstructure peaks at (odd, odd, half integer), which was absent in the single crystal XRD data.

- Radial distribution function plot shows a considerable dispersion in Ir-Ir and Ir-O distances in the $\mathrm{C} 2$ /c model. Ir-Ir distance and Ir-O-Ir ranging from 1.99 to $2.43 \AA$ and 91 to $98^{\circ}$ in $\mathrm{C} 2 / \mathrm{c}$ model compared to 2.06 to $2.08 \AA$ and 98 to $99.4^{\circ}$ respectively in $\mathrm{C} 2 / \mathrm{m}$ model. It suggests that the $\mathrm{C} 2 / \mathrm{m}$ crystal structure is more symmetric.

- Structural optimization using VASP showed unusually high forces on oxygen in the $\mathrm{C} 2 / \mathrm{c}$ model exceeding over $3 \mathrm{eV} / \AA$ making $\mathrm{IrO}_{6}$ octahedra highly unstable in the $\mathrm{C} 2 / \mathrm{c}$ model.

Hence they totally discarded the $\mathrm{C} 2 / \mathrm{c}$ model for $\mathrm{Na}_{2} \mathrm{IrO}_{3}$ rather described $\mathrm{C} 2 / \mathrm{m}$ as the ideal stable crystal structure of $\mathrm{Na}_{2} \mathrm{IrO}_{3}$. Lattice parameters determined from Rietveld analysis

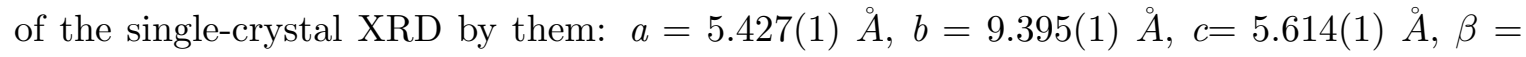
$109.037(18)^{\circ}$ and $Z=4 .[36]$

Another important result which came out from their structural analysis of $\mathrm{Na}_{2} \mathrm{IrO}_{3}$ was monoclinic stacking sequence of the honeycomb layers and the stacking fault in that sequence. Fig. 4.3(b) represents stacking sequence of the honeycomb layers after projecting 
all the layers in the basal plane. The stacking of Ir-O-Na-O-Ir layer is $\mathrm{A}_{1}$-B-C-A-B $-\mathrm{C}$. Same kind of stacking sequence was also reported in other layered (213) compounds.[56] This stacking sequence is achieved to minimize Coulomb energy between the layers. Due to weak interaction between layers faults occur in this sequence which is very clearly observed in single-crystal XRD. The diffused rod structure observed at the Bragg points of the $(0, k$, l) was due to stacking fault in the perpendicular direction of the stacking direction (in the Fig. 4.3(c) stacking fault is towards either $\mathrm{B}_{3}$ or $\mathrm{C}_{3}$ ). This was modelled by VASP which determined every $10^{\text {th }}$ honeycomb layer undergoes such a fault[Fig. 4.3(d)]. But such a small amount of stacking fault does not disturb long range ordering of the crystal and magnetic structure.[36]

Other important things which was reported from single crystal XRD of $\mathrm{Na}_{2} \mathrm{IrO}_{3}$ by Ye et. al. were $\mathrm{IrO}_{6}$ octahedron is under trigonal distortion, O-Ir-O angle perpendicular to the basal plane are greater than $90^{\circ}$ and angles across the shared edges are less than $90^{\circ}$, Ir-O-Ir is between 98 to $99.4^{\circ}$ [57]. For two undistorted edge sharing octahedra, this angle should be exactly $90^{\circ}$ which results ideal anisotropic Kitaev interaction without any isotropic contribution.

\subsubsection{X-Ray Diffraction of $\mathrm{Li}_{2} \mathrm{IrO}_{3}$ polycrystal}

It is very hard to distinguish between $\mathrm{C} 2 / \mathrm{c}$ and $\mathrm{C} 2 / \mathrm{m}$ model from the fitting quality of PXRD of $\mathrm{Na}_{2} \mathrm{IrO}_{3}$. But for $\mathrm{Li}_{2} \mathrm{IrO}_{3}$ it was clearly seen that $\mathrm{C} 2 / \mathrm{m}$ gives better fitting of PXRD pattern on well ordered polycrystal. Earlier Kobayashi et. al. [54] described $\mathrm{Li}_{2} \mathrm{IrO}_{3}$ both by $\mathrm{C} 2 / \mathrm{c}$ and $\mathrm{C} 2 / \mathrm{m}$ model but later O'Malley et. al [55] described $\mathrm{Li}_{2} \mathrm{IrO}_{3}$ by the $\mathrm{C} 2 / \mathrm{m}$ crystal structure and they had same observation like us.

Powder XRD pattern of $\mathrm{Li}_{2} \mathrm{IrO}_{3}$ produced by $\mathrm{LiCl}$ flux is shown Fig. 4.4. All the lines $\left(\mathrm{I}_{o b s}\right)$ of the PXRD pattern between $2 \theta=10$ to $100^{\circ}$ are well indexed by $\mathrm{C} 2 / \mathrm{m}$ crystal structure. Absence of any unidexed line confirms removal of all $\mathrm{LiCl}$ flux from the final $\mathrm{Li}_{2} \mathrm{IrO}_{3}$ polycrystalline batch. PXRD pattern was theoretically fitted by Rietveld analysis using GSAS ( $\mathrm{I}_{c a l}$ in Fig. 4.4).[46, 47] Difference curve $\left(\mathrm{I}_{d i f f}=\mathrm{I}_{o b s}-\mathrm{I}_{c a l}\right)$ is also shown in the graph. Table 4.1 lists all the structural parameters for the Rietveld analysis. Lattice parameters from Rietveld analysis are $\mathrm{a}=5.1695(5) \AA \mathrm{A}, \mathrm{b}=8.9371(8) \AA, \mathrm{c}=5.1226(4) \AA$ $109.800(7)^{\circ}$ and volume is $222.673(27) \AA^{3}$. Volume is significantly decreased in $\mathrm{Li}_{2} \mathrm{IrO}_{3}$ compared toNa $\mathrm{IrO}_{3}$ also $c / a$ ratio is decreased by more than $5 \%$ which implies that going from Na-system to Li-system is equivalent to an effective c-axis pressure. Ir-Ir distances in 


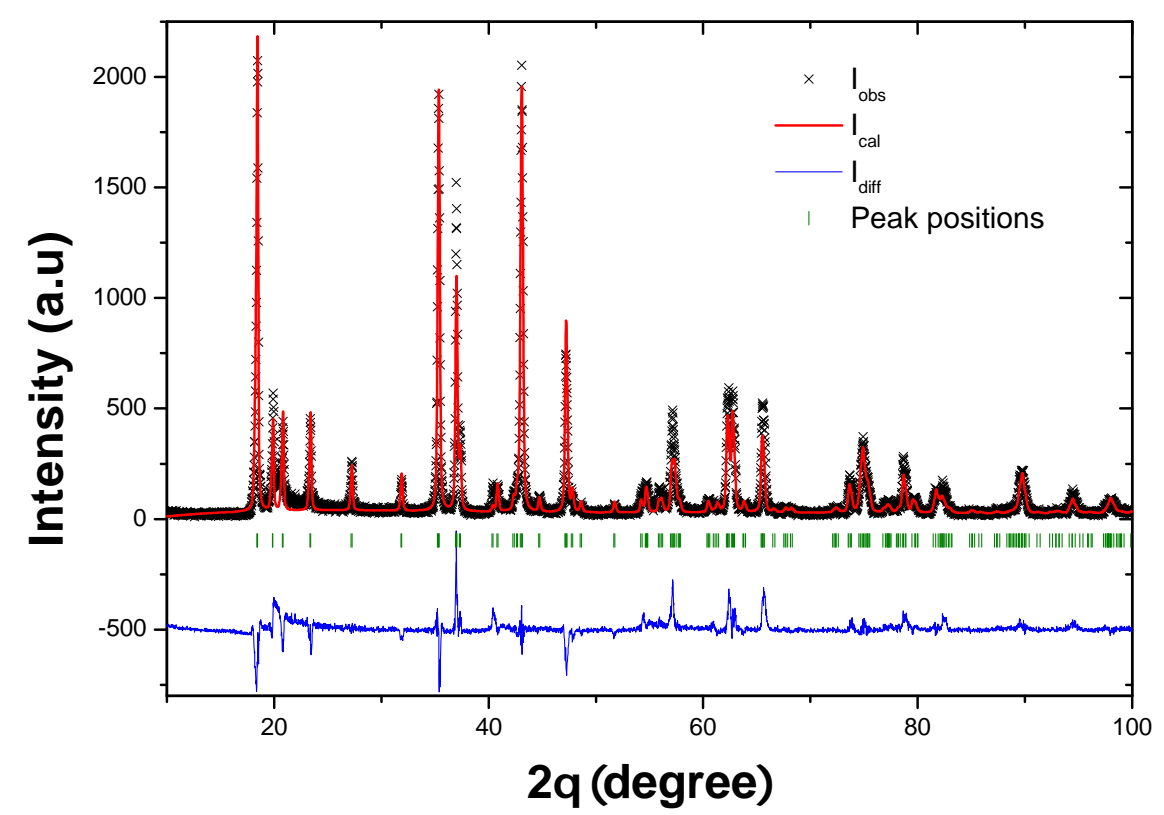

Figure 4.4: $\mathrm{PXRD}$ of $\mathrm{Li}_{2} \mathrm{IrO}_{3}$ prepared by $\mathrm{LiCl}$ flux (X marks), Theoretical fitting after Rietveld analysis (red line), difference curve of the theoretical and experimental data (blue line) with peak positions marked.

Table 4.1: Structural parameters of $\mathrm{Li}_{2} \mathrm{IrO}_{3}$ obtained for Rietveld refinements of powder $\mathrm{XRD}$ pattern by $\mathrm{C} 2 / \mathrm{m}$ space group (no.12). Fitting quality: $\mathrm{Chi}^{2}=5.493, \mathrm{R}_{w p}=0.253$, $\mathrm{R}_{p}=0.202$.

\begin{tabular}{ll|ccccc}
\hline atom & position & $\mathrm{x}$ & $\mathrm{y}$ & $\mathrm{z}$ & Occ. & $\mathrm{B}$ \\
\hline \hline $\mathrm{Ir}$ & $4 \mathrm{~h}$ & 0 & $0.33201(33)$ & 0 & 0.905 & 0.01 \\
$\mathrm{Li}$ & & 0 & $0.33201(33)$ & 0 & 0.095 & 0.02 \\
\hline $\mathrm{Li}$ & $2 \mathrm{a}$ & 0 & 0 & 0 & $0.791(5)$ & 0.02 \\
$\mathrm{Ir}$ & & 0 & 0 & 0 & $0.209(5)$ & 0.01 \\
\hline $\mathrm{Li}$ & $4 \mathrm{~g}$ & 0 & $0.256(8)$ & 0.5 & 1 & 0.02 \\
$\mathrm{Li}$ & $2 \mathrm{c}$ & 0 & 0.5 & 0.5 & 1 & 0.02 \\
$\mathrm{O}$ & $8 \mathrm{j}$ & $0.264(7)$ & $0.3207(26)$ & $0.6979(33)$ & 1 & 0.03 \\
$\mathrm{O}$ & $4 \mathrm{i}$ & $0.220(8)$ & 0 & $0.209(5)$ & 1 & 0.03 \\
\hline
\end{tabular}

$\mathrm{Li}_{2} \mathrm{IrO}_{3}$ are 2.9715(5) and 3.003(8) $\AA$ which is shorter than the one in Na-system. $\mathrm{IrO}_{6}$ octahedra become more symmetric compared to $\mathrm{Na}_{2} \mathrm{IrO}_{3}$ as Ir-O distances ranges from 2.290 to $2.386 \stackrel{\AA}{A}$ in $\mathrm{Li}_{2} \mathrm{IrO}_{3}$. Fig. 4.4 shows Rietveld fitting is not very good in the range of $2 \theta=19$ to $33^{\circ}$ it has some continuous slope in the difference curve $\left(\mathrm{I}_{\text {diff }}\right)$. This effect is incorporated in fitting by partial site mixing between Ir position (4h) and Li position at the center of the honeycomb (2a) but this slope comes from the stacking fault in the honeycomb layers. Rietveld method does not have better mechanism to account stacking fault so a bad fitting is obtained in that region. This is discussed in Disorder in $\mathrm{A}_{2} \mathrm{IrO}_{3}$ section in more detail. 


\subsubsection{Pair Distribution Function (PDF) Analysis of $\mathrm{A}_{2} \mathrm{IrO}_{3}$}

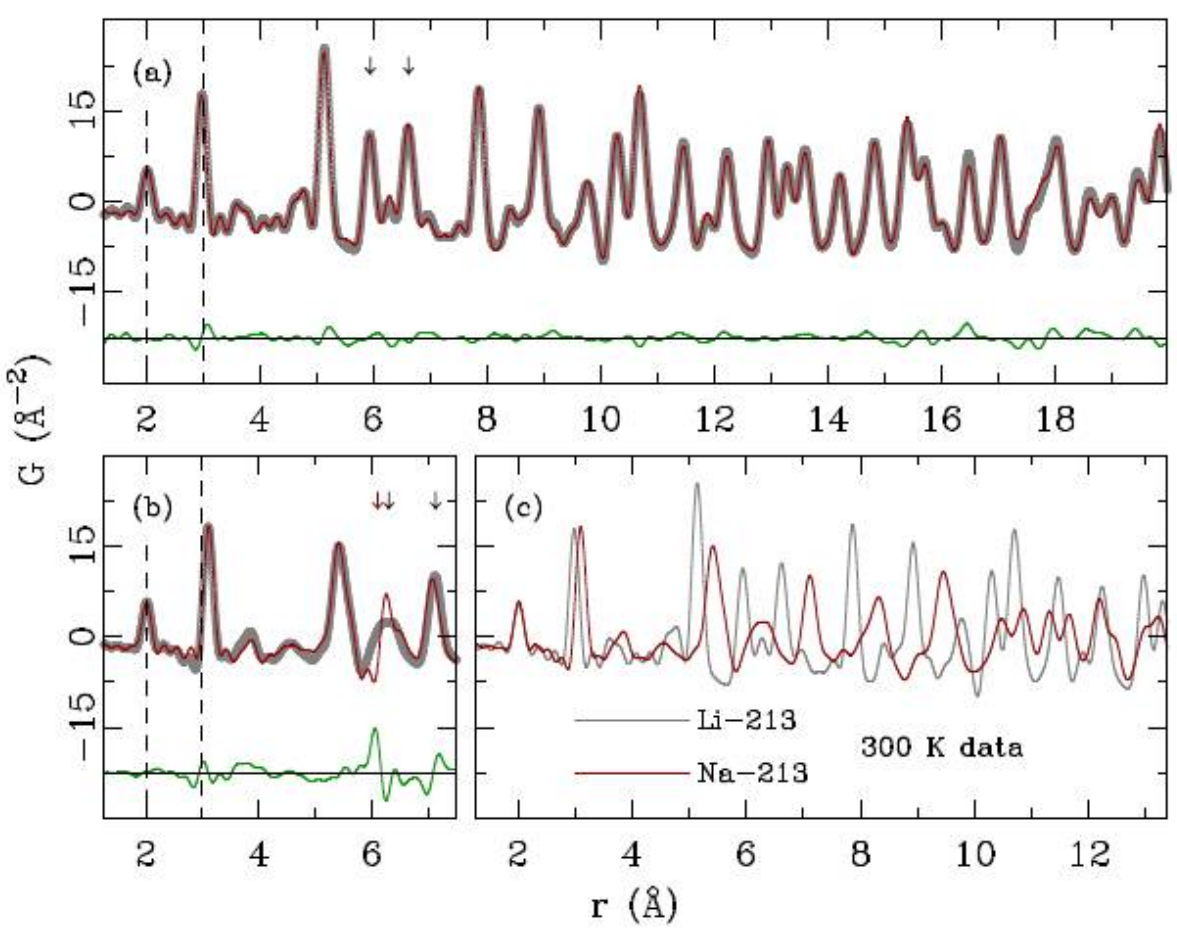

Figure 4.5: Atomic PDF of $\mathrm{Li}_{2} \mathrm{IrO}_{3}(\mathrm{a})$ and $\mathrm{Na}_{2} \mathrm{IrO}_{3}(\mathrm{~b})$, grey symbols experimental data, red lines are refinement by $\mathrm{C} 2 / \mathrm{m}$ model and green is the difference curve. (c) Direct comparison of the both system PDF data measured at 300K. Data was measured by H. Gretarsson et.al. on our produced sample, reprinted from[58].

Powder XRD measurement and Rietveld analysis has many shortcomings to analyze local structure and local distortions. It is also not very efficient to determine beyond next neighbor atomic correlation. For more detail analysis of the local structural information a comparative study was done on $\mathrm{Li}_{2} \mathrm{IrO}_{3}$ polycrystal and $\mathrm{Na}_{2} \mathrm{IrO}_{3}$ single crystal by pair distribution functional (PDF) analysis of total x-ray scattering measured in a synchrotron source by our collaborator H. Gretarsson et. al. and reported in Ref. [58].

Total scattering treats sharp Bragg scattering as well as diffuse scattering from long range atomic order and local distortions. In the PDF analysis coherent scattering intensity $I(Q)$ is measured in reciprocal Q-spacae over a huge range upto almost $30-50 \AA^{-1}$. And maximum $\mathrm{Q}$ attainable in back scattering of $\mathrm{Cu}-\mathrm{K}_{\alpha}$ source is around $8 \AA^{-1}$. Usually for highest real-space resolution and smallest statistical uncertainty synchrotron data are used for PDF analysis. Now the Q-space intensity $I(Q)$ is normalized by dividing square of atomic form factor to obtain the reduced total-scattering structure function $F(Q)$, where $\mathrm{F}(\mathrm{Q})=\mathrm{Q}[\mathrm{S}(\mathrm{Q})-1], S(Q)$ is the total-scattering structure function. This reciprocal space 
function $F(Q)$ is transformed into real-space function pair distribution function $G(r)$ by Fourier transformation. [59]

In the Fig. 4.5 PDF data of $\mathrm{Na}_{2} \mathrm{IrO}_{3}$ and $\mathrm{Li}_{2} \mathrm{IrO}_{3}$ is shown which were measured by Greatarsson et. al. on the samples prepared in our lab.[58] First time such a measurement gave a deeper insight of structural details of $\mathrm{Li}_{2} \mathrm{IrO}_{3}$ in spite of no single crystal. $\mathrm{Li}_{2} \mathrm{IrO}_{3} \mathrm{PDF}$ data was fitted with $\mathrm{C} 2 / \mathrm{m}$ model using PDFgui which gave all the structural parameters including all detail atomic positions (Fig. 4.5.a). O position were refined with better accuracy than Rietveld analysis of PXRD data. This resulted in little less value of lattice parameters compared to value obtained by Rietveld analysis of the PXRD data $(<0.1 \%$ change $)$. First peak observed at around $\mathrm{r}=2 \AA$ is same for $\mathrm{Li}_{2} \mathrm{IrO}_{3}$ and $\mathrm{Na}_{2} \mathrm{IrO}_{3}$ represents Ir-O bond length which varies between 2.01 to $2.04 \AA$. Ir-Ir distance and Ir-O-Ir bond angel ranges from 2.984 to $2.992 \AA$ and 93.6 to $94.8^{\circ}$ for $\mathrm{Li}_{2} \mathrm{IrO}_{3}$. Ir-Ir distance reduced in $\mathrm{Li}$ system and also IrO-Ir bond distance is more near to $90^{\circ}$ compared to Na-sysetm. Somehow $\mathrm{Na}_{2} \mathrm{IrO}_{3} \mathrm{PDF}$ data was not that sharp like $\mathrm{Li}_{2} \mathrm{IrO}_{3}$ (Fig. 4.5.b,c) so it was not possible to resolve all the structural parameters from here.[58] This may be originated from structural fault and/or due to introducing further disorder by crushing of the crystals to make it powder.

Hence a structural insight of $\mathrm{Li}_{2} \mathrm{IrO}_{3}$ by PDF analysis concludes that here Ir-honeycomb is more reduced in size which increased beyond next neighbor atomic correlation between Ir atoms and also $\mathrm{IrO}_{6}$ octahedra become more symmetric compared to Na-system. Hence Li-system nearer to ideal Kitaev bond geometry which should be reflected into the magnetic property.

\subsubsection{Resistivity}

Fig 4.6(a) shows in plane resistivity $\rho(T)$ measured on $\mathrm{Na}_{2} \mathrm{IrO}_{3}$ single crystals [52] and Fig 4.6(b) shows bulk resistivity $\rho(T)$ of $\mathrm{Li}_{2} \mathrm{IrO}_{3}$ polycrsystal, measured on a cuboid shape piece of highly pressurized pellet. Temperature dependence shows both $\mathrm{Na}_{2} \mathrm{IrO}_{3}$ and $\mathrm{Li}_{2} \mathrm{IrO}_{3}$ are insulators. Earlier $\mathrm{Li}_{2} \mathrm{IrO}_{3}$ had been reported as a metallic conductor from the conductivity measurement in the temperature range 400 - $1000 \mathrm{~K}$. [55] It is clear that above $200 \mathrm{~K}$ increase in $\rho(T)$ is rather small. This possibly can be the reason, why conductivity measurement at higher temperature by O'Malley et. al. did not see any change. First time $\mathrm{Li}_{2} \mathrm{IrO}_{3}$ was reported an insulator by us.[60]. A strong logarithmic increase of $\rho(T)$ at low $T$ was observed for both $\mathrm{Na}_{2} \mathrm{IrO}_{3}$ and $\mathrm{Li}_{2} \mathrm{IrO}_{3}$. 

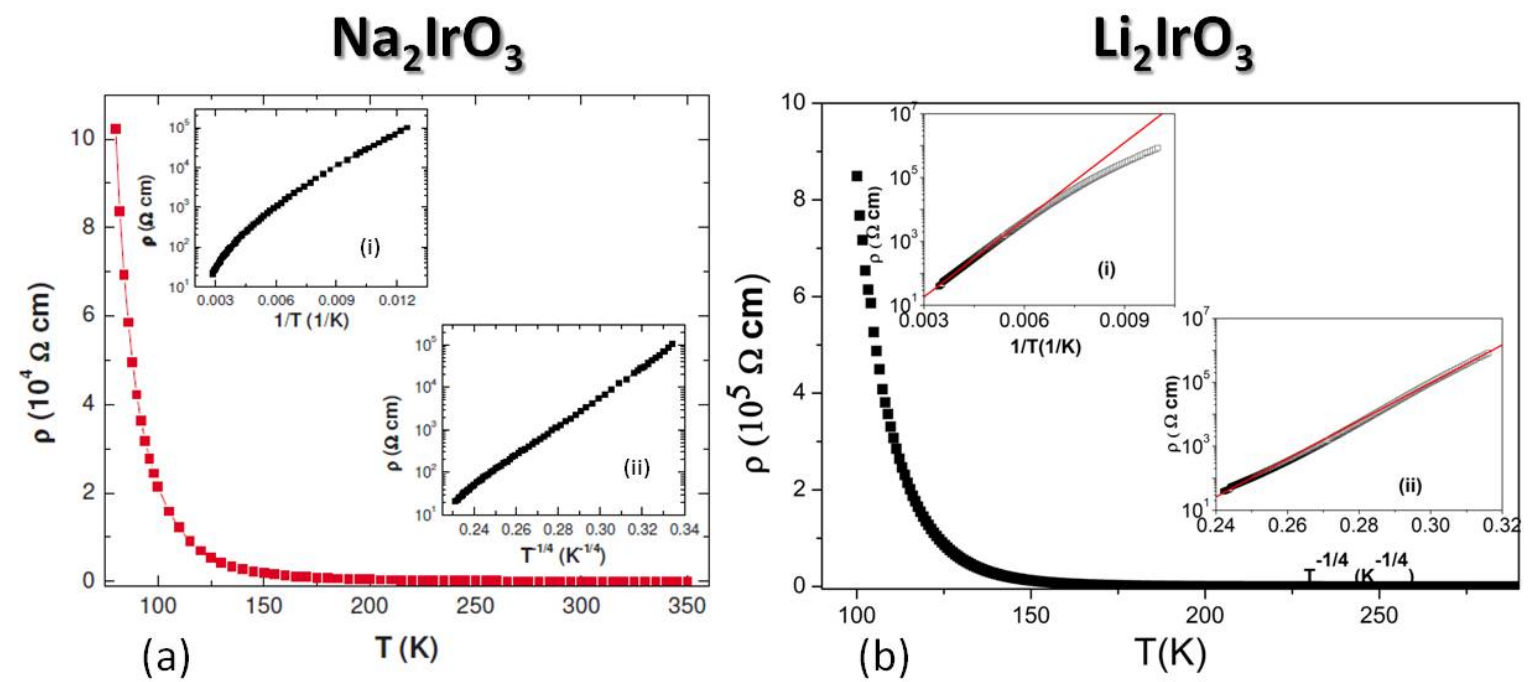

Figure 4.6: (a) In plane resistivity $\rho(T)$ of $\mathrm{Na}_{2} \mathrm{IrO}_{3}$ single crystals measurement by Yogesh Singh et.al, reprinted from [52]. (b) Bulk resistivity $\rho(T)$ of the $\mathrm{Li}_{2} \mathrm{IrO}_{3}$ highly pressurized polycrystal. In both the insets $\mathrm{i}$, ii of a and b show fitting of the $\rho(T)$ by Arrhenius behavior and variable range hopping (VHR) behavior respectively.

Resistivity of many band insulators and semiconductors follow activation behavior given by Arrhenius law:

$$
\rho(T) \propto \exp (-\Delta E / T)
$$

But inset (i) of the Fig. $4.6 \mathrm{a}$ and $\mathrm{b}$ show both of the systems deviate from the this activation behavior (Eqn. 4.3). For $\mathrm{Li}_{2} \mathrm{IrO}_{3}$ polycrystal rather it clearly shows two different slopes at low and high temperature. Arrhenius behavior can be fitted in the high temperature range (200 -300K) which gives activation gap of $160 \mathrm{meV}$ for $\mathrm{Li}_{2} \mathrm{IrO}_{3}$ polycrystal. For $\mathrm{Na}_{2} \mathrm{IrO}_{3}$, it is more than $350 \mathrm{meV}$. This value does not represent charge gap which was rather precisely determined from optical conductivity measurement. Value of the charge gap obtained from the optical conductivity measurement on $\mathrm{Na}_{2} \mathrm{IrO}_{3}$ single crystal was $340 \mathrm{meV}$ [61]. Insets (ii) of Fig 4.6 shows that $\rho(T)$ behavior of $\mathrm{Na}$ and Li-system rather follows 3D variable range hopping(VHR) behavior given by:

$$
\rho(T) \propto \exp \left[\left(T_{\circ} / T\right)^{n}\right]
$$

Where $n=1 /(d+1)$. For $3 \mathrm{D}(\mathrm{d}=3)$ VHR behavior $n=1 / 4$ and the VHR relation is given by:

$$
\rho(T)=\rho_{\circ} \exp \left[\left(T_{\circ} / T\right)^{1 / 4}\right]
$$

In the inset (ii) of the Fig. 4.6 (a) and (b), $\log \rho(T)$ versus $T^{-1 / 4}$ plot for $\mathrm{Na}_{2} \mathrm{IrO}_{3}$ and $\mathrm{Li}_{2} \mathrm{IrO}_{3}$ show a linear behavior and a very little curvature is observed at high $T$, but low $T$ 
data can be well fitted with VHR behavior (Eqn. 4.5) which gives $T_{\circ} \approx 10^{7} \mathrm{~K}$ and $\rho_{\circ} \approx 10^{-4}$ $\Omega$-cm . Deviation of the VHR behavior at higher $T$ also has been observed in the other spinorbit Mott insulator $\mathrm{Sr}_{2} \mathrm{IrO}_{4}$ also. [62, 63]. There VHR behavior was fitted for polycrystalline $\mathrm{Sr}_{2} \mathrm{IrO}_{4}$ at the $T$-range 30 to $100 \mathrm{~K}$. For the $\mathrm{Li}_{2} \mathrm{IrO}_{3}$ polycrystal, VHR behavior is fitted in between 90 to $150 \mathrm{~K}$ (measurement at the lower temperature was not possible by four probe setup as maximum value of the resistance $R$ was reached after strong increase at low temperature). Above $150 \mathrm{~K}$ Arrhenius behavior is fitted. This crossover from Arrhenius behavior to VHR behavior was observed in other localized insulating systems also. [62, 64]

VHR mechanism of electron conduction was first formulated by Mott. It was assumed for a localized system hopping probability of a electron is given by :

$$
P=P_{\circ} \exp \left(-2 r / a-\Delta E / k_{B} T\right)
$$

where $r$ and $\Delta E$ are spatial distance and energy difference between the two hopping state respectively, $a$ is the electron localization length, $P_{\circ}$ is a constant and $\mathrm{k}_{B}$ is the Boltzman's constant. One can also understand this crossover from Arrhenius behavior to VHR behavior empirically from Eqn. 4.6. A hopping of electron will always try to find the lowest activation energy $\Delta E$ and the shortest hopping distance $r$. However, usually these two conditions cannot be satisfied at the same time. Thus there will be an optimum hopping distance $r$, which maximizes the hopping probability $P$.[65] At high temperature hopping is dominated by nearest neighbor hopping where $r$ is very small and $\Delta E$ is very high $\left(\propto 1 / \mathrm{r}^{3}\right)$. Hence in Eqn. $4.61^{\text {st }}$ term within exponential becomes negligible and it leads to a activated Arrhenius behavior of resistivity (Eqn. 4.3) and conduction is dominated by thermally activated mechanism. When the temperature decreases, number of states available for thermally activated hopping decrease. Then hopping has to be through low energy states (near to Fermi energy) which are spatially highly separated (i.e. $r$ is large). This states are basically impurity sates coming from disorder, situated near to the Fermi energy. Now conduction happen through the hopping between these impurity sates of variable range $(r)$ for which Mott derived VHR behavior of resistivity by taking $\Delta E \propto 1 / \mathrm{g}_{\circ} \mathrm{r}^{n}$, where $n$ is dimensionality of hopping and $g_{\circ}$ is the constant density of states at the Fermi level.[66]

\section{Electrical anisotropy in $\mathrm{Na}_{2} \mathrm{IrO}_{3}$ :}

Crystal structure of $\mathrm{A}_{2} \mathrm{IrO}_{3}$ suggests that it is a very layered structure, stacked along c-axis. So for such a quasi $2 \mathrm{D}$ system anisotropy in different physical property is quite expected. Here anisotropy in the resistivity has been investigated on the $\mathrm{Na}_{2} \mathrm{IrO}_{3}$ single crystals. They are very plate like crystals and the Laue diffraction pattern suggests that 


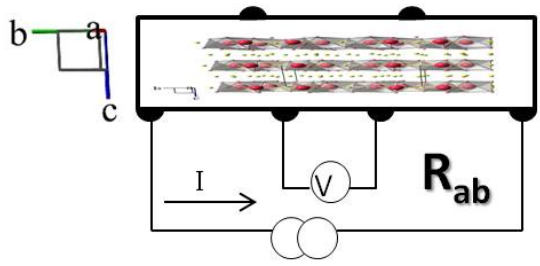

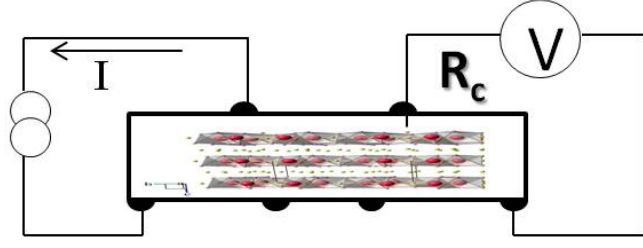

(a)

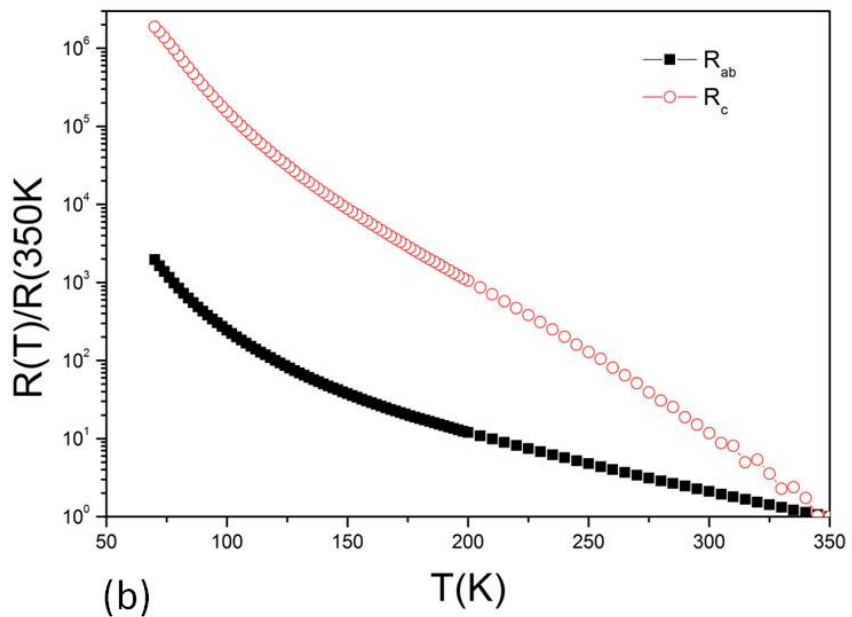

(b)

Figure 4.7: (a) Schematic diagram of two resistivity measurement configuration in ab plane $\left(\mathrm{R}_{a b}\right)$ and perpendicular to ab plane along c axis $\left(\mathrm{R}_{c}\right)$. (b) Normalized resistivity $\mathrm{R}_{a b}$ and $\mathrm{R}_{c}$ measured for $\mathrm{Na}_{2} \mathrm{IrO}_{3}$.

planes of those thin plates are $a b$-plane of the crystal structure and perpendicular to that plate is c-axis. So resistivity was measured in two configuration on those thin plate like crystals. First, all the four contacts on the one face of the thin plate which gives resistivity of the ab plane mainly $\left(\mathrm{R}_{a b}\right)$ [Fig4.7(a)]. Second, two voltage contacts on the two opposite side of the plate and two current contacts also on two opposite side of the plate [Fig4.7(a)]. In this configuration current passes from one side to the other of the plate that is along c-axis and measures voltage along c-axis, which gives $\mathrm{R}_{c}$. In the Fig. 4.7(b) normalized resistance $R(T) / R(350 K)$ is plotted for both the configurations. Value of $R(T) / R(350 K)$ along c-axis is 3 order of magnitude higher than along ab-plane at $75 \mathrm{~K}$ (lowest temperature measured). Such a anisotropic result confirms low dimensionality of the structure. Now in this system current flows due to hopping of charge carrier between localized states around Fermi level. Near the Fermi level electronic states are dominated Ir 5d states so this hopping will be much higher in $a b$-plane where Ir-honeycomb is situated, rather than along $c$-axis. Also due to higher packing in the $a b$-plane hopping of charge carrier is more probable than the along c-axis.

\subsubsection{Magnetization}

\subsubsection{1 $\quad \mathrm{Na}_{2} \mathrm{IrO}_{3}$}

Yogesh Singh et. al. first reported long range antiferromagnetic (AF) ordering below $T_{N}=$ $15 \mathrm{~K}$ in $\mathrm{Na}_{2} \mathrm{IrO}_{3}$, measured on single crystals.[52]. Same result was reproduced in this thesis. 


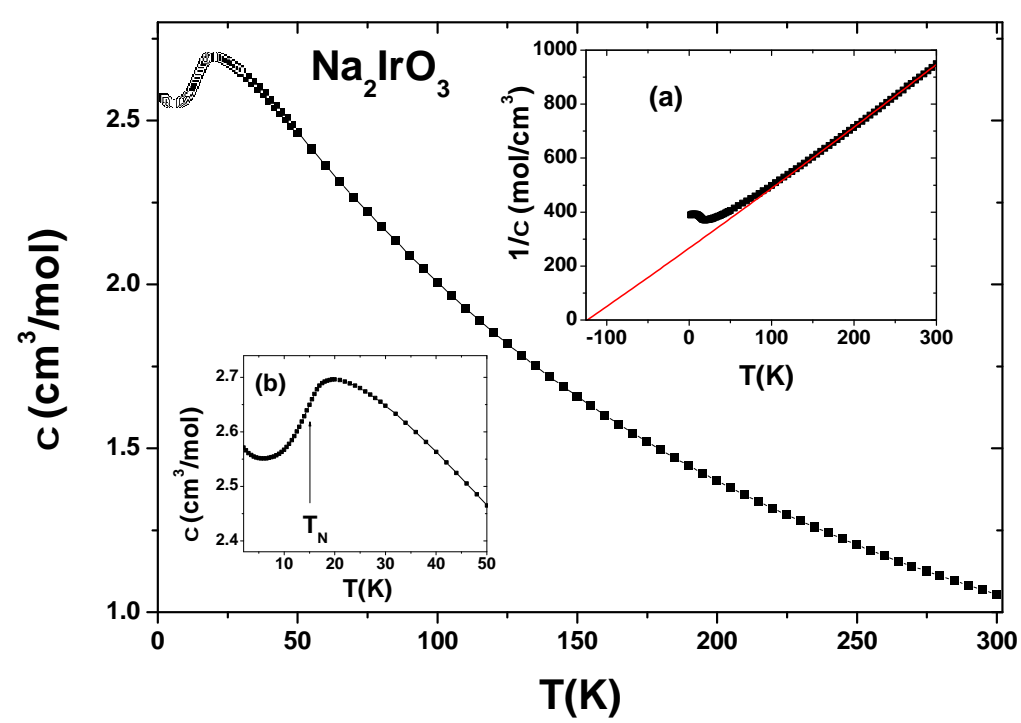

Figure 4.8: Magnetic susceptibility $\chi(T)$ versus temperature $T$ for $\mathrm{Na}_{2} \mathrm{IrO}_{3}$. Inset a, shows $\mathrm{CW}$ fitting of $1 / \chi$ versus $T$ data. Inset $\mathrm{b}$, closer view of the drop in $\chi$ at low temperature, vertical arrow points to $\mathrm{AF}$ ordering temperature $T_{N}$.

Fig. 4.8 shows magnetic susceptibility $\chi(T)$ versus temperature $T$ measured on $\mathrm{Na}_{2} \mathrm{IrO}_{3}$ wellordered polycrystal. A broad hump is observed at low $T$ around $T=23 \mathrm{~K}$ onset $T_{N}=15 \mathrm{~K}$ (inset b). This broad hump is usually observed in antiferromagnetic low dimensional system where short range order develops well above $T_{N}[52,67]$ and 3D magnetic ordering observed when inter-planar interactions become important. Also in these low dimensional systems 3D ordering between all the magnetic layers(here honeycomb layers) happen in a broad temperature range compared to three dimensional magnetic system. Hence susceptibility drops more abruptly at $\mathrm{T}_{N}$ in the three dimensional system than the low dimensional systems where susceptibility drops after a broad hump.

High temperature susceptibility follows Curie-Weiss (CW) behavior:

$$
\chi=\chi_{0}+\frac{C}{T-\theta_{W}}
$$

The CW fit (Eqn. 4.7) of the $1 / \chi\left(T\right.$ versus $T$ data gives $C=0.42 \mathrm{~cm}^{3} \mathrm{~K} / \mathrm{mol}, \theta_{W}=-$ $120(20) \mathrm{K}$ (inset a) $\chi_{0}=-5.2(5) \times 10^{-5} \mathrm{~cm}^{3} / \mathrm{mol}$. This value of $C$ corresponds to the effective moment $\mu_{e f f}=1.83 \mu_{B}$, which is close to value $1.74 \mu_{B}$ expected for $\mathrm{S}=1 / 2$ moment. High -ve CW temperature $\theta_{W}$ signifies strong AFM exchange interaction between Ir $\mathrm{S}_{\text {eff }}$ $=1 / 2$ moments. Small $\chi_{0}$ is coming from either Van Vlec paramagnetism or from background of the measurement. All the value obtained here are very much comparable to the earlier report by Yogesh Singh et. al. 


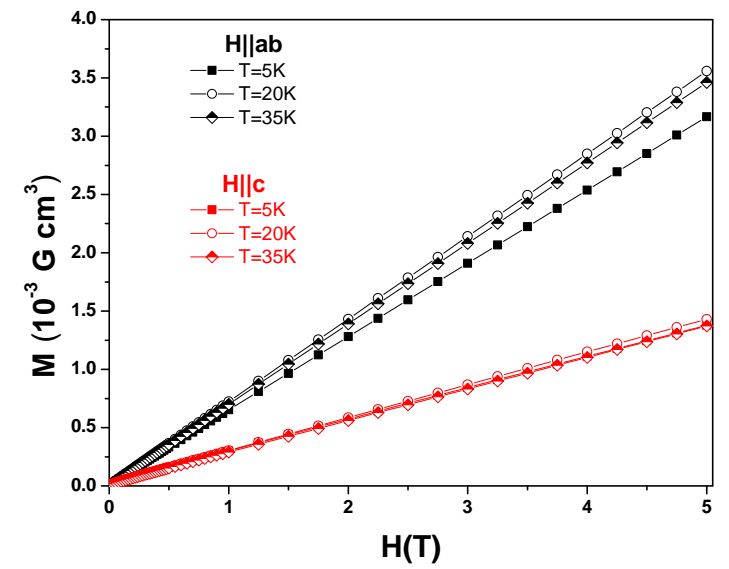

Figure 4.9: Anisotropy in Magnetization $M$ versus $H$ for $\mathrm{Na}_{2} \mathrm{IrO}_{3}$ at different $T$ for field along ab-palne and along c-axis. It was measured in same configuration as reported by Singh et. al.[52]

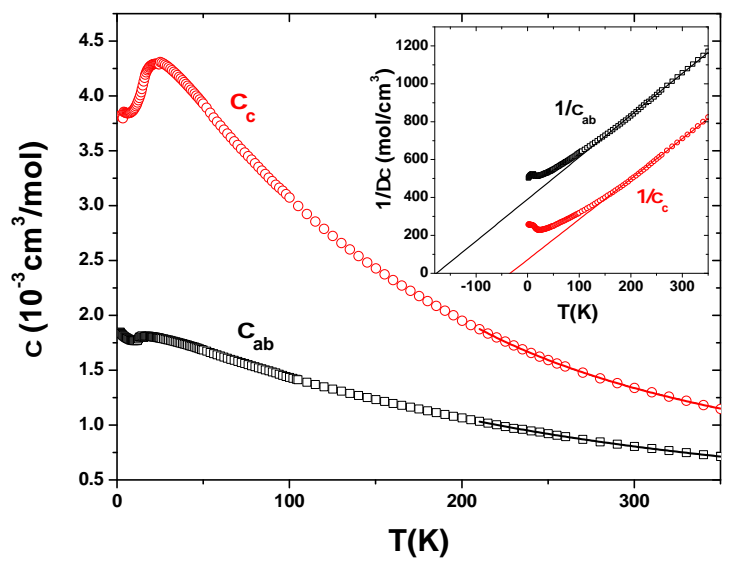

Figure 4.10: Anisotropy in susceptibility versus temperature for field along abplane and c-axis in $\mathrm{Na}_{2} \mathrm{IrO}_{3}$, (measured by Singh et.al[52] and re-plotted for further analysis with the original data file). (inset) $\mathrm{CW}$ fit of $1 / \Delta \chi$ versus $T$ data for respective Weiss temperature $\theta_{W}$ (represented by intersect at $T$-axis.

\section{Magnetic anisotropy in $\mathrm{Na}_{2} \mathrm{IrO}_{3}$ :}

Yogesh Singh et. al. have already investigated the magnetic anisotropy in $\mathrm{Na}_{2} \mathrm{IrO}_{3}$. [52] . They reported $\frac{\chi_{a b}}{\chi_{c}}=1.5$ and 2.3 at $\mathrm{T}=400 \mathrm{~K}$ and $1.8 \mathrm{~K}$ respectively, where $\chi_{c}$ and $\chi_{a b}$ are the magnetic susceptibilities measured with field applied along c-axis and perpendicular to c-axis(along ab-plane) respectively, suggesting a weekly anisotropic magnetic system. Over the whole temperature $(T)$ range $\chi_{a b}>\chi_{c}$. The origin of this anisotropy was interpreted from g-factor anisotropy which they obtained fitting CW law in $\chi_{a b}$ and $\chi_{c}$ with same $\theta_{W}$ and they got $\mathrm{g}_{c}$ and $\mathrm{g}_{a b} 2.68$ and 1.87 respectively. Due to presence of trigonal distortion in the $\mathrm{IrO}_{6}$ octahedra a small g-factor anisotropy is expected though $g_{a b}$ obtained from electron spin resonance (ESR) is close to 2.[68] This indicates that the origin of the anisotropy could be something other than only a single-ion anisotropy .

To find out the origin of this anisotropy, magnetization $(M)$ versus applied magnetic field $(H)$ was measured for the field applied along ab-plane and $c$-axis, ${ }^{1}$ in the same way it was reported by Singh et. al. [52] Fig. 4.9 shows that for the all the temperature range magnetization has linear behavior with varying magnetic field. Hence, there is no dependence of this anisotropy on low magnetic field (till 5T) and no signature of any meta-magnetic transition. Then the the data of $\chi_{a b}$ and $\chi_{c}$ measured by Yogesh Singh was re-analyzed by CW fitting (Fig. 4.10). From the CW fitting (Eqn.4.7) of $\chi_{a b}$ and $\chi_{c}$ versus $T$ data in the

\footnotetext{
${ }^{1}$ This measurement was done in the measurement configuration prepared by Yogesh Singh
} 


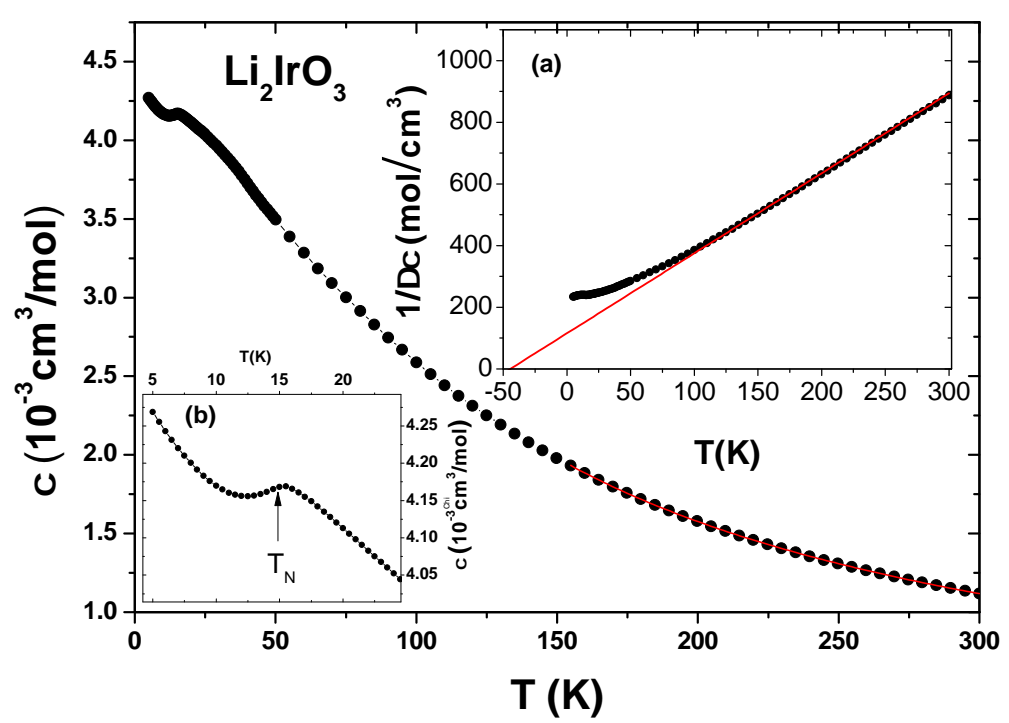

Figure 4.11: Magnetic susceptibility $\chi=M / H$ versus temperature $T$ for $\mathrm{Li}_{2} \mathrm{IrO}_{3}$, red line signifies fit by $\mathrm{CW}$ law.(inset, a) $\mathrm{CW}$ fitting of $1 / \Delta \chi$ versus $T$ data for Weiss temperature and effective moment $\left(\Delta \chi=\chi-\chi_{0}, \chi_{0}\right.$ from $\mathrm{CW}$ fit of $\chi$ vs. $T$ data at higher value). (inset, b) Closer view to the drop in $\chi$ at low temperature, vertical arrow points to AF ordering temperature $T_{N}$.

range 200-350K (solid lines) $\chi_{0}$ was obtained for the respective measurements which were almost similar. Then that $\chi_{0}$ was subtracted from respective $\chi(T)$ to obtain $\Delta \chi\left(=\chi-\chi_{0}\right)$. In the inset $1 / \Delta \chi$ versus $T$ is plotted which clearly gives very different Weiss temperature for the two directions, $\left(\theta_{W}\right)_{a b}=-176(20) \mathrm{K}$ and $\left(\theta_{W}\right)_{c}=-40(10) \mathrm{K}$ and $C=0.45$ and 0.47 respectively. This clearly indicates a anisotropic Weiss temperature in $\mathrm{Na}_{2} \mathrm{IrO}_{3}$. This fitting was tried with fixed $C$ which also resulted in same highly anisotropic $\theta_{W}$. This is very much expected for a quasi 2D system where magnetic exchange perpendicular to easy-plane (the plane where all the spins are lying upon) is much smaller than the same along the easy-plane (here $a b$-plane). Hence, origin of the small magnetic anisotropy in $\mathrm{Na}_{2} \mathrm{IrO}_{3}$ is both $g$-factor anisotropy and anisotropic exchange interaction.

\subsubsection{2 $\quad \mathrm{Li}_{2} \mathrm{IrO}_{3}$}

Earlier all magnetic measurements reported that $\mathrm{Li}_{2} \mathrm{IrO}_{3}$ is paramagnetic down to $5 \mathrm{~K}$. [53, 56], Kobayashi et. al. showed some low temperature drop around $15 \mathrm{~K}$ in susceptibility measurement but a hysteresis was observed by them, long range AF ordering in their report were not very evident [54]. First time, a true long range AF ordering in $\mathrm{Li}_{2} \mathrm{IrO}_{3}$ without any hysteresis was reported by our group on the sample prepared with $\mathrm{LiCl}$ flux. [60] Magnetization of different batches of $\mathrm{Li}_{2} \mathrm{IrO}_{3}$ were measured to obtain best ordered sample. Here 


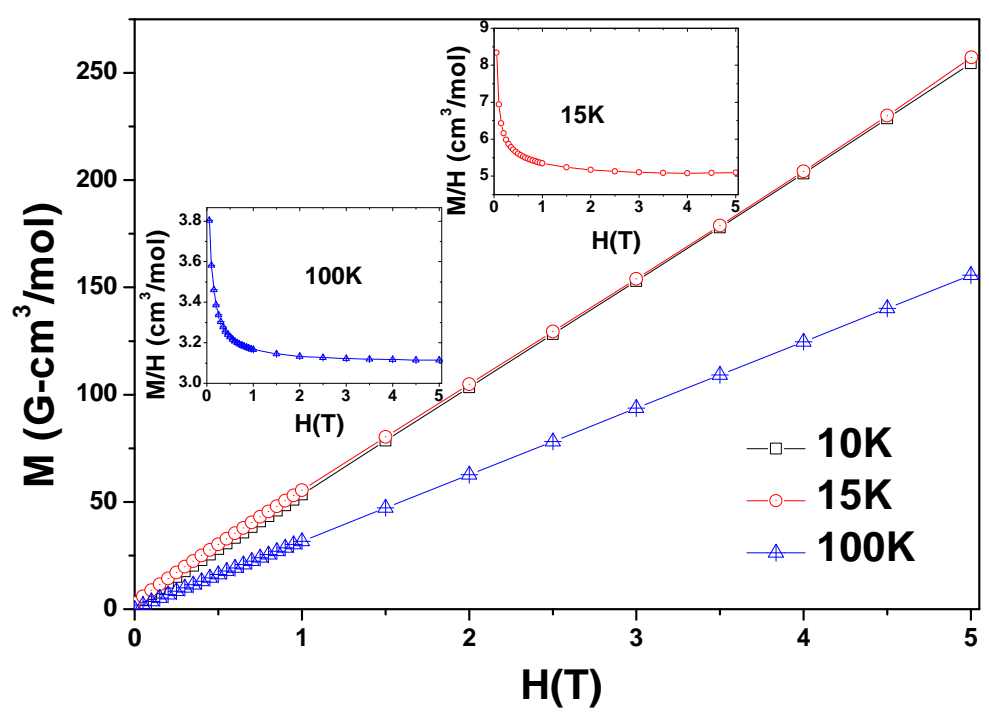

Figure 4.12: Isothermal magnetization $M$ versus magnetic field $H$ at various $T$. Inset a and b shows $M / H$ versus $H$ data measured at $T=15 \mathrm{~K}$ and $100 \mathrm{~K}$.

detailed analysis is shown for one of the best ordered sample. Fig. 4.11 shows magnetic susceptibility $\chi(T)$ versus $T$ data for $\mathrm{Li}_{2} \mathrm{IrO}_{3}$ measured at $1 \mathrm{~T}$. A drop in $\chi(T)$ is observed at low $T$, a zoomed plot of that $T$-region is shown in inset b. A $1 \%$ drop in the susceptibility is observed having maximum slop of the drop at $T=15 \mathrm{~K}$. This is characteristic feature for a long range $\mathrm{AF}$ ordering below $T_{N}=15 \mathrm{~K}$. Contrary to the $\mathrm{Na}_{2} \mathrm{IrO}_{3}$ behavior, in $\mathrm{Li}_{2} \mathrm{IrO}_{3} \chi(T)$ no broad hump is observed at low $T$. It indicates that quasi $2 \mathrm{D}$ behavior is lost in $\mathrm{Li}_{2} \mathrm{IrO}_{3}$ magnetism, may be due to more three dimensionality in the structure causing from shrinking of the lattice in the c-direction (c/a ratio has decreased in $\mathrm{Li}_{2} \mathrm{IrO}_{3}$ by $5 \%$ ). High temperature $\chi(T)$ versus $T$ data is fitted with CW law which give a small $\chi_{0}=$ $-7.3(7) \times 10^{-6} \mathrm{~cm}^{3} / \mathrm{mol}$ subtracting this value from all $\chi(T)$ difference susceptibility $\Delta \chi(T)$ is obtained. $1 / \Delta \chi$ versus $T$ is plottd in the inset a of the Fig. 4.11, CW fit of the data in the $T$-region $150-300 \mathrm{~K}$ gives $\mathrm{C}=0.39(1) \mathrm{cm}^{3} \mathrm{~K} / \mathrm{mol}$ corresponds to $\mu_{\text {eff }}=1.76 \mu_{B}$, very close to $\mathrm{S}_{\text {eff }}=1 / 2$ moment and $\theta_{W}=-44(8) \mathrm{K}$ represents AF exchange between Ir spins. Strength of the AF exchange has significantly gone down from $\mathrm{Na}_{2} \mathrm{IrO}_{3}$ to $\mathrm{Li}_{2} \mathrm{IrO}_{3}$ which also indicates that there is some FM components developing between Ir spins in Li-system compared to Na-system.

Isothermal magnetization $M(H)$ was measured at different $T$ on $\mathrm{Li}_{2} \mathrm{IrO}_{3}$ to check presence of any FM impurity. Fig. 4.12 shows that $M(H)$ is very linear upto $5 \mathrm{~T}$ at the temperature away from AF ordering, $\mathrm{T}=100 \mathrm{~K}$ and at the onset of the $\mathrm{AF}$ ordering, $\mathrm{T}=10$ and $15 \mathrm{~K}$. Inset $\mathrm{a}$ and $\mathrm{b}$ shows above $1 \mathrm{~T} M / H$ is constant independent of $H$. This confirms absence of any FM impurity. A slight curvature is observed in $M(H)$ data for $\mathrm{T}=10$ and 


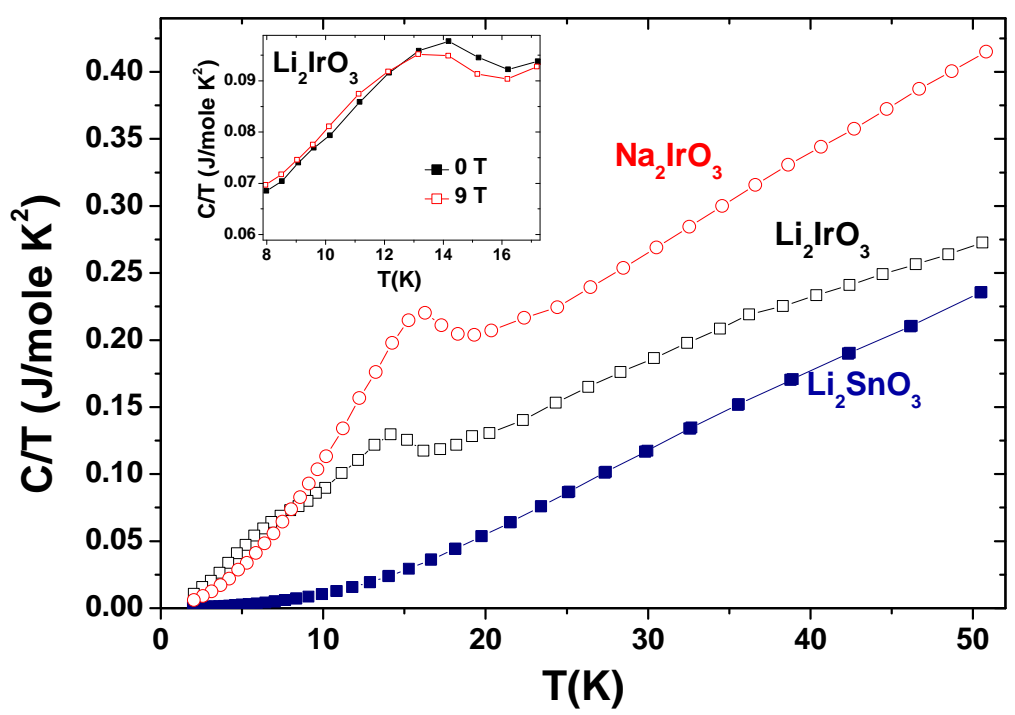

Figure 4.13: Heat capacity divided by temperature $C / T$ versus $T$ data between 2 to 50 $\mathrm{K}$ for $\mathrm{Na}_{2} \mathrm{IrO}_{3}$ and $\mathrm{Li}_{2} \mathrm{IrO}_{3}$ and of non-magnetic analog $\mathrm{Li}_{2} \mathrm{SnO}_{3}$. The inset shows $C / T$ versus $T$ measured at $\mathrm{H}=0$ and $9 \mathrm{~T}$ for $\mathrm{Li}_{2} \mathrm{IrO}_{3}$.

$15 \mathrm{~K}$ which may be due to the influence of magnetic ordering. It is also seen the Fig. 4.12 $M(H)$ data of $T=10 \mathrm{~K}$ is slightly lower than of $T=15 \mathrm{~K}$, this is due to long range AF ordering below $15 \mathrm{~K}$.

\subsubsection{Heat capacity}

Previously heat capacity measurement on $\mathrm{Na}_{2} \mathrm{IrO}_{3}$ single crystals had confirmed long range AF ordering below $T_{N}=15 \mathrm{~K}$ from a lambda like peak in $C / T$. [52] $\mathrm{Li}_{2} \mathrm{IrO}_{3}$ Heat capacity was measured on the polycrystalline powder after washing the $\mathrm{LiCl}$ flux, pelletizing the powder and re-sintered at $700^{\circ}$ C. Fig. 4.13 shows comparative study of the heat capacity divided by temperature $C / T$ versus $T$ for the $\mathrm{Na}$ and $\mathrm{Li}$-system and same data for non-magnetic analog of Li-system, $\mathrm{Li}_{2} \mathrm{SnO}_{3}$. Both $\mathrm{Na}_{2} \mathrm{IrO}_{3}$ and $\mathrm{Li}_{2} \mathrm{IrO}_{3}$ shows an anomaly around $15 \mathrm{~K}$ in $C / T$ which confirms long range AF ordering around. A small cusp is also observed around $C / T$ at $5 \mathrm{~K}$ for $\mathrm{Li}_{2} \mathrm{IrO}_{3}$. This is most likely arise due to a small $(\leq 5 \%)$ disorder present in the sample which is discussed in more detail in the section Disorder in $A_{2} I_{r} O_{3}$. Inset shows magnetic field dependence of $C / T$ for $\mathrm{Li}_{2} \mathrm{IrO}_{3}$, by the effect of $9 \mathrm{~T}$ magnetic anomaly shifts down by $2 \mathrm{~K}$ which confirms bulk magnetic ordering is antiferromagnetic type, same was also observed for $\mathrm{Na}_{2} \mathrm{IrO}_{3}$ and reported by Singh et. al. before [52].

The magnetic heat capacity $(\Delta C)$ was obtained by subtracting lattice heat capacity (non-magnetic analog, $\mathrm{Li}_{2} \mathrm{SnO}_{3}$ for Li-system) from the sample heat capacity $(C)$. Fig. 4.14 


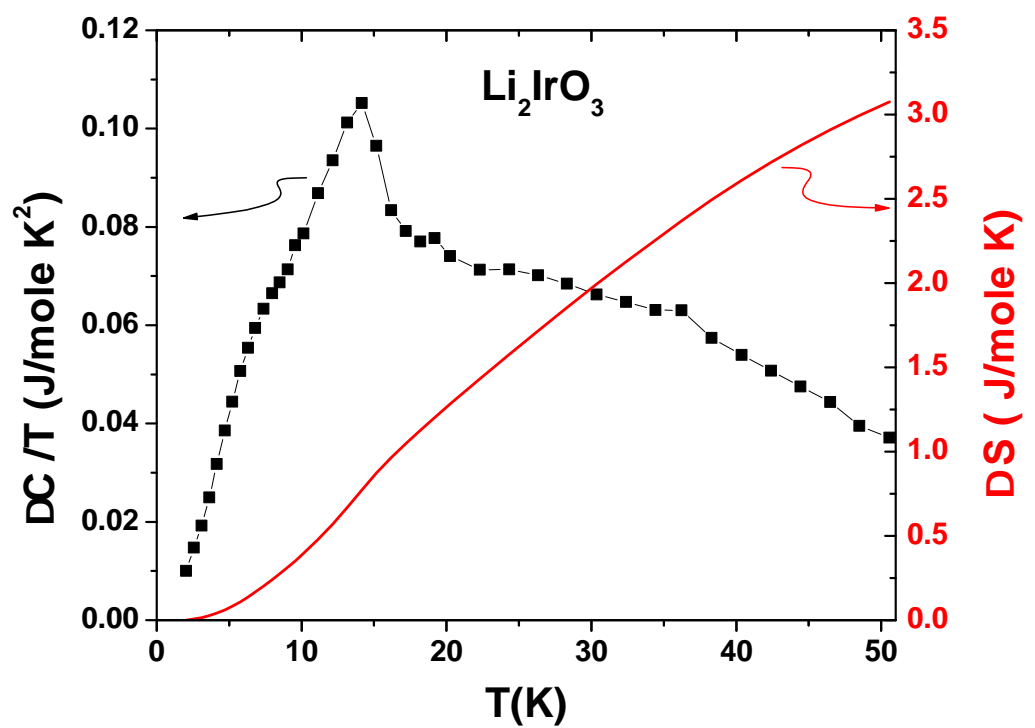

Figure 4.14: Left axis data shows magnetic heat capacity divided by temperature $\Delta C / T$ versus $T$ for $\mathrm{Li}_{2} \mathrm{IrO}_{3}$. Right axis shows magnetic entropy $\Delta S$ versus $T$ for the same.

shows variation of magnetic heat capacity divided by temperature, $\Delta C / T$ versus $T$ for $\mathrm{Li}_{2} \mathrm{IrO}_{3}$ where a lambda like peak at $15 \mathrm{~K}$ is clearly visible and $\Delta C / T$ drops towards zero below that. Magnetic entropy $\Delta S$ is calculated by integrating this curve over the plotted temperature range. At $\mathrm{T}_{N}$ value of $\Delta S$ is $0.85 \mathrm{~J} /$ mole $\mathrm{K}$ which is only about $15 \% \mathrm{R} \ln 2$. A reduced entropy was also observed in case of single crystalline $\mathrm{Na}_{2} \mathrm{IrO}_{3} .[52]$ It indicates also a reduced ordered moment. Even upto $50 \mathrm{~K}$ the whole $S=1 / 2$ entropy is not recovered. Reduced ordered moment and spreading of magnetic entropy much above $T_{N}$ clearly tells that the system is highly frustrated system and here frustration is not only geometric but a dynamical frustration of strong Kitaev interactions. Weiss temperature can reflect only average of different interactions not the exact strength of the individual exchange interaction and due to this reason total magnetic entropy is not recovered upto the temperature equivalent to $\left|\theta_{W}\right|=45 \mathrm{~K}$ (representing strength of the AF exchange interaction). Sometimes such a strong frustration pushes magnetic systems near to a non-magnetic ground state, observed reduced moment in $\mathrm{Li}_{2} \mathrm{IrO}_{3}$ can also be cause of that.

\subsection{Disorder effect in $\mathrm{A}_{2} \mathrm{IrO}_{3}$}

Different disorder effect in $\mathrm{A}_{2} \mathrm{IrO}_{3}$ crystal structure is a huge barrier to realize real magnetic ground state. $\mathrm{Na}_{2} \mathrm{IrO}_{3}$ polycrystal, first obtained in our group showed spin-glass transition below $6 \mathrm{~K}$, only much later, long range AF ordered was observed in the $\mathrm{Na}_{2} \mathrm{IrO}_{3}$ polycrystal. 


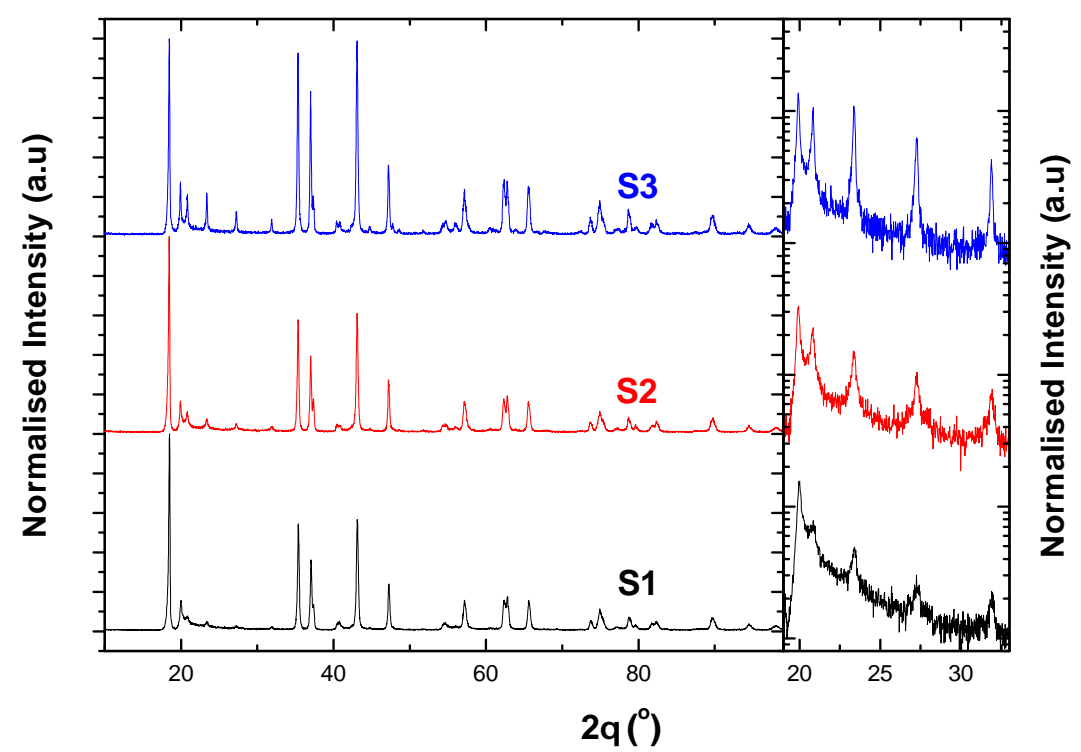

Figure 4.15: (Left) PXRD of three type of $\mathrm{Li}_{2} \mathrm{IrO}_{3}$ sample: disordered( $\left.S 1\right)$, partially ordered $(S 2)$, highly ordered $(S 3)$ for entire $2 \theta$-region. (Right) Zoomed view of them for the $2 \theta$-region 19 to $33^{\circ}$ in $\log$ scale.

Previous reports on $\mathrm{Li}_{2} \mathrm{IrO}_{3}$ also reported non-magnetic ground state. Kobayashi et.al [54] had reported magnetic disorder in $\mathrm{Li}_{2} \mathrm{IrO}_{3}$ due to a real site disorder between $4 \mathrm{~h}$ and $2 \mathrm{a}$ site. But our NMR study found $\left[\mathrm{A}_{1 / 3} \mathrm{Ir}_{2 / 3}\right]$ layers are intact no site disordered is observed in our well magnetically ordered system.[69] Also previous NMR work on $\mathrm{Li}_{2} \mathrm{MnO}_{3}$ have concluded the same.[70].

Later O'Malley et.al also reported that principle origin of disorder in all $\mathrm{Li}_{2} \mathrm{MO}_{3}(\mathrm{M}=$ TM) is due to a fault in the stacking of honeycomb layer. $[55,56]$ They considered three type of faults, one translational and other two rotational fault. They simulated PXRD patterns with different degree of stacking fault using DIFFaX and showed that effect of stacking fault is a continuous slope in PXRD pattern in the $2 \theta$ region 19 to $33^{\circ}$ namely, Warren fall and a pick broadening in the same region. But to account this stacking fault in Rietveld analysis, a partial site exchange between between $4 \mathrm{~h}$ and $2 \mathrm{a}$ is used which only reduces the height of the PXRD peaks in this $2 \theta$ region but can not catch the real effect of continuous slope and peak broadening as observed by O'Malley et.al and also by us (Fig. 4.15).

We have studied effect of this disorder on the magnetic and thermodynamic properties also. To compare these, three different polycrystalline samples are discussed: $\operatorname{disordered}(S 1)$, partially ordered $(S 2)$, highly ordered $(S 3)$. S1 and S2 were prepared in solid sate reaction method, sintered at 900 and $1000^{\circ} \mathrm{C}$ respectively, S3 was prepared in $\mathrm{LiCl}$ flux. In the disordered S1 sample, Warren fall in the $19^{\circ}$ to $33^{\circ} 2 \theta$-region is extremely large, almost no 


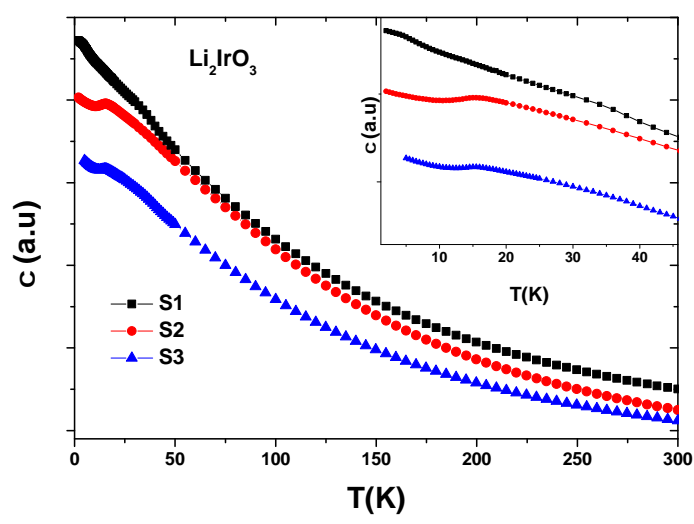

Figure 4.16: Magnetic susceptibility $(\chi)$ versus $T$ of disordered $(S 1)$, partially ordered $(S 2)$, highly ordered $(S 3)$ $\mathrm{Li}_{2} \mathrm{IrO}_{3}$ samples. Inset shows closer look at low- $T$ showing magnetic ordering in $S 2$ and $S 3$.

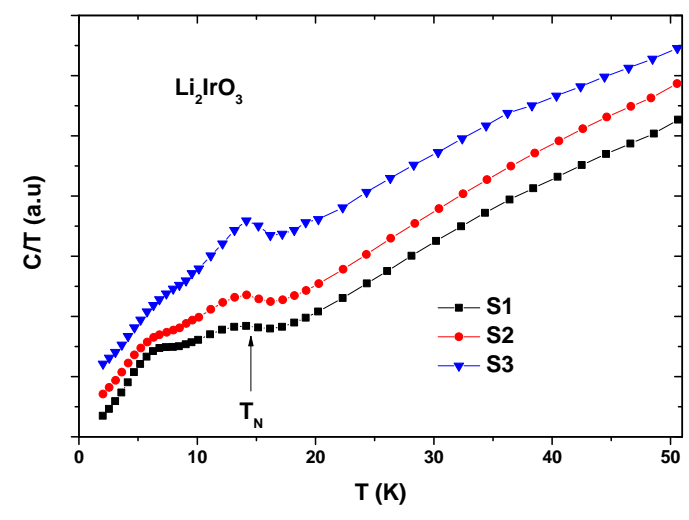

Figure 4.17: Heat capacity divided by temperature $C / T$ versus $T$ of disordered $(S 1)$, partially ordered $(S 2)$, highly ordered $(S 3) \quad \mathrm{Li}_{2} \mathrm{IrO}_{3}$ samples. Vertical arrow marks $T_{N}$.

peaks are visible other than at $20^{\circ}$ (Fig. 4.15). This tendency reduces in the S2 sample (Fig. 4.15), it has still some amount of Warren fall and peak broadening in that $2 \theta$-region but intensity of other 4 peaks have also increased. Different peaks at $40^{\circ}$ and near $55^{\circ}$ are also separate and visible in S2 sample. In the S3 sample, intensities of the PXRD peaks in the $2 \theta$-region $19^{\circ}$ to $33^{\circ}$ have significantly increased as well as peaks are very sharp now (Fig. 4.15). This suggest that S3 is a very well 3D ordered sample where coherent ordering of the honeycomb layer is obtained.

As expected there is no magnetic ordering in the disordered S1 sample, no drop in susceptibility near $15 \mathrm{~K}$. But for both S2 and S3 sample there is a drop in susceptibility around $15 \mathrm{~K}$, signifying the presence of long range AF ordering below $15 \mathrm{~K}$ (Fig. 4.16). High temperature susceptibility is very similar for all these three batches, they give effective $S=1 / 2$ moment $\mu_{e f f} \approx 1.78(11) \mu_{B}$ and $\theta_{W} \approx-40(10) \mathrm{K}$ by $\mathrm{CW}$ fitting. This again confirms that individual honeycombs are unaffected and there is no site disorder. Significant difference is found in heat capacity measurements of S1, S2 and S3 samples (Fig. 4.17). S1, which showed no ordering in magnetic measurement has a broad hump around $5 \mathrm{~K}$ in $C / T$ and a very weak feature at $15 \mathrm{~K}$. In S2, broad feature around $5 \mathrm{~K}$ becomes weaker and a sharper peak like feature appear at $15 \mathrm{~K}$ which signifies long range ordering, though a part of magnetic entropy still present near $5 \mathrm{~K}$. In the well ordered sample S3, a sharp peak is visible in $C / T$ at $15 \mathrm{~K}$, hump near $5 \mathrm{~K}$ is almost invisible. Hence almost all the entropy from $5 \mathrm{~K}$ shifts to $T_{N}$ in $\mathrm{S} 3$.

This comparative analysis on $\mathrm{Li}_{2} \mathrm{IrO}_{3}$ shows that lower temperature sintered samples has 


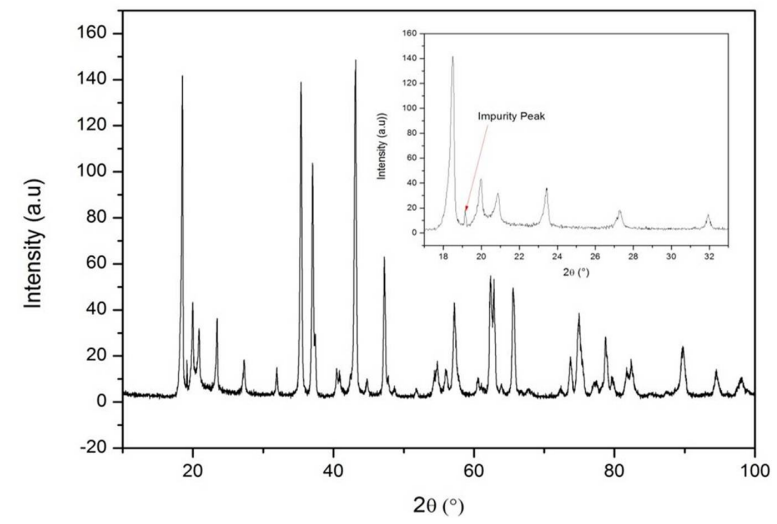

Figure 4.18: $\mathrm{PXRD}$ of $\mathrm{Li}_{2} \mathrm{IrO}_{3}$ sintered at higher temperature than $1000^{\circ}$, (inset) closer look at the secondary phase peak.

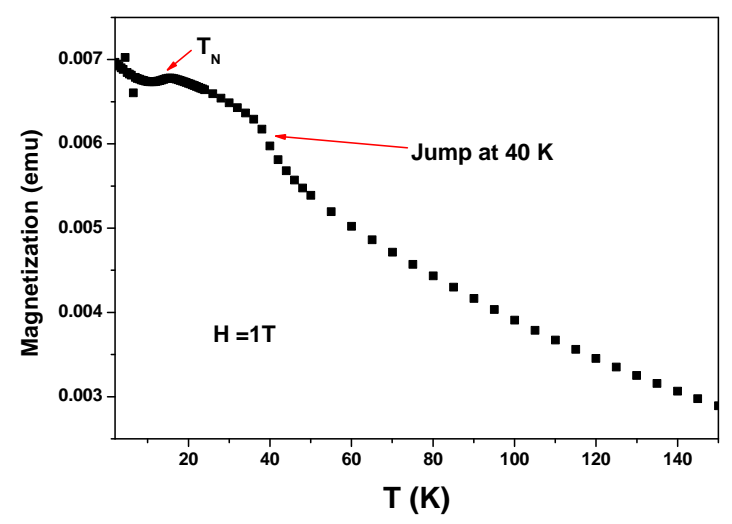

Figure 4.19: Magnetization versus $T$ of two phase $\mathrm{Li}_{2} \mathrm{IrO}_{3}$ sample. Having a kink around $40 \mathrm{~K}$ with drop at $\mathrm{T}_{N}$.

higher degree of stacking fault and it reduces mostly with increasing sintering temperature but best structural ordering is achieved only when one reaches higher temperature in Li environment to avoid escape of $\mathrm{Li}$ from the phase. Lowered ordered sample shows a short range magnetic ordering and 3D long range AF ordering is only achieved when stacking fault in the stacking of the honeycomb layers reduces to almost zero and a coherent ordering of the layers is set up. But high temperature may not not be the best solution to achieve best ordered $\mathrm{Li}_{2} \mathrm{IrO}_{3}$.

We tried to go even higher temperature in Li environment to totally abolish any stacking disorder. But appearance of some secondary phase in the XRD has been detected(Fig. 4.18). Inset of the figure clearly points to a secondary peak in PXRD at around $2 \theta=19^{\circ}$ just above (001) peak which does not belong to $\mathrm{C} 2 / \mathrm{m}$ crystal structure. In magnetization also a shoulder around $40 \mathrm{~K}$ (Fig. 4.19) is observed above $\mathrm{T}_{N}$, but the drop in magnetization at $T=15 K$ due to AF ordering is still present. Susceptibility increases by few times in magnitude. At high temperature unknown phase is formed and which could be some other poly-morph of $\mathrm{Li}_{2} \mathrm{IrO}_{3}$ or totally some other Li-Ir-O phase. This high- $T$ phase was also synthesized in the phase pure form. It is not a honeycomb lattice of Ir rather a disconnected honeycomb network in $3 \mathrm{D}$. This was named $\beta$ - $\mathrm{Li}_{2} \mathrm{IrO}_{3}$ and structure is hyper-honeycomb like hyperkagome.[71] Preliminary results on the phase are shown in Appendix A. This finding clearly suggests that there exist a high temperature poly-morph of $\mathrm{Li}_{2} \mathrm{IrO}_{3}$ just 50$100^{\circ}$ above the honeycomb- $\mathrm{Li}_{2} \mathrm{IrO}_{3}$ phase. For single-crystal synthesis like $\mathrm{Na}_{2} \mathrm{IrO}_{3}$ in grain growth method, the system has to be kept at higher temperature for a long time but for $\mathrm{Li}_{2} \mathrm{IrO}_{3}$ it results in a different poly-morph, a structural change. Different flux were also tried e.g. combination of different lithium borate flux, carbonate flux, halide fluxes; only 


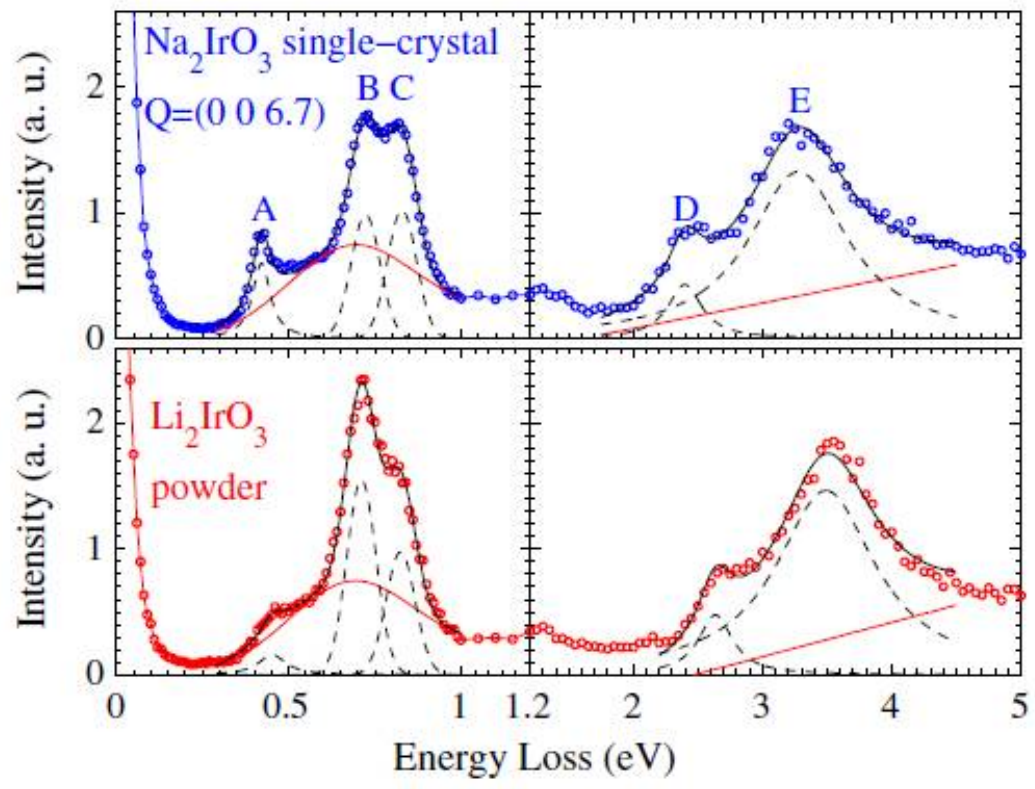

Figure 4.20: (Top) Wide energy rane RIXS spectrum for a single-crystal sampleof $\mathrm{Na}_{2} \mathrm{IrO}_{3}$ at $\mathbf{Q}=\left(\begin{array}{lll}0 & 0 & 6.7\end{array}\right)$ with $\mathrm{E}_{i}=11.217 \mathrm{keV}$. (Bottom) RIXS spectrum for $\mathrm{Li}_{2} \mathrm{IrO}_{3}$ powder sample at $|\mathbf{Q}| \approx 8 \AA$, and with same $\mathrm{E}_{i}$. All the spectrum obtained at room temperature. Measurement was done by Gretarsson et. al. on our synthesized sample, spectrum is reprinted from [58]

$\mathrm{LiCl}$ flux partially worked to give very well ordered polycrystal of $\mathrm{Li}_{2} \mathrm{IrO}_{3}$ but no single crystal of this phase till date is obtained.

\section{5 $\quad \mathrm{A}_{2} \mathrm{IrO}_{3}$ electronic structure}

To determine the electronic structure of honeycomb lattice iridate H. Gretarsson et. al. performed resonant inelastic x-ray spectroscopy (RIXS) experiment on $\mathrm{Li}_{2} \mathrm{IrO}_{3}$ well ordered polycrystal prepared by myself and $\mathrm{Na}_{2} \mathrm{IrO}_{3}$ single crystal grown by Y. Singh. The RIXS process at the $L_{3}$ edge of $\operatorname{Ir}$ (or any other d electron system) is a second order process consisting of two dipole transitions $(2 \mathrm{p} \rightarrow 5 \mathrm{~d}$ followed by $5 \mathrm{~d} \rightarrow 2 \mathrm{p})$. Therefore, it is especially valuable for detecting excitations between the $d$ levels and has been extensively utilized in the study of $3 \mathrm{~d}$ transition metal compounds. [58]. Main electronic feature near Fermi energy for a d-electron system is dominated by d-electron DOS so to understand electronic structure of $\mathrm{A}_{2} \mathrm{IrO}_{3}$, splitting of $\mathrm{Ir} 5 \mathrm{~d}$ orbitals needs to be determined.

Fig. 4.20 represents high-resolution RIXS spectrum for $\mathrm{Na}_{2} \mathrm{IrO}_{3}$ (top) and $\mathrm{Li}_{2} \mathrm{IrO}_{3}$ (bottom) plotted in a wide energy scale, obtained by Gretarsson et. al.[58] This scan was obtained at room temperature and plotted as a function of energy loss $\left(\omega=\mathrm{E}_{i}-\mathrm{E}_{f}\right)$. The incident 

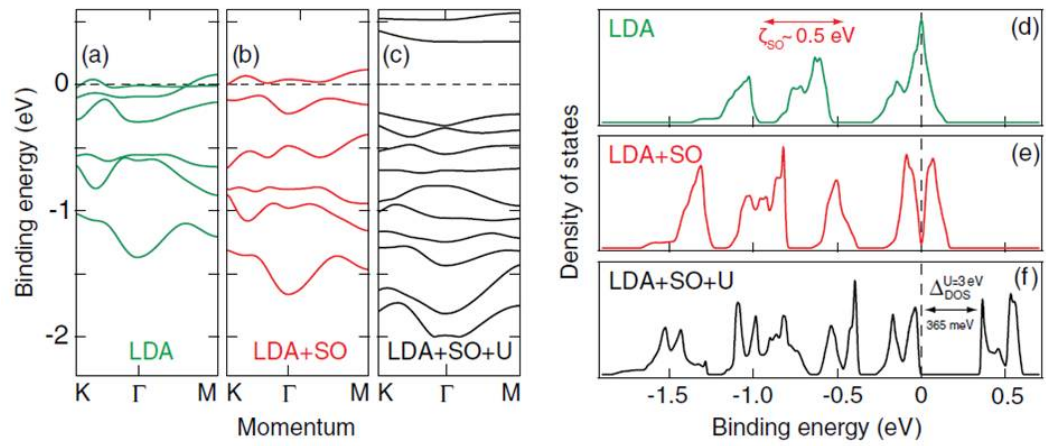

Figure 4.21: (a)(c) Ir $5 \mathrm{~d} t_{2 g}$ band structure $\left(k_{z}=0\right)$, and (d)(f) corresponding DOS, obtained with $\mathrm{LDA}, \mathrm{LDA}+\mathrm{SO}$, and $\mathrm{LDA}+\mathrm{SO}+\mathrm{U}\left(U=3 \mathrm{eV}, J_{H}=0.6 \mathrm{eV}\right)$ for $\mathrm{Na}_{2} \mathrm{IrO}_{3}$, reprinted from [61]

energy, $\mathrm{E}_{i}=11.217 \mathrm{keV}$, was chosen to maximize the resonant enhancement of the spectral features of interest below $1 \mathrm{eV}$. Both the spectra were fitted with 5 peaks (labeled A-E). To understand the nature of the excitations which has resulted these peaks, multiconfiguration self-consistent-field and multireference configuration-interaction (MRCI) calculations were carried out on clusters consisting of one central $\mathrm{IrO}_{6}$ octahedron, all adjacent $\mathrm{Na}$ or $\mathrm{Li}$ ions, and the three nearest-neighbor $\mathrm{IrO}_{6}$ octahedra. Local d-d transitions were calculated for central $\mathrm{IrO}_{6}$ octahedron.[58] Comparing with MRCI and SOC calculation, the peak B and $\mathrm{C}$ (Fig. 4.20) are identified as due to transition within $\mathrm{t}_{2 g}$ between $J_{\text {eff }}=3 / 2$ to $1 / 2$ state. Broad peaks D and $\mathrm{E}$ in between 2.5-3.5 eV (Fig. 4.20) are resulting from multipole $\mathrm{t}_{2 g}$ to $\mathrm{e}_{g}$ transition.[58] The splitting of the strong RIXS peak located at $0.70 .8 \mathrm{eV}$ (B and C) is due to the trigonal distortion which is well corroborated with MRCI+SOC calculations. This splitting of $110 \mathrm{meV}$ is much smaller than the SOC of $0.40 .5 \mathrm{eV}$ strongly suggests that there is no mixing between $J_{\text {eff }}=3 / 2$ and $J_{\text {eff }}=1 / 2$. Hence these two principal d-d transitions (B,C and D,E) observed within $5 d$ orbitals confirm spin-orbit excitation scenario, proposed by Kim et. al, depicted in Fig. 2.3.[10] So $\mathrm{A}_{2} \mathrm{IrO}_{3}$ can be called a spin-orbit relativistic insulator. Now whether it is a band insulator or a Mott insulator or a Slatar insulator was verified by angle resolved photo emission spectroscopy (ARPES) and band structure calculation by our collaborator Ricardo Comin et. al.

ARPES measurement was done on the single crystal grown by Yogesh Singh in our lab.[61] ARPES observed zero spectral weight at Fermi energy $\left(\mathrm{E}_{F}\right)$ and spectra near $\mathrm{E}_{F}$ were fitted with four Gaussian peak and they are Ir $5 \mathrm{~d}^{-\mathrm{t}_{2 g}}$ spectra. This opening of the gap happen much above $T_{N}=15 \mathrm{~K}$ and it does not show any temperature dependence around $\mathrm{T}_{N}$ which directly excludes any magnetic-order-driven nature of the insulating sate like Slater insulator. Insulating gap for $\mathrm{Na}_{2} \mathrm{IrO}_{3}$ was measured from the potassium induced chemical potential shift and optical conductivity measurement at room temperature and both the 
experiment determine charge gap $\Delta_{\text {gap }}=340 \mathrm{meV}$. Now whether this is a band insulator or a Mott insualtor that only can be understood by relative importance of Coulomb Repulsion $U$ and spin-orbit coupling $(S O)$ to open the gap which can be analytically encountered by density functional theory (DFT) calculation by our theoretical collaborators. DFT was done by both GGA [58] and LDA [61] approach and both of them gave similar conclusion. Here in Fig. 4.21 (a) - (c), Ir $\mathrm{t}_{2 g}$ band structure $\left(\mathrm{k}_{z}=0\right)$ and in (d) - (f), corresponding DOS for $\mathrm{Na}_{2} \mathrm{IrO}_{3}$ are presented for $\mathrm{LDA}, \mathrm{LDA}+\mathrm{SO}, \mathrm{LDA}+\mathrm{SO}+\mathrm{U}$ approach which was done by Comin et. al.[61] Without any spin-orbit coupling $S O$ and $U$ a huge DOS is present at $\mathrm{E}_{F}$ and it is metallic due to partially filled $t_{2 g}$ [Fig. 4.21 (a)and (d)] which is contradictory to experimental result. Now if a $\mathrm{SO}$ is switched on, in LDA+SO a clear gap opens up at $\mathrm{E}_{F}$ in the $\mathrm{k}_{z}=0$ band dispersion [Fig. 4.21(b)], although in the DOS only a zero gap can be observed [Fig. 4.21(e)]. Disagreement between observed and calculated gap even in $\mathrm{LDA}+\mathrm{SO}$ reveals that $\mathrm{Na}_{2} \mathrm{IrO}_{3}$ can not be treated as relativistic band insulator. True size of the gap only can be achieved in DFT if a $U=3 \mathrm{eV}$ is applied in $\mathrm{LDA}+\mathrm{SO}+\mathrm{U}$ [Fig. 4.21 (c)and

(f)]. This confirms a important role of $U$ in opening the gap in $\mathrm{Na}_{2} \mathrm{IrO}_{3}$, validating Mott scenario. For a $5 \mathrm{~d}$ orbital $U=3 \mathrm{eV}$ might seem a large value, however effective reduction of $U$ depends upon the polarizability of the surrounding medium.[61] Exactly same type of gap opening is observed for $\mathrm{Li}_{2} \mathrm{IrO}_{3}$ in $G G A+S O+U$ approach.[58]

Hence detail electronic structure study proves $J_{\text {eff }}=1 / 2$ spin-orbit Mott insulating state in both $\mathrm{Na}_{2} \mathrm{IrO}_{3}$ and $\mathrm{Li}_{2} \mathrm{IrO}_{3}$; this Mott insulating state is driven by the delicate interplay between $U$ and $\mathrm{SO}$ energy scales in which structural distortion also playing crucial participating role.

\section{6 $\quad \mathrm{A}_{2} \mathrm{IrO}_{3}$ magnetic structure}

\subsection{1 $\quad \mathrm{Na}_{2} \mathrm{IrO}_{3}$}

$\mathrm{Na}_{2} \mathrm{IrO}_{3}$ magnetic structure was first predicted from resonant x-ray magnetic scattering (RXMS) measurement by Liu et. al. performed at It $L_{3}$ edge.[72] Well-defined peaks were observed at $(0 \pm 1 \mathrm{~L})$ Bragg points with $(\mathrm{L}=7,9,11)$ which are structurally forbidden peak for $\mathrm{Na}_{2} \mathrm{IrO}_{3}$. From the temperature dependence of the resonant peak ( $\left.\begin{array}{lll}0 & 1 & 11\end{array}\right)$ it was concluded to be originated from magnetic ordering.[72] From the azimuthal dependence of this peak it was concluded that moments are predominantly along crystalographic $a$ direction. Also size of the magnetic unit cell was suggest to be same as structural unit cell. Two possibility were discussed stripy and zig-zag spin structure. But from the spin density 
a) Néel

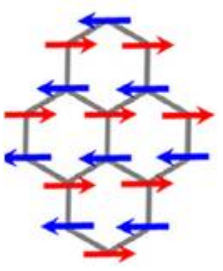

b) zigzag

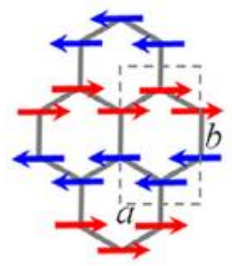

c) stripy

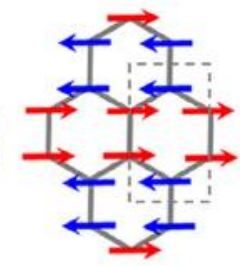

d) $\bigcirc \quad$ Néel

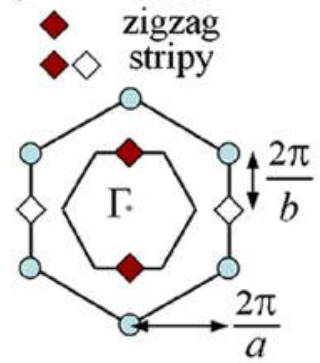

Figure 4.22: (a) Néel (b) zig-zag (c) stripy spin structure in honeycomb lattice. (d) Position of the magnetic Bragg peaks in reciprocal space for different spin-configurations (inner hexagon represents first Brillouin zone of the honeycomb lattice). Reprinted from [36].

functional theory calculation it was concluded that zig-zag spin-structure has lower energy so it is the true magnetic structure of $\mathrm{Na}_{2} \mathrm{IrO}_{3} \cdot[72]$

Concurrently, dispersion of the spin-wave excitation was studied by inelastic neutron scattering (INS) by Choi et. al. on $10 \mathrm{~g} \mathrm{Na}_{2} \mathrm{IrO}_{3}$ powder sample prepared in our lab.[36] Inelastic signal showed a sinusoidal like dispersive boundary extrapolating to $Q_{(010)}=0.67$ $\AA^{-1}$ at the lowest energy. It is much lower than the expected for the Néel structure $Q_{(010)}=$ $1.34 \AA^{-1}$. This $Q$-value matches both with the stripy and zig-zag spin structure. Fig. 4.22 shows the different spin structures: (a) Néel (b) zig-zag (c) stripy. Difference between zigzag and stripy structure is very small: zig-zag can be depicted as antiferromagnetically coupled ferromagnetic spin chains and stripy is ferromagnetically coupled antiferromagnetic spin chains, if viewed along $a$-crystallographic axis of honeycomb lattice. Another small difference is that coupling between shortest Ir-Ir distance in $\mathrm{Na}_{2} \mathrm{IrO}_{3}$ is ferromagnetic in case of zig-zag and it is antiferromagnetic in case of stripy. Stripy also has a magnetic Bragg peak outside the first Brillouin zone (BZ) whereas zig-zag has only on the first BZ (Fig. 4.22 d). Thats why dispersion of spin-wave also varies significantly for both of them, according to $\mathrm{J}_{1}-\mathrm{J}_{2}-\mathrm{J}_{3}$ model and $\mathrm{KH}$ model stripy spin structure shows a quadratic dispersion boundary opposite to the observed sinusoidal boundary. But calculated dispersion boundary from zig-zag structure in $\mathrm{J}_{1}-\mathrm{J}_{2}-\mathrm{J}_{3}$ exactly fit with the observed one hence, zig-zag spin structure confirmed for $\mathrm{Na}_{2} \mathrm{IrO}_{3}$ through INS.[36]

Later magnetic propagation vector in reciprocal space was directly determined by elastic neutron scattering, $\vec{q}_{m}=(0,1,0.5)$.[57] But one can observe a high intensity at this $\vec{q}_{m}$ for both stripy and zig-zag. Though for stripy strongest intensity should be observed at $\vec{q}_{m}=$ $(0,3,0.5)$ which was reported zero by Ye et. al. for the measurement at lowest $T$. Hence 


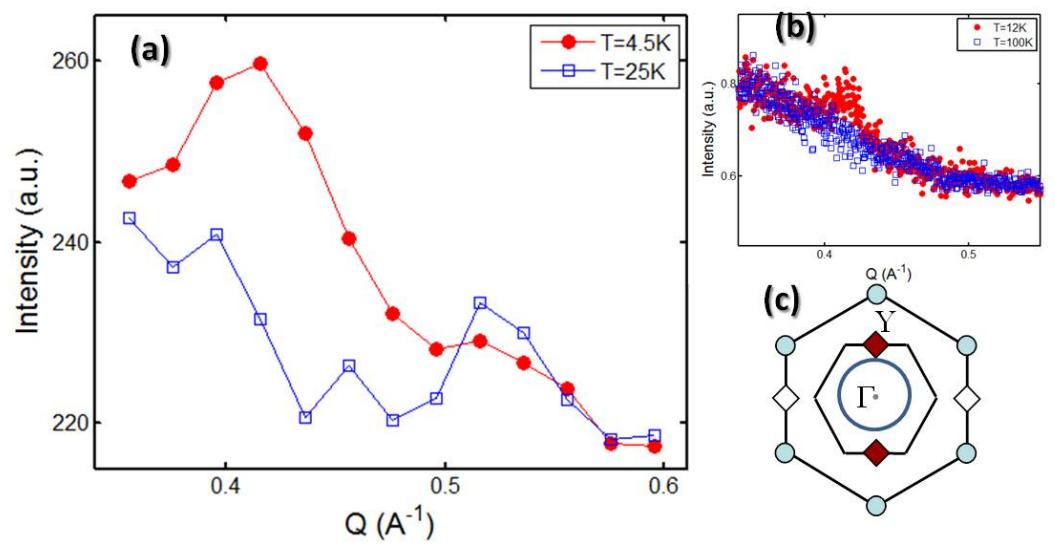

Figure 4.23: (a) Inegrated intensity of all elastic lines in inelstic neutron scattering (INS) versus Q-value $(A)$, measured at $T=4.5 \mathrm{~K}$ and $25 \mathrm{~K}$ on $\mathrm{Li}_{2} \mathrm{IrO}_{3}$. (b) Raw data of neutron diffraction measured at $T=12 \mathrm{~K}$ and $100 \mathrm{~K}$, intensity versus Q-value $(\AA)$. (c) Projection of the magnetic Bragg peak in reciprocal space of honeycomb lattice (here, the blue ring for $\mathrm{Li}_{2} \mathrm{IrO}_{3}$ ) The plots were obtained from private communication with Dr. R. Coldea [73].

elastic neutron scattering directly confirms that real magnetic structure of $\mathrm{Na}_{2} \mathrm{IrO}_{3}$ is zig-zag (Fig. 4.22.b).[57]

\subsection{2 $\quad \mathrm{Li}_{2} \mathrm{IrO}_{3}$}

Very recently inelastic neutron scattering has been done on $\mathrm{Li}_{2} \mathrm{IrO}_{3}$ by Choi et. al. on the $10 \mathrm{~g}$ well ordered polycrystalline sample prepared by myself in many batches. Then they were all characterized to prove best magnetic and structural ordering before they weer sent for INS experiment. The $10 \mathrm{~g}$ sample was spread inside the annular region of a hollow annular cylinder of annular thickness of $\leq 1 \mathrm{~mm}, 50 \mathrm{~mm}$ height and $40 \mathrm{~mm}$ outer diameter. Ir is a strong neutron absorber so to get maximum intensity such a geometry was used to maximize surface area. Then scattering data was recorded at different energy , temperature and $Q$-value region for several hours. Fig. 4.23. (b) shows the raw data of neutron diffraction measured at $T=12 \mathrm{~K}$ (below $T_{N}=15 \mathrm{~K}$ ) and $100 \mathrm{~K}$ (above $T_{N}$ ). A significant peak-intensity observed at $Q=0.4 \AA^{-1}$ for $T=12 \mathrm{~K}$ which goes to zero at higher temperature. As any structural change is not observed in heat capacity, this must be coming from magnetic Bragg-reflection. Inelastic neutron scattering was also done at $T=4.5 \mathrm{~K}$ and 25 K. In Fig. 4.23. (a) integrated intensity for all elastic lines is plotted which shows a peak at $Q=0.4 \AA^{-1}$ for measurement below $T_{N}$ and that peak is gone at $T=25 \mathrm{~K}$. So neutron diffraction and inelastic neutron scattering confirms a magnetic Bragg peak at $Q \approx 0.4 \AA^{-1}$. If this $Q$-value is projected in reciprocal space it is inside 1st $\mathrm{BZ}$ of the Honeycomb lattice, Bragg peak for $\mathrm{Li}_{2} \mathrm{IrO}_{3}$ is situated on the Blue circle shown inside $1^{\text {st }} \mathrm{BZ}$ (Fig. 4.23 (c)) . It 
means that in real space magnetic unit cell is much bigger, may be some incommensurate magnetic structure. Comparing with same $J_{1}-J_{2}-J_{3}$ model it may be some spiral variation of zig-zag magnetic structure.

\subsection{Discussion}

Synthesis and study of physical property of phase pure honeycomb lattice iridate $\mathrm{Li}_{2} \mathrm{IrO}_{3}$, sister compound of previously synthesized $\mathrm{Na}_{2} \mathrm{IrO}_{3}$ was one of the early achievement of this project. Origin of disorder in these class of materials and the effect on magnetism is also first time identified. Study of bulk magnetic, thermodynamic and electrical property and scattering experiments on $\mathrm{A}_{2} \mathrm{IrO}_{3}$ added with band structure calculation clearly identified them as novel $\mathrm{J}_{\text {eff }}=1 / 2$ spin-orbit Mott insulator. Local probes like $\mu$-SR and NMR also unambiguously identified local footprints of magnetic ordering in both of these systems. Work on both these honeycomb lattice iridates were started to realize proposed Kitaev spin liquid state but in reality it is proved to be magnetically ordered system having unconventional magnetic structures. The HK model was unable to explain the magnetic structure of both $\mathrm{Na}_{2} \mathrm{IrO}_{3}$ and $\mathrm{Li}_{2} \mathrm{IrO}_{3}$, there were many extensions but framing both of them in a single physical model is still not achieved. First one needs to understand the cause of deviation from HK model. One structural origin of deviation is definitely found that Ir-O-Ir bond angle is not $90^{\circ}$ in both $\mathrm{Na}$ and Li-system which is desired for ideal Kitaev interaction. In Li-system it is nearer to $90^{\circ}$ than for Na-system but not exactly $90^{\circ}$. Cause of this deviation is trigonal distortion of $\mathrm{IrO}_{6}$ octahedra which resulted some extra splitting of $\mathrm{t}_{2 g}$ orbital but not enough to destroy its $J_{\text {eff }}=1 / 2$ scenario. Apart from this, HK model also does not treat interaction beyond nearest neighbor between Ir-spins. It is highly probable for such a closed honeycomb structure of Ir-spins in $\mathrm{A}_{2} \mathrm{IrO}_{3}$ to have beyond nearest neighbor exchange interactions. This chapter has described all the magnetic and electronic property of honeycomb lattice iridate but to understand the microscopic origin of its magnetic interactions we have to perturb the system structurally and magnetically and see the effect of it on $\mathrm{A}_{2} \mathrm{IrO}_{3}$. This perturbation can be done by different doping at magnetic and non-magnetic site. The doping effect is described in the next chapter. 



\section{Chapter 5}

\section{Doping effect on $\mathrm{A}_{2} \mathrm{IrO}_{3}$}

Both honeycomb lattice iridates $\mathrm{Na}_{2} \mathrm{IrO}_{3}$ and $\mathrm{Li}_{2} \mathrm{IrO}_{3}$ are spin-orbit Mott insulator with fluctuating $S_{\text {eff }}=1 / 2$ moment above an antiferromagnetic $(\mathrm{AF})$ ordering around $15 \mathrm{~K}$, but neutron scattering study confirmed that both of them have very distinct magnetic structure which can not be explained by simple Heisenberg-Kitaev model as expected. Puzzle lies beneath the secret of their complicated magnetic exchange interactions. In order to understand these interactions in more details, three different kind of doping have been done.

- Iso-eletronic doping at $\mathrm{A}$ site of $\mathrm{A}_{2} \mathrm{IrO}_{3}:\left(\mathbf{N a}_{1-x} \mathbf{L i}_{x}\right)_{2} \mathbf{I r O}_{3}$

- Magnetic impurity doping at magnetic Ir site.

- Systematic nonmagnetic dilution at Ir site: $\mathbf{N a}_{2}\left(\mathbf{I r}_{1-x} \mathbf{T i}_{x}\right) \mathbf{O}_{3}$ and $\mathbf{L i}_{2}\left(\mathbf{I r}_{1-x} \mathbf{T i}_{x}\right) \mathbf{O}_{3}$

\subsection{Isoelectronic doping at $\mathrm{A}$ site: $\left(\mathrm{Na}_{1-x} \mathrm{Li}_{x}\right)_{2} \mathrm{IrO}_{3}$}

${ }^{1}$ Two end members of the $\left(\mathrm{Na}_{1-x} \mathrm{Li}_{x}\right)_{2} \mathrm{IrO}_{3}$ phase diagram exhibit very different magnetic structure despite different similarities in the structural, electronic and magnetic properties. One can expect a continuous change of magnetic properties and magnetic structure between $x=0$ and $x=1$. So far no $\mathrm{Li}_{2} \mathrm{IrO}_{3}$ single crystal is grown hence only way to study this

\footnotetext{
${ }^{1}$ In this doping study, experimental investigation crystal growth, powder XRD, scanning electron microscopy (SEM), thermodynamic and magnetic measurement was done by myself and single crystal XRD was done by S. K. Choi and Radu Coldea. Theoretical investigation was done by the group of Roser Valentí and I. I. Mazin. Detailed investigation of the $\left(\mathrm{Na}_{1-x} \mathrm{Li}_{x}\right)_{2} \mathrm{IrO}_{3}$ phase diagram is published in Ref. [74]. Experimental part of this section on $\left(\mathrm{Na}_{1-x} \mathrm{Li}_{x}\right)_{2} \mathrm{IrO}_{3}$ has mainly be taken from Ref. [74] which has been written by myself and corrected by my co-authors. Theoretical part described in this section is summarized from Ref. [74].
} 
systematic change on single crystals is to map out $\left(\mathrm{Na}_{1-x} \mathrm{Li}_{x}\right)_{2} \mathrm{IrO}_{3}$ phase diagram upto maximum Li-substitution possible. $\mathrm{Li}$ is smaller than $\mathrm{Na}$, hence gradual substitution of $\mathrm{Na}$ by Li will also systematically change the Ir-honeycomb structure. It can create a strain in Ir-honeycomb lattice and change shape of $\mathrm{IrO}_{6}$ octahedra and consequently trigonal crystal field. This may lead to change in the important electronic properties. A theoretical study on the honeycomb lattice iridates predicted that by changing this trigonal crystal field and the nearest neighbor hopping a topological state can be realized.[75]

\subsubsection{Experimental details}

Single crystals of $\left(\mathrm{Na}_{1-x} \mathrm{Li}_{x}\right)_{2} \mathrm{IrO}_{3}$ were grown using a similar procedure as previously used for $\mathrm{Na}_{2} \mathrm{IrO}_{3}$ [52]. A first calcination process was done at $750^{\circ} \mathrm{C}$ with stoichiometric proportions of carbonates $\left(\mathrm{Na}_{2} \mathrm{CO}_{3}\right.$ and $\left.\mathrm{Li}_{2} \mathrm{CO}_{3}\right)$ and $\mathrm{Ir}$ metal. After prereaction at $900^{\circ} \mathrm{C}$ the polycrystalline material was processed for crystal growth with excess $\mathrm{IrO}_{2}$ flux. The amount of excess $\mathrm{IrO}_{2}$ and the temperature of crystal growth were varied for different doping levels. Since with increasing Li content the solubility of the phase in the flux decreases, it is important to control both temperature and excess $\mathrm{IrO}_{2}$ for obtaining large enough crystals for bulk measurements.

Table 5.1: Comparison between the nominal and actual Li content determined by ICPMS in \% of $\mathrm{Li}$ in $\left(\mathrm{Na}_{1-x} \mathrm{Li}_{x}\right)_{2} \mathrm{IrO}_{3}$, reprinted from [74]

\begin{tabular}{ccc}
\hline \hline $\mathrm{x}$ & Nominal Li (\%) & ICPMS Li $(\%)$ \\
\hline 0.05 & 5 & $3.83( \pm 0.2)$ \\
0.1 & 10 & $9.5( \pm 0.5)$ \\
0.2 & 20 & $21.8( \pm 1.5)$ \\
0.3 & 30 & $33.2( \pm 1.1)$ \\
0.4 & 40 & $47.0( \pm 0.9)$ \\
\hline
\end{tabular}

The Na:Li ratio was determined by inductively coupled plasma mass spectrometry (ICPMS) on different pieces of crystals of every doping level. It is not possible to detect $\mathrm{Li}$ by an energy dispersive X-ray (EDX) analysis since $\mathrm{Li}$ is a light metal. In EDX one can only observe changes in the $\mathrm{Na}$ to Ir ratio, which decreases with Li doping. Table 5.1 gives a comparison between the nominal (starting composition) and the measured Li fractions. Some of the plate-like crystals were crushed and powder x-ray diffraction (XRD) was performed for the scattering angle range $10^{\circ} \leq 2 \theta \leq 100^{\circ}$ with $\mathrm{Cu} \mathrm{K}_{\alpha}$ radiation to estimate the change of the lattice parameters with Li doping. Single crystal x-ray diffraction (XRD) was performed using a Mo-source Oxford Diffraction Supernova diffractometer on crystals of $\left(\mathrm{Na}_{1-x} \mathrm{Li}_{x}\right)_{2} \mathrm{IrO}_{3}$ with nominal doping $x$ from 0.05 to 0.4 in order to obtain 


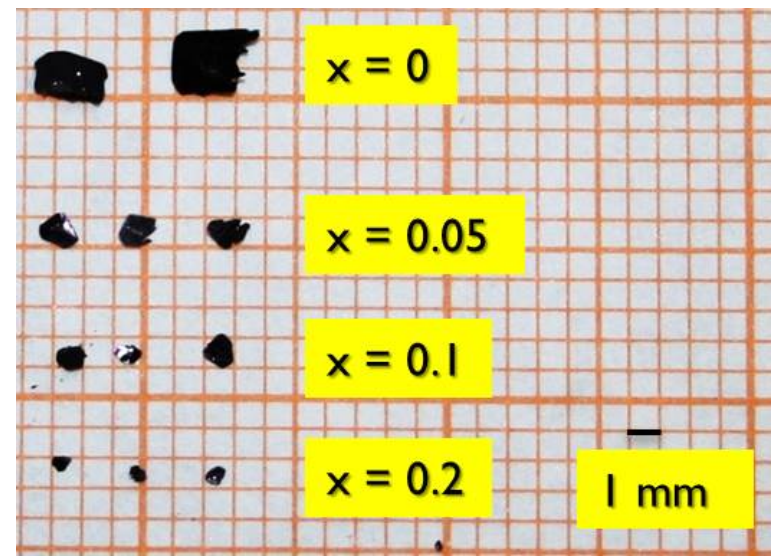

Figure 5.1:

$\left(\mathrm{Na}_{1-x} \mathrm{Li}_{x}\right)_{2} \mathrm{IrO}_{3}$ crys-

tals, decreasing in

size with increasing

Li doping $(x)$

lattice parameters and confirm the crystal structure and internal atomic coordinates. The samples were thin, plate-like crystals with a typical size of $70 \times 60 \times 10 \mu \mathrm{m}^{3}$. Magnetization, ac susceptibility and specific heat were measured in commercial SQUID magnetometer and physical property measurements systems, respectively.

Since the size of Li-doped crystals decreases with doping (Fig. 5.1), lumps of crystals were used for magnetization and specific heat measurements. Crystals (or lumps) were separated mechanically. Sometimes some remaining flux was present in the lump which gave a low temperature Curie tail in the $\chi(T)$ measurement.

\subsubsection{Theoretical calculations}

To determine the most stable and realistic structure for different dopings in $\left(\mathrm{Na}_{1-x} \mathrm{Li}_{x}\right)_{2} \mathrm{IrO}_{3}$ structural relaxation calculations were done on supercells of $\left(\mathrm{Na}_{1-x} \mathrm{Li}_{x}\right)_{2} \mathrm{IrO}_{3}$ for $0 \leq x \leq 1$ by density functional theory (DFT) by our theoretical collaborators I. I. Mazin and the group of Roser Valentí. Details of the calculations are given in Ref. [74]. They considered both aspects, firstly, taking experimental lattice parameters (Fig. refXRDc) and relaxing internal lattice parameters, secondly, full relaxation including atomic and lattice parameters.

In both cases, they observed for $0 \leq x \leq 0.25$ the energetically most favorable location for Li ions are Na positions at the center the honeycomb. In Fig. 5.2 the most stable crystal structure of $\left(\mathrm{Na}_{1-x} \mathrm{Li}_{x}\right)_{2} \mathrm{IrO}_{3}$ for a doping level of $x=0.25$ is shown. For $x>0.25 \mathrm{Li}$ goes to $\mathrm{Na}_{3}$ layer after substituting $\mathrm{Na}$ position in honeycomb layer. They also found that most stable structure for $x>0.25$ showed clustering of $\mathrm{Li}$ in the $\mathrm{Na}_{3}$ layer. 

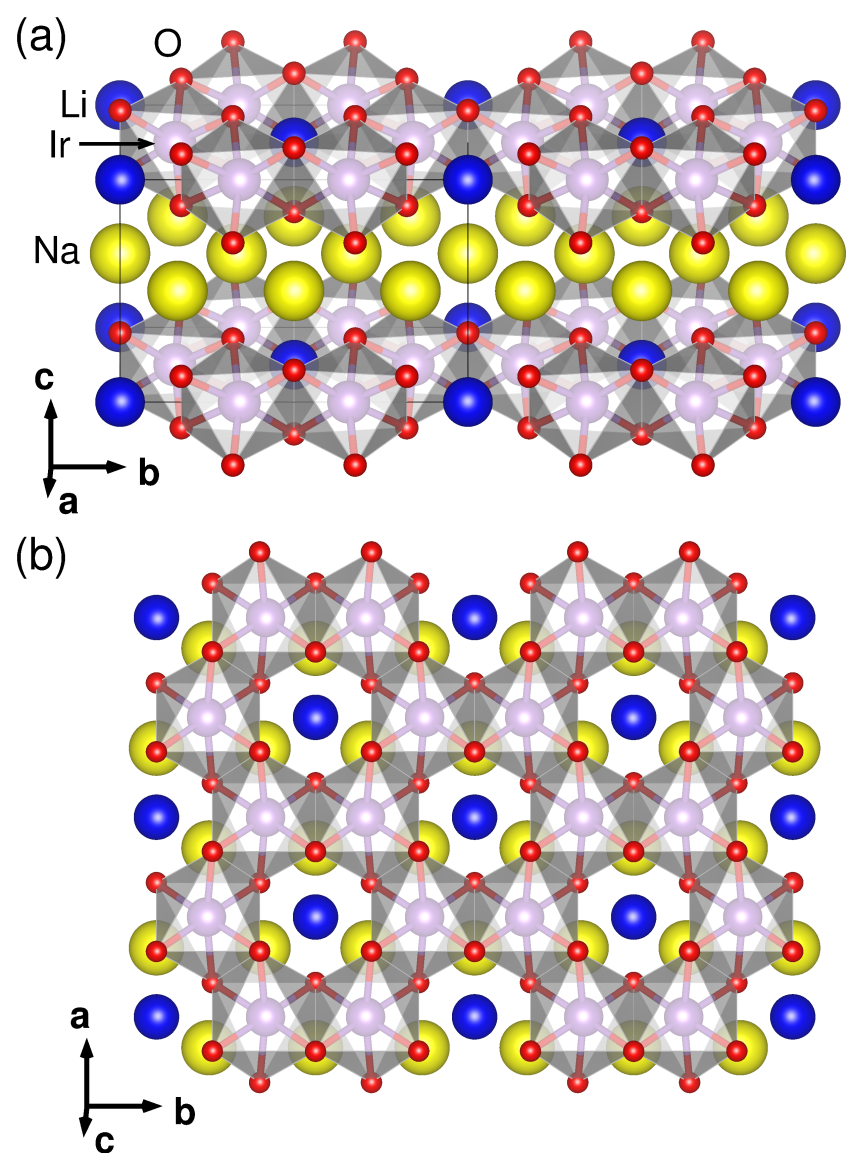

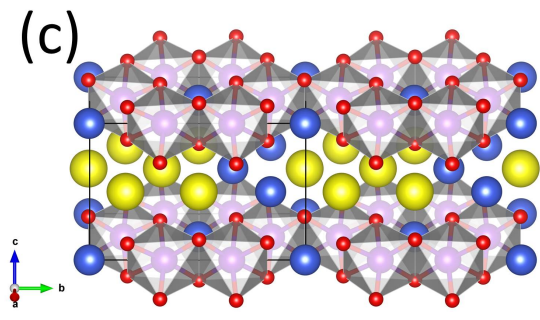

Figure 5.2: $\quad$ Calculated crystal structure of $\left(\mathrm{Na}_{1-x} \mathrm{Li}_{x}\right)_{2} \mathrm{IrO}_{3}$ for $x=0.25$ : (a) layered structure of $\mathrm{Ir}_{2} \mathrm{Li}$ and $\mathrm{Na}_{3}$ planes and (b) view on the $\operatorname{Ir}_{2} \mathrm{Li}$ planes, where the Ir atoms form a honeycomb lattice (c) Calculated crystal structure of $\left(\mathrm{Na}_{1-x} \mathrm{Li}_{x}\right)_{2} \mathrm{IrO}_{3}$ for $x=0.50$,layered structure of $\mathrm{Ir}_{2} \mathrm{Li}$ and $\mathrm{Na}_{2} \mathrm{Li}$ planes. Reprinted from [74]

\subsubsection{Results and Discussion}

\subsubsection{Low doping $(x<0.25)$}

Structural changes: Powder XRD of crushed $\left(\mathrm{Na}_{1-x} \mathrm{Li}_{x}\right)_{2} \mathrm{IrO}_{3}$ crystals shows single phase crystals up to $x=0.2$ (see Fig. 5.3(b)). These crystals are very plate-like and only (00n) peaks could be observed. Moreover, while ICPMS confirms the inclusion of Li (see Table 5.1) at the concentration $x=0.2$, there is almost no shift of the (001) peak, implying almost no change in the $c$ lattice parameter for the range $0 \leq x \leq 0.2$.

The lattice parameters as a function of doping were determined by single crystal XRD by our collaborator Radu Coldea et. al. Complete diffraction patterns for structural refinement were collected for the best samples at each doping. Nevertheless a few challenges were facced when refining the diffraction pattern of the Li-doped samples. Namely, Li scatters x-rays very weakly and its precise position in the structure cannot be uniquely determined from $\mathrm{x}$ ray measurements alone, especially at low Li concentrations and in the presence of dominant scatterers like Ir (with 77 electrons), refinements of the crystal structure with Li in different 

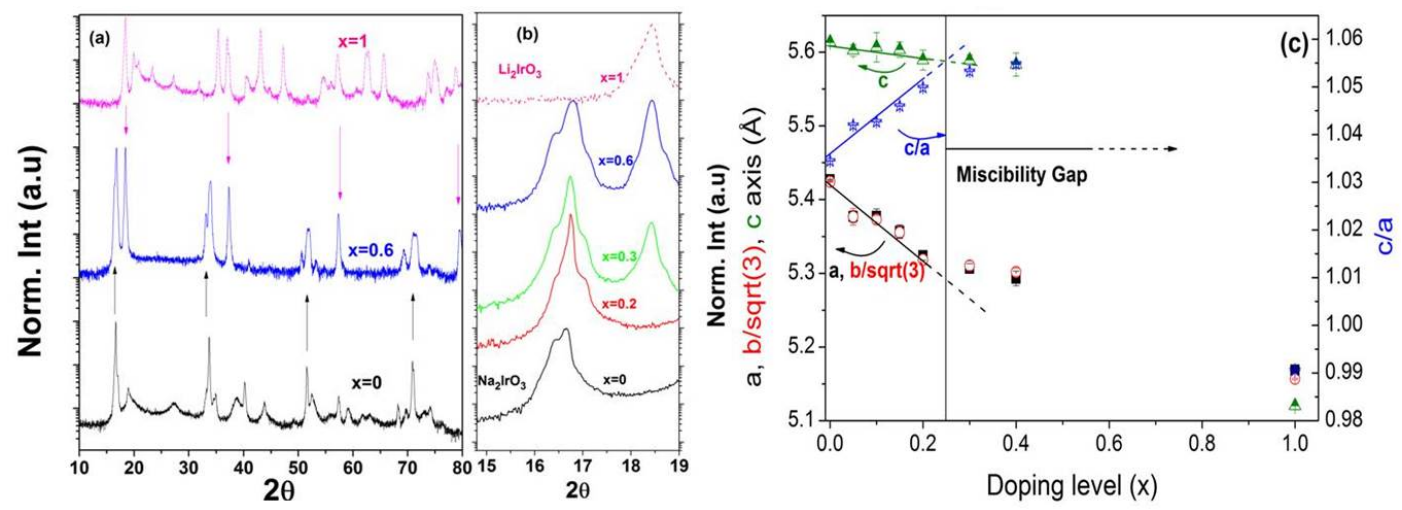

Figure 5.3: (a) Powder XRD of the crushed $\left(\mathrm{Na}_{1-x} \mathrm{Li}_{x}\right)_{2} \mathrm{IrO}_{3}$ crystals for $\mathrm{x}=0,0.6$ and $\mathrm{x}=1$. The magenta colored downward arrows point $\mathrm{x}=0.6 \mathrm{XRD}$ peaks that matches with $\mathrm{x}=1(00 \mathrm{n})$ peaks and black colored upward arrows point $\mathrm{x}=0.6 \mathrm{XRD}$ peaks that matches with $\mathrm{x}=0(00 \mathrm{n})$ peaks. (b) Zoomed XRD spectra in the $2 \theta$ region 15 to $19{ }^{\circ}$ for all values of $\mathrm{x}$. (c) Lattice parameters obtained from single crystal XRD of $\left(\mathrm{Na}_{1-x} \mathrm{Li}_{x}\right)_{2} \mathrm{IrO}_{3}$ single crystals $(x=1$ obtained from Ref. [60]). The horizontal arrow marks the miscibility gap region where samples showed phase separation. Solid straight lines (extended by dashed lines in the miscibility gap region) are guides to the eye. Reprinted from [74]

$\mathrm{Na}$ positions (in the honeycomb $\mathrm{Ir}_{2} \mathrm{Na}$ layer and in the hexagonal $\mathrm{Na}_{3}$ layer) gave rather similar results. Since structural relaxation calculations (see previous section) suggest a strong energetic preference for the doped Li to replace the Na in the Ir honeycomb layers (for $x \leq 0.25$ ), the final structural refinement was performed assuming that Li randomly replaces $\mathrm{Na}$ at this site [74]. The refinement converged well only when some finite degree of site mixing $(f>0)$ was assumed also on the nominally Ir honeycomb site, so that the occupation at this site was assumed to be $(1-f) \operatorname{Ir}+f \mathrm{Na}$. In order to preserve the total atomic count the honeycomb center site occupation was assumed to be $4 x \mathrm{Li}+(1-4 x-2 f) \mathrm{Na}+2 f \mathrm{Ir}$. The refined atomic positions for $x=0.05$ is listed in Table 5.2.

In order to determine the lattice parameters accurately for each doping between 10 to 20 samples were measured and the obtained average values are plotted in Fig. 5.3(c) with the error bars indicating the spread of values for each nominal composition. Throughout the range $0.05 \leq x \leq 0.2$, the diffraction patterns show sharp peaks that could be well indexed and refined with a $C 2 / m$ crystal structure derived from the undoped $(x=0)$ parent $\mathrm{Na}_{2} \mathrm{IrO}_{3}$ in Ref. [36]. For lower dopings $x=0.05,0.1$ the diffraction patterns were consistently indexed in terms of a single crystal (no twins). For dopings $x=0.15,0.2$, samples showed two or three co-existing twins and in this case refinement was successfully performed using multitwin techniques with the same unit cell parameters and crystal structure for all co-existing twins. Throughout the range $0.05 \leq x \leq 0.2$ the $C 2 / m$ crystal structure of parent $\mathrm{Na}_{2} \mathrm{IrO}_{3}$ provides a good description of the observed diffraction pattern, confirming single-phase 
Table 5.2: Structural parameters for $x=0.05$ Li-doping from single-crystal x-ray data at $300 \mathrm{~K}$. (C2/m space group, $a=5.379(5) \AA, b=9.314(5) \AA, c=5.594(5) \AA, \beta=108.714(5)^{\circ}$, $Z=4)$. $U$ is the isotropic displacement. The goodness-of-fit(S) was $1.269, w_{R 2}=0.1684$, $R_{1}=0.0632\left(R_{\text {int }}=0.0797, R_{\sigma}=0.051\right)$. Reprinted from [74]

\begin{tabular}{lllllll}
\hline \hline Atom & Site & $x$ & $y$ & $z$ & Occ & $U\left(\AA^{2}\right)$ \\
\hline Ir1 & $4 g$ & 0.5 & $0.1667(1)$ & 0 & 0.849 & $0.0074(6)$ \\
Na1 & $4 g$ & 0.5 & $0.1667(1)$ & 0 & 0.151 & $0.0074(6)$ \\
Na2 & $2 a$ & 0 & 0 & 0 & 0.498 & $0.0092(8)$ \\
$\mathrm{Ir} 2$ & $2 a$ & 0 & 0 & 0 & 0.302 & $0.0092(8)$ \\
$\mathrm{L} 22$ & $2 a$ & 0 & 0 & 0 & 0.2 & $0.0092(8)$ \\
$\mathrm{Na} 3$ & $2 d$ & 0.5 & 0 & 0.5 & 1 & $0.021(4)$ \\
$\mathrm{Na} 4$ & $4 h$ & 0.5 & $0.3388(11)$ & 0.5 & 1 & $0.019(3)$ \\
$\mathrm{O} 1$ & $8 j$ & $0.758(3)$ & $0.1732(11)$ & $0.792(3)$ & 1 & $0.013(3)$ \\
$\mathrm{O} 2$ & $4 i$ & $0.720(4)$ & 0 & $0.210(4)$ & 1 & $0.013(4)$ \\
\hline \hline
\end{tabular}

crystals with this structure. Both the $a$ and $b$ lattice parameters strongly decrease at the same rate with increasing doping $(b / \sqrt{3} \simeq a$, which confirms a globally almost undistorted honeycomb Ir structure in the low Li doped region) while the $c$ parameter remains almost constant (Fig. 5.3(c)). Remarkably, the $c / a$ ratio increases with increasing doping $x$ up to 0.2 (Fig. 5.3(c)) while it is reduced by $5 \%$ in fully-doped $(x=1) \mathrm{Li}_{2} \mathrm{IrO}_{3}$ compared to the undoped $(x=0) \mathrm{Na}_{2} \mathrm{IrO}_{3}$. Hence there is no effective $c$-axis pressure in the low Li doping region.

Magnetic Susceptibility: In Fig. 5.4 the temperature $T$ dependence of the magnetic susceptibility $\chi(T)=M / H$ is shown for $\left(\mathrm{Na}_{1-x} \mathrm{Li}_{x}\right)_{2} \mathrm{IrO}_{3}$ for dopings $x=0.05$ to 0.2 measured at $H=1 T$ between 2 and $300 \mathrm{~K}$. The inverse susceptibility $\left(\chi^{-1}\right)$ (not shown) and susceptibility $(\chi)$ were fitted to the Curie-Weiss (CW) law given in Eqn. 4.7 (red lines in Fig. 5.4) between 150 and $300 \mathrm{~K}$. For all $x$ values measured, $\chi_{0} \approx 10^{-4} \mathrm{~cm}^{3} / \mathrm{mol}$ and $C=0.4$ $0.5 \mathrm{~cm}^{3} \mathrm{~K} / \mathrm{mol}$, while the Weiss temperature $\left(\theta_{W}\right)$ is dependent on doping (see Fig. 5.26(b)). Since for single crystalline $\mathrm{Na}_{2} \mathrm{IrO}_{3}$ an anisotropic susceptibility was observed, [52] it is expected a certain anisotropy in the different Li-substituted single crystals as well. The susceptibility measured on lumps of arbitrary oriented crystals is therefore different from the average between $\chi_{a}$ and $\chi_{c}$ and would not match a perfectly random polycrystalline sample. This explains a $\approx 20 \%$ variation in the $C$ parameter of the Curie-Weiss fit for the different Li substituted samples.

$\chi(\mathrm{T})$ shows a kink for all measured $x$ (marked with arrows in Fig. 5.4) indicating long range AF ordering. No spin glass freezing has been observed, as confirmed by FC-ZFC and 


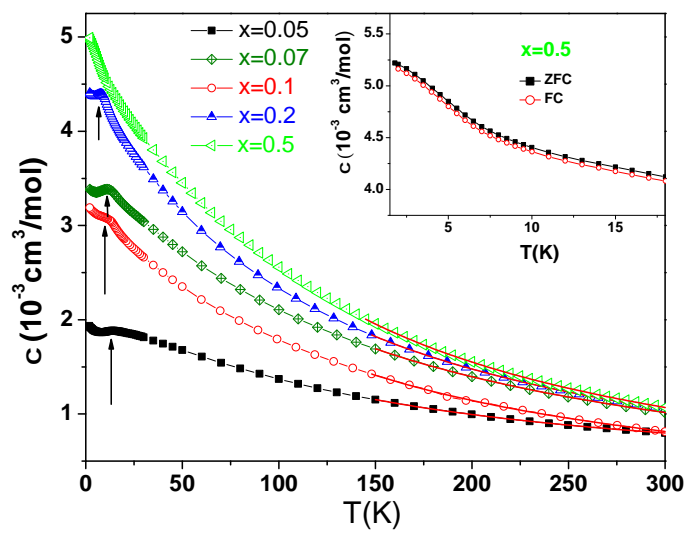

Figure 5.4: (Color online) Magnetic susceptibility $\chi(T)$ vs $\mathrm{T}$ for $\mathrm{x}=0.05$ to 0.2 and $\mathrm{x}=0.5$. The red line indicates fitting by $\mathrm{CW}$ behavior (Eqn. 4.7). The arrows mark the positions of $T_{N}$. FC and ZFC measurements for $x=0.5$ are shown in the inset. Reprinted from [74]

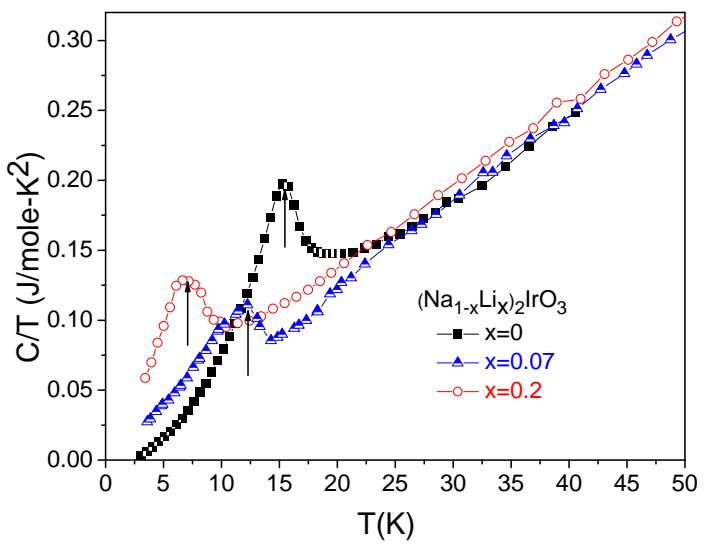

Figure 5.5: (Color online) Heat capacity as $C(T) / T$ of single phase $\left(\mathrm{Na}_{1-x} \mathrm{Li}_{x}\right)_{2} \mathrm{IrO}_{3}$ crystals. The arrows mark the positions of $T_{N}$. Reprinted from [74]

ac susceptibility measurements. The position of maxima was determined by plotting $\frac{d \chi}{d T} \mathrm{vs}$ $\mathrm{T}$ where the zero crossing is assigned to the AF transition temperature $T_{N}$.

Heat Capacity: Fig. 5.5 shows the $\left(\mathrm{Na}_{1-x} \mathrm{Li}_{x}\right)_{2} \mathrm{IrO}_{3}$ heat capacity divided by temperature $(C / T)$ measurements for crystals up to $x=0.2$. These measurements confirm bulk $\mathrm{AF}$ ordering and the extracted $T_{N}$ (from the onset of the lambda-like peaks in $C / T$ ) as a function of Li doping agrees with the values from the susceptibility measurements. In order to obtain information on the size of the ordered moment, the magnetic entropy was determined from integration of the magnetic heat capacity $(\Delta C(T) / T)$. The latter was calculated by subtracting the phonon contribution. For $x=0$ the phonon heat capacity was obtained from the non-magnetic reference $\mathrm{Na}_{2} \mathrm{SnO}_{3}$ while for $x=0.280 \%$ contribution of $\mathrm{Na}_{2} \mathrm{SnO}_{3}$ and $20 \%$ of $\mathrm{Li}_{2} \mathrm{SnO}_{3}$ was used as reference. Integration of $\Delta C / T$ vs. $T$ revealed that values of the magnetic entropy $\Delta S=0.2 R \ln 2$ and $0.12 \mathrm{R} \ln 2$ at $\mathrm{T}_{N}$ for $x=0$ and $x=0.2$, respectively. This suggested a suppression of the ordered moment by Li substitution, which may be due to stronger frustration and/or local lattice distortions that affect the magnetic exchanges.

\subsubsection{Higher doping $(x>0.25)$}

The systematic suppression of $T_{N}$ with increasing $x$ for $\left(\mathrm{Na}_{1-x} \mathrm{Li}_{x}\right)_{2} \mathrm{IrO}_{3}$ crystals up to $x=0.2$ suggests the possibility of a magnetic quantum phase transition at larger $x$. However, 
for larger Li content, i.e., from $x=0.25$ to $x=0.6$ a clear indication of phase separation was observed in the respective samples. The powder XRD patterns of crushed crystals are shown in Fig. 5.3(a,b). Fig. 5.3(a) shows that the $\mathrm{x}=0.6$ pattern contains $(00 \mathrm{n})$ peaks located close to both pure $\mathrm{Li}_{2} \mathrm{IrO}_{3}$ (marked by downwards pointing magenta colored arrows) and $\mathrm{Na}_{2} \mathrm{IrO}_{3}$ (indicated by upwards pointing black colored arrows). A closer inspection of the region near (001) with more different compositions is given in Fig. 5.3(b). It shows that for all nominal compositions larger than 0.2 two phases are observed, one close to $\mathrm{x}=0.2$, the other one $\mathrm{x}=1$. In the single crystal XRD at the higher dopings $x=0.3,0.4$ the samples showed many co-existing single crystal grains compared to the crystals at dopings $x \leq 0.2$ region and the diffraction data could not be consistently indexed by the same unit cell parameters for all co-existing grains, suggesting that the samples were not single-phase, but possibly a mixture of phases with different lattice parameters.

The two phase scenario was further supported by the results of scanning electron microscopy (SEM) shown in Fig. 5.6 (a) and (b) for $x=0.3$ and 0.6 crystals, respectively. For $x=0.3$ two phases were observed. On the lighter contrast lines (marked by arrows in Fig. 5.6(a)) EDX shows a much lower ratio of Na:Ir (almost only Ir). Hence this lighter contrast can be attributed to the $\mathrm{Li}_{2} \mathrm{IrO}_{3}$ phase. For $x=0.6$ hexagonal shaped micro-domains appear (average size 2-3 $\mu \mathrm{m}$ ). The SEM picture was taken after cleaving the crystals and micro-domains of the same size are still present. EDX measurements showed a very small $\mathrm{Na}$ :Ir ratio at the domain boundaries, indicating also $\mathrm{Li}_{2} \mathrm{IrO}_{3}$ micro-domains. In fact ICPMS indicates (Table 5.1) an increase in Li content for $x \geq 0.3$, although there is not much change in the lattice parameters for $x=0.3$ and 0.4 compared to $x=0.2$ (see Fig. 5.3(c)). The trend of change in lattice parameters significantly deviates after $\mathrm{x}=0.25$. This confirms that in the region $0.25<x \leq 0.6 \mathrm{Li}$ is not incorporated into the main $\left(\mathrm{Na}_{1-x} \mathrm{Li}_{x}\right)_{2} \mathrm{IrO}_{3}$ phase but rather forms separate micro-domains of $\mathrm{Li}_{2} \mathrm{IrO}_{3}$ indicating a miscibility gap in the phase (see Fig. 5.26(b)).

This is further confirmed when heat capacity is measured for $0.25 \leq x \leq 0.6$. We observe in this whole range a smeared lambda-like peak at $5.5 \mathrm{~K}$ (Fig. 5.7), which implies that $T_{N}$ does not depend on doping in this entire range. This means that the magnetic contribution originates from the main $\mathrm{Na}_{3} \mathrm{Ir}_{2} \mathrm{LiO}_{6}$ phase, which is not affected by further doping. The micro-domains of $\mathrm{Li}_{2} \mathrm{IrO}_{3}$ apparently do not exhibit long range order, presumably due to structural disorder. [60] For $\mathrm{x}=0.5$ magnetic susceptibility neither shows conventional antiferromagnetic (AF) ordering (Fig. 5.4) nor any separation between ZFC-FC susceptibility(inset) indicative of spin-glass behavior. $\mathrm{SO}$ we speculate that for this high doping region the presence of a multidomain $\mathrm{Li}_{2} \mathrm{IrO}_{3}$ phase smears out any AF transition in susceptibility. 


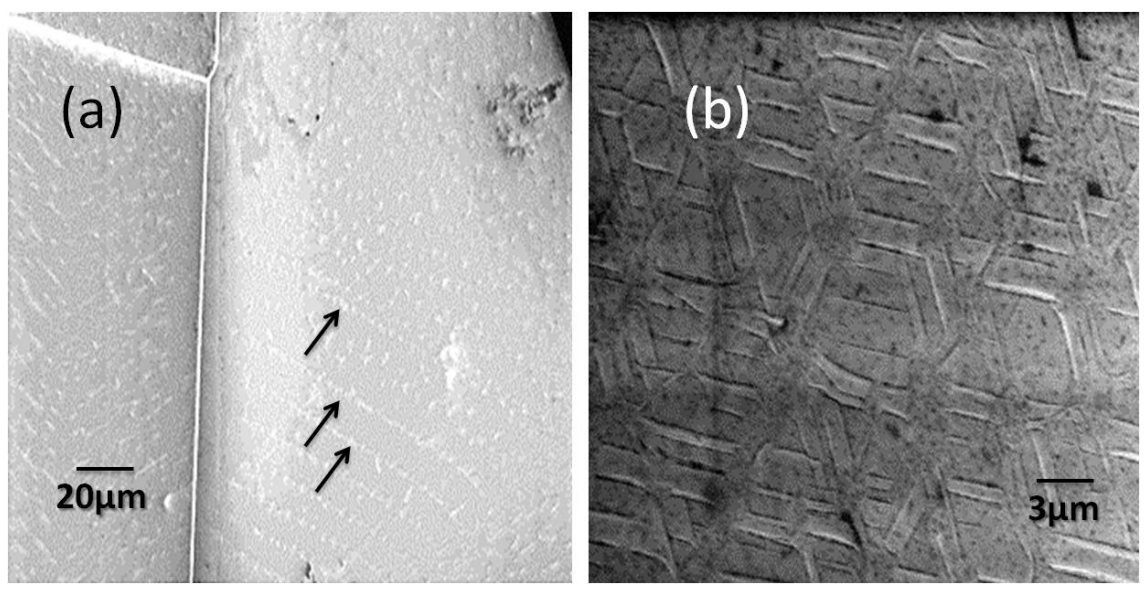

Figure 5.6: SEM picture of (a) $x=0.3$ and (b) $x=0.6\left(\mathrm{Na}_{1-x} \mathrm{Li}_{x}\right)_{2} \mathrm{IrO}_{3}$ crystals. Reprinted from [74]

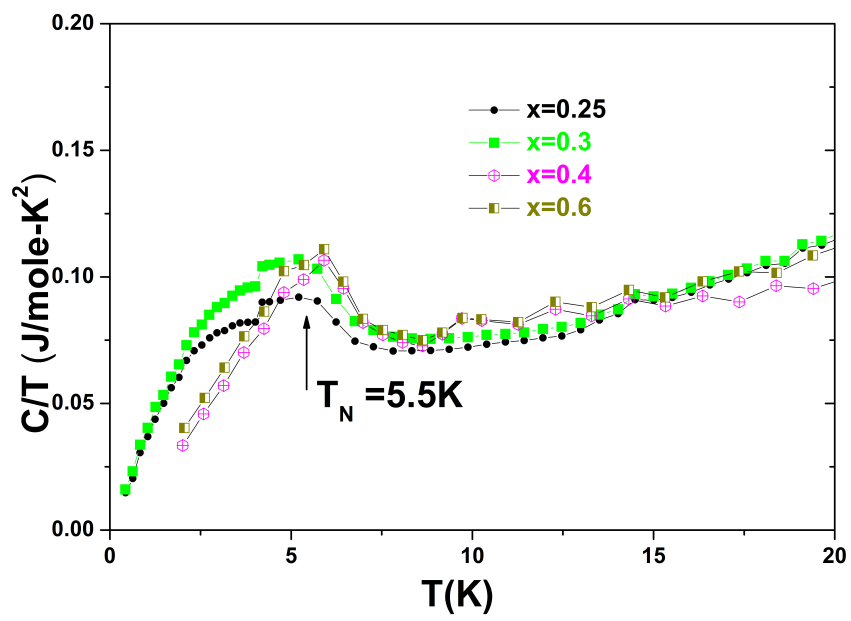

Figure 5.7: Heat capacity as $C(T) / T$ of multiphase $\mathrm{x} \geq$ 0.25. $\left(\mathrm{Na}_{1-x} \mathrm{Li}_{x}\right)_{2} \mathrm{IrO}_{3}$ crystals. The arrows mark the positions of $T_{N}$ which is fixed with increasing $\mathrm{x}$. Reprinted from $[74]$

DFT supercell calculations of $\left(\mathrm{Na}_{1-x} \mathrm{Li}_{x}\right)_{2} \mathrm{IrO}_{3}$ at various dopings (see section 5.1.2) show that in the $0<x<0.25$ range, $\mathrm{x}=0.125$ and $\mathrm{x}=0.25$ results are compatible with a uniform phase within the computational accuracy. However, after the $\operatorname{Ir}_{2} \mathrm{Na}$ planes are completely substituted by Li, further doping $(x>0.25)$ is energetically unfavorable: for $0.25<x<1$ the energies of the lowest uniform phases are at least about $30 \mathrm{meV} / \mathrm{Ir}$ higher than those of the separated phases (Not shown here) [74]. Moreover, the lowest-energy solutions tend to clusterize on the scale allowed by a given supercell. The inclusion of spinorbit coupling, a Hubbard $U=3 \mathrm{eV}$ and magnetism [74] leads to an even more pronounced instability towards phase separation ( $\gtrsim 40 \mathrm{meV} / \mathrm{Ir}$ ), as shown in Fig. 5.26(a), where the straight line indicates the energy of the corresponding mixture of separated phases. 


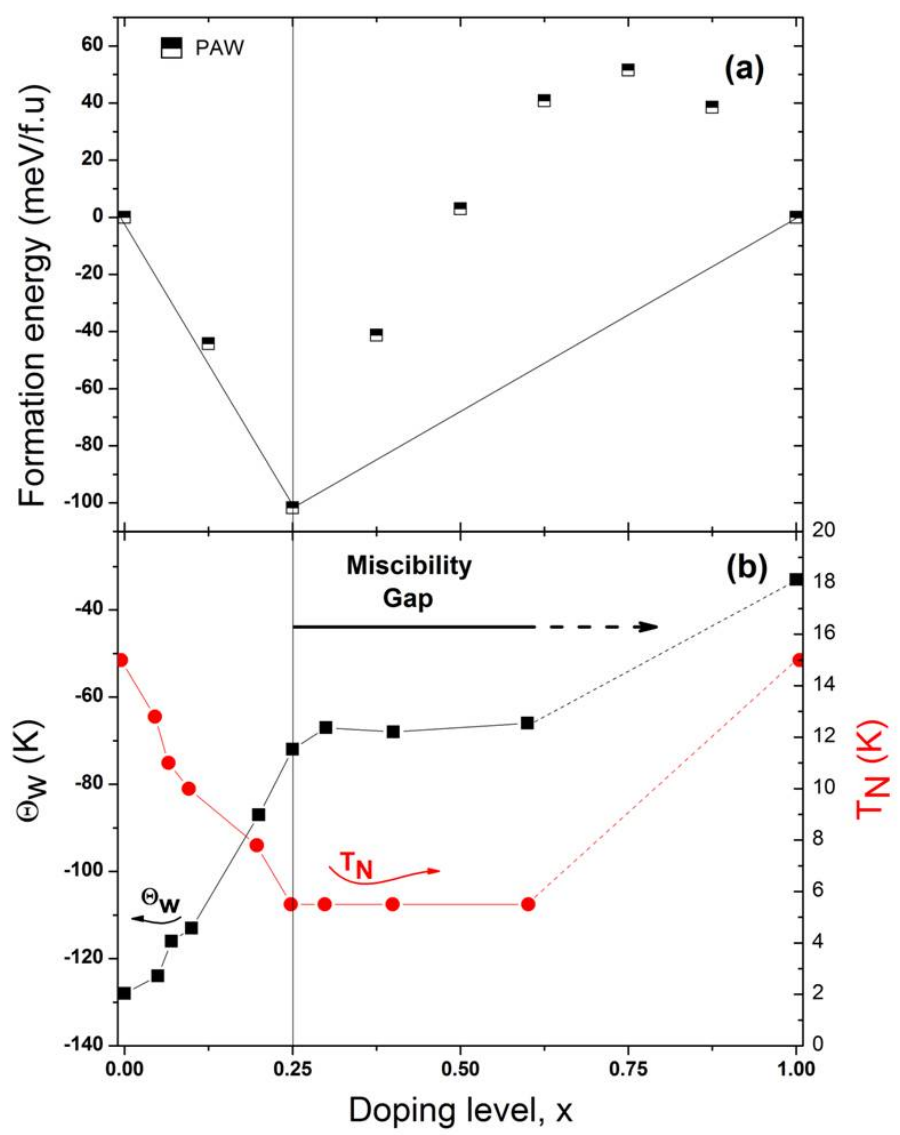

Figure 5.8: (Color online) (a) Phase diagram of $\left(\mathrm{Na}_{1-x} \mathrm{Li}_{x}\right)_{2} \mathrm{IrO}_{3}$ obtained from spin-polarized $\mathrm{GGA}+\mathrm{SOC}+\mathrm{U}$ total energy calculations. Shown are the formation energies obtained with the PAW basis. The vertical line indicates the composition at $x=0.25$ which is very stable $\mathrm{Na}_{3} \mathrm{LiIr}_{2} \mathrm{O}_{6}$ structure with alternating $\mathrm{LiIr}_{2} \mathrm{O}_{6}$ and $\mathrm{Na}_{3}$ layers. (b) Phase diagram with $T_{N}$ and CW temperature $\theta_{W}$ of $\left(\mathrm{Na}_{1-x} \mathrm{Li}_{x}\right)_{2} \mathrm{IrO}_{3}$, data at $x=1$ are from Ref. [60]. The miscibility gap region is indicated by the horizontal arrow. Reprinted from [74]

\subsubsection{Conclusions}

Based on structural, thermodynamic, SEM and magnetic measurements first principles calculations, we propose the following scenario: in the $\left(\mathrm{Na}_{1-x} \mathrm{Li}_{x}\right)_{2} \mathrm{IrO}_{3}$ system a miscibility gap emerges for $x>0.25$ (Fig. 5.26). The stable structure in this region shows a phase separation into an ordered $\mathrm{Na}_{3} \mathrm{Ir}_{2} \mathrm{LiO}_{6}$ phase, with alternating $\mathrm{LiIr}_{2} \mathrm{O}_{6}$ and $\mathrm{Na}_{3}$ planes, and a Li-rich phase very close in composition to $\mathrm{Li}_{2} \mathrm{IrO}_{3}$. As the crystal grows, the $\mathrm{Na}_{3} \mathrm{Ir}_{2} \mathrm{LiO}_{6}$ phase nucleates first, and forms the matrix. We suggest that nucleation for the $\mathrm{Li}_{2} \mathrm{IrO}_{3}$ phase should start at higher temperature but at the low temperature it nucleates around multiple centers of the matrix $\left(\mathrm{Na}_{1-x} \mathrm{Li}_{x}\right)_{2} \mathrm{IrO}_{3}$ phase, forming hexagonal micro-domains.

However, one can not completely exclude a possible high temperature solid-solution phase. One possibility could be that there may exist a critical temperature of the miscibility gap for each nominal composition $x \geq 0.25$ above which a metastable single phase exists and that temperature is above the crystal growth temperature, and therefore it becomes extremely hard to get single-phase single-crystals in this doping region. A similar work on $\left(\mathrm{Na}_{1-x} \mathrm{Li}_{x}\right)_{2} \mathrm{IrO}_{3}$ has claimed single-phase crystals for $x=0.7-0.9$ including a lowest $T_{N}$ 
$=1.5 \mathrm{~K}$ for $x=0.7$.[76] But our work inevitably proves existence of a miscibility gap for $x \geq 0.25$ and very likely extends beyond $x=0.6$ according to the energy calculation.

Hence the proposed lowest $T_{N}$ by Cao. et. al. may not be at $x=0.75$ but nearer to $x=1$. They have done their elemental analysis by EDX which could be the cause of the uncertainty in the determination of $\mathrm{Li}$ composition ( $x$-value). So in $\left(\mathrm{Na}_{1-x} \mathrm{Li}_{x}\right)_{2} \mathrm{IrO}_{3}$ phase diagram a continuous suppression of $T_{N}$ and strong change in $a$ and $b$ lattice parameter have been observed followed by a miscibility gap at higher doping. Strong change of inplane lattice parameter indicates significant change in electronic parameters in low doped $\left(\mathrm{Na}_{1-x} \mathrm{Li}_{x}\right)_{2} \mathrm{IrO}_{3}$ single crystals.

\subsubsection{Preliminary study of electronic property on $\left(\mathrm{Na}_{1-x} \mathrm{Li}_{x}\right)_{2} \mathrm{IrO}_{3}$ single crystals}

(a)

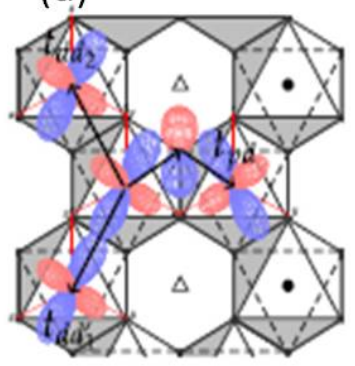

(b)

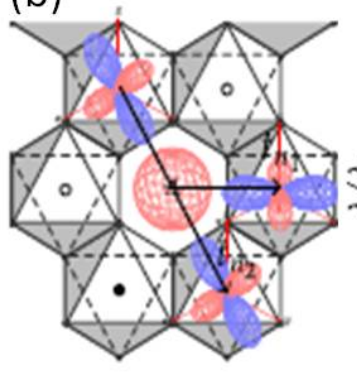

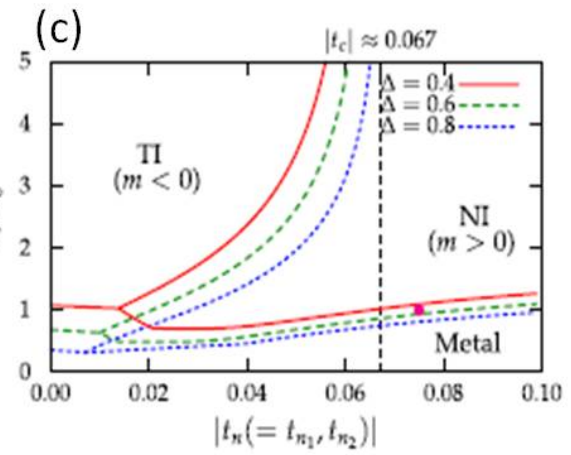

Figure 5.9: (a) and (b) Hopping parameters considered in the tight-binding model by C.H. Kim et.al. (c) Phase diagram as a function of $t_{n}$ and SOC for different value of trigonal crystal field $(\Delta)$. The pink dot indicates the position of $\mathrm{Na}_{2} \mathrm{IrO}_{3}$. Reprinted from [75]

C. H. Kim et al. determined tight-binding (TB) parameters for honeycomb lattice iridate $\mathrm{Na}_{2} \mathrm{IrO}_{3}$ by first principal calculations. [75] In Fig. 5.9 all the hopping parameters between Ir 5d orbitals are shown: two direct nearest neighbor hoppings $t_{d d_{1}}$ and $t_{d d_{2}}$; indirect hopping via Oxygen $2 p$ orbital $t_{p d} ; 2^{\text {nd }}$ and $3^{r d}$ nearest neighbor indierect hopping via $\mathrm{Na} 3 \mathrm{~s}$ orbital $t_{n 1}$ and $t_{n 2}$ respectively. Band structure calculation were done with these hopping parameters including the spin-orbit coupling (SOC, $\lambda$ ) and the trigonal crystal field $(\Delta)$. They claimed that the value of $t_{n}$ and $\Delta$ were very small in $\mathrm{Na}_{2} \mathrm{IrO}_{3}$ and a pure $J_{\text {eff }}=1 / 2$ spin-orbit Mott insulating state has been realized. In their calculation they showed that by varying $t_{n}$ a "band inversion" is possible in this system. The topological phase diagram as function of $t_{n}$ and SOC is shown in Fig. 5.9. For the certain values of $t_{n}$, the gap closes at the $M$ point in the Brillouin zone and an edge state is realized. At the end of their paper, 


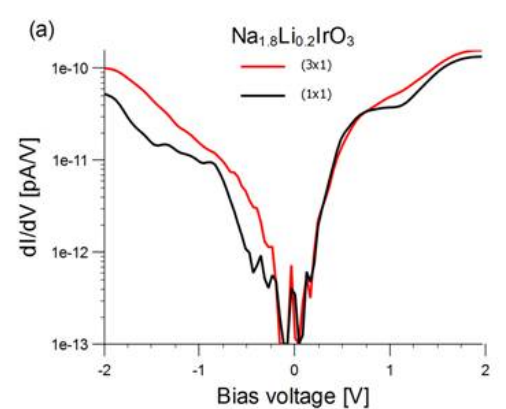

(c)

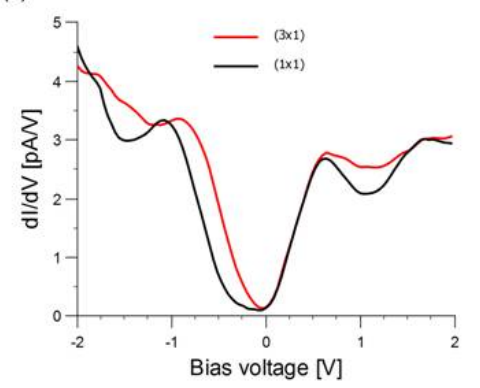

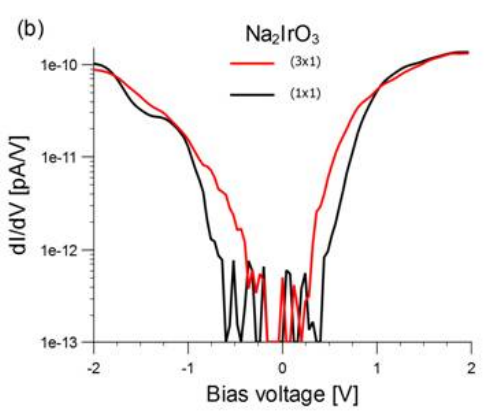

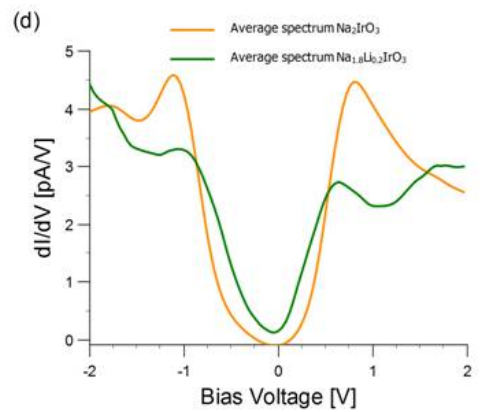

Figure 5.10: Logarithmic plot of the average spectra of the $(1 \times 1)$ and $(3 \times 1)$ surface of $x=0.1,\left(\mathrm{Na}_{1-x} \mathrm{Li}_{x}\right)_{2} \mathrm{IrO}_{3}$, (b) The corresponding spectra of $\mathrm{Na}_{2} \mathrm{IrO}_{3}$. (c) Normalized spectra of the $\left[\mathrm{Na}_{0.9} \mathrm{Li}_{0.1}\right]_{2} \mathrm{IrO}_{3}$ surface. (d) Direct comparison of the average spectra of the $x=0$ and $x=0.1,\left(\mathrm{Na}_{1-x} \mathrm{Li}_{x}\right)_{2} \mathrm{IrO}_{3}$ crystal surface assuming equal coverage of $(3 \times$ $1)$ and $(1 \times 1)$ termination, respectively. Reprinted from master thesis of F. Lüpke with permission. [77]

they have proposed that by controlling long range hopping $t_{n}$ and trigonal $\mathrm{CF} \Delta$ one can realize a topological state in $\mathrm{Na}_{2} \mathrm{IrO}_{3}$. They have suggested two ways: (i) epitaxial strain in honeycomb layer by Li substitution at $\mathrm{Na}$ position or (ii) increasing interlayer distance by intercalation of some atom in between two honeycomb layer. [75]

For $x \leq 0.25$ in $\left(\mathrm{Na}_{1-x} \mathrm{Li}_{x}\right)_{2} \mathrm{IrO}_{3}$ crystals, $a$ and $b$ lattice parameters show significant change which can introduce significant strain within honeycomb layer. Replacing the Naposition by Li reduces the Ir-honeycomb size and due to that, long range hopping parameters $t_{n}$ change also. The trigonal distortion of the $\mathrm{IrO}_{6}$ octahedra also change due to Li substitution. So one can expect significant changes in the band structure near Fermi energy at some $k$-points in reciprocal space.

Hence, to investigate the change in the electronic band structure, very preliminary studies were done on these $\left(\mathrm{Na}_{1-x} \mathrm{Li}_{x}\right)_{2} \mathrm{IrO}_{3}$ single crystals by Felix Lüpke in his master thesis $[77]^{2}$ using scanning tunneling spectroscopy (STS). Scanning tunneling microscopy (STM) at fixed bias voltage on cleaved surface of $x=0$ and $x=0.1,\left(\mathrm{Na}_{1-x} \mathrm{Li}_{x}\right)_{2} \operatorname{IrO}_{3}$ crystals

\footnotetext{
${ }^{2}$ Some of the texts in the subsection 5.1.5 and the STS figure (Fig. 5.10) are directly taken from master thesis of Felix Lüpke with the permission.
} 
showed atomic resolutions of the the surface. A detailed analysis showed that basically cleaved surfaces probed by STM tip were reconstructed surface of Ir-terminated layer, (3 $\times 1)$ surface and Na-terminated layer, $(1 \times 1)$ surface. [77] The scanning tunneling spectra of the two surfaces of $x=0.1 \mathrm{Li}$ substituted crystal and $\mathrm{Na}_{2} \mathrm{IrO}_{3}$ crystal are presented in Fig. 5.10 (a) and (b)respectively. The band gaps in Li substituted sample are for $(1 \times 1)$ surface $E_{g} \approx 0.75 \mathrm{eV}$ and for $(3 \times 1)$ surface $E_{g}^{\prime} \approx 0.3 \mathrm{eV}$. These values are smaller than the band gaps observed on the respective $\mathrm{Na}_{2} \mathrm{IrO}_{3}$ surfaces $\left(E_{g} \approx 1 \mathrm{eV}\right.$ and $\left.E_{g}^{\prime} \approx 0.5 \mathrm{eV}\right)$. Comparison of the normalized spectra for the two surfaces of $x=0.1$ crystals are shown in Fig. 5.10 (c). For the positive bias voltage both the spectra look similar but for the negative bias the peak closer to $E_{F}$ shifts to $V_{\text {bias }}=-0.9 \mathrm{eV}$ for $(3 \times 1)$ surface from $-1.1 \mathrm{eV}$ for $(1 \times 1)$ surface. A comparison of the normalized spectra of $\mathrm{Na}_{2} \mathrm{IrO}_{3}$ and $x=0.1\left(\mathrm{Na}_{1-x} \mathrm{Li}_{x}\right)_{2} \mathrm{IrO}_{3}$ is shown in Fig. 5.10 (d). Li substitution shifts the whole spectra more near to Fermi energy $\left(E_{F}\right)$ and introduces a reduction in band gap. This measured gap is not true charge gap. This effect of reduction by Li substitution needs to be investigated by optical spectroscopy for comparison of charge gap. No conducting edge states were observed in the Li substituted crystal for the STS measurement along the cleaving edges. Though a significant change in the band structure is hinted by the low $\mathrm{Li}$ substitution in the $\mathrm{Na}_{2} \mathrm{IrO}_{3}$ crystals.

\subsection{Magnetic impurity doping at Ir-site}
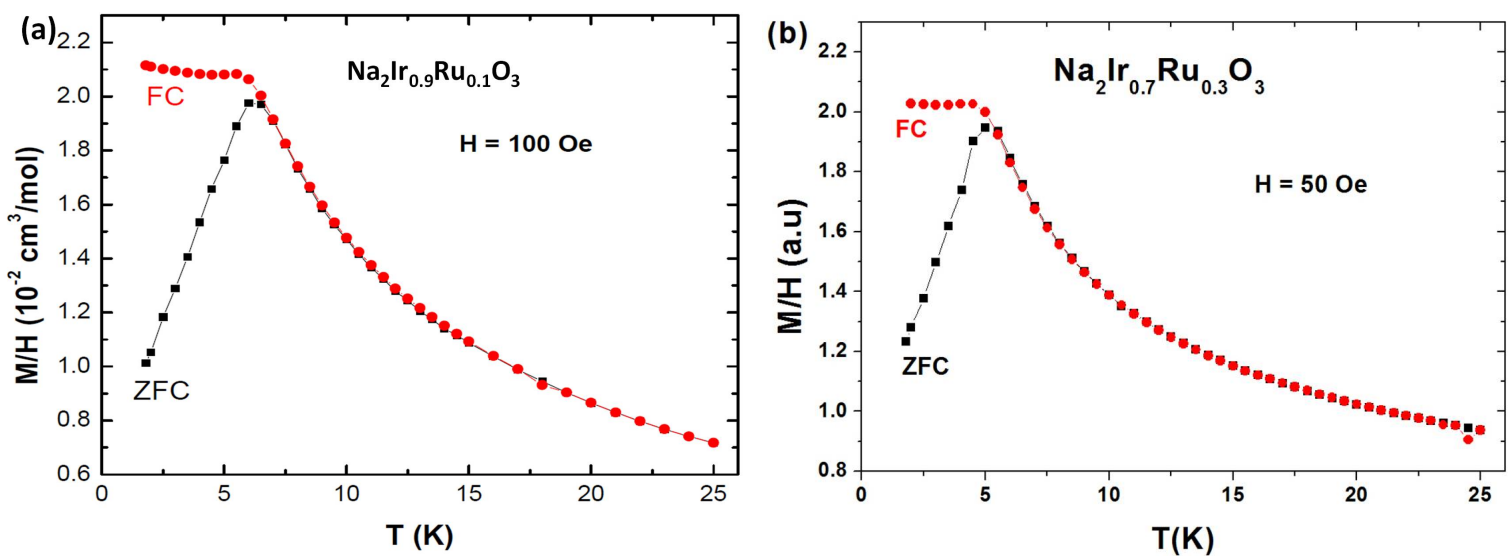

Figure 5.11: FC and ZFC susceptibility of the (a) $10 \% \mathrm{Ru}$ doped (b) $30 \% \mathrm{Ru}$ doped $\mathrm{Na}_{2} \mathrm{IrO}_{3}$ crystals measured at low field. Data measured by Yogesh Singh.

Isolelectronic doping at $\mathrm{A}$-site of $\mathrm{A}_{2} \mathrm{IrO}_{3}$ has shown that both $\mathrm{Na}$ - and $\mathrm{Li}$ - system have very different magnetic property. A continuous evolution of AF magnetic ordering is observed in the $\left(\mathrm{Na}_{1-x} \mathrm{Li}_{x}\right)_{2} \mathrm{IrO}_{3}$ phase diagram. To understand the Ir-magnetism one 


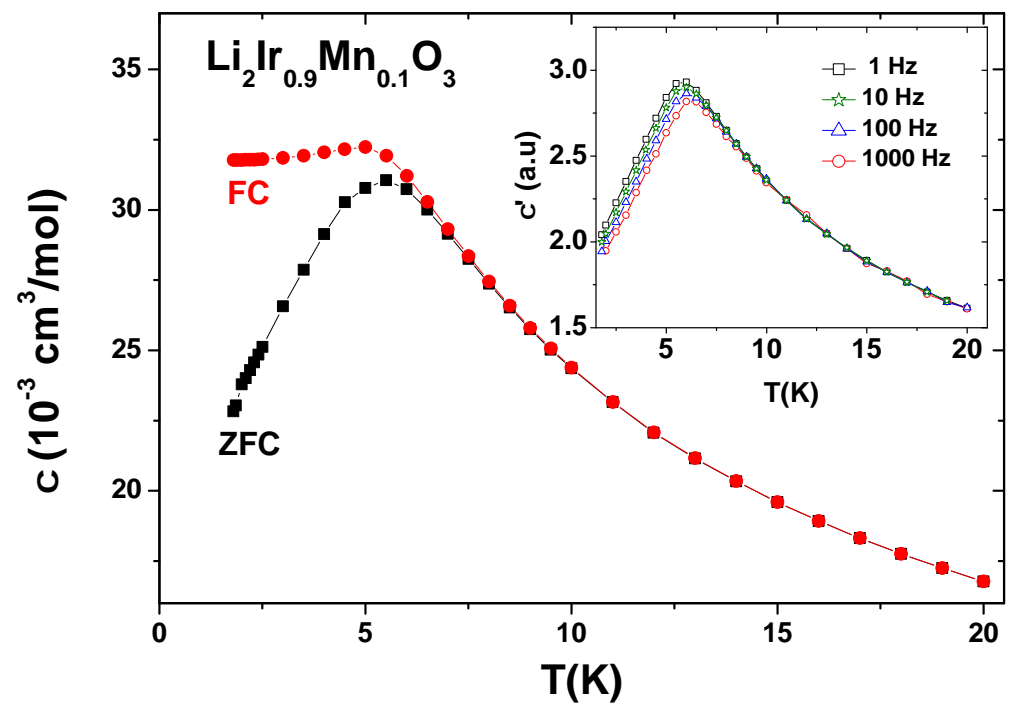

Figure 5.12:
FC and ZFC
susceptibility of
the $10 \%$ Mn doped
$\mathrm{Li}_{2} \mathrm{IrO}_{3}$ polycrys-
tals measured at
50 Oe, (inset) fre-
quency dependent
ac susceptibil-
ity $\left(\chi^{\prime}\right)$ versus
temperature mea-
sured at different
frequency.

has to substitute magnetic site in $\mathrm{A}_{2} \mathrm{IrO}_{3}$. So, magnetic impurity was doped at Ir-site. Ir $S=1 / 2$ site was substituted by $S=1$ and $S=3 / 2$ ion.

$\mathrm{Na}_{2} \mathrm{Ir}_{1-x} \mathrm{Ru}_{x} \mathrm{O}_{3}$ crystals were grown by the same method like $\mathrm{Na}_{2} \mathrm{IrO}_{3}$. [78 $]^{3} \mathrm{Ru}^{4+}$ has $4 d$-electrons so in low-spin state it has effective $S=1$. In the Fig. 5.11 (a) and (b) magnetic measurement on $x=0.1$ and $x=0.3, \mathrm{Na}_{2} \mathrm{Ir}_{1-x} \mathrm{Ru}_{x} \mathrm{O}_{3}$ crystals are shown respectively. A strong hysteresis is observed in field cooled (FC) and zero field cooled (ZFC) susceptibility which is characteristic feature for spin-glass (SG) freezing. The cusp in the ZFC susceptibility gives $\mathrm{SG}$ freezing temperature $T_{g}=5 \mathrm{~K}$. For both $x=0.1$ and $x=0.3$ $T_{g}$ is same, the cusp occurs at same temperature. For a frustrated system doping with magnetic impurity should give spin-glass freezing but the invariance of $T_{g}$ with increasing magnetic impurity is not much informative for understanding strength of magnetic exchange interactions.

Magnetic impurity doping was done on $\mathrm{Li}_{2} \mathrm{IrO}_{3}$ polycrystals also. $\mathrm{Li}_{2} \mathrm{RuO}_{3}$ has very different property: $\mathrm{Ru}-\mathrm{Ru}$ dimers form in $\mathrm{Li}_{2} \mathrm{RuO}_{3}$ at $600 \mathrm{~K}$, and make effective $S=$ 0.[79] Even very recent study on $\mathrm{Li}_{2} \mathrm{Ir}_{1-x} \mathrm{Ru}_{x} \mathrm{O}_{3}$ doping series reported $\mathrm{Ru}$-Ru dimerization above $x>0.2$.[80] Hence physics of this system is totally different but interesting. $\mathrm{Mn}^{4+}$ was doped at Ir-site in $\mathrm{Li}_{2} \mathrm{IrO}_{3} . \mathrm{Mn}^{4+}$ has $3 d$-electrons hence $S=3 / 2$ is realized by Hund's coupling. $\mathrm{Li}_{2} \mathrm{Ir}_{1-x} \mathrm{Mn}_{x} \mathrm{O}_{3}$ polycrystals were prepared by solid state reaction. XRD confirmed single phase. There were no pure $\mathrm{Li}_{2} \mathrm{MnO}_{3}$ phase as impurity in any doped batch. Otherwise magnetic measurement would detect long range AF ordering at $40 \mathrm{~K}$ for $\mathrm{Li}_{2} \mathrm{MnO}_{3}$ [53]. Increase in Curie constant for each Mn-doped batch confirmed an increase in

\footnotetext{
${ }^{3} \mathrm{Na}_{2} \mathrm{Ir}_{1-x} \mathrm{Ru}_{x} \mathrm{O}_{3}$ crystals were grown and studied by Yogesh Singh when he was a postdoc in our lab.
} 
effective fluctuating moment which in turn confirms $S=3 / 2$ magnetic impurity doping at the Ir $S=1 / 2$ site. In Fig. 5.12 low temperature static and dynamic magnetic susceptibility measurements are presented for $x=0.1$. A clear hysteresis is observed between the FC and ZFC susceptibility at low field $(5 \mathrm{mT})$ which confirms SG freezing. Such SG freezing is observed for some other values of $x$ also. The position of the cusp in ZFC susceptibility and ac susceptibility $\left(\chi^{\prime}\right)$ at $1 \mathrm{~Hz}$ determines SG temperature $T_{g}=6 \mathrm{~K}$ (inset). Strong frequency dependence of the ac susceptibility further confirms SG freezing.

Hence all the magnetic impurity doping in $\mathrm{Na}_{2} \mathrm{IrO}_{3}$ and $\mathrm{Li}_{2} \mathrm{IrO}_{3}$ show $\mathrm{SG}$ freezing at the same temperature around $6 \mathrm{~K}$. A relative strength of the magnetic exchange interaction would be understood from change in the spin dynamics but an unchanged freezing temperature puts bar on it.

\subsection{Nonmagnetic dilution of Ir-magnetism: $\mathrm{A}_{2}\left(\operatorname{Ir}_{1-x} \mathrm{Ti}_{x}\right) \mathrm{O}_{3}$}

${ }^{4}$ INS measurement by Choi et.al. showed that $\mathrm{Na}_{2} \mathrm{IrO}_{3}$ and $\mathrm{Li}_{2} \mathrm{IrO}_{3}$ has totally different magnetic structure $[36,73]$. $\left(\mathrm{Na}_{1-x} \mathrm{Li}_{x}\right)_{2} \mathrm{IrO}_{3}$ phase diagram indicated very different magnetic origin for $\mathrm{Na}$ - and Li-system despite same long range $\mathrm{AF}$ ordering temperature. This may happen due to the different relative importance of exchange interactions between Irspins within the Ir-honeycomb lattice. So far following magnetic exchange interactions are discussed in honeycomb lattice iridates:

- Nearest neighbor Heisenberg $(J)$ and Kitaev $(K)$ interactions.

- Second nearest neighbor Heisenberg $\left(J_{2}\right)$ and Kitaev $\left(K_{2}\right)$ interactions.

- Third nearest neighbor Heisenberg $\left(J_{3}\right)$ interaction.

Different models have been used to describe magnon spectra and bulk property for both of the systems. Very confusing and sometimes contradictory results appeared. To identify the proper model and to write a proper spin-Hamiltonian one has to identify the most important magnetic exchange interactions, whether it is the nearest neighbor interactions or beyond next neighbor interactions. One way to answer this question is to perturb the

\footnotetext{
${ }^{4}$ In this doping study, crystal growth, EDX, powder XRD, thermodynamic and magnetic measurements were done by myself. Thermodynamic measurements below $0.5 \mathrm{~K}$ were done by Yoshi Tokiwa utilizing a dilution refrigerator. The detailed investigation of the $\mathrm{A}_{2}\left(\operatorname{Ir}_{1-x} \mathrm{Ti}_{x}\right) \mathrm{O}_{3}$ phase diagram is published in Ref. [81]. The experimental part of this section on $\mathrm{Na}_{2}\left(\operatorname{Ir}_{1-x} \mathrm{Ti}_{x}\right) \mathrm{O}_{3}$ and $\mathrm{Li}_{2}\left(\operatorname{Ir}_{1-x} \mathrm{Ti}_{x}\right) \mathrm{O}_{3}$ investigations is mainly copied from Ref. [81] which has been written by myself and corrected by my co-authors.
} 


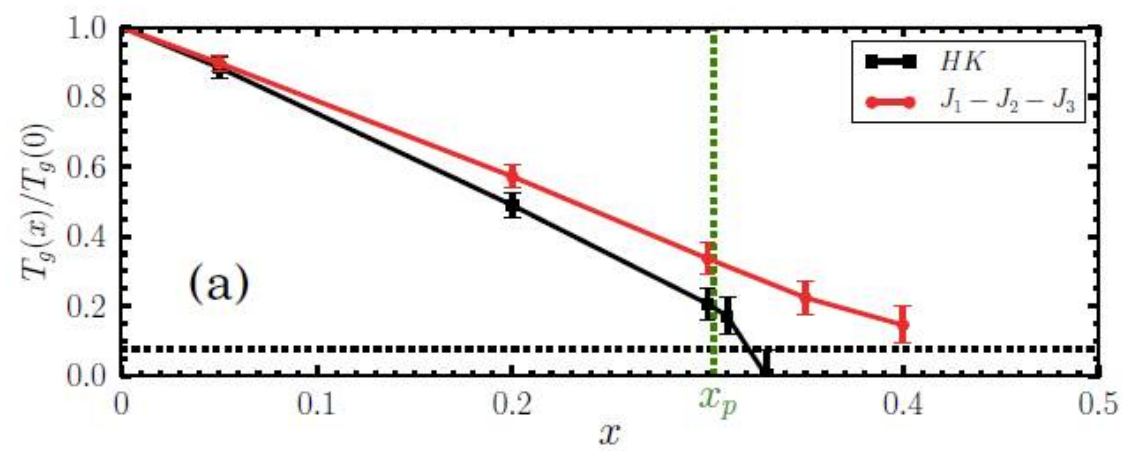

Figure 5.13: Ordering/freezing temperature. (a) $\mathrm{T}_{g}(\mathrm{x})=\mathrm{T}_{g}(\mathrm{x}=0)$ as function of doping level $\mathrm{x}$ for the $\mathrm{KH}$ and $J_{1}-J_{2}-J_{3}$ models for $J \perp / J=0.01$. The vertical dashed line locates the $2 \mathrm{D}$ percolation threshold $x_{p}$; the horizontal dashed line marks temperatures below which we are unable to reach equilibrium in our MC simulations for the HK model. Reprinted from [83].

Ir-magnetism and observe the change in the honeycomb-lattice. Magnetic impurity doping at Ir-site gives a robust change, spin-glass (SG) freezing at same temperature $T_{\mathrm{g}} \approx 6 \mathrm{~K}$ and the SG behavior does not depend on the amount of magnetic impurity.

Other possibility is systematic dilution of $S_{\text {eff }}=1 / 2$ fluctuating moments by substitution of non-magnetic dopants at the Ir site. It should be an effective tool because removing the spins dilute the interaction strengths between the spins. A system having stronger nearest neighbor exchange would behave much differently than the system with substantial further neighbor exchange upon dilution of magnetic exchange. The Ir-atom in $\mathrm{A}_{2} \mathrm{IrO}_{3}$ can be substituted by $\mathrm{Ti}$ ion which will result: $\mathrm{A}_{2}\left(\mathrm{Ir}_{1-x} \mathrm{Ti}_{x}\right) \mathrm{O}_{3}$. $\mathrm{Ti}^{4+}$ has no d-electrons, effective spin $\operatorname{zero}(S=0)$. The reason to chose Ti as substituent for Ir is that $\mathrm{Ti}^{4+}$ and $\operatorname{Ir}^{4+}$ have a very similar ionic radius. In compounds where Ir and Ti occupy different sites this causes a severe problem due to site exchange, [82] while in our case it assures a good statistical mixing of $\mathrm{Ir}$ and $\mathrm{Ti}$ in the diluted systems. Hence single crystals of $\mathrm{Na}_{2}\left(\mathrm{Ir}_{1-x} \mathrm{Ti}_{x}\right) \mathrm{O}_{3}$ and well ordered poycrystals of $\operatorname{Li}_{2}\left(\operatorname{Ir}_{1-x} \mathrm{Ti}_{x}\right) \mathrm{O}_{3}$ were synthesized. At the same time a paper came out from Eric C. Andrade et. al about different behavior of nearest neighbor HK model and $J_{1}-J_{2}-J_{3}$ model in honeycomb lattice iridates upon depletion of Ir-spins using Monte Carlo (MC) calculation. [83]

\subsubsection{Theoretical Prediction}

The MC calculation compared the HK model (2.8) and $J_{1}-J_{2}-J_{3}$ model (2.12) in case of $\mathrm{A}_{2} \mathrm{IrO}_{3}$ under magnetic depletion. They showed in their paper zig-zag ordering of $\mathrm{A}_{2} \mathrm{IrO}_{3}$ enters into a spin glass disordered sate by magnetic dilution. [83]. As both of this models 
describes a frustrated spin interactions it is an obvious destiny. But they showed that spin glass temperature $T_{\mathrm{g}}$ varied differently with degree of dilution (doping level, $x$ ) for both the models (Fig. 5.13).Final phase diagram was drawn in a weak inter-layer coupling $\operatorname{limit}(J \perp / J=0.01)$ which seemed so far justified in case of $\mathrm{A}_{2} \mathrm{IrO}_{3}$. In case of nearest neighbor HK model normalized $T_{\mathrm{g}}(x)$ drops linearly and very sharply with $x$ and goes to zero before the percolation limit of the $2 \mathrm{D}$ honeycomb lattice $\left(x_{p}=0.3\right)$. But in case of long range $J_{1}-J_{2}-J_{3}$ model, normalized $T_{\mathrm{g}}(x)$ has finite value even beyond $x_{p}$ and deviates from linear behavior above $x_{p}$. Hence comparing our results of $\mathrm{A}_{2}\left(\operatorname{Ir}_{1-x} \mathrm{Ti}_{x}\right) \mathrm{O}_{3}$ with this phase diagram from MC calculation one can draw conclusion about relative importance of different exchange interactions.

\subsubsection{Experimental details}

$\mathrm{Na}_{2}\left(\mathrm{Ir}_{1-x} \mathrm{Ti}_{x}\right) \mathrm{O}_{3}$ single crystals were grown using a similar method as for $\mathrm{Na}_{2} \mathrm{IrO}_{3}$, by prereacting $\mathrm{Na}_{2} \mathrm{CO}_{3}$, Ir metal powder and $\mathrm{TiO}_{2}$ powder at $750^{\circ} \mathrm{C}$ upto $900^{\circ} \mathrm{C}$. The subsequent crystal growth was done with $10 \%$ extra $\mathrm{IrO}_{2}$ in between $1030-1050^{\circ} \mathrm{C}$. Unfortunately, this method only worked for compositions $x \leq 0.3$. At larger $x$ only a solid melt of $\mathrm{Na}_{2} \mathrm{TiO}_{3}$ was obtained and no $\mathrm{Na}_{2}\left(\mathrm{Ir}_{1-x} \mathrm{Ti}_{x}\right) \mathrm{O}_{3}$ crystals formed. $\mathrm{Na}_{2} \mathrm{TiO}_{3}$ has a very low melting point of $180^{\circ} \mathrm{C}$ which causes this problem for $x>0.3$. Since chemistry and crystal structure of $\mathrm{Na}_{2} \mathrm{TiO}_{3}$ differs from $\mathrm{Na}_{2} \mathrm{IrO}_{3}$, attempts to synthesize single-phase $\mathrm{Na}_{2}\left(\mathrm{Ir}_{1-x} \mathrm{Ti}_{x}\right) \mathrm{O}_{3}$ polycrystals for $x>0.3$ have failed.

For $\mathrm{Li}_{2}\left(\mathrm{Ir}_{1-x} \mathrm{Ti}_{x}\right) \mathrm{O}_{3}$ well ordered single phase polycrystals was prepared up to $x=0.55$ by solid state reaction. At higher doping $\operatorname{Li}_{2}\left(\operatorname{Ir}_{1-x} \mathrm{Ti}_{x}\right) \mathrm{O}_{3}$ polycrystals become disordered probably due to a site exchange between $\mathrm{Li}$ and $\mathrm{Ti}$. For polycrystal synthesis $\mathrm{Li}_{2} \mathrm{CO}_{3}$, Ir metal powder and $\mathrm{TiO}_{2}$ were mixed and reacted in the open furnace at $700-1000^{\circ} \mathrm{C}$ in $100^{\circ} \mathrm{C}$ steps after repetitive grinding and pelletizing in each step.

Phase purity and structural ordering were verified from powder x-ray diffraction (XRD). For the elemental quantification of the Ir and Ti content several spots on various pieces of each batch had been studied by the energy dispersive x-ray (EDX) method. Here $x$ always denotes the actual Ti concentration. Magnetization, ac susceptibility and specific heat measurements were conducted in the Quantum Design MPMS and PPMS. Thermodynamic measurements below $0.4 \mathrm{~K}$ were performed in a dilution refrigerator in our lab by Yoshi Tokiwa on selected $\mathrm{Li}_{2}\left(\mathrm{Ir}_{1-x} \mathrm{Ti}_{x}\right) \mathrm{O}_{3}$ samples. [84] 


\subsubsection{Results and Discussions}

\subsubsection{1 $\quad \mathrm{Na}_{2}\left(\operatorname{Ir}_{1-x} \mathbf{T i}_{x}\right) \mathbf{O}_{3}$ Results}

Elemental analysis: Ir:Ti ratio was determined on each batch of $\mathrm{Na}_{2}\left(\operatorname{Ir}_{1-x} \mathrm{Ti}_{x}\right) \mathrm{O}_{3}$ single

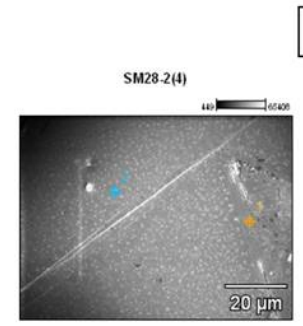

$$
X=0.015
$$
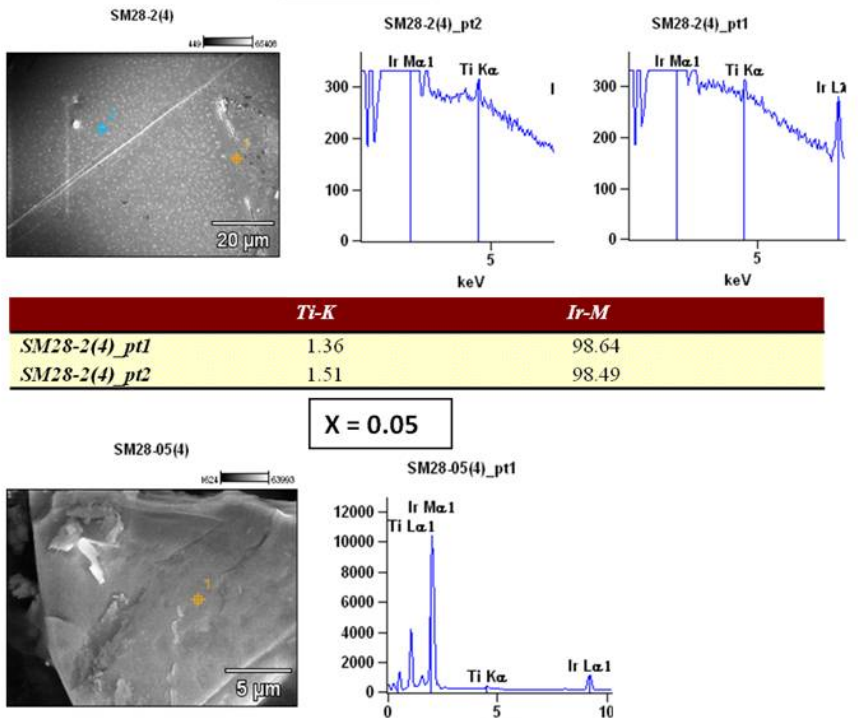

$1.36 \quad 98.64$

98.49

$x=0.05$
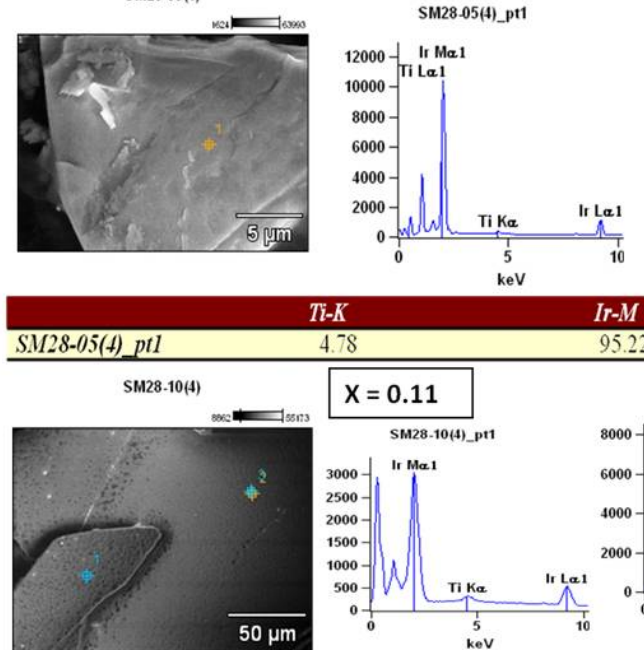

\section{Ir-M}
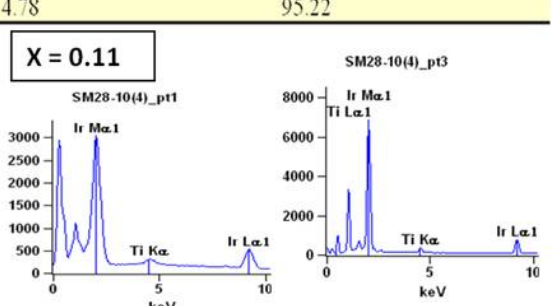

$\begin{array}{lc}\text { Figure } & \mathbf{5 . 1 4 :} \\ \text { Elemental } & \text { anal- } \\ \text { ysis of } x \quad= \\ 0.015,0.05,0.11 \\ \mathrm{Na}_{2}\left(\mathrm{Ir}_{1-x} \mathrm{Ti}_{x}\right) \mathrm{O}_{3} \text { crys- } \\ \text { tals by } \text { Energy } \\ \text { dispersive } & \text { x-ray } \\ (\text { EDX }) . & \end{array}$

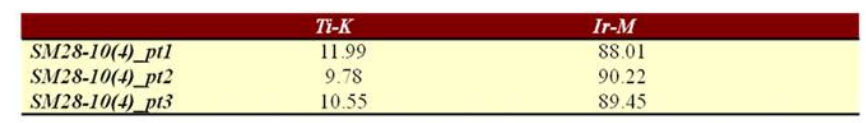

crystals by EDX. In Fig. 5.14 EDX measurement for $x=0.015,0.05$ and 0.11 is shown. These values of $x$ were determined from the average value of the measurements on several points (pt1, pt2 etc. in the respective tables in Fig. 5.14) of a single piece of crystal. Such measurement was done at least on 2-3 single crystals of same batch to ensure homogeneity in a growth. With increasing doping, height of Ti $K_{\alpha}$ peak is increasing. For $x=0.015$, counts for Ti $K_{\alpha}$ is very small compared to Ir $M_{\alpha}$ line so in Fig. 5.14 that spectra is shown zoomed around $\mathrm{Ti} K_{\alpha}$ peak. In the respective tables of the Fig. 5.14 Ir and Ti percentages are shown for the three doping. It is clear from these table that values does not vary much over one piece of crystal which confirms a homogeneous substitution. 


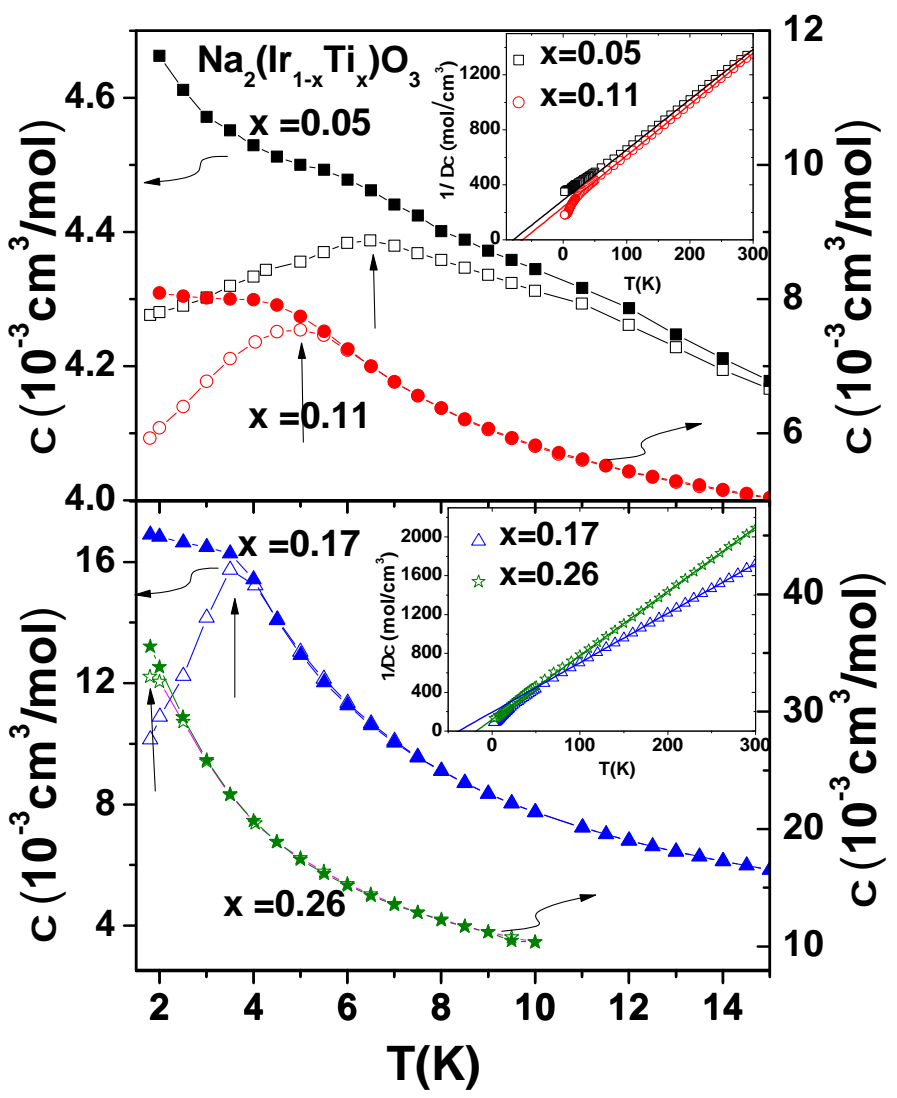

Figure 5.15: Field cooled (FC) and zero field cooled (ZFC) susceptibility vs. temperature as indicated by filled and open symbols, respectively, for $\mathrm{Na}_{2}\left(\mathrm{Ir}_{1-x} \mathrm{Ti}_{x}\right) \mathrm{O}_{3} \quad$ with $x=0.05,0.11$ (upper panel) and $0.17,0.26$ (lower panel). Vertical arrows mark $\mathrm{T}_{g}$. Respective insets display $1 / \Delta \chi\left(\right.$ with $\left.\Delta \chi=\chi-\chi_{0}\right)$ vs. T. Solid lines indicate Curie-Weiss behavior. Reprinted from [81].

Magnetism: Magnetization measurements on $\mathrm{Na}_{2}\left(\mathrm{Ir}_{1-x} \mathrm{Ti}_{x}\right) \mathrm{O}_{3}$ single crystals show that for all investigated $x$ the magnetic susceptibility $\chi=M / H$ follows Curie-Weiss (CW) behavior (Eqn. 4.7), see insets of Fig. 5.15. This implies that with increasing degree of dilution by Ti substitution the local moment behavior persists in Na-system. In the CW fit the Curie constant $(C)$ decreases from 0.41 at $x=0$ to 0.15 at $\mathrm{x}=0.26$ signifies decrease in the effective fluctuating moment $\mu_{e f f}$. This confirms with the dilution of Ir moments by nonmagnetic Ti. Small temperature independent van Vleck contributions $\left(\chi_{0}\right)$ are of order $10^{-5} \mathrm{~cm}^{3} / \mathrm{mol}$. Most significantly the AF Weiss temperature $\left(\Theta_{W}\right)$ changes from $-125 \mathrm{~K}$ at $x=0$ to $-18 \mathrm{~K}$ for $x=0.26$ indicating a continuous decrease of the CW scale with magnetic depletion for the Na-system. Low temperature (below $10 \mathrm{~K}$ )) small cusps were observed for all the $\chi(T)$ measurement. Usually such low- $T$ feature in susceptibility signifies some magnetically ordered ( short range or long range) ground state.

To check any possibility of short range magnetic order like spin-glass (SG) freezing thermal hysteresis of magnetic susceptibility was measured. For $x=0.05,0.11,0.17$ and 0.26 $\left(\mathrm{Na}_{1-x} \mathrm{Li}_{x}\right)_{2} \mathrm{IrO}_{3}$ crystals field-cooled (FC) and zero-field cooled (ZFC) measurements at very low field of $5 \mathrm{mT}$ shown in Fig. 5.15 display cusps for ZFC and a clear separation 


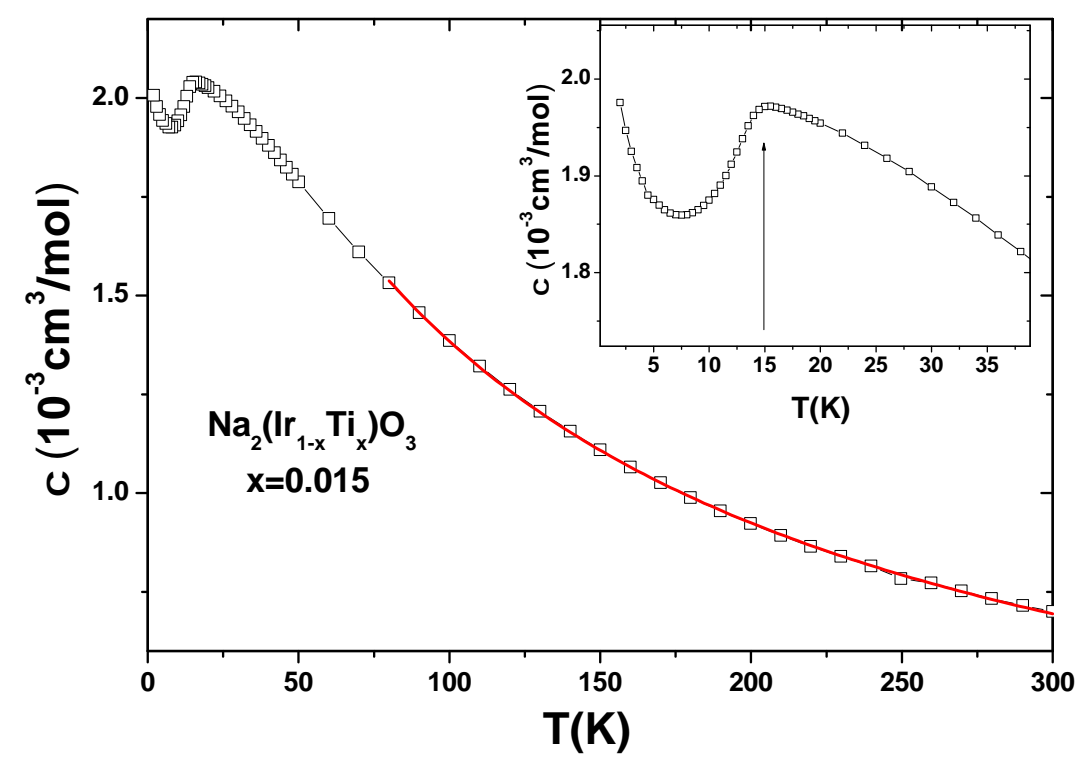

Figure 5.16: Magnetic susceptibility $(\chi)$ vs. $T$ for $\mathrm{Na}_{2}\left(\operatorname{Ir}_{1-x} \mathrm{Ti}_{x}\right) \mathrm{O}_{3}$ single crystal $x=$ 0.015. The red line indicates Curie-Weiss behavior. (inset) Zoomed view on low temperature peak, vertical arrow marks $T_{N}=15 \mathrm{~K}$. Reprinted from [81].

between FC and ZFC traces at low $T$, which are characteristic signatures for spin-glass (SG) behavior. The freezing temperature $T_{\mathrm{g}}$ is determined from the kinks indicated by vertical arrows in Fig. 5.15. For these doping $T_{\mathrm{g}}$ reduces from $6.8 \mathrm{~K}$ for $x=0.05$ to $2 \mathrm{~K}$ for $x=0.26$. For the lowest Ti-substituted $\mathrm{Na}_{2}\left(\operatorname{Ir}_{1-x} \mathrm{Ti}_{x}\right) \mathrm{O}_{3}$ single crystal with $x=0.015$ long range magnetic ordering is still present below $15 \mathrm{~K}$. In Fig. $5.16 \chi$ versus $T$ is shown for that sample. An anomaly with similar shape as for undoped $\mathrm{Na}_{2} \mathrm{IrO}_{3}$ [52] is visible at $15 \mathrm{~K}$. Any hysteresis in field cooled and zero-field cooled data was not observed. So the anamoly in susceptibility signifies long-range AF ordering below $15 \mathrm{~K}$ for $x=0.015$. The red straight line in the figure represents the $\mathrm{CW}$ behavior with $\theta_{W}=-101 \mathrm{~K}$.

This spin spin-glass feature was further confirmed from frequency dependent ac susceptibility measurement. In Fig.5.17 frequency dependent ac $\operatorname{susceptibility}\left(\chi^{\prime}\right)$ for $\mathrm{x}=0.17$ $\mathrm{Na}_{2}\left(\mathrm{Ir}_{1-x} \mathrm{Ti}_{x}\right) \mathrm{O}_{3}$ is plotted. It shows a frequency dependent sharp cusp at $\mathrm{T}_{g}$. At $1 \mathrm{~Hz}$ the cusp is very sharp and having peak at $T_{\mathrm{g}}$, with the increasing frequency this cusp shifted towards higher temperature and also little broadened with increasing frequency. To measure this frequency dependence of spin glass temperature $\left(T_{f}\right)$ at different frequency $(\omega)$ is plotted in logarithmic scale in the inset of Fig. 5.17. Frequency dependence is measured by $\Delta T_{f} /\left(T_{\mathrm{g}} \Delta \log \omega\right)$ which measures change of $T_{\mathrm{g}}$ in per decade of frequency divided by $\mathrm{T}_{g}$. For canonical spin-glasses it is around 0.02. But as $\operatorname{Li}_{2}\left(\operatorname{Ir}_{1-x} \mathrm{Ti}_{x}\right) \mathrm{O}_{3}$ is a insulating local moment system, change in $\mathrm{T} g$ is one order of magnitude higher having $\Delta T_{f} /\left(T_{\mathrm{g}} \Delta \log \omega\right)=0.11$. 


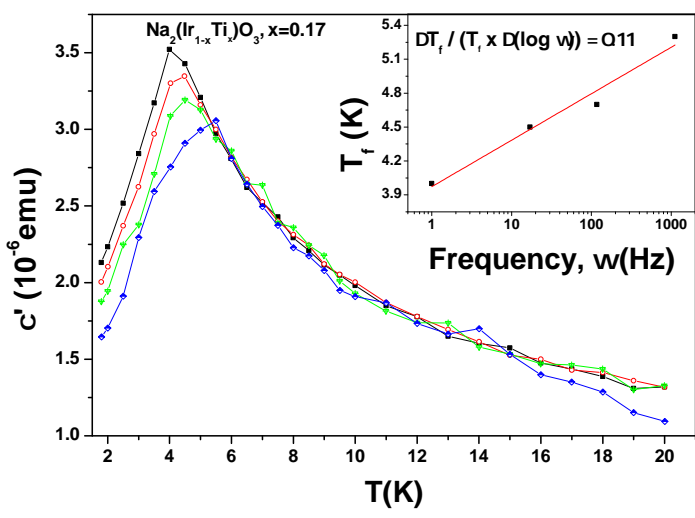

Figure 5.17: Frequency $(\omega)$ dependence of ac susceptibility $\left(\chi^{\prime}\right)$ versus $\mathrm{T}$ measurement around $\mathrm{T}_{g}$. Shows a clear cusp and shift of the cusp with frequency. Inset shows position of the maxima $\mathrm{T}_{f}$ at different $\omega$ in logarithmic scale, it follows a linear behavior and the slope giving frequency shift parameter per decade. Reprinted from [81].

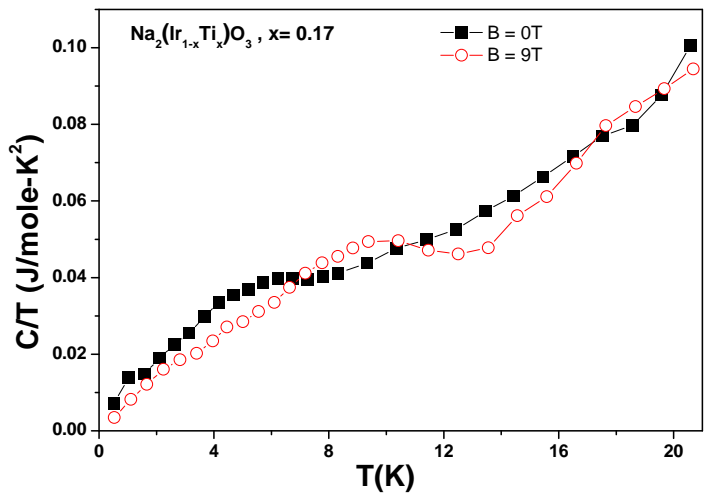

Figure 5.18: Heat capacity divided by temperature $(\mathrm{C} / \mathrm{T})$ measured at $\mathrm{B}=0 \mathrm{~T}$ and $9 \mathrm{~T}$ for $\mathrm{x}=0.17 \mathrm{Na}_{2}\left(\operatorname{Ir}_{1-x} \mathrm{Ti}_{x}\right) \mathrm{O}_{3}$. It shows a broad hump around $1.4 \mathrm{~T}_{g}$ which almost disappears upon applying field. Reprinted from [81].

Heat capacity: To confirm that for $x \geq 0.05$ there is no long range magnetic order the heat capacity has been measured. Crystals were very tiny and hence it was pretty challenging to obtain good data. In fig. 5.18 the heat capacity divided by temperature $(C / T)$ is plotted for $\mathrm{Na}_{2}\left(\operatorname{Ir}_{1-x} \mathrm{Ti}_{x}\right) \mathrm{O}_{3}, x=0.17$ at $B=0$ and $9 \mathrm{~T}$. It shows a broad hump around $6 \mathrm{~K}$ which is 1.4 times its $T_{\mathrm{g}}$ determined from ac-susceptibility. Upon applying $9 \mathrm{~T}$ magnetic field it broadens and shifts to higher temperatures. Such behavior is characteristic for spin-glass freezing and it confirms the absence of long-range order.

Hence all results confirms $\mathrm{Na}_{2}\left(\operatorname{Ir}_{1-x} \mathrm{Ti}_{x}\right) \mathrm{O}_{3}$ shows spin-glassy behavior with continuous decrease of $\mathrm{T}_{g}$ with increasing level of dilution (x).

\subsubsection{2 $\quad \mathbf{L i}_{2}\left(\operatorname{Ir}_{1-x} \mathrm{Ti}_{x}\right) \mathrm{O}_{3}$ Results}

Elemental Anlysis: $\mathrm{Li}$ is insensitive to EDX as it is lightest metal element, so 2:1:3 composition of $\mathrm{Li}$, Ir and $\mathrm{O}$ only can be verified from rietveld analysis of powder XRD. Ir to Ti ratio was determined from EDX. In Fig. 5.19 EDX spectra for all $\operatorname{Li}_{2}\left(\operatorname{Ir}_{1-x} \mathrm{Ti}_{x}\right) \mathrm{O}_{3}$ samples are shown. In all the spectra the black arrow points towards Ti peak. For SM29-10(10\% Ti doped $\mathrm{Li}_{2} \mathrm{IrO}_{3}$ )this $\mathrm{Ti} K_{\alpha}$ peak is smallest and with the increasing $x$ relative height of that peak increases in comparison to the Ir $M_{\alpha}$. From these spectra $x$ was determined for each batch of doping. This determination was done on several points of same piece and on several 


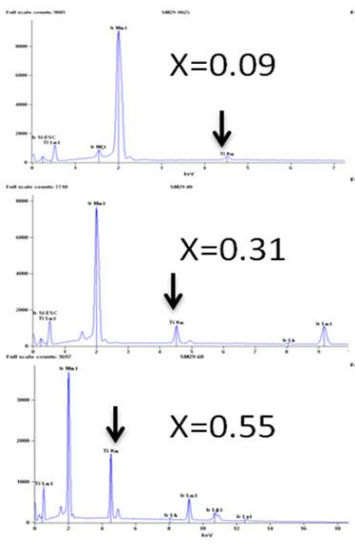

Figure 5.19: Energy dispersive x-ray (EDX) spectra measured on $\mathrm{Li}_{2}\left(\mathrm{Ir}_{1-x} \mathrm{Ti}_{x}\right) \mathrm{O}_{3}$ polycrystalline pellets. The downward arrows point Ti-K $\alpha$ peak. Other big one is Ir-M $\alpha$ peak.

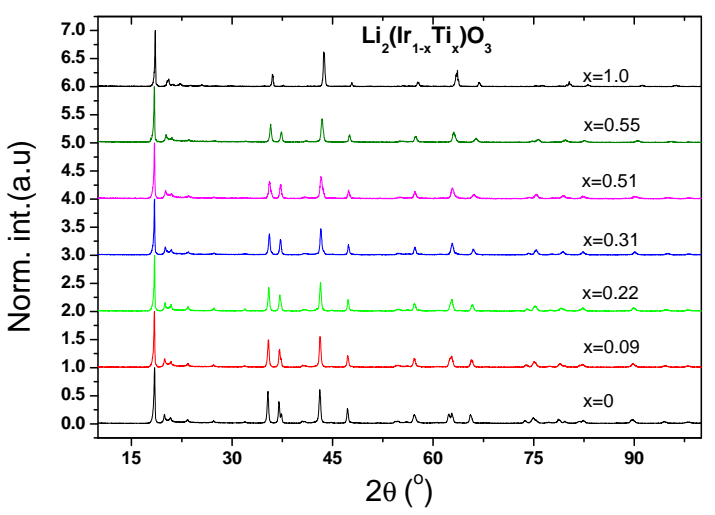

Figure 5.20: Powder x-ray diffraction (XRD) spectra of $\mathrm{Li}_{2}\left(\mathrm{Ir}_{1-x} \mathrm{Ti}_{x}\right) \mathrm{O}_{3}$ polycrystal measured between $2 \theta=10^{\circ}$ to $100^{\circ}$. Theses spectra are used to calculate respective lattice parameters for all the dpoping. Reprinted from [81].

pieces of same batch, average value of all such measurements was confirmed as actual $x$ for each batch of doping. In the Fig. 5.19 all determined $x$ values are pointed out.

Structural Characterization: After elemental analysis next part was to confirm the structure and phase purity. Powder XRD measurement of $\mathrm{Li}_{2}\left(\operatorname{Ir}_{1-x} \mathrm{Ti}_{x}\right) \mathrm{O}_{3}$ for each $x$ was fitted with $\mathrm{C} 2 / \mathrm{m}$ crystal structure by Rietveld method. For $\mathrm{x} \leq 0.55 \mathrm{Li}_{2}\left(\mathrm{Ir}_{1-x} \mathrm{Ti}_{x}\right) \mathrm{O}_{3}$ structurally well ordered samples were obained (XRD peaks between $2 \theta 19$ to $33^{\circ}$ are well separate in Fig. 5.20). Also all the XRD peaks for $\mathrm{x} \leq 0.55$ matching with $\mathrm{Li}_{2} \mathrm{IrO}_{3}(x=0)$ XRD peak. But for $\mathrm{x}=1$ i.e. for $\mathrm{Li}_{2} \mathrm{TiO}_{3} \mathrm{XRD}$ peaks could not fitted with $\mathrm{C} 2 / \mathrm{m}$ crystal structure. $\mathrm{Li}_{2} \mathrm{TiO}_{3}$ has $\mathrm{C} 2 / \mathrm{c}$ crystal structure. One of the characteristic seen feature in Fig. 5.20 : XRD peak at $2 \theta=37^{\circ}$ is very small compared to same in $x<1$ which is (004) peak in $\mathrm{C} 2 / \mathrm{c}$ crystal structure. $\mathrm{Li}_{2} \mathrm{TiO}_{3}$ lattice parameters are transformed into $\mathrm{C} 2 / \mathrm{m}$ by this transformation equations : $\mathbf{a}^{\prime}=-\mathbf{a}, \mathbf{b}^{\prime}=-\mathbf{b}, \mathbf{c}^{\prime}=\mathbf{a}+2 \mathbf{c}, \mathrm{c}^{\prime}=\sqrt{ }\left(\mathrm{a}^{2}+4 \mathrm{c}^{2}+4 \mathrm{ac} \cos \beta\right), \sin \beta^{\prime}=\frac{2 c}{c^{\prime}}$ $\left(\operatorname{dashed}\left({ }^{\prime}\right)\right.$ are $\mathrm{C} 2 / \mathrm{c}$ lattice parameter and others are $\left.\mathrm{C} 2 / \mathrm{m}\right)$.

Lattice parameters of $\mathrm{Li}_{2}\left(\mathrm{Ir}_{1-x} \mathrm{Ti}_{x}\right) \mathrm{O}_{3}$ are plotted in Fig. 5.21. They were calculated by structural refinement of XRD spectra using Rietveld method. In the structural refinement it was assumed that Ti goes only in the Ir site. Refinement converged and good enough fitting parameters $\left(\mathrm{R}_{p}\right.$ and $\left.\mathrm{R}_{w p}\right)$ were obtained only when a little site exchange $(f<0)$ was introduced between Ir site $(4 g)$ and Li site $(2 a)$ in the center honeycomb. Hence occupancy at the iridium site was assumed $(1-f-x) \mathrm{Ir}+x \mathrm{Ti}+f \mathrm{Li}$ and at the honeycomb center site $(1-2 f) \mathrm{Li}+2 f \mathrm{Ir}$ to balance the stoichiometry. Lattice parameters of $\mathrm{Li}_{2} \mathrm{IrO}_{3}$ and $\mathrm{Li}_{2} \mathrm{TiO}_{3}$ vary only from 0.5 to $1 \%$. The $b$ lattice constant changes almost linearly with $x$ from 0 to 


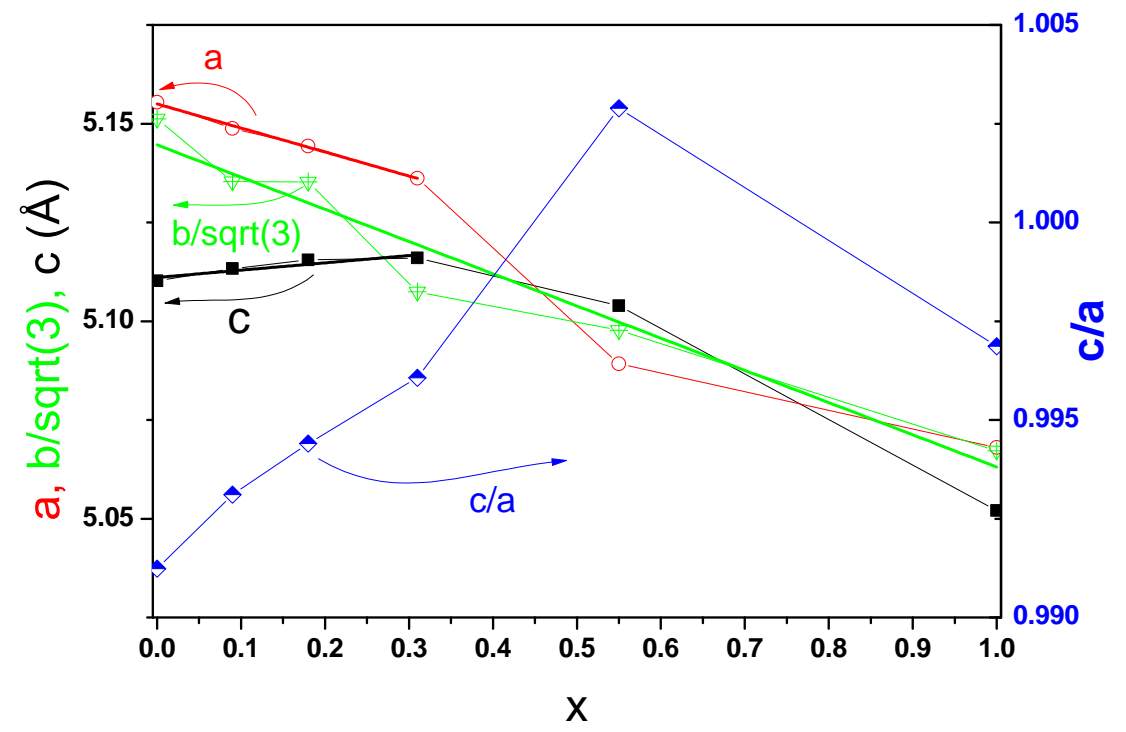

Figure 5.21: Lattice parameters of $\mathrm{Li}_{2}\left(\mathrm{Ir}_{1-x} \mathrm{Ti}_{x}\right) \mathrm{O}_{3}$ obtained from Rietveld analysis of the powder XRD data. Reprinted from [81].

1 and $a$ and $c$ vary non-linearly. It is confirmed that by Ti substitution there is no change in crystal structure only a nominal change in lattice parameters.

Magnetism: Magnetic molar suscptibility $(\chi(T))$ was measured on polycrystals of $\mathrm{Li}_{2}\left(\operatorname{Ir}_{1-x} \mathrm{Ti}_{x}\right) \mathrm{O}_{3}$ which follows $\mathrm{CW}$ behavior. In Fig. 5.22 inset CW fitting of $1 / \Delta \chi$ versus $T$ between 100 and $300 \mathrm{~K}$ is plotted. $\Delta \chi=\chi-\chi_{\circ}, \chi_{\circ}$ is obtained from $\mathrm{CW}$ fitting of $\chi(T)$ vs. $T$ data at high temperature which ranges between $-1 \cdot 10^{-5} \mathrm{~cm}^{3} / \mathrm{mol}$ and $-5 \cdot 10^{-5}$ $\mathrm{cm}^{3} / \mathrm{mol}$. From the CW fitting in the inset Fig. 5.22 Curie constant is obtained which gives effective fluctuating moment $\mu_{\text {eff }}$. It decreases from $1.85 \mu_{B}$ at $x=0$ to $1.1 \mu_{B}$ at $x=0.55$. Remarkably, the observed Weiss temperatures $\left(\Theta_{W}\right)$ are very similar for all different investigated samples. For $x=0.55 \Theta_{W}=-25 \mathrm{~K}$, which is close to $-33 \mathrm{~K}$ for $x=0$. Hence the $\mathrm{CW}$ scale remains almost unchanged for more than $50 \%$ dilution of magnetic moments in the Li-system in stark contrast to its drastic reduction found for the Na-system. At low temperatures, a hysteresis between FC and ZFC susceptibility data is found, similar as for the Na-system. Fig. 5.22 shows a separation between the FC and ZFC susceptibility which confirms $T_{\mathrm{g}}=3.5 \mathrm{~K}$ and $2 \mathrm{~K}$ for $x=0.09$ and $x=0.22$, respectively (vertical arrows in Fig. 5.22 indicate $T_{\mathrm{g}}$ ). The ac susceptibility also shows a strong frequency dependence for these two compositions. Similar SG freezing behavior is also present at higher doping below the temperature limit of our SQUID magnetometer $(1.8 \mathrm{~K})$

Heat capacity: To investigate SG behavior for all $\mathrm{Li}_{2}\left(\mathrm{Ir}_{1-x} \mathrm{Ti}_{x}\right) \mathrm{O}_{3}$ samples the heat 


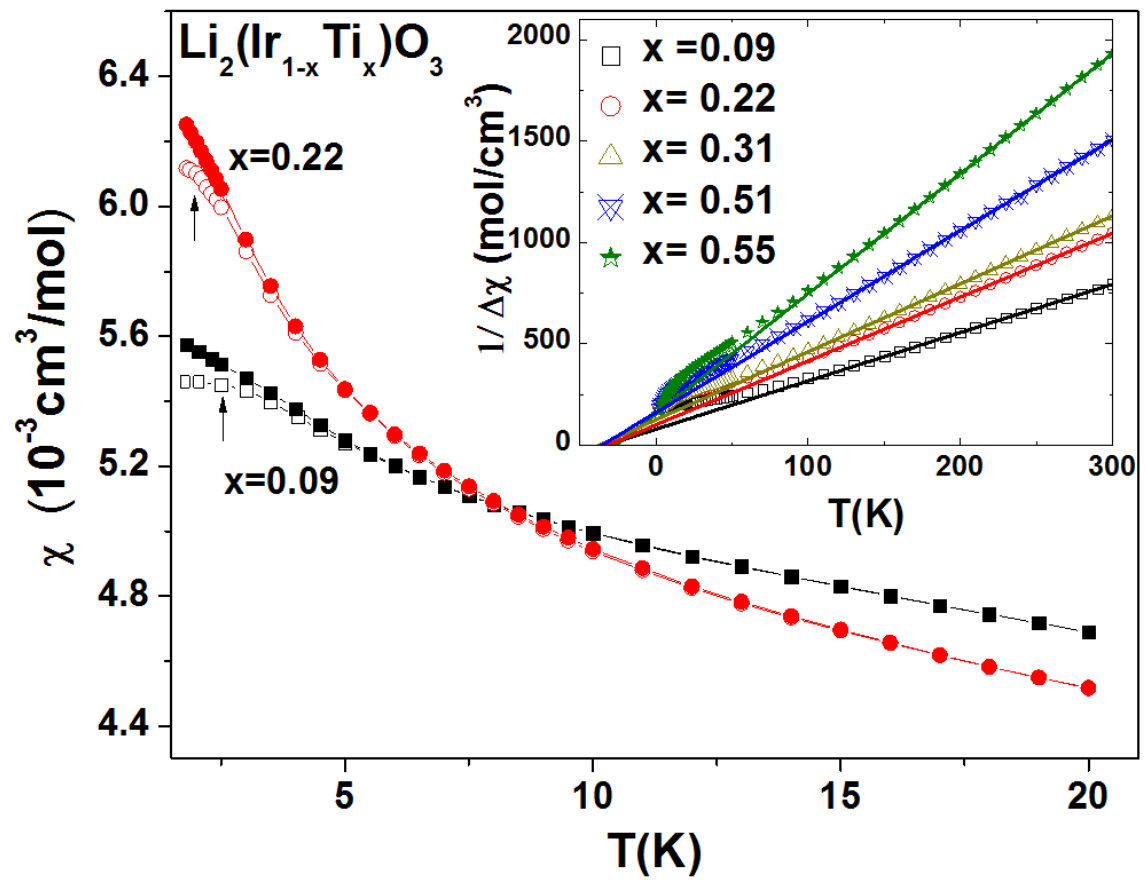

Figure 5.22: $\mathrm{FC}$ and ZFC susceptibility (represented by filled and open symbols, respectively) for $\operatorname{Li}_{2}\left(\operatorname{Ir}_{1-x} \mathrm{Ti}_{x}\right) \mathrm{O}_{3}$ with $x=0.09$ and 0.22 , measured at $H=0.01 \mathrm{~T}$. Vertical arrows mark $T_{\mathrm{g}}$. The inset displays $1 / \Delta \chi$ versus $T$ for all investigated $x$. Solid lines illustrate $\mathrm{CW}$ behavior. Reprinted from [81].

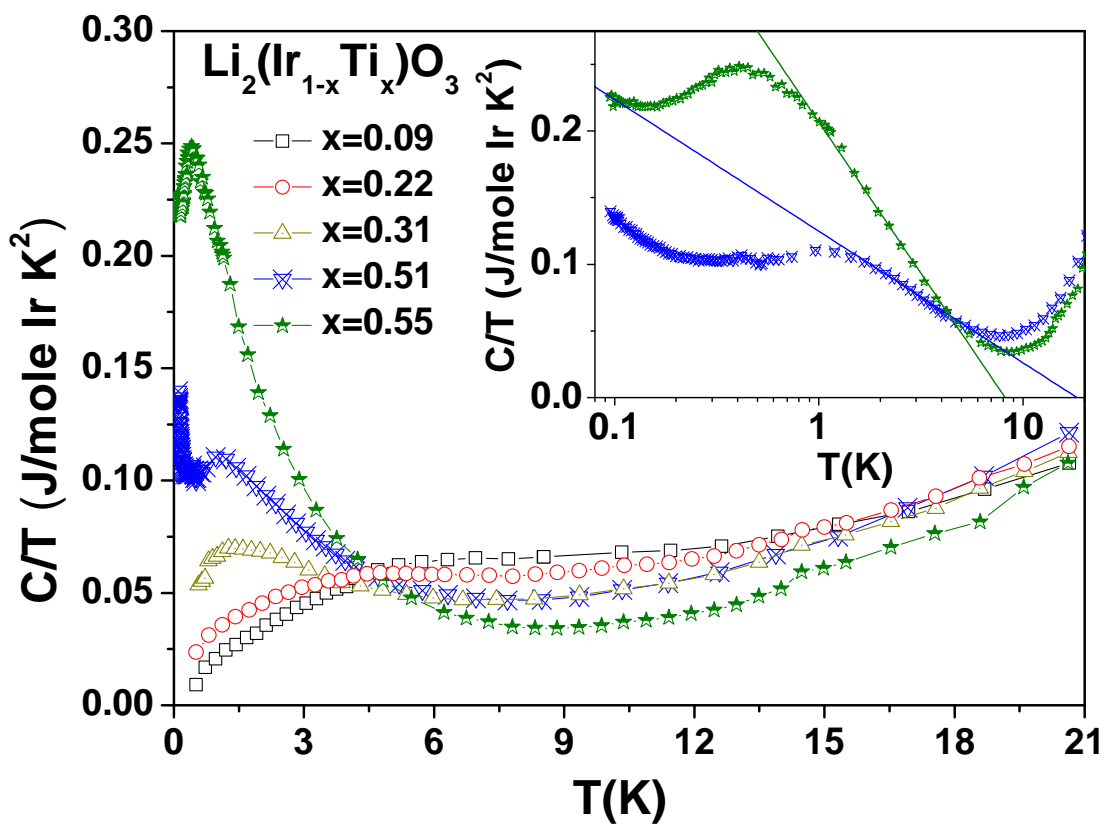

Figure 5.23: Specific heat as $C / T$ vs. $T$ for various $\operatorname{Li}_{2}\left(\operatorname{Ir}_{1-x} \mathrm{Ti}_{x}\right) \mathrm{O}_{3}$ samples. The inset displays the low- $T$ data for $x=0.51$ and 0.55 on a $\log (T)$ axis. Broad maxima indicate $T_{\mathrm{g}}$, above which a logarithmic temperature dependence is found (see lines). Reprinted from [81]. 
capacity was measured down to $0.4 \mathrm{~K}$ and extended the data down to $50 \mathrm{mK}$ for the two highest concentrations, cf. Fig. 5.23. For $x=0.09$ and 0.22 a broad maxima in heat capacity divided by temperature $C / T$ is obatined around $1.4 T_{g}$ which is characteristic for SG transitions (see Fig. 5.23). For $x=0.31,0.51$ and 0.55 similar broad maxima are found at low temperatures. With increasing $x$ the position of these maxima shift from $1.25 \mathrm{~K}$ to $0.41 \mathrm{~K}$ for $x=0.31$ to 0.55 . The respective $T_{\mathrm{g}}$ values are determined by the position of the maximum divided by 1.4. From Fig. 5.23 it is unambiguously clear that even beyond $50 \%$ substitution of magnetic Ir sites by non-magnetic Ti in the Li-honeycomb system SG freezing persists and $T_{\mathrm{g}}$ continuously shifts to lower temperatures with increasing $x$. Strikingly $C / T$ for $x=0.51$ and $x=0.55$ does not approach 0 at lowest temperatures as expected for insulators but rather saturates (above a low- $T$ nuclear upturn). This implies that a significant amount of magnetic entropy is present at lowest temperature.

As indicated by the straight lines in the inset of Fig. 5.23, a logarithmic increase of $C / T$ is found for $x=0.51$ and 0.55 upon cooling from about $8 \mathrm{~K}$ down to the SG freezing. Such behavior is often found near magnetic instabilities and considered as signature of quantum criticality. Also a strong non-monotonic field dependence of $C / T$ was observed for $x=0.51$ (Fig. 5.25) and 0.55 (Fig. 5.24). But above a certain filed this characteristic field dependence was totally destroyed rather shows typical Schottky anomaly resulted.

Thermal and magnetic Grüneisen parameter: ${ }^{5}$ The adiabatic magnetocaloric effect or magnetic Grüneisen parameter $\Gamma_{H}=T^{-1}(d T / d H)_{S}$ is a sensitive probe of quantum criticality and is expected to diverge as a function of temperature with a power-law function at the critical field $H_{c}$ for a field-induced quantum critical point (QCP) [85]. Fig. 5.24 displays the temperature dependence of $\Gamma_{H}$ at different magnetic fields for $\operatorname{Li}_{2}\left(\operatorname{Ir}_{1-x} \operatorname{Ti}_{x}\right) \mathrm{O}_{3}$, $\mathrm{x}=0.55$. At low field of $0.2 \mathrm{~T}$, a divergence with exponent of -1.7 is found over at least one decade in $T$, indicating quantum critical behavior with a low critical field. At $0.4 \mathrm{~T}$ and larger fields, $\Gamma_{H}(T)$ saturates upon cooling and the saturation temperature increases with increasing field indicating that fields drive the system away from quantum criticality. The data at various different fields collapse on a single curve when plotted as $\Gamma_{H} h$ vs. $T / h^{\epsilon}$ (see inset of lower panel of Fig. 5.24). Here $h$ denotes the difference in field from the critical field, i.e., $h=H-0.2 \mathrm{~T}$ and the scaling exponent amounts to $\epsilon=0.86$. The critical field of $0.2 \mathrm{~T}$ is consistent with the power-law divergence of $\Gamma_{H}(T)$ only observed at $0.2 \mathrm{~T}$. Furthermore, the non-monotonic field dependence of the low-temperature specific heat is probably due to the small finite $H_{c}$.

\footnotetext{
${ }^{5}$ All the measurements in the dilution fridge were done by Yoshi Tokiwa and Christian Stingl.
} 


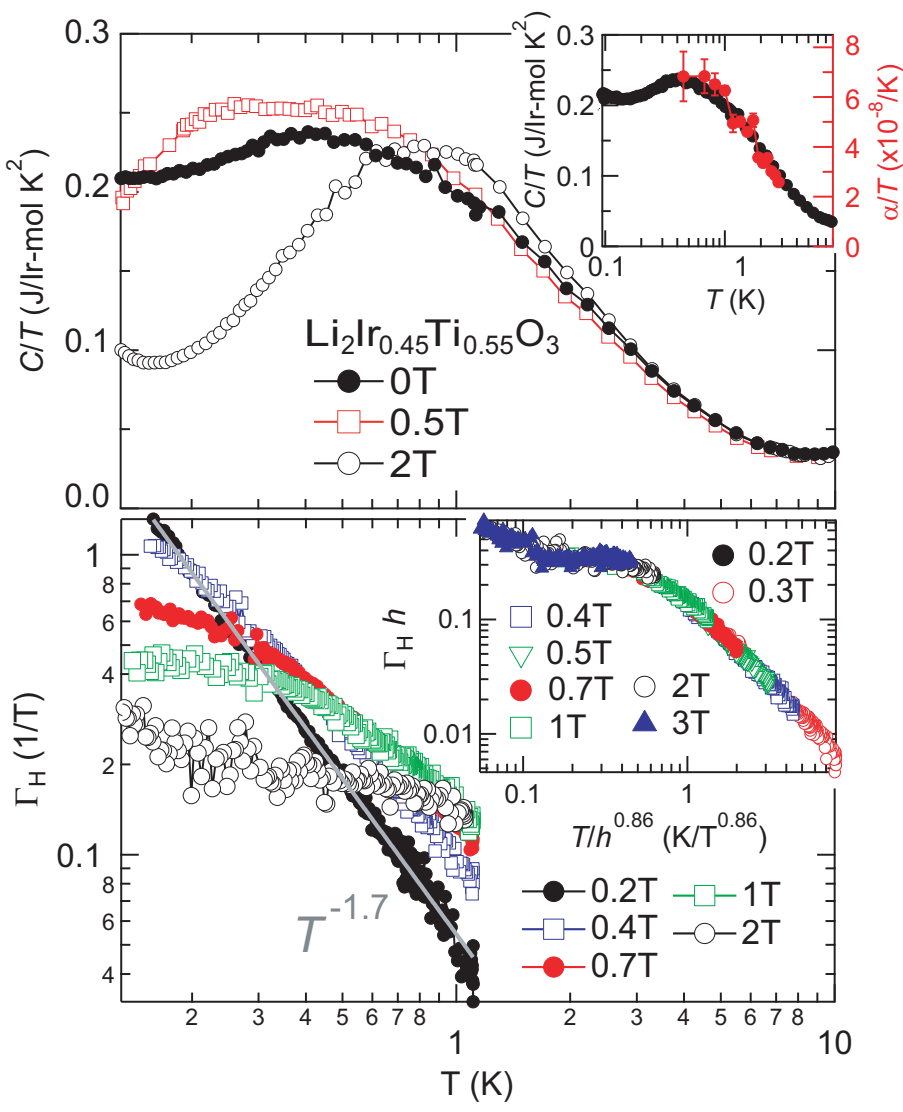

Figure 5.24: Upper panel: Specific heat as $C / T$ vs. $T$ (on $\log$ scale) at various fields for $\mathrm{Li}_{2}\left(\mathrm{Ir}_{1-x} \mathrm{Ti}_{x}\right) \mathrm{O}_{3}, \quad x=$ 0.55. The inset displays zero field data together with respective thermal expansion data as $\alpha / T$. Lower panel: Magnetic Grüneisen parameter $\Gamma_{H}=T^{-1}(d T / d H)_{S}$ at different magnetic fields vs. $T$ (on $\log$-log scale). The solid line indicates the $T^{-1.7}$ divergence at $0.2 \mathrm{~T}$. The inset displays scaling behavior, $\Gamma_{H} h$ vs $T / h^{\epsilon}$ with $\epsilon=0.86$ and $h=(H-0.2 \mathrm{~T})$. Reprinted from [81].

For $\operatorname{Li}_{2}\left(\operatorname{Ir}_{1-x} \mathrm{Ti}_{x}\right) \mathrm{O}_{3}, x=0.51$ the specific heat divided by temperature $C / T$ shows a similar non-monotonic (as for $x=0.55$ ) but stronger field dependence at low temperature. In Fig. 5.25 temperature dependence of $\Gamma_{H}$ at different magnetic fields is shown. $\Gamma_{H}$ at $0.2 \mathrm{~T}$ diverges as a function of temperature, indicating a presence of QCP very near to this magnetic field. At higher fields, $\Gamma_{H}$ saturates at low temperatures, suggesting that the system is driven away from QCP at fields above $0.2 \mathrm{~T}$ (lower plot in Fig. 5.25). Similar as for $x=0.55$, the $x=0.51-\Gamma_{H}$ data collapse in a common curve, when $\Gamma_{H} h$ is plotted against $T / h^{\epsilon}$ (lower inset Fig. 5.25 with $\epsilon=0.67$ and $H_{c}=0.24 \mathrm{~T}$. Hence field-tuned quantum criticality at $H_{c}=0.24 \mathrm{~T}$ is confirmed in the $x=0.51$ sample also.

To further characterize the low-temperature magnetic properties of depleted $\mathrm{Li}_{2}\left(\operatorname{Ir}_{1-x} \mathrm{Ti}_{x}\right) \mathrm{O}_{3}$, temperature dependence of the linear thermal expansion coefficient $\alpha(T)=L^{-1} d L / d T(L$ : sample length) was studied for $x=0.55$, see upper inset of Fig. 5.24. The large values of order $10^{-6} \mathrm{~K}^{-1}$ around $1 \mathrm{~K}$ must originate from the magnetic properties (the phonon contribution is several orders of magnitude smaller). Interestingly, $\alpha / T$ perfectly scales with $C / T$ indicating a temperature independent thermal Grüneisen ratio $\Gamma \sim \alpha / C$. This proves the absence of a QCP as function of pressure [85] and resembles the case of $\mathrm{CePd}_{1-x} \mathrm{Rh}_{x}$ 


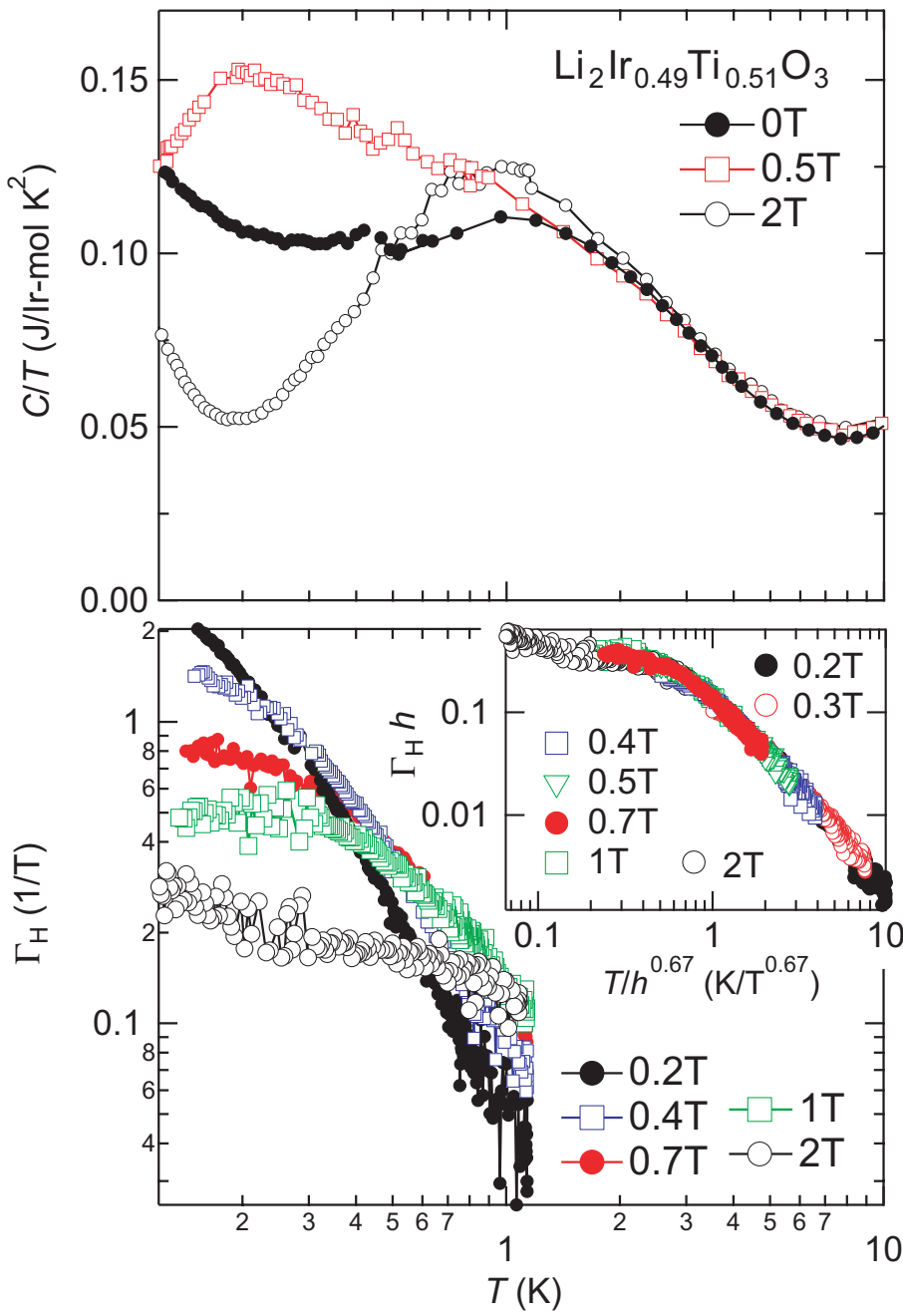

Figure 5.25: (Upper) variation of $C / T$ vs. $T$ for $x=$ $0.51, \mathrm{Li}_{2}\left(\mathrm{Ir}_{1-x} \mathrm{Ti}_{x}\right) \mathrm{O}_{3}$ at different $H$. Lower: Magnetic Grüneisen parameter $\Gamma_{H}$ vs. $T$ on $\log -\log$ scale, (inset) scaling,$\Gamma_{H} h$ vs $T / h^{\epsilon}$ plot, with $\epsilon=0.67$ and $h=(H-0.24) \mathrm{T}$. Reprinted from [81].

where $\Gamma(T)$ also does not diverge due to the smeared phase transition [86]. The observed entropy accumulation at low- $T$ which is quenched by a magnetic field but remains unaffected by pressure (or changes in composition) would then arise from weakly coupled magnetic clusters. Low-temperature experiments on $\mathrm{Li}_{2}\left(\mathrm{Ir}_{1-x} \mathrm{Ti}_{x}\right) \mathrm{O}_{3}$ thus prove that SG formation survives upon substantial magnetic depletion up to $x=0.55$ leading to a smeared QPT.

\subsubsection{Discussions}

The variation of the SG freezing temperatures for the depleted Na- and Li-systems is summarized in the upper panel of Fig. 5.26. [81] Normalized SG freezing temperature $T_{g}(x) / T_{g}(0)$ is plotted in Fig. 5.26, where $T_{\mathrm{g}}(0)$ is linear extrapolation of $T_{\mathrm{g}}(x)$ to $x=0$ axis. In both systems, already small magnetic depletion induces a SG transition, highlighting the importance of magnetic frustration, and the freezing temperature $T_{\mathrm{g}}$ displays a linear suppression at low Ti concentration $x$. However, the evolution of the Weiss temperature shown in the 


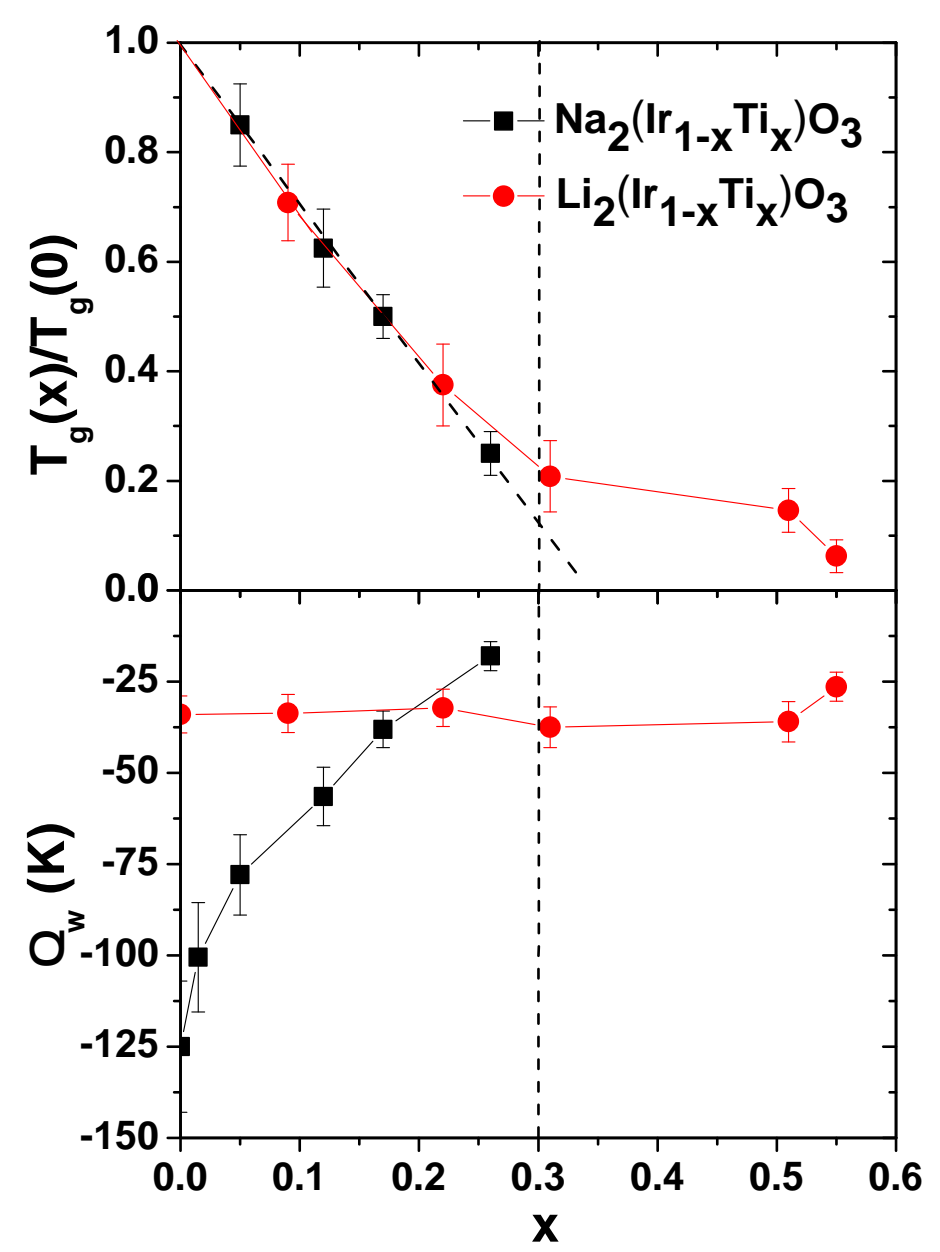

Figure 5.26: Evolution of normalized spin glass ordering temperatures (upper panel) and Curie Weiss temperatures (lower panel) for $\mathrm{Na}_{2}\left(\mathrm{Ir}_{1-x} \mathrm{Ti}_{x}\right) \mathrm{O}_{3}$ and $\mathrm{Li}_{2}\left(\mathrm{Ir}_{1-x} \mathrm{Ti}_{x}\right) \mathrm{O}_{3}$. The vertical dotted line at $x=0.3$ indicates the percolation threshold in the honeycomb lattice. The dashed black line in the upper panel indicates the linear suppression of $T_{\mathrm{g}}$ for $\mathrm{Na}_{2}\left(\mathrm{Ir}_{1-x} \mathrm{Ti}_{x}\right) \mathrm{O}_{3}$. Reprinted from [81].

lower panel indicates a substantially different response to magnetic depletion of the two systems. While for the Na-system a drastic reduction of $\left|\Theta_{W}\right|$ indicates a suppression of the average magnetic couplings by dilution, $\left|\Theta_{\mathrm{W}}\right|$ remains unchanged in case of the Li-system. In addition, for the Li-system the signatures of SG formation extend to large $x \sim 0.55$, where signatures of a smeared QPT are observed. Although we could not study $\mathrm{Na}_{2}\left(\operatorname{Ir}_{1-x} \mathrm{Ti}_{x}\right) \mathrm{O}_{3}$ at large $x$, the evolution of the magnetic coupling strength (from $\left|\Theta_{\mathrm{W}}\right|$ ) suggests that the QPT for this system is located at significantly lower $x$. Eric et. al. by classical Monte-Carlo simulations on depleted next neighbor HK and $J_{1^{-}} J_{2^{-}} J_{3}$ Heisenberg models found that in the former case SG freezing disappears beyond the site percolation threshold $x_{p}=0.3$ while in the latter case with substantial further neighbor couplings it persists much beyond $x_{p}$ [83]. Comparison with our data suggests that $\mathrm{Na}_{2} \mathrm{IrO}_{3}$ is governed dominantly by the nearest neighbor HK model whereas for the Li-system interactions beyond nearest neighbor are significantly important. Interestingly, $x=0.50$ is the site percolation threshold for a triangular lattice and for $J_{2}$ exchange only, the honeycomb system corresponds to two decoupled triangular lattices. Thus, the observed smeared QPT must be associated with further neighbor 
interactions. We also note, that recent theoretical work related to $\mathrm{Li}_{2} \mathrm{IrO}_{3}$ found that the low- $Q$ spiral ordering in combination with the AF Weiss temperature $\Theta_{W}=-30 \mathrm{~K}$ requires a model with second neighbor Kitaev and Heisenberg interactions [35].

To summarize, a very different behavior in depleted honeycomb $\mathrm{Na}_{2} \mathrm{IrO}_{3}$ and $\mathrm{Li}_{2} \mathrm{IrO}_{3}$ suggests the importance of substantial further neighbor magnetic interactions for $\mathrm{Li}_{2} \mathrm{IrO}_{3}$. In $\mathrm{Li}_{2}\left(\mathrm{Ir}_{1-x} \mathrm{Ti}_{x}\right) \mathrm{O}_{3}$, SG freezing persists to a regime at $x \sim 0.55$ for which indications of a smeared quantum phase transition is observed. Magnetism in this interesting regime could be further investigated by NMR, $\mu \mathrm{SR}$ or neutron scattering. $\mathrm{Na}_{2} \mathrm{IrO}_{3}$ magnetism is almost destroyed by non-magnetic dilution even below percolation threshold, it clearly identifies that $\mathrm{Na}_{2} \mathrm{IrO}_{3}$ magnetic exchange is solely governed by nearest neighbor interactions (e.g. the Heisenberg and Kitaev interaction). 



\section{Chapter 6}

\section{Discussion of the $\mathrm{A}_{2} \mathrm{IrO}_{3}$ results on the context of Heisenberg-Kitaev Physics}

Structural, magnetic and thermodynamic bulk investigation and microscopic investigation by neutron scattering, as well as nonmagnetic dilution have clearly identified the magnetic structure and provided preliminary information on the magnetic interaction strengths of both $\mathrm{Na}_{2} \mathrm{IrO}_{3}$ and $\mathrm{Li}_{2} \mathrm{IrO}_{3}$. In particular, we have found:

- $\mathrm{Na}_{2} \mathrm{IrO}_{3}$ has a zigzag magnetic ground state below $T_{N}=15 \mathrm{~K}$ and strong AF CW scale $\left(\theta_{W}=-125 \mathrm{~K}\right)$ which both can be destroyed by tiny nonmagnetic dilution. This suggests that $\mathrm{Na}_{2} \mathrm{IrO}_{3}$ magnetism is mainly governed by nearest-neighbor frustrated exchange interaction.

- $\mathrm{Li}_{2} \mathrm{IrO}_{3}$ displays an incommensurate spiral magnetic structure below $T_{N}=15 \mathrm{~K}$ and weaker AF CW scale compared to the Na-system $\left(\theta_{W}=-40 \mathrm{~K}\right)$ which persist even beyond $50 \%$ nonmagnetic dilution of its magnetic site. This clearly points to the importance of beyond nearest-neighbor frustrated interactions in $\mathrm{Li}_{2} \mathrm{IrO}_{3}$.

\section{1 $\quad \mathrm{Na}_{2} \mathrm{IrO}_{3}$}

The only feasible nearest neighbor spin-model for honeycomb lattice iridates is NN HeisenbergKitaev (HK) model described in Eqn. 2.8 - 2.11. Here the dynamical frustration of anisotropic 

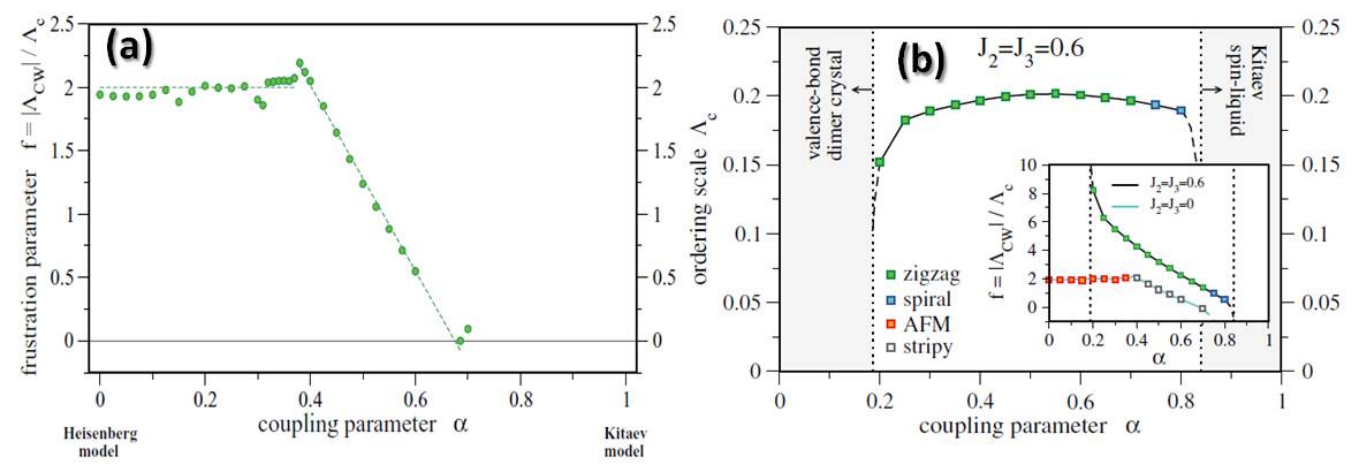

Figure 6.1: (a) Frustration parameter $(f)$ determined from the ratio of the CW scale $\Lambda_{C W}$ to the ordering scale $\Lambda_{c}$ calculated by pseudo fermion functional renormalization group flow (PFFRG) using the NN HK model(Eqn. 2.9), reprinted from [87]. (b) Ordering scale $\Lambda_{c}$ calculated by PFFRG for the $J_{2}-J_{3}$ HK model as function of $\alpha$. Inset shows frustration parameter $f$ vss. $\alpha$, reprinted from [60].

Kitaev interaction and competition of the NN Heisenberg and Kitaev exchange help to realize novel unconventional magnetic structures. But zigzag magnetic structure of $\mathrm{Na}_{2} \mathrm{IrO}_{3}$ is not described in the first proposed form of HK model (Eqn. 2.8 - 2.9) where the Kitaev interaction is $\mathrm{FM}(K<0)$ and the Heisenberg one is $\mathrm{AF}(J>0)$. The only unconventional magnetic structure of this model is stripy, shown in the phase diagram [Fig. 2.7(a)].[13] The difference between the stripy and the zigzag phase is that the zigzag spin structure has antiferromagnetically coupled chains of ferromagnetically coupled spins while stripy one has ferromagnetically coupled chains of antiferromagnetically coupled spins. Hence magnetic ground state of $\mathrm{Na}_{2} \mathrm{IrO}_{3}$ can not be explained by the HK model with FM Kitaev exchange.

The finite-temperature phase diagram of the HK model (Eqn. 2.8) was investigated by the group of Simon Trebst. They have developed pseudofermion functional renormalization group (PFFRG) approach to compute the magnetic susceptibility from the pseudofermion two-particle vertex function evolving under a RG flow with a frequency cutoff $\Lambda$. [87] They proposed to extract high-temperature $\mathrm{CW}$ behavior from the RG flow, the onset of magnetic ordering (from the breakdown of the RG flow), and momentum-resolved magnetic susceptibility profiles, which also allow to identify the nature of the various ground states of model. The RG flow was fitted by CW behavior:

$$
\chi=C /\left(\Lambda-\Lambda_{C W}\right)
$$

where the CW scale $\Lambda_{C W}$ was obtained from the fitting of the RG flow by Eqn. 6.1 at the onset of magnetic ordering. Ordering scale $\Lambda_{C}$ for a particular $\alpha$ is the frequency $(\Lambda)$ where the RG flow breaks down. Frustration parameter $(f)$ is determined by $\left|\Lambda_{C W}\right| / \Lambda_{C}$. This is 

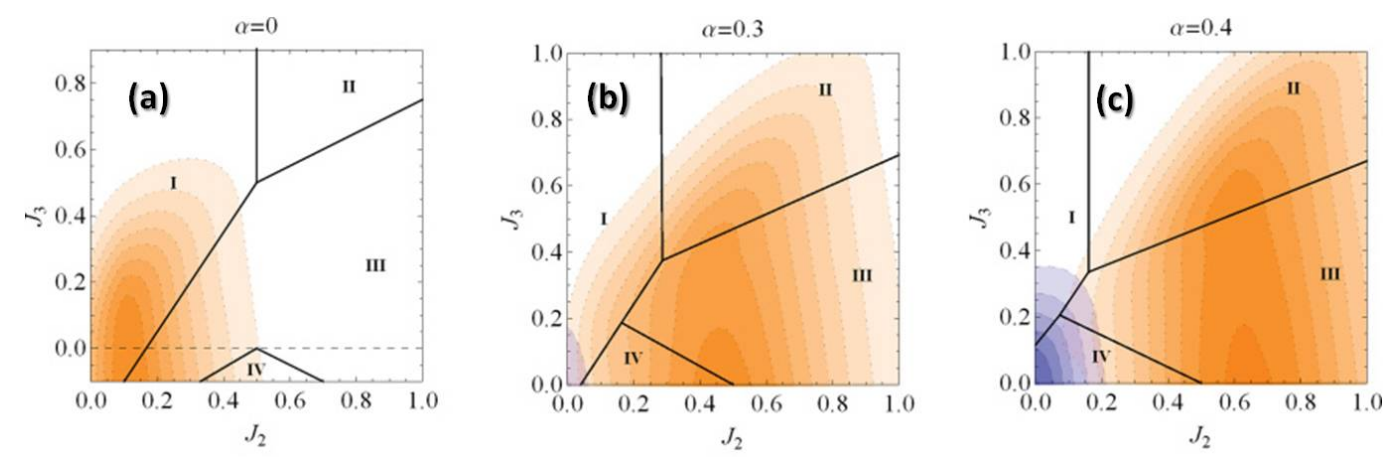

Figure 6.2: (a-c) Fixed $\alpha$ slices in $\left(J_{2}, J_{3}\right)$ showing the magnetically ordered phases (I, II, III, IV $)=\left(\right.$ Néel, zigzag, spiral, stripy) for $\alpha=0,0.3,0.4$ respectively, obtained from $J_{2}-J_{3}$ HK model. Orange and Blue shade corresponding to the exact diagonalization (ED) $\chi(T)$ fit goodness for $\mathrm{Na}_{2} \mathrm{IrO}_{3}$ and $\mathrm{Li}_{2} \mathrm{IrO}_{3}$ respectively. Reprinted from [34].

plotted for nearest neighbor (NN) HK model with FM $K$ in Fig. 6.1 (a). Maximum value of $f$ for the HK model is 2 but for $\mathrm{Na}_{2} \mathrm{IrO}_{3}$ the experimental frustration parameter is much larger, $f=\left|\theta_{W}\right| / T_{N}=125 / 15 \approx 8$. Hence the finite temperature phase diagram for the NN HK model also does not match with the experimental data for $\mathrm{Na}_{2} \mathrm{IrO}_{3}$.

The zigzag magnetic ground state of $\mathrm{Na}_{2} \mathrm{IrO}_{3}$ was first explained only after inclusion of long ranged AF Heisenberg interactions.[34, 36, 60] Inelastic neutron scattering data and the boundary of the magnon dispersion of $\mathrm{Na}_{2} \mathrm{IrO}_{3}$ was fitted by $J_{1}-J_{2}-J_{3}$ model (Eqn. 2.12) In the zigzag spin structure domain, $J_{1}=4.17 \mathrm{meV}, J_{2} / J_{1}=0.78 \mathrm{meV}, J_{3} / J_{1}$ $=0.9$. [36] These values for the exchange interactions reproduce the $\mathrm{CW}$ temperature, $\theta_{W}=$ $-S(S+1)\left(J_{1}+2 J_{2}+J_{3}\right) / k_{B} \approx-125 \mathrm{~K}$. They have analyzed the spin-wave spectra with $J_{2}-J_{3} \mathrm{HK}$ model and concluded that if at all any Kitaev exchange in $\mathrm{Na}_{2} \mathrm{IrO}_{3}$ it is very small, i.e. $\alpha \leq 0.40$. Their main description of $\mathrm{Na}_{2} \mathrm{IrO}_{3}$ was by isotropic $J_{1}-J_{2}-J_{3}$ model and they concluded Kitaev exchange was not necessary for $\mathrm{Na}_{2} \mathrm{IrO}_{3}$.

A detail description of the $J_{2}-J_{3}$ HK model was first done by Kimchi et. al. [34] They obtained the ordered phase diagram for fixed alpha values in the $\left(J_{2}-J_{3}\right)$ parameter space, see in Fig. 6.2 where it is shown for $\alpha=0,0.3,0.4$. They have compared thermodynamic susceptibility data to the exact diagonalization (ED) numerical results. The "goodness" of the ED and experimental $\chi(T)$ data is plotted in color. Strongest intensity of a color represent best fit. Orange color represents $\mathrm{Na}_{2} \mathrm{IrO}_{3}$ data. Hence good fit in the zigzag phase [II in Fig. 6.2 (b-c)] is obtained for large value of $J_{2}$ and $J_{3}$ and $\alpha \neq 0$. The determined range for the $\mathrm{Na}_{2} \mathrm{IrO}_{3}$ zigzag phase is: $0.25 \leq \alpha \leq 0.5, J_{2} \geq 0.3$ and $J_{3} \geq 0.4$. This study also clearly demanded a substantial Heisenberg exchange beyond next neighbor to describe the magnetism of $\mathrm{Na}_{2} \mathrm{IrO}_{3}$. 
The finite-temperature phase diagram was studied by PFFRG approach for the $J_{2}-J_{3}$ HK model as well.[60] Fig. 6.1 (b) shows the variation of the ordering scale $\Lambda_{c}$ and the frustration parameter $f$ vs. $\alpha$ for representative values $J_{2}=J_{3}=0.6$. For $\alpha$ ranging from 0.3 to 0.8 the ordering scale is almost constant. In this range the magnetic ground state is also zigzag (except spiral at $\alpha=0.7$ and 0.8 ). The frustration parameter $f$ for $\alpha=0.2$ (at the lower boundary of the zigzag phase) is 8 which is very similar to the experimental value for $\mathrm{Na}_{2} \mathrm{IrO}_{3}$. Hence this study also suggests that a substantial amount of $J_{2}$ and $J_{3}$ included to the NN HK model can actually describe the zigzag magnetic ground state of $\mathrm{Na}_{2} \mathrm{IrO}_{3}$ much easily.

A theoretical mapping of the experimental data for $\mathrm{Na}_{2} \mathrm{IrO}_{3}$ is demanding substantial further neighbor Heisenberg exchange. Whereas a huge $3^{r d}$ neighbor exchange $\left(J_{3} / J_{1}=0.9\right)$ is very much contradictory to the ab-initio calculations for the $\mathrm{Na}_{2} \mathrm{IrO}_{3}$ where $2^{\text {nd }}$ neighbor exchange is also assumed to be very small.[88] Nearest neighbor hoppings are the most important hopping terms for $\mathrm{Na}_{2} \mathrm{IrO}_{3}$ calculated from ab-intio calculation. nonmagnetic dilution clearly shows that the magnetic exchange in $\mathrm{Na}_{2} \mathrm{IrO}_{3}$ is almost destroyed near the percolation threshold for the NN exchange $\left(x_{p}=0.303\right)$. Therefore a huge $\mathbf{2}^{\text {nd }}$ and $\mathbf{3}^{\text {rd }}$ neighbor exchange $J_{2}$ and $J_{3}$ for $\mathrm{Na}_{2} \mathrm{IrO}_{3}$ has to be excluded.

First time a nearest neighbor model which described zigzag phase in honeycomb lattice iridate was generalized HK model given in Eqn. 2.10 and 2.11.[33] The phase diagram in Fig. 2.7(b) shows that a zigzag phase is observed in the region $\pi \leq \varphi \leq \pi / 2$ where a interorbital $t_{2 g}-e_{g}$ hopping process gives rise to AF NN Kitaev exchange and FM NN Heisenberg exchange. The excitation energy for this $t_{2 g}-e_{g}$ hopping is $\tilde{U}=U+10 D q$ where $10 D q$ is the crystal-field splitting. Recently an ab-intio study was done on $\mathrm{Na}_{2} \mathrm{IrO}_{3}$ with the QMO and NN HK model.[88] The hopping parameters for all the four hopping processes were calculated by DFT. Then a $\tilde{U}$ vs $U$ phase diagram was drawn Having $K / J$ parameter sapce.[88] Zigzag phase does not appear in the physical parameter range of $U$ and $\tilde{U}$. Also in zigzag region $\tilde{U}<0.6 U$, i.e the Hubbard gap is larger than the $t_{2 g}-e_{g}$ splitting, a very unlikely situation. Physical range of parameters for $\mathrm{Na}_{2} \mathrm{IrO}_{3}$ is ferromagnetic or spin-liquid. The reported AF Kitaev term and FM Heisenberg term for $\mathrm{Na}_{2} \mathrm{IrO}_{3}$ are $K=10.44 \mathrm{meV}$ and $J=-4.01 \mathrm{meV}$ respectively which was claimed unrealistic from ab-intio calculation.[88] Hence this nearest neighbor scenario is also unrealistic for $\mathrm{Na}_{2} \mathrm{IrO}_{3}$.

Very recently another ab-initio calculation was done for $\mathrm{Na}_{2} \mathrm{IrO}_{3}$.[89] For the first time, the trigonal distortion of the $\mathrm{IrO}_{6}$ octahedra was taken into account. The $t_{2} g$ Hamiltonian was described as:

$$
\hat{H_{t 2 g}}=\hat{H_{0}}+\hat{H_{t r i}}+\hat{H_{S O C}}+\hat{H_{U}}
$$


Where $\hat{H}_{0}$ is the hopping term describing mainly nearest neighbor hoppings, $\hat{H_{t r i}}$ describes the onsite trigonal distortion term $\Delta, \hat{H_{S O C}}$ is the spin-orbit coupling Hamiltonian described by $\lambda_{S O}$ and $\hat{H}_{U}$ is the Coulomb repulsion. The tight-binding parameters were obtained,[89] $\Delta=-28 \mathrm{meV}$ seems very tiny but this parameter actually generates relevant anisotropy. In the strong coupling limit the ab initio parameters for the generalized Kitaev-Heisenberg model (Eqn. 1 in Ref [89] including all exchange in NN) are derived from $t_{2 g}$ Hamiltonian $\hat{H_{t 2 g}}$ in Eqn. 6.2 by the second order perturbation theory. In the Strong SOC limit under small $\Delta$, lowest Kramers doublet is $J_{\text {eff }}=1 / 2$ states. Two anisotropic parameters $I_{1}$ and $I_{2}$ are obtained with AF $J$ and FM $K$. The generalized HK model were solved by exact diagonalization for a 24 -site cluster with the ab-initio parameters.

The derived exchange couplings stabilize zigzag phase which is interpreted as follows: If one assume the magnetic ordered moment along $(x, y, z)=(1,1,0)$, the zigzag order is interpreted as ferromagnetically-ordered chains consisting of the $X$ - and $Y$-bonds (stabilized by $K$ and $I_{2}$ ), antiferromagnetically coupled each other by the $Z$-bonds with $J$. The values of exchange constants are: $K=-30.1 \mathrm{meV}, J=4.4 \mathrm{meV}, I_{1}=-0.4 \mathrm{meV}$ and $I_{2}=1.1 \mathrm{meV}$. The finite temperature magnetic property are also nicely compared with experiment. Anisotropic magnetic susceptibility $\left(\chi_{>} \chi_{a b}\right)$ and the heat capacity of $\mathrm{Na}_{2} \mathrm{IrO}_{3}$ are fairly reproduced from this effective spin model with the derived exchange couplings.[89] Hence so far the most feasible spin-Hamiltonian for $\mathrm{Na}_{2} \mathrm{IrO}_{3}$ is this NN HK Hamiltonian with two anisotropic terms generated from trigonal distortion. Additionally a phase digram of all exchange couplings and magnetic ground state is presented with varying trigonal distortion $\Delta$. It is proposed that reducing trigonal distortion from -30 $\mathrm{meV}$ in $\mathrm{Na}_{2} \mathrm{IrO}_{3}$ one can achieve spin-liquid state near $\Delta=-5$ to $+20 \mathrm{meV}$.[89]

\section{2 $\quad \mathrm{Li}_{2} \mathrm{IrO}_{3}$}

The observed incommensurate spiral ordering in honeycomb lattice iridate $\mathrm{Li}_{2} \mathrm{IrO}_{3}$ seems impossible to be described by the Heisenberg-Kitaev physics. The nearest neighbor HK model with FM Kitaev exchange does not have any incommensurate phase with Bragg peak within first Brillouin zone (BZ). So this model can be clearly discarded for $\mathrm{Li}_{2} \mathrm{IrO}_{3}$. A generic spin model was derived in the $0 \leq \varphi \leq 2 \pi$ parameter space for the HK model including an anisotropic exchange parameter $\Gamma$ by Rau et. al. For certain $\Gamma>0$ their model hold a $120^{\circ}$ incommensurate phase for FM $J$ and $K$. But the ab-initio study by Foyevtsova et. al. already pointed out very unfeasible parameter space of Coulomb repulsion and CF splitting 


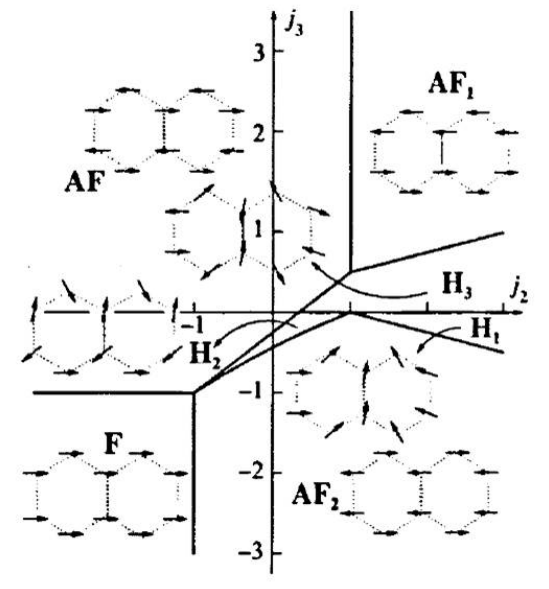

Figure 6.3:

Phase diagram

for Heisenberg

$J_{1}-J_{2} J_{3}$ model where $j_{3}=J_{3} / J_{1}$ and $j_{2}=J_{2} / J_{1}$ with $J_{1}>0$. Reprinted from $[37,38]$.

in zigzag phase of $\mathrm{Na}_{2} \mathrm{IrO}_{3}$.[88] Also a nonmagnetic dilution study on $\mathrm{Li}_{2} \mathrm{IrO}_{3}$ very categorically shown persistence of magnetic exchange much beyond NN percolation threshold. Thus a NN description of the magnetic exchange for $\mathrm{Li}_{2} \mathrm{IrO}_{3}$ is totally incomplete.

The earliest classical long ranged spin-model for honeycomb lattice is $J_{1}-J_{2}-J_{3}$ model (Eqn. 2.12). In Fig. 6.3 phase diagram for this model in $j_{3}-j_{2}$ phase space $\left(j_{3}=J_{3} / J_{1}\right.$ and $\left.j_{2}=J_{2} / J_{1}\right)$ is shown. $H_{1}, \mathrm{H}_{2}$ and $\mathrm{H}_{3}$ are the three noncollinear phase exists in the phase diagram. Any of these phases can match with the spiral phase of $\mathrm{Li}_{2} \mathrm{IrO}_{3}$ depending on the $Q$-value of those phase and matching of Weiss temperate $\theta_{W}=-S(S+1)\left(J_{1}+2 J_{2}+J_{3}\right) / k_{B}$ calculated with $\theta_{W}=-40 \mathrm{~K}$ of $\mathrm{Li}_{2} \mathrm{IrO}_{3}$.

But if one carefully check structural differences between $\mathrm{Na}_{2} \mathrm{IrO}_{3}$ and $\mathrm{Li}_{2} \mathrm{IrO}_{3}$, the IrO-Ir bonding angle is more near to $90^{\circ}$ in the later, hence trigonal distortion is reduced in the later compared to the former by the effective $c$-axis pressure. So one would expect an enhanced FM Kitaev exchange.[60] For NN HK model it was predicted in the stripy phase $\chi(T \rightarrow 0) \propto 1 /(1-\alpha)$.[90] $\chi(T \rightarrow 0)$ for Li-system is two times larger than Na-system (see Fig. 4.8, 4.11). One would expect a larger $\alpha$ and a larger FM Kitaev interaction in the Li-system. Definitely one can not neglect the Kitaev term in $\mathrm{Li}_{2} \mathrm{IrO}_{3}$.

$\mathrm{Li}_{2} \mathrm{IrO}_{3}$ was analyzed with the $J_{2}-J_{3} \mathrm{HK}$ model and comparing its magnetic susceptibility with the theoretically obtained susceptibility from the model, the ground state of $\mathrm{Li}_{2} \mathrm{IrO}_{3}$ was also predicted as zigzag. [34, 60] ED study on the $J_{2}-J_{3}$ HK model for $\alpha=0.5$, 0.6 and 0.7 is shown in Fig. 6.4 (a-c) respectively. Blue shade represents the goodness of the fit of the ED $\chi$ to the measured $\chi$. For all the three $\alpha$-value good fit regions are observed in $\mathrm{Li}_{2} \mathrm{IrO}_{3}$. For the best fit $J_{2}>J_{3}$, atleast in the spiral phase region (III in Fig. 6.4 b,c) ${ }^{1}$

\footnotetext{
${ }^{1}$ For Honeycomb lattice iridates it is seen from ab-initio calculations of $\mathrm{Na}_{2} \mathrm{IrO}_{3}$ longer range hopping term has smaller values, so longer range Heisenberg superexchanges will also have smaller value. $J_{2}>J_{3}$ is more feasible in $\mathrm{Li}_{2} \mathrm{IrO}_{3}$.
} 

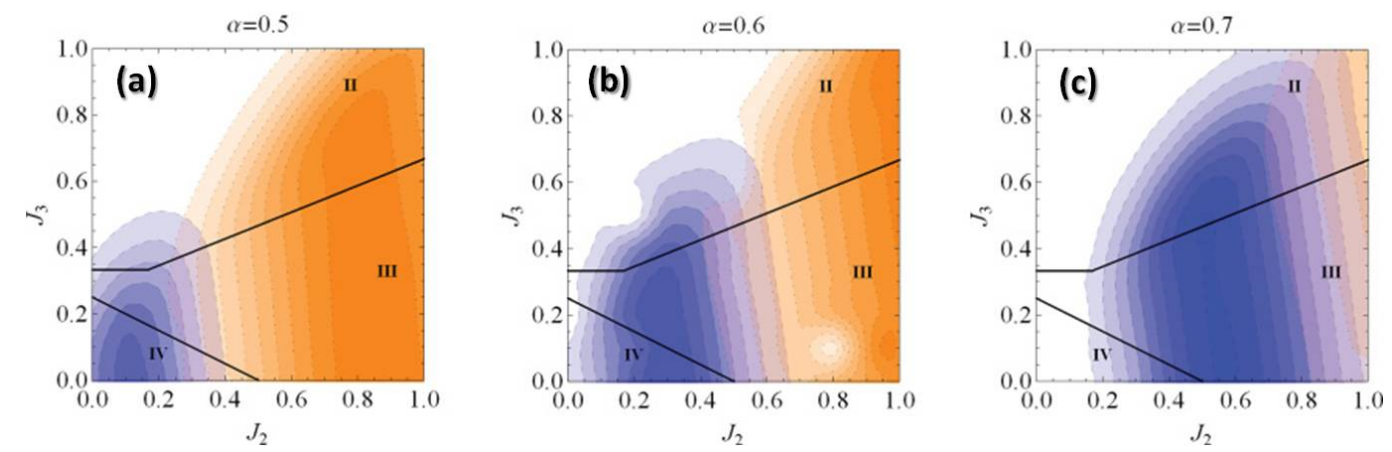

Figure 6.4: (a-c) Fixed $\alpha$ slices in $\left(J_{2}, J_{3}\right)$ showing the magnetically ordered phases (I, II, III, IV $)=\left(\right.$ Néel, zigzag, spiral, stripy) for $\alpha=0,0.3,0.4$ respectively, obtained from $J_{2}-J_{3}$ HK model . Orange and Blue shade corresponding to the exact diagonalization (ED) $\chi(T)$ fit goodness for $\mathrm{Na}_{2} \mathrm{IrO}_{3}$ and $\mathrm{Li}_{2} \mathrm{IrO}_{3}$ respectively. Reprinted from [34].

So a closer look in this phase diagram indicates spiral phase for $\mathrm{Li}_{2} \mathrm{IrO}_{3}$.

The PFFRG phase diagram shown in Fig. 6.1(b) for $J_{2}-J_{3}$ HK model with $J_{2}=J_{3}=$ 0.6. The inset shows that the frustration parameter $f$ drops from 8 to 2 for $\alpha=0.2$ to 0.7 . This change in $f$ is observed in the Na- to Li-system experimental value also, for Li-system, $f=\left|\theta_{W}\right| / T_{N}=40 / 15 \approx 2$. In this whole region the ordering scale is constant, for both Na- and Li- system $T_{N}$ is $15 \mathrm{~K}$. Near $\alpha=0.7$ magnetic ground state is also spiral [marked by blue square in the phase diagram Fig. 6.1(b)]. It seems beyond nearest neighbor model $J_{2}-J_{3} \mathrm{HK}$ model can well describe $\mathrm{Li}_{2} \mathrm{IrO}_{3}$ magnetic ground state and high temperature magnetic susceptibility. But logically there must be anisotropic NNN exchange if there is a isotropic one.

Shitade et. al showed that NNN hopping between isospins must have significant anisotropic term. Any superexchange between NNN Ir isospins has to be via anisotrpic Ir-O bonding which will lead to a anisotropic superexchange. Reuther et. al. derived an extended HK model with FM NNN isotropic Heisenber exchange and AFM anisotrpic Kitaev exchange given in Eqn. 2.15. To generate a phase diagram all the couplings are parametrized : $J_{1}$ $=\cos \left(\pi \phi_{1} / 2\right), K_{1}=-\sin \left(\pi \phi_{1} / 2\right), J_{2}=-\mathrm{g} \cos \left(\pi \phi_{2} / 2\right), K_{2}=\mathrm{g} \sin \left(\pi \phi_{2} / 2\right)$ with $\phi_{1,2} \in[0$, $1]$ and $g \geq 0 . \phi_{1(2)}$ changes the relative strength of Heisenberg and Kitaev interactions for (next) nearest neighbor couplings. Furthermore, $g$ is the total relative strength of first and second neighbor exchange. They have investigated this parametrized model using the pseudo fermion functional renormalization group (PFFRG) technique to estimate susceptibility profile as well as magnetic ordering. The resulting phase diagram as a function of $\phi_{1} \in[0,1]$ and $\phi_{2} \in[0,1]$ is shown in Fig. 6.5. Four magnetically ordered phases: FM order, AFM order, incommensurate spiral order with wave vectors outside the first Brillouin 

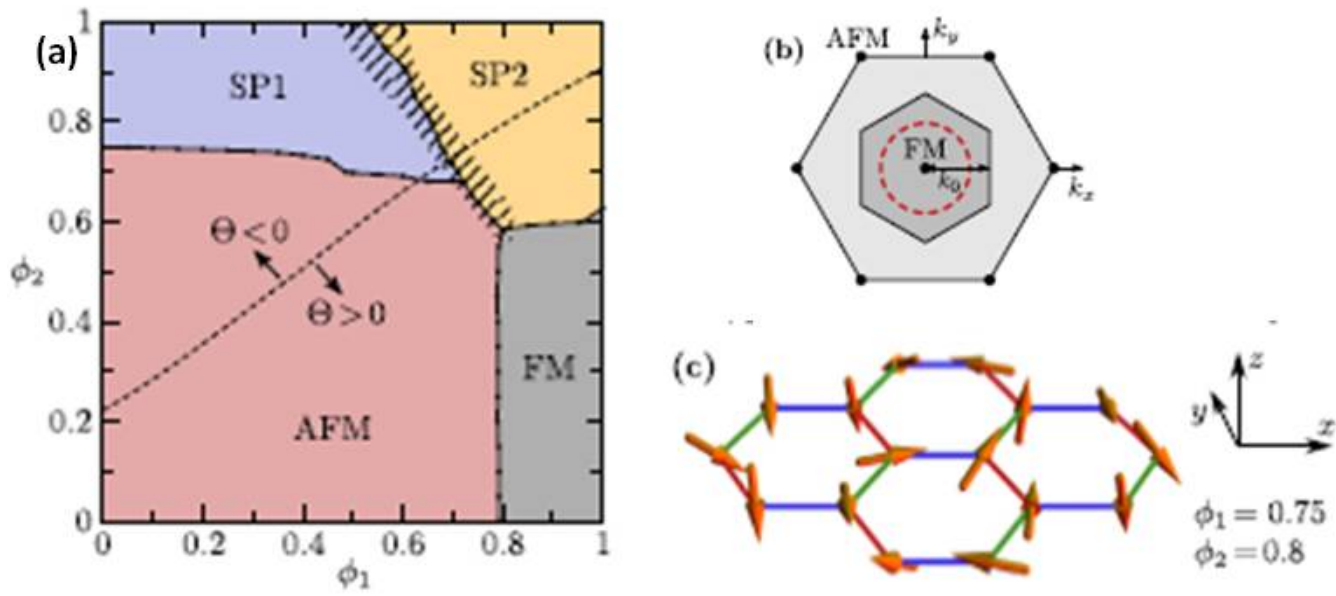

Figure 6.5: (a) Phase diagram for extended HK model reresented in Eqn. 2.15. Four magnetically ordered phases. Shaded areas indicate enhanced quantum fuctuations, possibly signaling a narrow non-magnetic phase. The dashed line separates parameter regimes with positive from negative CW temperature. (b) Mapping of the $q$-value in the spiral ordering phase SP2 in the reciprocal space of honeycomb iridate. (c) Possible noplanner spiral ordering for $\mathrm{Li}_{2} \mathrm{IrO}_{3}$. Reprinted from [35].

zone (SP1) and incommensurate spiral order with wave vectors inside the first Brillouin zone (SP2).[35]

For the SP1 phase the Weiss temperature $\Theta$ was found to be negative. But a wave vector within first $\mathrm{BZ}$ and negative Weiss temperature is not very common. $\mathrm{Li}_{2} \mathrm{IrO}_{3}$ is one of those rare real examples. It happens in this model only when value of $\phi_{1}$ and $\phi_{2}$ is significantly high i.e: both NN and NNN anisotropic Kitaev exchange are very high. Reuther et. al. investigated the susceptibility profile of this model with high NNN Kitaev exchange $\phi_{2}=0.8$ . It has been shown for $\phi_{1} \geq 0.65$ that a peak appears within first BZ in the susceptibility profile. Though a shoulder is always present outside the first BZ due to AFM NNN Kitaev exchange. This has not been observed so far in the Neutron scattering experiment of $\mathrm{Li}_{2} \mathrm{IrO}_{3}$. But in the SP2 phase the Bragg peak position [Fig. 6.5(b)] very clearly matches with the observed Bragg peak for $\mathrm{Li}_{2} \mathrm{IrO}_{3}$ [Fig. 4.23(c)]. Hence the HK model with NN AF Heisenberg, FM Kitaev, NNN FM Heisenberg and AF Kitaev exchange can be concluded as ideal description for $\mathrm{Li}_{2} \mathrm{IrO}_{3}$ magnetic exchange. The intrinsic relation between the real space and spin space transformations in the Kitaev model further requires that the $x-, y-, z-$ components of the real space spin-spin correlation function are rotated by $120^{\circ}$ among each other. This condition can only be fulfilled by a nonplanar spiral as shown in Fig. 6.5(c).[35] 


\section{Chapter 7}

\section{Honeycomb lattice Rhodate: $\mathrm{Li}_{2} \mathrm{RhO}_{3}$}

Honeycomb lattice iridates have been realized as novel spin-orbit Mott insulator where spin-orbit coupling (SOC) plays the most crucial role. The importance of SOC is also observed in other spin-orbit Mott insulators. $\mathrm{Sr}_{2} \mathrm{IrO}_{4}$ with high SOC energy $\left(\lambda_{S O}=0.5 \mathrm{eV}\right.$ of Ir) is an insulator but $\mathrm{Sr}_{2} \mathrm{RhO}_{4}$ with lower SOC energy ( $\lambda_{S O}=0.15 \mathrm{eV}$ of $\left.\mathrm{Rh}\right)$ is a paramagnetic metal $[10,91]$. Hence substituting $\mathrm{Ir}$ by $\mathrm{Rh}$ in $\mathrm{A}_{2} \mathrm{IrO}_{3}$ or synthesizing pure $\mathrm{A}_{2} \mathrm{RhO}_{3}$ was our next goal. It was found from earlier chemist's report that $\mathrm{Li}_{2} \mathrm{RhO}_{3}$ is iso-structural to $\mathrm{Li}_{2} \mathrm{IrO}_{3}$ but magnetism and low temperature electrical property were not well investigated in that report [92]. In $\mathrm{Li}_{2} \mathrm{RhO}_{3}$ alternating $\mathrm{LiRh}_{2} \mathrm{O}_{6}$ and $\mathrm{Li}$ layers are stacked along crystallographic $c$-axis. In the $\mathrm{LiRh}_{2} \mathrm{O}_{6}$ layer, $\mathrm{RhO}_{6}$ edge sharing octahedra are forming the honeycomb lattice having one $\mathrm{Li}$ at the center of the honeycomb. This Rh-honeycomb lies in the crystallographic ab-plane.

There is no detail report on $\mathrm{Na}_{2} \mathrm{RhO}_{3}$, so polycrystal growth of this system was tried but all the attempts ended up with $\mathrm{NaRhO}_{2}$ (suggested by XRD). So it was concluded that $\mathrm{Na}_{2} \mathrm{RhO}_{3}$ can not be synthesized, though some percentage of Rhodium can be doped in $\mathrm{Na}_{2} \mathrm{IrO}_{3}$. Rhodium can be doped in $\mathrm{Li}_{2} \mathrm{IrO}_{3}$ also, but a significant amount of structural disorder was observed in Rh-doped $\mathrm{Li}_{2} \mathrm{IrO}_{3}$, so proper study of its magnetism was not possible. Hence in this chapter, a detail experimental and theoretical study on honeycomb lattice rhodate $\mathrm{Li}_{2} \mathrm{RhO}_{3}$ is discussed. A very similar study on $\mathrm{Li}_{2} \mathrm{RhO}_{3}$ was also published concurrently [93] but our synthesis method and study are very different and independent from them. 


\subsection{Sample synthesis}

The most energetically favored valence state of $\mathrm{Rh}$ is $3+$, but in $\mathrm{Li}_{2} \mathrm{RhO}_{3}, \mathrm{Rh}$ should be in $4+$ valence state i.e. more oxygen in the one chemical formula unit. To put enough required oxygen in the structure, this synthesis was done in a horizontal oxygen flow furnace. $\mathrm{Li}_{2} \mathrm{CO}_{3}$ and $\mathrm{Rh}$ metal powder were mixed in 1.05:1 ratio and reacted in oxygen flow at $700^{\circ} \mathrm{C}$ and $750^{\circ} \mathrm{C}$. Then it was pelletized and reacted at $800^{\circ}$ and $850^{\circ} \mathrm{C}$ for 24 and 48 hours respectively after repetitive grinding before each step. Usually for $\mathrm{Li}_{2} \mathrm{IrO}_{3}$ structurally well ordered phase forms at much higher temperature but it was found that in case of $\mathrm{Li}_{2} \mathrm{RhO}_{3}$ at higher temperatures the phase starts to structurally disorder and at $970{ }^{\circ} \mathrm{C}$ it was completely disordered. This is further discussed in the next section.

\subsection{Structural characterization}

Powder XRD (PXRD) pattern pattern of the best ordered $\mathrm{Li}_{2} \mathrm{RhO}_{3}$ sample (sintered till $850^{\circ} \mathrm{C}$ ) is shown in Fig. 7.1(a). In the figure cross marked (x) data points are experimentally obtained and red line is the theoretical fitting by Rietveld method and the blue line is the difference between them.[46, 47] All the peaks in the PXRD pattern is indexed by $\mathrm{C} 2 / \mathrm{m}$ crystal structure, same like $\mathrm{Na}_{2} \mathrm{IrO}_{3}$ and $\mathrm{Li}_{2} \mathrm{IrO}_{3}$. Detail atomic positions, lattice parameters and fitting quality parameters $\left(\mathrm{R}_{p}, \mathrm{R}_{w p}\right)$ are listed in table 7.1.
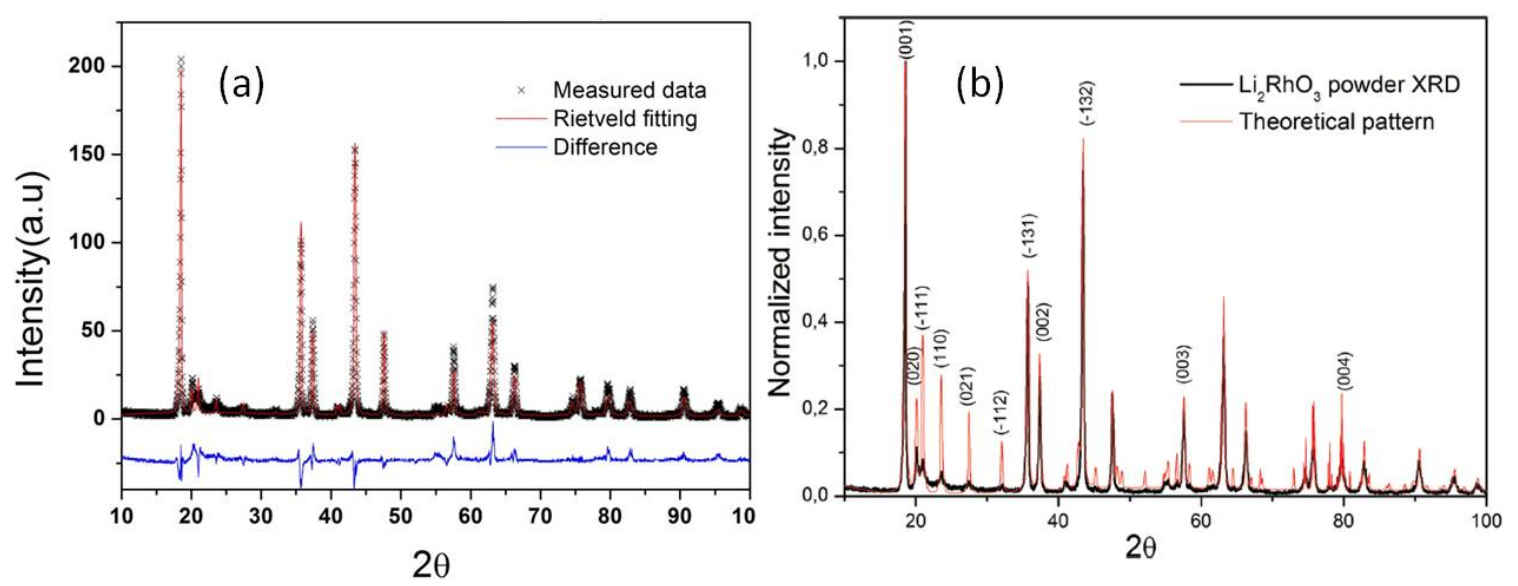

Figure 7.1: (a) Rietveld refinement of XRD data of polycrystalline $\mathrm{Li}_{2} \mathrm{RhO}_{3}$ synthesized at $850^{\circ} \mathrm{C}$ (symbols are explained in the graph).(b) Comparison of experimentally obtained XRD data and theoretical XRD data obtained from optimized crystal structure of $\mathrm{Li}_{2} \mathrm{RhO}_{3}$, reprinted from [94] 
Table 7.1: Structural parameters of $\mathrm{Li}_{2} \mathrm{RhO}_{3}$ obtained fr Rietveld refinements of powder XRD pattern. Lattice parameters are: $(a=5.1227(6) \AA, b=8.8389$ (om 10$) \AA, c=$ $\left.5.0979(5) \AA, \beta=109.660(10)^{\circ}\right)$ and the space group is $C 2 / m$. Fitting quality: $\mathrm{Chi}^{2}$ $=0.4320, \mathrm{R}_{w p}=0.253, \mathrm{R}_{p}=0.202$.

\begin{tabular}{ll|ccccc}
\hline atom & position & $\mathrm{x}$ & $\mathrm{y}$ & $\mathrm{z}$ & Occ. & $\mathrm{B}$ \\
\hline \hline $\mathrm{Rh}$ & $4 \mathrm{~h}$ & 0 & $0.3307(4)$ & 0 & 0.7 & 0.01 \\
$\mathrm{Li}$ & & 0 & $0.3305(4)$ & 0 & 0.3 & 0.02 \\
\hline $\mathrm{Li}$ & $2 \mathrm{a}$ & 0 & 0 & 0 & $0.613(4)$ & 0.02 \\
$\mathrm{Rh}$ & & 0 & 0 & 0 & $0.387(4)$ & 0.01 \\
\hline $\mathrm{Li}$ & $4 \mathrm{~g}$ & 0 & $0.454(34)$ & 0.5 & 1 & 0.02 \\
$\mathrm{Li}$ & $2 \mathrm{c}$ & 0 & 0.5 & 0.5 & 1 & 0.02 \\
$\mathrm{O}$ & $8 \mathrm{j}$ & $0.2678(32)$ & $0.2877(17)$ & $0.7300(18)$ & 1 & 0.03 \\
$\mathrm{O}$ & $4 \mathrm{i}$ & $0.272(4)$ & 0 & $0.7440(32)$ & 1 & 0.03 \\
\hline
\end{tabular}

In the Fig. 7.1(a) peaks between $2 \theta=19$ to $33^{\circ}$ has smaller relative intensity compared to $\mathrm{Li}_{2} \mathrm{IrO}_{3}$ which is due to stacking fault of $\mathrm{LiRh}_{2} \mathrm{O}_{6}$ layers. In the Rietveld fitting, this stacking fault is taken into account by site mixing of $\mathrm{Rh}$ and $\mathrm{Li}$ position, $4 \mathrm{~h}$ and $2 \mathrm{a}$ respectively in the $\mathrm{LiRh}_{2} \mathrm{O}_{6}$ layer (Table 7.1). To get a reasonable fitting quality, 30\% site mixing between $\mathrm{Rh}$ and $\mathrm{Li}$ is needed which is higher than the $\mathrm{Li}_{2} \mathrm{IrO}_{3}$ case, indicates more stacking fault in Rh-system. But it is already discussed in the chapter 4, the Rietveld analysis is not the best way to treat the stacking fault and one should not consider the site mixing used here as true site exchange, the achieved true $S_{\text {eff }}=1 / 2$ fluctuating moment does not support that. The lattice parameters obtained are $a=5.1227(6) \AA, b=8.8389(10) \AA, c=5.0979(5) \AA$, $\beta=109.660(10)^{\circ}$ and cell volume $217.373(31) \AA^{3}$. c/a ratio is 0.995 , do not differ significantly compared to $\mathrm{Li}_{2} \mathrm{IrO}_{3}$. Rh-Rh distances obtained are 2.997 and $2.930 \AA$, Rh-O distances varies from 2.070 to $2.393 \AA$. This suggests that $\mathrm{RhO}_{6}$ octahedra obtained from the Rietveld fitting is very much distorted. This is always observed in the Rietveld refinement of PXRD pattern of all layered $\mathrm{A}_{2} \mathrm{TO}_{3}(\mathrm{~A}=\mathrm{Na}, \mathrm{Li} ; \mathrm{T}=\mathrm{It}, \mathrm{Rh}, \mathrm{Ru}$, Pt etc.) $[52,56]$. Main reasons are: firstly, lab PXRD does not have good enough resolution which can resolve the intensities due to oxygen and secondly, the Rietveld refinement can not take into account the stacking fault very well. So this result give a little unstable structure. Band structure calculation with such a unstable structure is not desirable, so our collaborators Mazin et. al. did the band structure calculation using optimized atomic structure obtained from VASP, using experimental lattice parameters [94].

Optimized atomic positions are listed in table 7.2. Rh-Rh distances are 2.951 and $2.952 \AA$ and Rh-O bond lengths are 2.023, 2.030 and $2.021 \AA$. This gives a very ideal octahedra. But theoretically simulated XRD pattern from this optimized structure does not differ much with the experimentally obtained PXRD [see Fig. 7.1(b)]. Most significant 
Table 7.2: Optimized crystal structure of $\mathrm{Li}_{2} \mathrm{RhO}_{3}$, using experimental lattice parameters and space group $C 2 / m$. Reprinted from [94].

\begin{tabular}{l|l|l|l|l}
\hline atom & position & $x$ & $y$ & $z$ \\
\hline $\mathrm{Rh}$ & $4 \mathrm{~h}$ & 0 & 0.333 & $1 / 2$ \\
$\mathrm{Li}$ & $2 \mathrm{a}$ & 0 & 0 & 0 \\
$\mathrm{Li}$ & $4 \mathrm{~g}$ & 0 & 0.660 & 0 \\
$\mathrm{Li}$ & $2 \mathrm{c}$ & 0 & 0 & $1 / 2$ \\
$\mathrm{O}$ & $8 \mathrm{j}$ & 0.516 & 0.327 & 0.263 \\
$\mathrm{O}$ & $4 \mathrm{i}$ & 0.002 & $1 / 2$ & 0.7380 \\
\hline
\end{tabular}

difference are observed in the $2 \theta$-region 19 to $33^{\circ}$, which is attributed to stacking fault in the real material and discrepancy to determine by the Rietveld analysis. But to experimentally determine $\mathrm{Li}_{2} \mathrm{RhO}_{3}$ atomic parameters very accurately pair distribution function (PDF) analysis can be done with total scattering obtained from synchrotron facility. Same analysis has been done on $\mathrm{Li}_{2} \mathrm{IrO}_{3}$ [58]. $850^{\circ} \mathrm{C}$ sintered $\mathrm{Li}_{2} \mathrm{RhO}_{3}$ has some amount of stacking fault but the samples sintered at higher temperature are more disordered.

\subsubsection{Disorder in $\mathrm{Li}_{2} \mathrm{RhO}_{3}$}

For $\mathrm{Li}_{2} \mathrm{IrO}_{3}$ sintering at higher temperature or a $\mathrm{Li}$ enriched environment ( $\mathrm{LiCl}$ flux) is required to obtain structurally ordered phase so same is done for $\mathrm{Li}_{2} \mathrm{RhO}_{3}$. After pelletizing $\mathrm{Li}_{2} \mathrm{RhO}_{3}$ is sintered at higher temperature till $970^{\circ}$ in $\mathrm{O}_{2}$ flow. With the increasing temperature at $900^{\circ} \mathrm{C}$ and $950^{\circ} \mathrm{C}$, the PXRD peaks in between $2 \theta=19$ to $30^{\circ}$ is decreasing in height having a continuous slop between the peaks which is attributed to Warren fall [Fig. 7.2 (a)and (b)]. This is typical signature of structural fault in the system as discussed earlier. At $970^{\circ}$ all the peaks in that region vanished [Fig. 7.2 (a) and (b)] and XRD pattern was fitted with a different structure, a cubic crystal system with $\mathrm{F}$ d $\overline{3} \mathrm{~m}$ space group (space group number 227). The phase is identified by Rietveld refinement as $\beta-\mathrm{LiRhO}_{2}$ (Appendix B). This suggests that at higher temperature $\mathrm{Rh}$ goes from +4 valence state to +3 valence state, hence $\mathrm{Li}_{2} \mathrm{RhO}_{3}$ transforms into $\mathrm{LiRhO}_{2}$.

Another type of disordered phase is observed when $\mathrm{Li}_{2} \mathrm{RhO}_{3}$ was sintered in $\mathrm{LiCl}$. All the peaks between $2 \theta=19$ to $30^{\circ}$ in the PXRD are absent. LiCl environments creates oxygen deficient environment which results another polymorph of $\mathrm{LiRhO}_{2}$. Basically peaks in this region represents coherent ordering of honeycomb layer which is absent in 3D structure of $\mathrm{LiRhO}_{2}$. PXRD pattern of $\mathrm{LiCl}$ sintered $\mathrm{Li}_{2} \mathrm{RhO}_{3}$ (SM12S) was fitted with Trigonal $\mathrm{R} \overline{3} \mathrm{~m}$ 

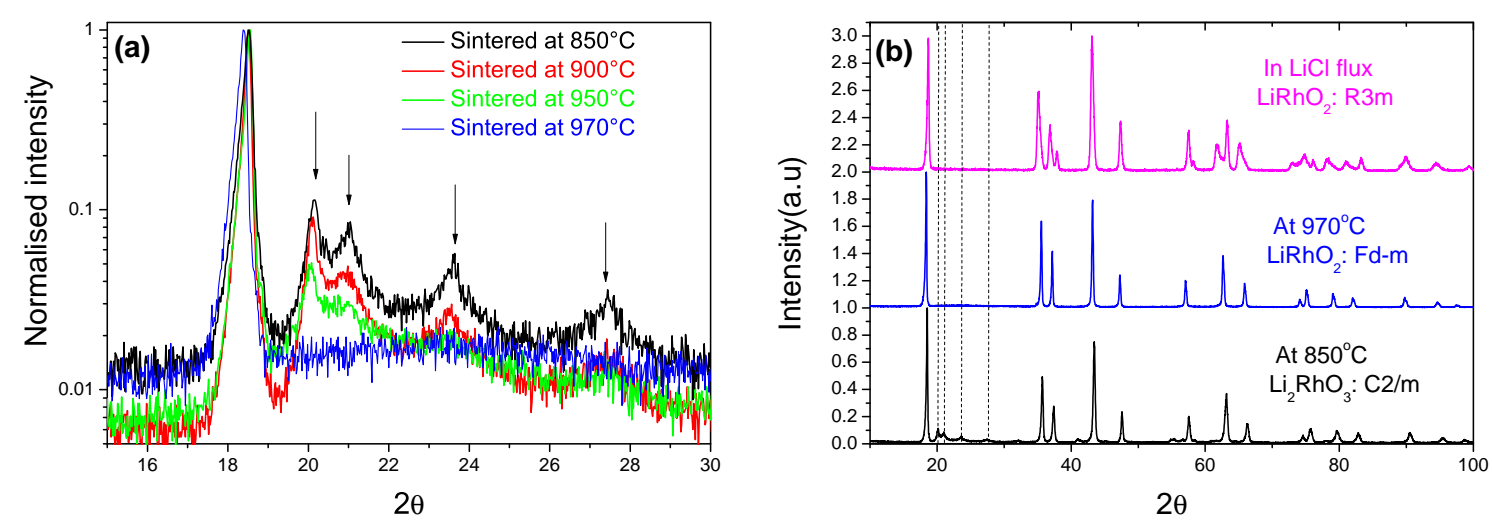

Figure 7.2: (a) Powder $\mathrm{XRD}$ of $850^{\circ} \mathrm{C}, 900^{\circ} \mathrm{C}, 950^{\circ} \mathrm{C}$ and $970^{\circ} \mathrm{C}$ sintered $\mathrm{Li}_{2} \mathrm{RhO}_{3}$ sample in between $2 \theta=19$ to $30^{\circ}$. (b) Powder XRD of ordered $850^{\circ} \mathrm{C}$, disordered $970^{\circ} \mathrm{C}$ sintered $\mathrm{Li}_{2} \mathrm{RhO}_{3}$ and $\mathrm{Li}_{2} \mathrm{RhO}_{3}$ prepared in $\mathrm{LiCl}$ flux.

: h crystal structure (space group no. 166). Rietveld fitting and atomic parameters are in Appendix B.

If a disordered $\mathrm{Li}_{2} \mathrm{RhO}_{3}$ phase is annealed in $\mathrm{O}_{2}$ pressure at $800^{\circ}$ for $24-48$ hours, the PXRD peak heights in between $2 \theta=19$ to $30^{\circ}$ increases in height and same effect was observed when $\mathrm{Li}_{2} \mathrm{RhO}_{3}$ was annealed in ozone $\left(\mathrm{O}_{3}\right)$ furnace at $400^{\circ} \mathrm{C}$ for 6 hours. This indicates that by reducing oxygen deficiency in the phase one can reduce structural disorder in the $\mathrm{Li}_{2} \mathrm{RhO}_{3}$ system.

Hence we conclude that $850^{\circ} \mathrm{C} \mathrm{O}_{2}$ sintered $\mathrm{Li}_{2} \mathrm{RhO}_{3}$ is the best structurally ordered sample and all the bulk and local probes are done on this sample.

\subsection{Electrical resistivity}

$\mathrm{Li}_{2} \mathrm{IrO}_{3}$ is an AF insulator due to strong SOC. Now in $\mathrm{Li}_{2} \mathrm{RhO}_{3} \mathrm{SOC}$ is very low $\left(\lambda_{S O} \approx\right.$ $0.1 \mathrm{eV}$ ) hence SOC alone can not open insulating gap in the $t_{2 g}$ band of $\mathrm{Li}_{2} \mathrm{RhO}_{3} 4 \mathrm{~d}^{5}$ electronic state. Rather with 5 electrons in $t_{2 g}$ (partially filled) $\mathrm{Li}_{2} \mathrm{RhO}_{3}$ was expected to be a metal same like $\mathrm{Rh}$ analogue of $\mathrm{Sr}_{2} \mathrm{IrO}_{4}: \mathrm{Sr}_{2} \mathrm{RhO}_{4}$ [91]. High temperature study on $\mathrm{Li}_{2} \mathrm{RhO}_{3}$ previously reported it as stable semiconductor [92].

Resistivity of the well ordered $\mathrm{Li}_{2} \mathrm{RhO}_{3}$ increases with decreasing temperature (Fig. 7.3). Resistivity was measured on several piece of highly pressurized pellets, finally sintered at $850^{\circ} \mathrm{C}$. Order of magnitude of room temperature resistivity were same at every measurement. 

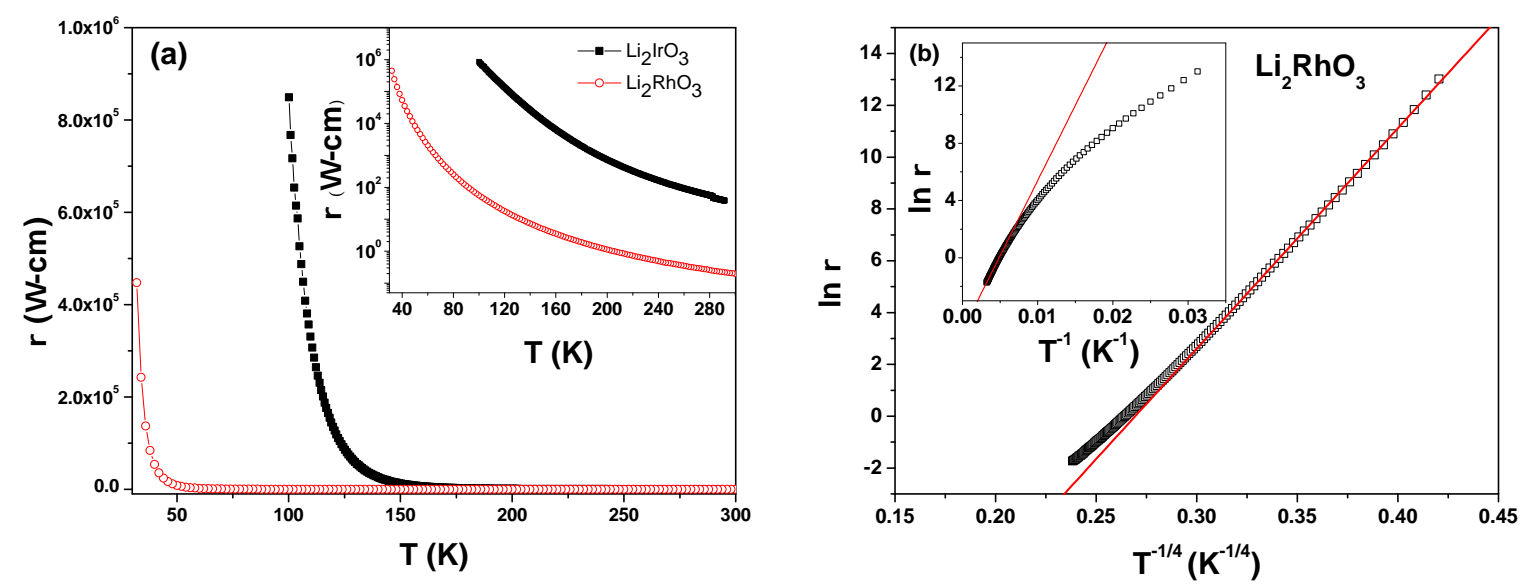

Figure 7.3: (a) Comparison of electrical resistivity $\rho(T)$ between $\mathrm{Li}_{2} \mathrm{RhO}_{3}$ and $\mathrm{Li}_{2} \mathrm{IrO}_{3}$, inset shows the plot in logarithmic scale. (b) Fitting of $\mathrm{Li}_{2} \mathrm{RhO}_{3} \ln \rho(T)$ versus $(1 / T)^{1 / 4}$ by Mott variable range hopping behavior, inset shows $\ln \rho(T)$ versus $1 / T$ data fitted at high $T$ by Arrhenius behavior to obtain activation gap.

Hence one can conclude this resistive behavior is not due to grain boundaries. Also study by Y.Luo et.al reported resistive behavior of $\mathrm{Li}_{2} \mathrm{RhO}_{3}$ [93].

Comparative study of $\mathrm{Li}_{2} \mathrm{IrO}_{3}$ and $\mathrm{Li}_{2} \mathrm{RhO}_{3}$ resistivity suggests that $\mathrm{Li}_{2} \mathrm{RhO}_{3}$ is less resistive than $\mathrm{Li}_{2} \mathrm{IrO}_{3}$ (Fig. 7.3a), resistivity of $\mathrm{Li}_{2} \mathrm{RhO}_{3}$ is almost two order of magnitude smaller than its Ir counterpart (inset). Contrary to semiconducting report by Todorva et. al. it was found that $\mathrm{Li}_{2} \mathrm{RhO}_{3}$ resistivity does not follow Arrhenius activated behavior (Eqn. 4.3), it deviates significantly at lower temperature $(T)$ (inset, Fig. 7.3 b). Hence resistivity of $\mathrm{Li}_{2} \mathrm{RhO}_{3}$ is not semiconductor type, rather very sharp increase of $\rho$ versus $T$ similar to $\mathrm{Li}_{2} \mathrm{IrO}_{3}$ indicates a correlated or relativistic insulating behavior. Arrhenius fitting of $\ln \rho$ versus $1 / \mathrm{T}$ data at high temperature (between 200-300K) gives activation gap equal to $85 \mathrm{meV}$ compared to $159 \mathrm{meV}$ for $\mathrm{Li}_{2} \mathrm{IrO}_{3}$. Same kind of activation gap is reported by Y.Luo et.al. and in previous reports also [92, 93].

$\mathrm{Li}_{2} \mathrm{RhO}_{3}$ resistivity fits more to $3 \mathrm{D}$ variable range hopping (VHR) behavior (Eqn. 4.5) same like $\mathrm{A}_{2} \mathrm{IrO}_{3} . \ln \rho$ versus $(1 / \mathrm{T})^{1 / 4}$ follows linear behavior slightly deviating at higher temperature (Fig. 7.3b). Fitting gives $T_{\circ} \approx 10^{7} \mathrm{~K}$ and $\rho_{\circ} \approx 10^{-4} \Omega$-cm. Similarity in the temperature dependence of the $\mathrm{Li}_{2} \mathrm{RhO}_{3}$ and $\mathrm{Li}_{2} \mathrm{IrO}_{3}$ resistivity clearly indicates that their electronic structure may not be much different. Later, the real origin of this unexpected resistive behavior is explained by DFT calculation. 


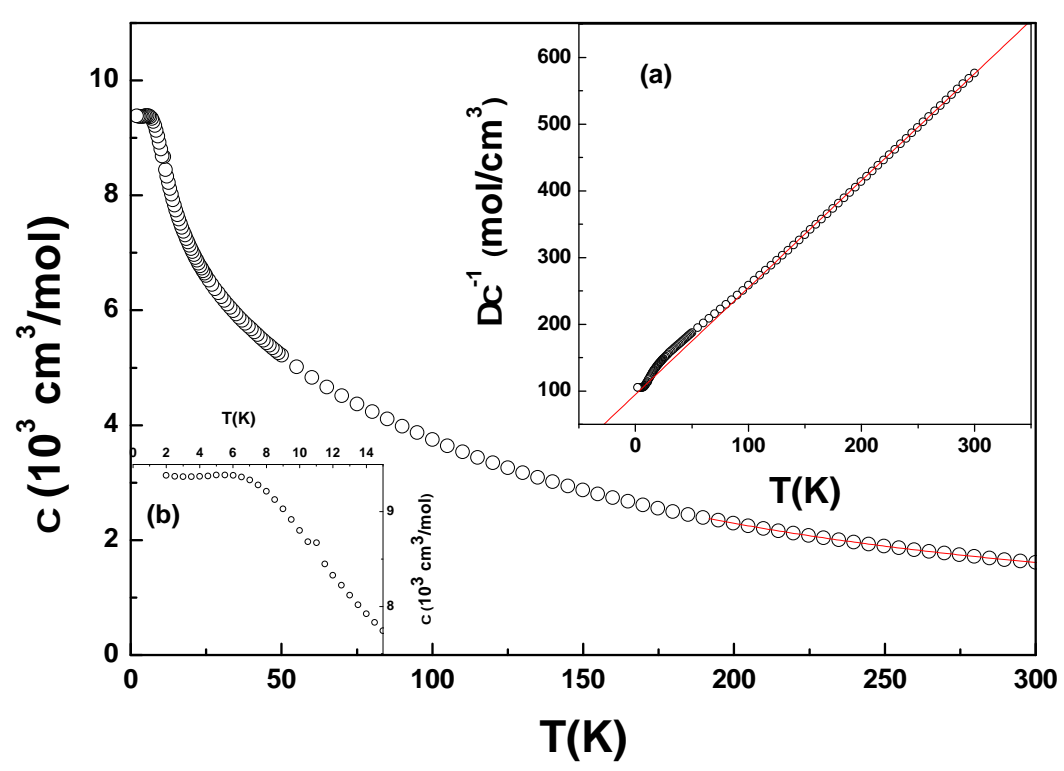

Figure 7.4: Magnetic molar susceptibility $(\chi)$ of $\mathrm{Li}_{2} \mathrm{RhO}_{3}$. Red line is the $\mathrm{CW}$ fitting. Inset(a) $\Delta \chi\left(=\chi-\chi_{0}\right)$ has been plotted which is fitted with CW law (red straight line) for value of $\Theta_{W}\left(\chi_{0}\right.$ was obtained from CW fitting of $\chi$ vs T data). Inset(b) Low temperature kink in $\chi$ at $6 \mathrm{~K}$.

\subsection{Magnetic property}

Magnetization(M) of well ordered $\mathrm{Li}_{2} \mathrm{RhO}_{3}$ was measured on different batches of the sample under magnetic field $(H)$ of $1 \mathrm{~T}$. From this magnetic molar susceptibility $(\chi(T))=M / H)$ is calculated. Susceptibility follows Curie-Weiss (CW) behavior (Eqn 4.7). CW fitting of $\chi(T)$ versus $T$ data in the temperature range of 200 to $300 \mathrm{~K}$ gives $\chi_{0}=-1.235(18) \times 10^{-4}$ $\mathrm{cm}^{3} /$ mol. Then $1 / \Delta \chi$ versus $T$ data is fitted with $C W$ law (inset, Fig. 7.4), where $\Delta \chi=$ $\chi-\chi_{0}$. From the fitting obtained $\mathrm{CW}$ temperature $\Theta_{W}=-59.5(1) \mathrm{K}$ which confirms strong AF interaction between fluctuating $S=1 / 2$ moments of Rh4+. But effective moment obtained is $2.24 \mu_{B}$ which is little higher than effective moment for $\mathrm{S}=1 / 2,1.73 \mu_{B}$. This indicates that some orbital angular moment of $\mathrm{Rh} t_{2 g}$ orbitals are not quenched and that unquenched orbital angular moment $\left(\mathrm{L}_{e f f}=1\right)$ contributes to the effective magnetic moment. Unquenched orbital angular moment of a TM ion with octahedral crystal field can arise for degenerate ground sates of that TM ion.[19] Now one has to find the possibility and source of this degeneracy.

Inset (b) of the Fig. 7.4 shows a low temperature kink in $\chi(T)$. To understand the origin such kink in $\chi(T)$ FC-ZFC susceptibility was measured at low field $\mathrm{H}=0.005 \mathrm{~T}$. A clear hysteresis is observed. ZFC susceptibility shows a clear drop after $6 \mathrm{~K}$ and FC 


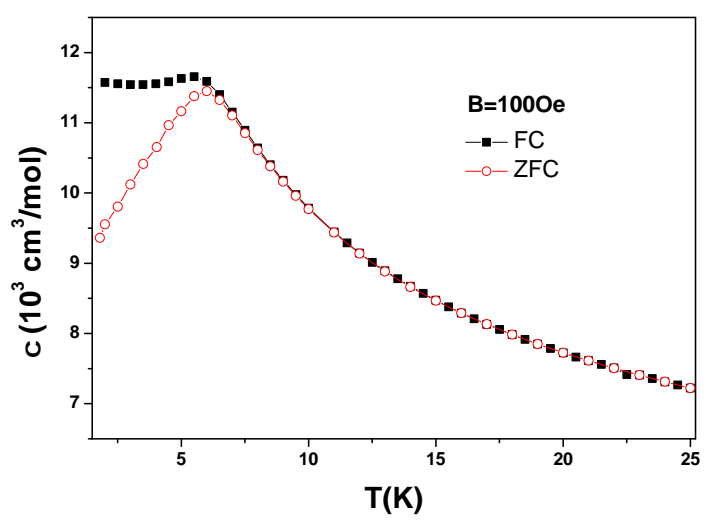

Figure 7.5: FC and ZFC susceptibility of $\mathrm{Li}_{2} \mathrm{RhO}_{3}$ measured in $\mathrm{H}=0.005 \mathrm{~T}$, clear separation from $6 \mathrm{~K}$ confirms spin glass freezing below $6 \mathrm{~K}$.

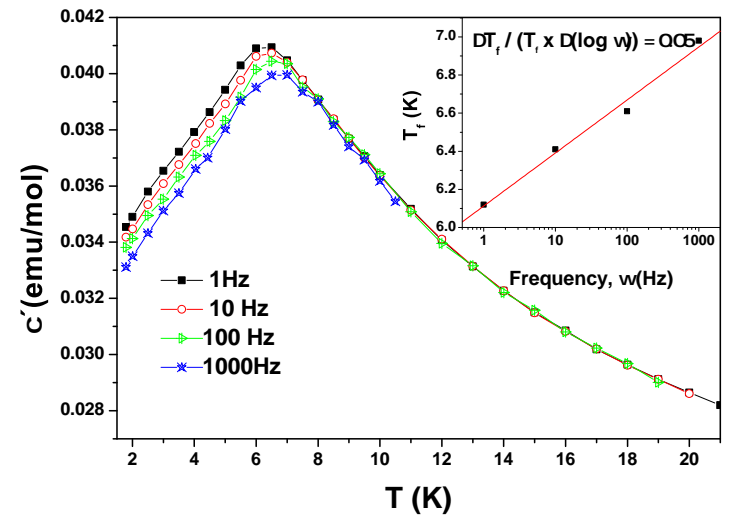

Figure 7.6: AC susceptibility of $\mathrm{Li}_{2} \mathrm{RhO}_{3}$ measured at different frequencies. Frequency dependence of spin-glass temperature $\left(\mathrm{T}_{g}\right)$ is plotted in the inset.

susceptibility is clearly separated from ZFC susceptibility. This separation between FC and ZFC susceptibility is characteristic signature of spin-glass (SG) freezing same observed in $\mathrm{Na}_{2}\left(\mathrm{Ir}_{1-x} \mathrm{Ti}_{x}\right) \mathrm{O}_{3}$ and $\mathrm{Li}_{2}\left(\operatorname{Ir}_{1-x} \mathrm{Ti}_{x}\right) \mathrm{O}_{3}$ and $T_{g}=6 \mathrm{~K}$ is the SG freezing temperature. Ac susceptibility $\left(\chi^{\prime}\right)$ was measured with lowest possible driving field $(=0.0004 \mathrm{~T})$ at different frequencies. A clear cusp is seen at $T_{g}$ which shifts towards lower temperature with increasing frequency $(\omega)$. The position of cusp in $\chi^{\prime}$ at different $\omega$ is identified as $\mathrm{T}_{f}$ (Fig. 7.6). $\mathrm{T}_{f}$ versus $\omega$ is plotted in inset in $\omega$ logarithmic scale. From the slope of the curve frequency dependence per decade is quantified by the term $\Delta T_{f} /\left(T_{g} \Delta \log \omega\right)$, in case of $\mathrm{Li}_{2} \mathrm{RhO}_{3}$ it is 0.05 . This is quite weak frequency dependence compared to a local moment insulating system (as it was 0.11 for $\mathrm{Na}_{2}\left(\operatorname{Ir}_{1-x} \mathrm{Ti}_{x}\right) \mathrm{O}_{3}$ ). Still it is two times higher than canonical spin glasses. [95] Resistivity decreases quite a lot in $\mathrm{Li}_{2} \mathrm{RhO}_{3}$ compared to $\mathrm{Li}_{2} \mathrm{IrO}_{3}$, this may be the cause of weaker frequency dependence in $\mathrm{Li}_{2} \mathrm{RhO}_{3}$.

Hence magnetic property clearly hint at the presence of a degenerate ground state. In a degenerate ground state spins are frustrated either by geometrically frustration or by dynamical frustration of different exchange interactions. In such frustrated system a little disorder can force the spins to freeze at lower temperature which may be the cause of SG behavior in $\mathrm{Li}_{2} \mathrm{RhO}_{3}$. 


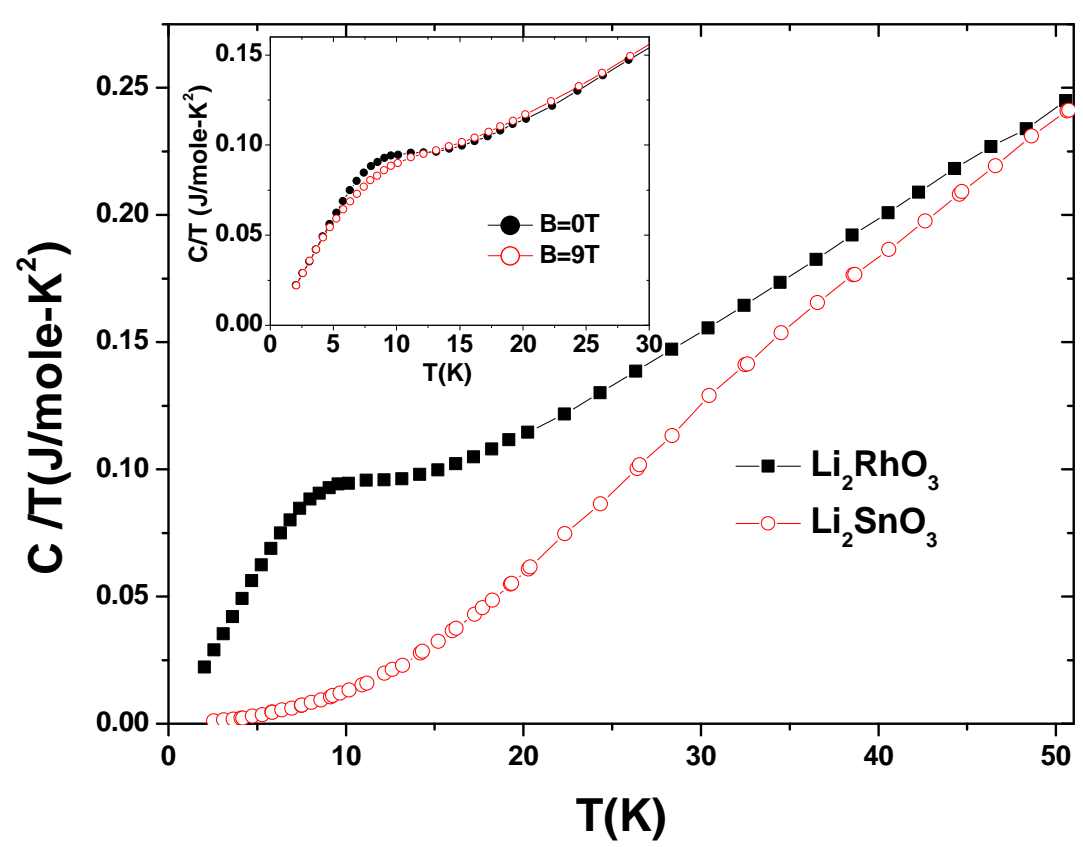

Figure 7.7: Heat capacity divided by temperature $(\mathrm{C} / \mathrm{T})$ versus temperature $(\mathrm{T})$ for $\mathrm{Li}_{2} \mathrm{RhO}_{3}$ and lattice contribution, measured in $\mathrm{Li}_{2} \mathrm{SnO}_{3}$. In the inset $\mathrm{C} / \mathrm{T}$ versus $\mathrm{T}$ data of $\mathrm{Li}_{2} \mathrm{RhO}_{3}$ measured in magnetic field $\mathrm{B}=0 \mathrm{~T}$ and $9 \mathrm{~T}$.

\subsection{Heat capacity}

Compared to all other measurements on a spin-glass, heat capacity is least informative. Fig. 7.7 shows heat capacity divided by temperature $C / T$ of $\mathrm{Li}_{2} \mathrm{RhO}_{3}$ and $\mathrm{Li}_{2} \mathrm{SnO}_{3}$, contribution of lattice heat capacity for $\mathrm{Li}_{2} \mathrm{RhO}_{3} . \mathrm{Li}_{2} \mathrm{RhO}_{3} C / T$ shows a broad maximum above $T_{g}\left(\mathrm{~T}_{\max }=10 \mathrm{~K}\right)$, no peak like feature or any singularity is observed. This broad maximum becomes even less pronounced in magnetic field. At 9T magnetic field $C / T$ maximum is much broadened (inset, Fig. 7.7).

Sometimes magnetic contribution of the heat capacity contains more information than the total heat capacity. Magnetic contribution of the heat capacity $(\Delta C)$ is calculated by subtracting lattice heat capacity from total heat capacity of $\mathrm{Li}_{2} \mathrm{RhO}_{3}$. Fig. 7.8 shows $\Delta C$ versus $T$ data, a broad feature over the whole temperature range of $2-50 \mathrm{~K}$ is observed. Value of $\Delta \mathrm{C}$ increase till $15 \mathrm{~K}$ then slowly decreases till $50 \mathrm{~K} . \Delta C / T$ rather show a sharper feature. It shows a broad maximum around $10 \mathrm{~K}\left(T_{\max }=9 \mathrm{~K}\right)$, with the magnetic field of $9 \mathrm{~T}$ this broad maximum becomes little broader and shifts to higher temperature. This indicates shift of magnetic entropy at higher temperature due to shift of more and more frozen spin degrees of freedom to higher temperature by the application of magnetic field. Zero-field entropy $(\Delta S)$ is calculated by integrating $\Delta C / T$ versus $T$ data for zero field. $\Delta S$ versus 


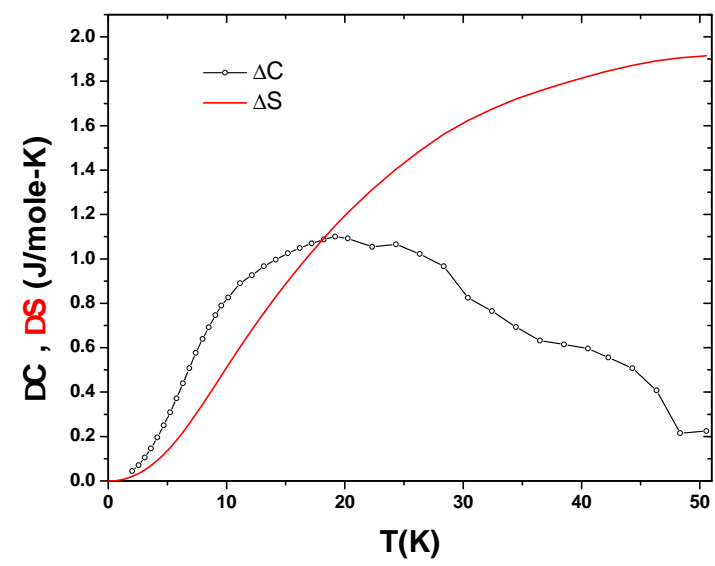

Figure 7.8: Magnetic heat capacity $(\Delta \mathrm{C})$ versus temperature $(T)$ for $\mathrm{Li}_{2} \mathrm{RhO}_{3}$ plotted in open symbol. Magnetic entropy $(\Delta \mathrm{S})$ calculated integrating $\Delta C / T$ versus $\mathrm{T}$ data, plotted in red line.

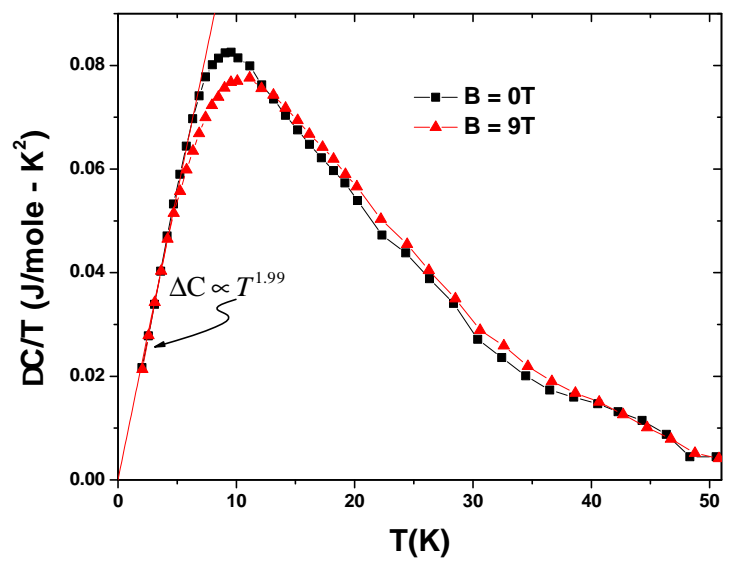

Figure 7.9: Magnetic heat capacity divided by temperature $(\Delta \mathrm{C} / \mathrm{T})$ versus $\mathrm{T}$ data for $\mathrm{Li}_{2} \mathrm{RhO}_{3}$, at $B=0 \mathrm{~T}$ and $9 \mathrm{~T}$. Red lines shows a fitting of the $\Delta \mathrm{C} / \mathrm{T}$ vs $\mathrm{T}$ data below $T_{g}$.

$T$ is plotted in Fig. 7.8 (Red line). At $T_{g}, \Delta S=0.2 \mathrm{~J} /$ mole-K which is only $5 \%$ of $R \ln 2$. All the remaining entropy is frozen out far above $T_{g}$. Upto $50 \mathrm{~K}$ only $25 \%$ total magnetic entropy is recovered.

In the frustrated $\mathrm{A}_{2} \mathrm{IrO}_{3}$, entropy at $T_{N}=15-20 \% \mathrm{R} \ln 2$ only. Usually for frustrated systems magnetic entropy is distributed over the temperature scale equivalent to $\Theta_{w} \cdot \mathrm{Li}_{2} \mathrm{RhO}_{3}$ is a magnetically frustrated system having $\Theta_{w}=-53 \mathrm{~K}$ hence its magnetic entropy is distributed over this temperature range added with frozen entropy due to spinglass state. Hence very small magnetic entropy is obtained at $T_{g}$ in $\mathrm{Li}_{2} \mathrm{RhO}_{3}$. Also one has to remember this entropy calculation is highly dependent upon accurate subtraction of lattice contribution. $\mathrm{Li}_{2} \mathrm{SnO}_{3}$ is considered as lattice contribution in case of both $\mathrm{Li}_{2} \mathrm{IrO}_{3}$ and $\mathrm{Li}_{2} \mathrm{RhO}_{3}$ which can not be very accurate for both the system because atomic mass of $\mathrm{Rh}$, Ir and Sn differs significantly, in spite of very similar ionic radius of their $4+$ ionic state.

Magnetic heat capacity $\Delta C / T$ curve can be fitted with a power law behavior: $\Delta C / T=$ $A T^{\alpha}$ below $T_{g}$ for both $\mathrm{B}=0 \mathrm{~T}$ data and $9 \mathrm{~T}$ data. From the fitting $\alpha=0.99(2)$ and $A=0.011(1)$. Hence magnetic contribution of the heat capacity $\Delta C$ basically follow $T^{2}$ behavior; $\Delta \mathrm{C} \propto \mathrm{T}^{1.99}$. Such $T^{2}$-behavior were seen for spin-singlet state or spin-liquid state where gapless excitations are generated from collective modes of short-ranged spinclusters $[82,96,97]$. Usually in local-probe measurements footprints of such excitations are more evidential. 


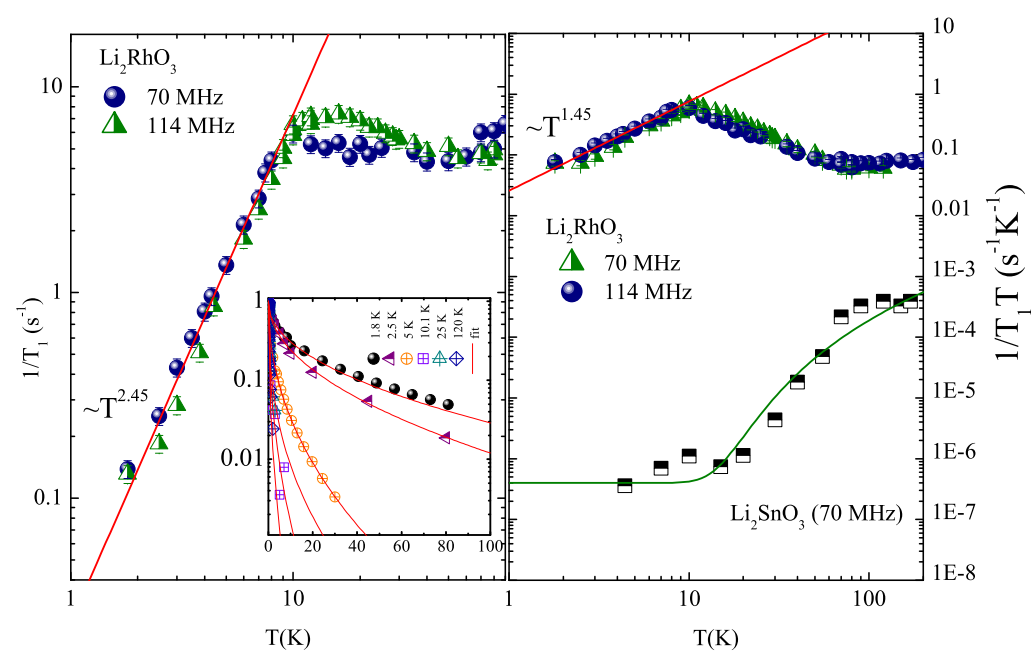

Figure 7.10: (a) Field sweep ${ }^{7}$ li NMR spectra in $\mathrm{Li}_{2} \mathrm{RhO}_{3}$ at different temperatures. (b) The temperature dependence of full width at half maxima (FWHM) in two applied magnetic fields in $\mathrm{Li}_{2} \mathrm{RhO}_{3}$ compared with that non-magnetic analog $\mathrm{Li}_{2} \mathrm{SnO}_{3}$. The inset shows the variation of FWHM with bulk susceptibility with temperature as an implicit parameter. Plots obtained from private communication with P. Khuntia.[98]

\subsection{Nuclear Magnetic Resonance(NMR)}

In order to have a microscopic insight of the observed spin-freezing and spin-dynamics of $\mathrm{Li}_{2} \mathrm{RhO}_{3}{ }^{7} \mathrm{Li} \mathrm{NMR}$ line width and spin lattice relaxation were studied by our collaborator P. Khuntia in the NMR group of MPI-CPFS, Dresdren.[98] It was done on well characterized sample, prepared in our lab.

Fig. 7.10(a) show field dependence of ${ }^{7} \mathrm{Li}$ NMR spectra measured at resonant frequency $\nu=70 \mathrm{MHz}$. A temperature independent NMR shift down to $1.8 \mathrm{~K}$ confirms absence of any long-range order and such behavior is very commonly seen in other short range ordered systems also [99]. An inhomogeneous broadening of the spectra below $T_{g}$ suggests local disorder which resulted this spin-glass behavior. The temperature dependence of the full width at half maximum (FWHM) peak intensity of the spectra is plotted in Fig. 7.10(b). The FWHM versus $T$ can be well fitted with CW behavior. It scales very well with $\chi(T)$ above $T_{g}$ (inset) and significantly deviates below. FWHM increases much faster than the magnetization with decreasing temperature because a correlation between antiferromagnetically coupled clusters set up at $T_{g}[98,99]$. But NMR peak intensity does not change much in low- $T$ region this suggests a partial freezing of spins due to stacking disorder [98]. 


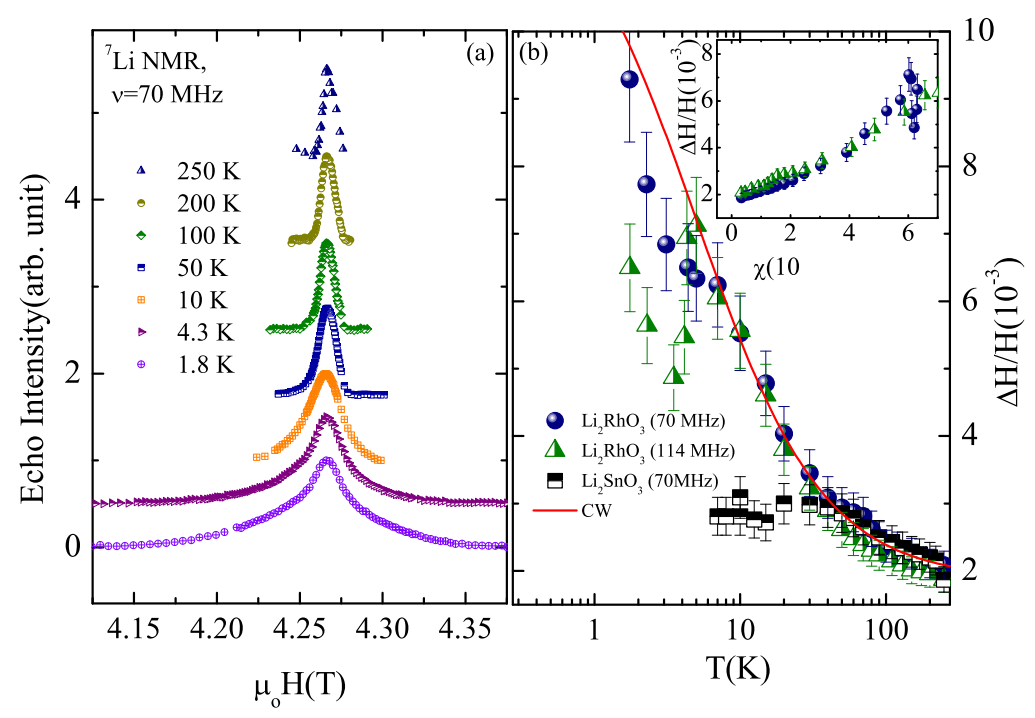

Figure 7.11: (a) The temperature dependence of $1 / T_{1}$ in two applied magnetic field and soild line is power law fitting. The inset shows the recoveries of longitudinal magnetization with stretched exponential fit at various temperatures. (b) Temperature dependence of $1 / T_{1} T$ of $\mathrm{Li}_{2} \mathrm{RhO}_{3}$ compared with $\mathrm{Li}_{2} \mathrm{SnO}_{3}$. Plots obtained from private communication with P. Khuntia.[98]

The spin lattice relaxation $\left(T_{1}\right)$ is plotted in Fig. 7.11. $T_{1}$ was calculated by fitting a stretched exponential behavior to the longitudinal nuclear magnetization behavior [inset, Fig. 7.11(a)]. Most importantly both $1 / T_{1}$ and $1 / T_{1} T$ are independent of temperature at high- $T$ and strongly enhance in the intermediate $T$-region with a peak around spin-glass transition. Below $T_{g} 1 / T_{1}$ strongly drops following a power law behavior $T^{2.45}$. The decrease of $1 / T_{1}$ following a typical power law behavior below $T_{g}$ is similar to other Ir-based spin liquid system $\mathrm{Na}_{4} \mathrm{Ir}_{3} \mathrm{O}_{8}$ and organic spin liquid systems, where degeneracy of low lying excitations added with fluctuating magnetic moment control the spin-dynamics [98]. But real picture of spin-degeneracy only can be understood after a rigorous DFT study with different possible spin correlations.

\subsection{Band structure calculation}

$\mathrm{Li}_{2} \mathrm{RhO}_{3}$ with much less spin-orbit coupling (SOC) was expected to be a metal like $\mathrm{Sr}_{2} \mathrm{RhO}_{4}$. But experiment clearly suggest that it is still insulating possibly with reduced band gap. Naive way to say, $\mathrm{Li}_{2} \mathrm{RhO}_{3}$ would be a Mott insulator driven by Coulomb repulsion $U$ but to visualize a real picture band structure calculation is needed. Also bulk measurements and 


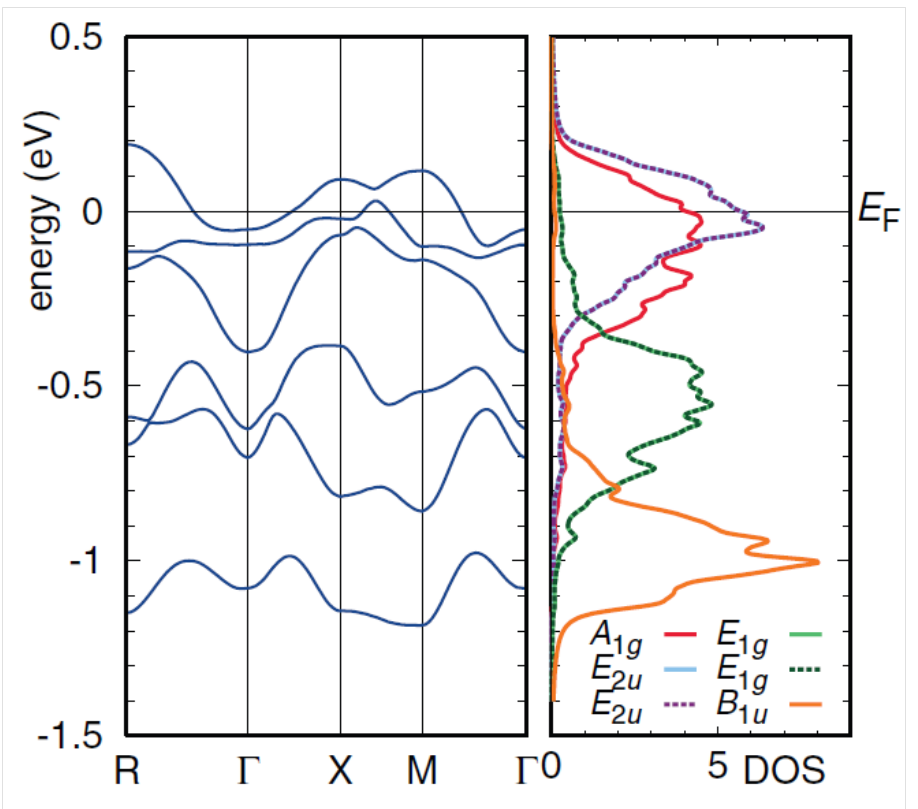

Figure 7.12: Nonrelativistic and nonmagnetic band structure and density of states of $\mathrm{Li}_{2} \mathrm{RhO}_{3}$ projected on QMO. Reprinted from [94].

local probes hinted that spin-glass freezing in $\mathrm{Li}_{2} \mathrm{RhO}_{3}$ may have originated from degenerate magnetic ground state. For true picture of the possible magnetic ground state a spinpolarized calculation is needed. Our collaborators Mazin et. al. have done band structure calculation projecting $\mathrm{Li}_{2} \mathrm{RhO}_{3}$ on quasi molecular orbitals (QMOs), using our experimental lattice parameters and their optimized atomic parameters listed in Table 7.2 [94].

With the optimized atomic parameters, first principle calculations were performed which resulted all the tight-binding parameters. Band structure calculation with those parameters projecting on QMO picture shown in Fig. 7.12. Compared to $\mathrm{Na}_{2} \mathrm{IrO}_{3}$, overlapping QMOs are observed in $\mathrm{Li}_{2} \mathrm{RhO}_{3}$ rather than isolated manifolds [40]. A huge DOS of state at $\mathrm{E}_{F}$ clearly project $\mathrm{Li}_{2} \mathrm{RhO}_{3}$ as a metal in nonrelativistic and nonmagnetic band structure calculation.

For introducing magnetism in band structure calculation first spin-polarized calculations were done with various spin configurations. It was not possible to stabilize a Néel order (magnetic moments collapse), but the ferromagnetic (FM) and two antiferromagnetic phases, the stripy and the zig-zag phases, are all stable, with the ground state practically degenerate between the two AFM states (Fig. 7.13).[94] The FM state has a small advantage in the calculations, which is lost upon application of U (see Fig. 7.13). Spin-orbit coupling has very little effect to consider here due to small value in $4 \mathrm{~d}$. Hence spin-polarized calculation clearly suggest that ground state of $\mathrm{Li}_{2} \mathrm{RhO}_{3}$ is degenerate between stripy and the zigzag AF phases. 


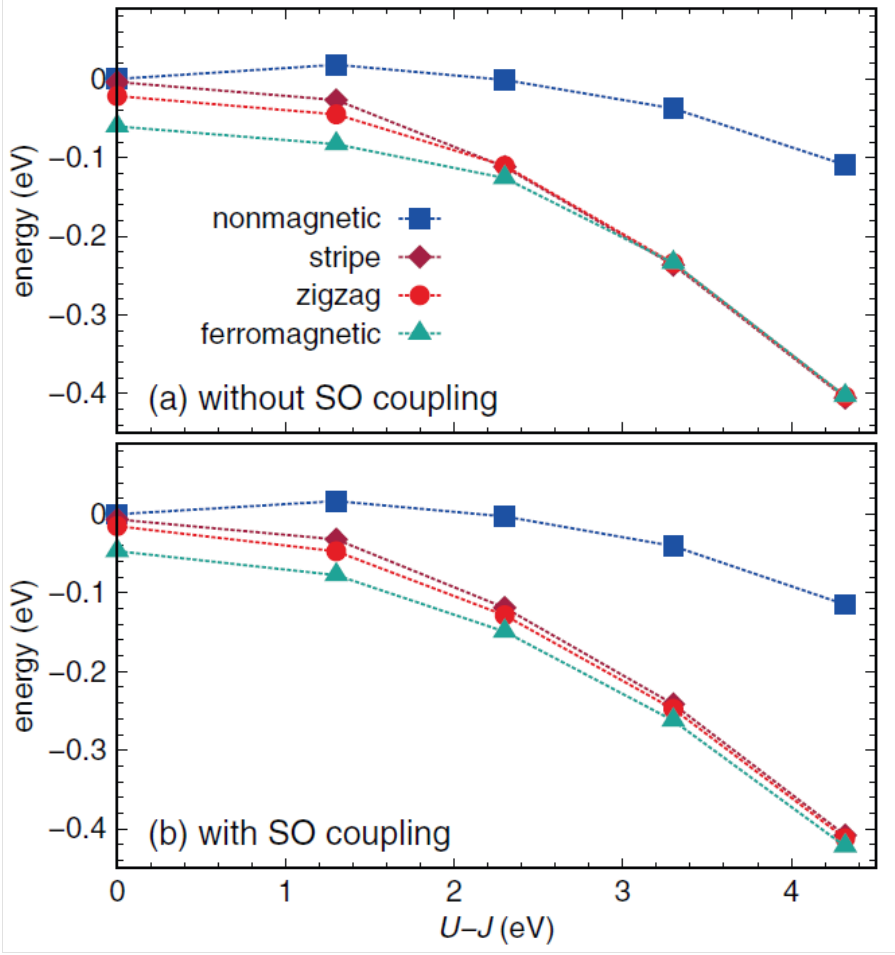

Figure 7.13: Energy of different magnetic configurations in $\mathrm{eV} / \mathrm{Rh}$ relative to nonmagnetic sate as a function of (UJ) (a) Without spin-orbit (SO) coupling. (b) With spin-orbit (SO) coupling. Reprinted from [94].

To find the real origin of the insulating behavior in $\mathrm{Li}_{2} \mathrm{RhO}_{3}$ density of states (DOS) were calculated by local density approximation (LDA) approach with and without SO; with and without $U$ for both stripy and the zig-zag spin configurations (Fig. 7.14 and 7.15) respectively). As expected even after including magnetism without $\mathrm{SO}$ and $U, \mathrm{Li}_{2} \mathrm{RhO}_{3}$ is metallic having huge DOS at $\mathrm{E}_{F}$ (see (a) in both Fig.) and even including SO coupling does not change much in DOS at $\mathrm{E}_{F}$ (see (e)in both Fig.), it is still highly metallic. This is very different from its Ir counterpart where DOS does change significantly after including SO [58]. Insulating gap only opens for both the configuration for $U \geq 3 \mathrm{eV}$ (see (b), (c), (f) and (g) in both Figure). It further enhances with increasing $U$ (see (d) and (h) in both Figure). However, one can not discard the effect of SO coupling. In nonmagnetic band structure calculation there is band crossing which is protected by symmetry and a SOC lifts that protection which help to realize the insulating state. So far exact band gap of $\mathrm{Li}_{2} \mathrm{RhO}_{3}$ is not known, hence we can not compare exact $U$ needed from DFT. But Insulating behavior clearly suggests that it must be smaller than $\mathrm{Na}_{2} \mathrm{IrO}_{3}$, where band gap is $340 \mathrm{meV}$. Comparing with that it can be predicted Coulomb repulsion in $\mathrm{Li}_{2} \mathrm{RhO}_{3} U$ is $3-4 \mathrm{eV}$ which is very reasonable for a $4 \mathrm{~d}$ material. From this DFT analysis it is very clear that Coulomb repulsion is playing a crucial role to open a gap in $\mathrm{Li}_{2} \mathrm{RhO}_{3}$ but whether this insulating behavior is coupled with AF correlation or not can not be said by DFT. DFT does not resolve whether it is band (Slater) insulator or a Mott insulator but very similar behavior 


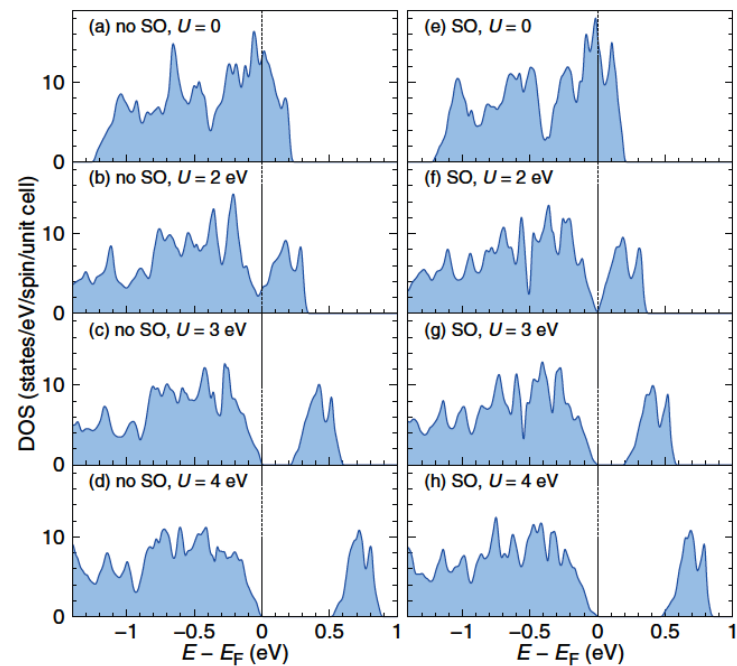

Figure 7.14: Evolution of density of states (DOS) with Hubbard U in zigzag phase without and with spin-orbit coupling (SO). Reprinted from [94].

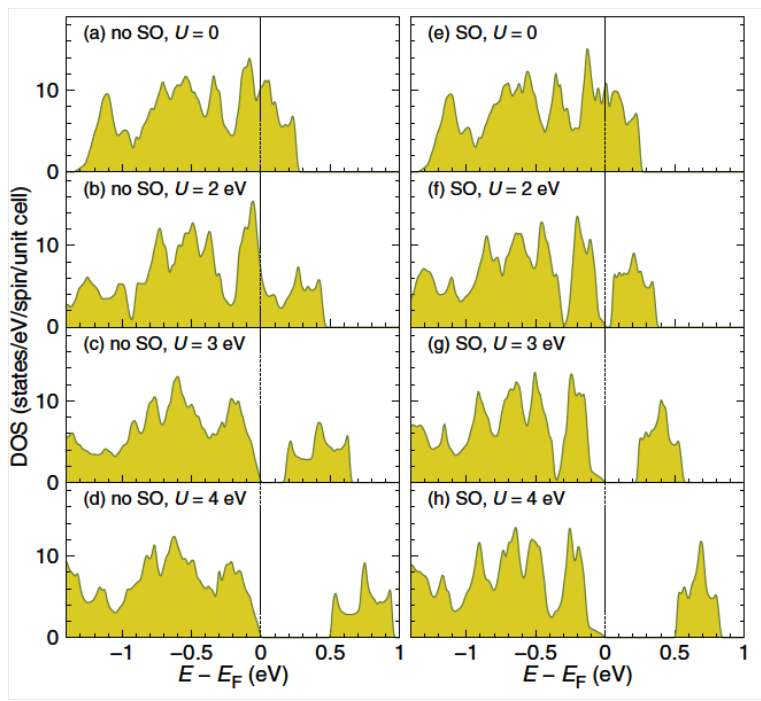

Figure 7.15: Evolution of density of states (DOS) with Hubbard U in stripy phase without and with spin-orbit coupling (SO). Reprinted from [94].

of resistivity in all these compounds down to lowest temperature indicates $\mathrm{Li}_{2} \mathrm{RhO}_{3}$ is also a Mott insulator.

\subsection{Discussion}

A rigorous study to find Kitaev spin liquid state helped to find this interesting material, honeycomb lattice rhodate $\mathrm{Li}_{2} \mathrm{RhO}_{3}$. This material is equally sensitive to disorder like its Ir-sister $\mathrm{Li}_{2} \mathrm{IrO}_{3}$. All the investigation was done on well ordered samples. Observed insulating behavior very categorically investigated by band structure calculation. It indicated $\mathrm{Li}_{2} \mathrm{RhO}_{3}$ is a one of the rare $4 \mathrm{~d}-$ Mott insulator where most likely honeycomb geometry of edge-sharing $\mathrm{RhO}_{6}$ octahedra a special ingredient for insulating QMO bands. $\mathrm{Li}_{2} \mathrm{RhO}_{3}$ electronic structure is formed by a delicate interplay between one-electron hopping and Coulomb repulsion $U$ with the assistance of small SO coupling where SOC and $U$ prevails to realize an insulating state.

Magnetic ground state was highly controversial to have a spin-glass freezing. But a spinglass freezing only happens in a frustrated spin-clusters having structural fault (here stacking fault). In $\mathrm{Li}_{2} \mathrm{RhO}_{3}$, a typical power-law behavior in heat capacity and spin-lattice relaxation rate strongly suggests some gapless excitation originated from degeneracy in magnetic ground state. Here again, spin-polarized calculations prove a strong degeneracy between the 
stripy and zig-zag spin-configuration. The cause of this degeneracy must be lying behind its frustrated interactions. If with small $\mathrm{SO}$ coupling $\mathrm{Li}_{2} \mathrm{RhO}_{3}$ has still $J_{\text {eff }}=1 / 2$ relativistic state then it could be a strongest candidate for Kitaev spin-liquid. Because unlike honeycomb lattice iridates where a fine tuning between isotropic Heisenberg interaction and anisotropic Kitaev interaction help to magnetically order them in a unconventional magnetic structure, $\mathrm{Li}_{2} \mathrm{RhO}_{3}$ does not order and shows degeneracy between different magnetic structures. A $J_{\text {eff }}=1 / 2$ state in $\mathrm{Li}_{2} \mathrm{RhO}_{3}$ would clearly indicate it is in the border of Kitaev Spin liquid state. But to verify $J_{\text {eff }}=1 / 2$ state, electronic structure of $\mathrm{Li}_{2} \mathrm{RhO}_{3}$ should be investigated by the resonant inelastic x-ray scattering similar to $\mathrm{A}_{2} \mathrm{IrO}_{3}$. If this gives a fruitful result then low energy excitations due to degenerate ground state can be studied by inelastic neutron scattering. 


\section{Chapter 8}

\section{Summary and Outlook}

Honeycomb lattice iridates $\mathrm{Na}_{2} \mathrm{IrO}_{3}$ and $\mathrm{Li}_{2} \mathrm{IrO}_{3}$ have been synthesized in single- and polycrystalline forms respectively, despite many challenges due to disorder. Obtaining phase pure $\mathrm{Li}_{2} \mathrm{IrO}_{3}$ with least amount of stacking disorder having long range magnetic ordering was one of the best achievement during the project. Structural characterization of $\mathrm{A}_{2} \mathrm{IrO}_{3}$ has been done by x-ray diffraction and pair distribution function analysis which unambiguously identified that both the honeycomb iridates crystallize in $\mathrm{C} 2 / \mathrm{m}$ crystal structure. In both systems, trigonal distortion of the $\mathrm{IrO}_{6}$ octahedra plays a significant role in controlling their magnetic property.

Magnetically, both the systems show long range antiferromagnetic ordering below $T_{N}$ $=15 \mathrm{~K}$ and an antiferromagnetic correlation between Ir $S_{\text {eff }}=1 / 2$ moments which is very strong in the Na-system $\left(\theta_{W}=-125 \mathrm{~K}\right)$ and weaker in the Li-system $\left(\theta_{W}=-40 \mathrm{~K}\right)$. Neutron scattering has identified that the Na-system has an unconventional zigzag magnetic ground state whereas the magnetic structure of the Li-system is of incommensurate spiral type. Both of these magnetic ground states can not be explained by the Heisenberg-Kitaev (HK) model with the ferromagnetic Kitaev exchange. To understand the possible physical model for honeycomb lattice iridates, different doping studies have been done.

Single crystal growth of $\mathrm{Li}_{2} \mathrm{IrO}_{3}$ was not successful so-far, hence to understand the change of magnetic ground state from the Na-system to the Li-system, $\left(\mathrm{Na}_{1-x} \mathrm{Li}_{x}\right)_{2} \mathrm{IrO}_{3}$ crystals were grown. For low doping $x \leq 0.25$, long range $\mathrm{AF}$ ordering temperature $T_{N}$ from $15 \mathrm{~K}$ to $5 \mathrm{~K}$ as well as $a$ and $b$ lattice parameters strongly decrease which suggests strong change in the shape of Ir-honeycomb. This can be a useful pathway to search theoretically predicted topologically non-trivial phases in honeycomb lattice iridates. Presently $\left(\mathrm{Na}_{1-x} \mathrm{Li}_{x}\right)_{2} \mathrm{IrO}_{3}$ crystals of this region are investigated by surface sensitive probes like 
ARPES and STS and primary results give hint of significant change in the electronic properties. Changes in magnetic structure will also be investigated by resonant x-ray magnetic scattering (RXMS). Unfortunately, for $x>0.25$ a miscibility gap is observed in the $\left(\mathrm{Na}_{1-x} \mathrm{Li}_{x}\right)_{2} \mathrm{IrO}_{3}$ phase diagram which is experimentally investigated by x-ray diffraction, scanning tunneling spectroscopy, magnetization and heat capacity and theoretically investigated by DFT calculations. Theoretical study revealed that for $x \leq 0.25 \mathrm{Li}$ occupies only the Na-site in the honeycomb layer which explains the pronounced decrease of $a$ and $b$ lattice parameters. For $x>0.25, \mathrm{Li}$ atoms also occupy Na-positions in the Na-layers, resulting in a clustering of $\mathrm{Li}$ in the Na-layer and energetically unstable structure. Finding a miscibility gap in the $\left(\mathrm{Na}_{1-x} \mathrm{Li}_{x}\right)_{2} \mathrm{IrO}_{3}$ phase diagram is one of the useful findings in this thesis work.

Doping the Ir site has revealed many important properties of honeycomb iridates. A magnetic impurity doping at $\operatorname{Ir} S=1 / 2$ site gives spin-glass freezing below a particular temperature, $6 \mathrm{~K}$. But the systematic dilution of Ir $S_{\text {eff }}=1 / 2$ moment by nonmagnetic Ti substitution systematically varies the $\mathrm{CW}$ temperature and the spin-freezing temperature $T_{g}$. $\theta_{W}$ study on $\mathrm{Na}_{2}\left(\operatorname{Ir}_{1-x} \mathrm{Ti}_{x}\right) \mathrm{O}_{3}$ single crystals show that Ir-magnetism (its CW scale) is drastically destroyed with the increasing dilution below the percolation threshold $\left(x_{p}=\right.$ 0.303) of the nearest neighbor interactions in honeycomb iridates. Also $T_{g}$ linearly decreases towards zero below $x_{p}$. This indicates that the magnetic exchange in $\mathrm{Na}_{2} \mathrm{IrO}_{3}$ is dominated by nearest neighbor interactions. Analysis with different HK models suggests that nearest neighbor HK model with the FM Kitaev, AF Heisenberg interaction and an anisotropic interaction term due to the trigonal distortion of the $\mathrm{IrO}_{6}$ octahedra successfully explains the zigzag magnetic ground states of $\mathrm{Na}_{2} \mathrm{IrO}_{3}$. By controlling the trigonal distortion a Kitaev spin liquid may be possible to achieve in $\mathrm{Na}_{2} \mathrm{IrO}_{3} \cdot[89]$

Our nonmagnetic dilution study in Li-system with $\mathrm{Li}_{2}\left(\operatorname{Ir}_{1-x} \mathrm{Ti}_{x}\right) \mathrm{O}_{3}$ polycrystal revealed an even bigger surprise. A spin-glass freezing is observed even beyond 50\% nonmagnetic dilution. Even there, the CW-scale has not gone to zero. Moreover, a field induced quantum phase transition is observed in magnetic Grüneisen measurements for $x=0.51$ and 0.55 $\mathrm{Li}_{2}\left(\mathrm{Ir}_{1-x} \mathrm{Ti}_{x}\right) \mathrm{O}_{3}$ samples. This suggests that in the Li-system, beyond nearest neighbor exchange interactions are very significant. A rigorous analysis of $\mathrm{Li}_{2} \mathrm{IrO}_{3}$ magnetism with different HK models concludes that the HK model with the $2^{\text {nd }}$ nearest neighbor FM Heisenberg and the AF Kitaev interaction should be the ideal description for the Li-honeycomb iridate. The observed field-induced quantum phase transition in $\operatorname{Li}_{2}\left(\operatorname{Ir}_{1-x} \mathrm{Ti}_{x}\right) \mathrm{O}_{3}$ may be similar to the quantum Griffiths phase and needs to be microscopically investigated.

The desired Kitaev spin liquid state has not yet been found in honeycomb lattice iridates. A ray of hope is found in the honeycomb lattice rhodate $\mathrm{Li}_{2} \mathrm{RhO}_{3}$ where a signature 
for a degenerate ground state is observed in heat capacity and NMR measurement as well as in spin-polarized DFT calculation but the magnetic measurements show a spin-glass freezing below $T_{g}=6 \mathrm{~K}$ due to higher order of stacking fault in the Rh-honeycomb stacking. The origin of this degenerate ground state needs to be more intensely investigated by inelastic neutron scattering. A very newly found $\beta-\mathrm{Li}_{2} \mathrm{IrO}_{3}$ phase with three dimensional network of edge sharing octahedra shows another pathway to look for the Kitaev spin-liquid state.

This thesis work has given a deep insight into the physics of honeycomb lattice iridates and rhodtaes as well as future directions to look for the desired Kitaev spin-liquid state and possible topologically non-trivial state in honeycomb iridates. Honeycomb iridates $\mathrm{A}_{2} \mathrm{IrO}_{3}$ are layered Mott insulators like $\mathrm{La}_{2} \mathrm{CuO}_{4}$ which shows high-temperature superconductivity by charge carrier doping. Hence $\mathrm{A}_{2} \mathrm{IrO}_{3}$ also posses significant possibilities to realize exotic electronic sates like anomalous metallic state, a superconducting state or even a topological superconducting state under charge carrier doping.[100] 



\section{Appendix A}

\section{Preliminary result of $\beta-\mathrm{Li}_{2} \mathrm{IrO}_{3}$}

At the very end of thesis work, $\beta-\mathrm{Li}_{2} \mathrm{IrO}_{3}$ phase was identified as the high temperature phase of $\mathrm{Li}_{2} \mathrm{IrO}_{3}$. It was sintered at high temperature at $1080^{\circ} \mathrm{C}$, the PXRD peak at $19^{\circ}$ gradual increases with increasing $T$, but peaks from honeycomb- $\mathrm{Li}_{2} \mathrm{IrO}_{3}$ phase still existed. Then 10 atomic\% $\mathrm{IrO}_{2}$ was added with the honeycomb- $\mathrm{Li}_{2} \mathrm{IrO}_{3}$ and sintered at $1100{ }^{\circ} \mathrm{C}$ for 72 days and then air quenched. It resulted a single phase $\beta-\mathrm{Li}_{2} \mathrm{IrO}_{3}$.

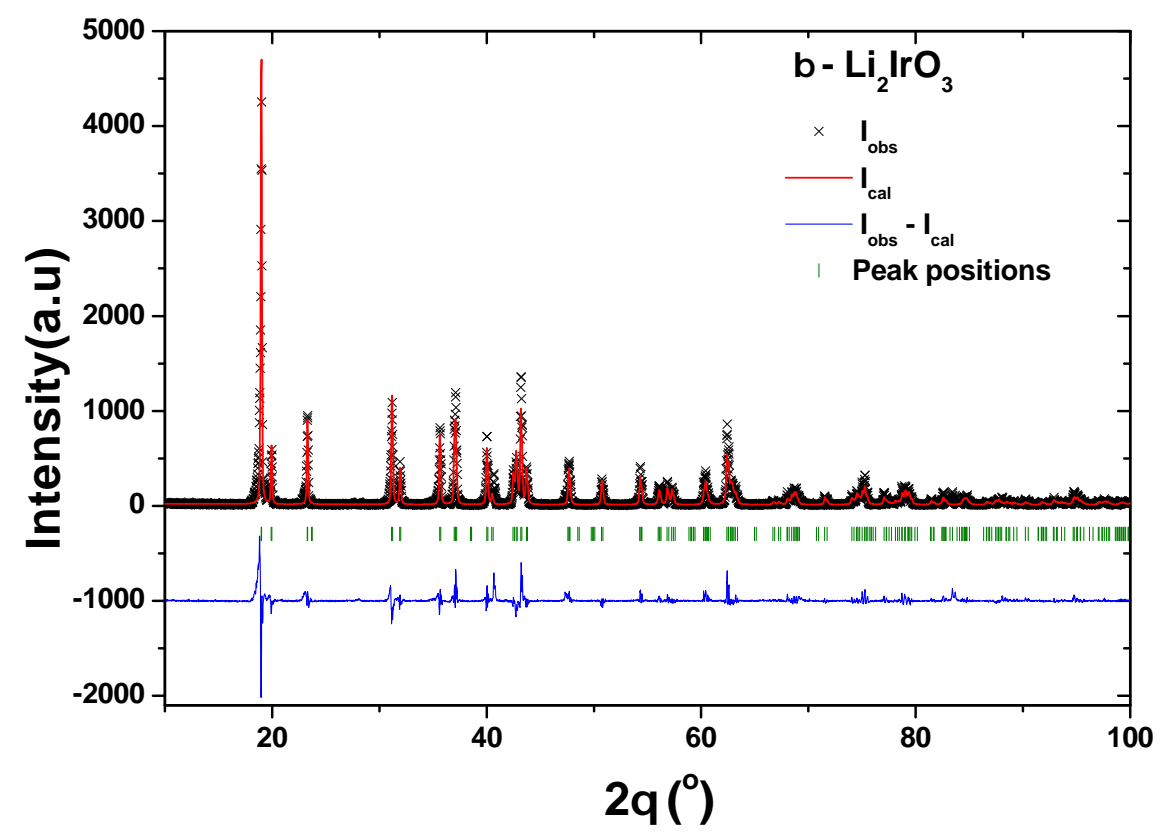

Figure A.1: Powder XRD pattern of $\beta-\mathrm{Li}_{2} \mathrm{IrO}_{3}(\mathrm{X}$ marks) with theoretical fitting by Rietveld analysis (red line). 


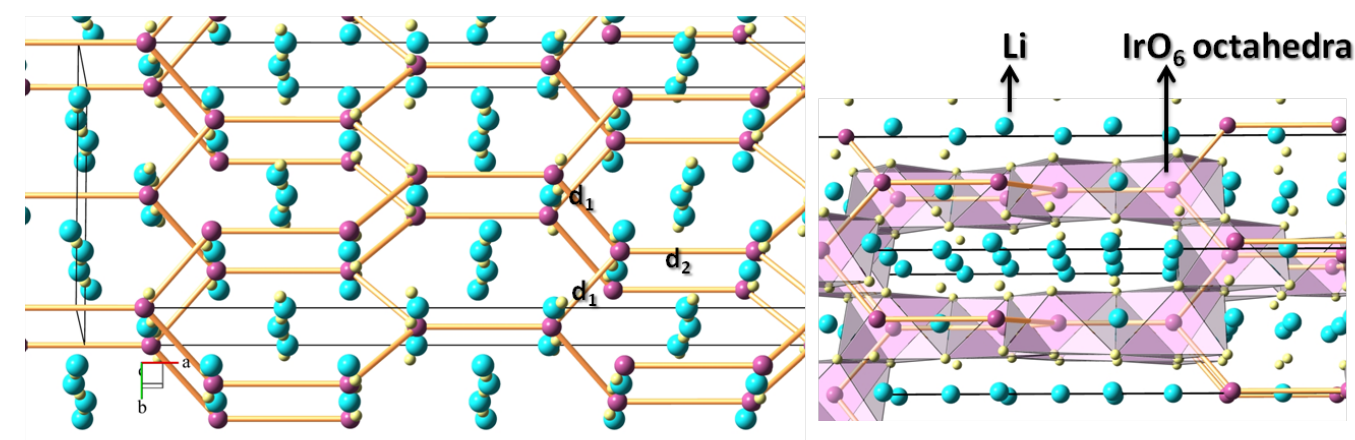

Figure A.2: Crystal structure of $\beta-\mathrm{Li}_{2} \mathrm{IrO}_{3}$.

Table A.1: Structural parameters of $\beta-\mathrm{Li}_{2} \mathrm{IrO}_{3}$ in Fddd space group(No. 70) obtained from Rietveld analysis. Fitting quality parameters $\mathrm{R}_{p}$ and $\mathrm{R}_{w p}=0.21$ and 0.28 respectively.

\begin{tabular}{l|l|l|l|l|l|l}
\hline atom & site & $x$ & $y$ & $z$ & $O c c$. & $B$ \\
\hline $\mathrm{Ir}$ & $16 \mathrm{e}$ & $1.04231(15)$ & $1 / 8$ & $1 / 8$ & 1 & 0.005 \\
$\mathrm{Li}$ & $16 \mathrm{e}$ & $0.2960(11)$ & $1 / 8$ & $1 / 8$ & 1 & 0.006 \\
$\mathrm{Li}$ & $16 \mathrm{e}$ & $0.533(4)$ & $1 / 8$ & $1 / 8$ & 1 & 0.006 \\
$\mathrm{O}$ & $32 \mathrm{~h}$ & $1 / 8$ & $8.880(7)$ & $1 / 8$ & 1 & 0.007 \\
$\mathrm{O}$ & $16 \mathrm{f}$ & $0.886(4)$ & $1 / 8$ & $1 / 8$ & 1 & 0.007 \\
\hline
\end{tabular}

PXRD pattern can be fitted with the reported Fddd orthorhombic (Space group no. 70, $\mathrm{Z}=16$ ) crystal structure [71]. Fig. A.1 shows Rietveld analysis of the PXRD pattern with this crystal structure. All the peaks are well indexed by Fddd crystal structure and resulting atomic parameters are listed in Table. A.1. Lattice parameters are: $\mathrm{a}=17.8040(8) \AA$, $\mathrm{b}=5.90533(29) \AA$ 列 $\mathrm{c}=8.4531(4) \stackrel{\circ}{A}$. Crystal structure is depicted in Fig. A.2. It is no more a layered structure of $\mathrm{IrO}_{6}$ honeycomb, rather a $3 \mathrm{D}$ network of edge sharing $\mathrm{IrO}_{6}$ octahedra (right). But local structure of $\beta$-phase is very similar to $\mathrm{Li}_{2} \mathrm{IrO}_{3}$, three edge sharing $\mathrm{IrO}_{6}$ octahedra forms Ir-O-Ir bond angle as well as three different exchange path. Two Ir-Ir bond angles are same $d_{1}=2.945(8) \AA$ another is little bigger $d_{2}=2.9858(27) \AA$. Here honeycomb is not directly connected, rather toplogically connected. Due to its similar kind of exchange path between two neighboring Ir this structure seems still relevant for Kitaev physics.

Magnetization was measured on well ordered single-phase $\beta-\mathrm{Li}_{2} \mathrm{IrO}_{3}$ polycrystal at $\mathrm{H}=1 \mathrm{~T}$. A jump in susceptibility is observed around $40 \mathrm{~K}$ like ferromagnetic material (Fig. A.3). But CW fit on inverse susceptibility gives Weiss temperature, $\theta_{W}=-7(10) \mathrm{K}$. Near zero Weiss temperature indicates a higher dynamical frustration between due to FM Kitaev interaction. Concurrently, more detailed research is going on in different types of polymorph of 


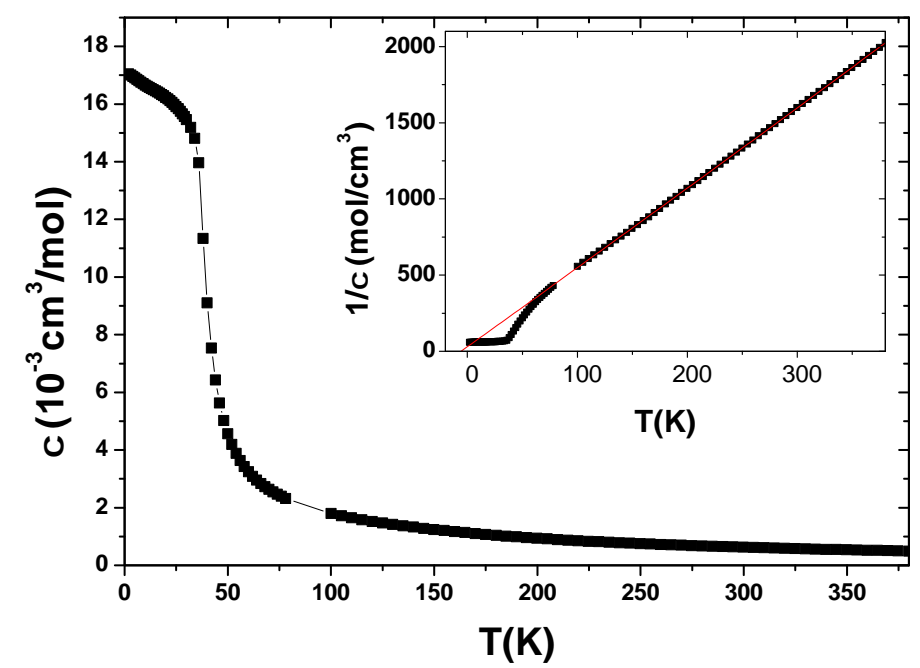

Figure A.3: $\chi$ versus $\mathrm{T}$ for $\beta$ - $\mathrm{Li}_{2} \mathrm{IrO}_{3}$. (inset) $\mathrm{CW}$ fitting of inverse $\chi$, intersects near zero.

$\mathrm{Li}_{2} \mathrm{IrO}_{3}$ by other groups and our group as well.[71, 101] 



\section{Appendix B}

\section{Structural analysis of Li-Rh-O disordered phases}

Two types of disordered $\mathrm{Li}_{2} \mathrm{RhO}_{3}$ phases are obtained. Both of the disordered phase are identified as different polymorph of $\mathrm{LiRhO}_{2}$. Two phases are: (1) $\mathrm{Li}_{2} \mathrm{RhO}_{3}$ sintered at high temperature, $970^{\circ} \mathrm{C}$. (2) $\mathrm{Li}_{2} \mathrm{RhO}_{3}$ treated with $\mathrm{LiCl}$ flux, resulted $\mathrm{LiRhO}_{2}$. Two phases are identified by Rietveld fitting of the measured powder XRD data.[46, 47]

Table B.1: Crystal structure of $\mathrm{Li}_{2} \mathrm{RhO}_{3}$ disordered phase: $\mathrm{LiRhO}_{2}, \mathrm{Li}_{2} \mathrm{RhO}_{3}$ sintered at $970^{\circ} \mathrm{C}$. Space group: $\mathrm{F} \mathrm{d} \overline{3} \mathrm{~m}$ (space group number 227 ). Lattice parameters : $a=b=c=$ 8.39208(30) $\AA$; $\alpha=\beta=\gamma=90^{\circ}$

\begin{tabular}{l|l|l|l|l}
\hline atom & $x$ & $y$ & $z$ & Occ. \\
\hline $\mathrm{O}$ & $0.2530(6)$ & $0.2530(6)$ & $0.2530(6)$ & 1 \\
$\mathrm{Li}$ & 0.5 & 0.5 & 0.5 & 1 \\
$\mathrm{Rh}$ & 0 & 0 & 0 & 1 \\
\hline
\end{tabular}

Table B.2: Crystal structure of $\mathrm{Li}_{2} \mathrm{RhO}_{3}$ disordered phase: $\mathrm{LiRhO}_{2}, \mathrm{Li}_{2} \mathrm{RhO}_{3}$ sintered with $\mathrm{LiCl}$ (SM12S). Space group: $\mathrm{R} \overline{3} \mathrm{~m}: \mathrm{h}$ (space group no. 166. Lattice parameters : $a=b=3.0106(18) \AA$ A, $c=14.328(8) \AA$; $\alpha=\beta=90^{\circ} \gamma=120^{\circ}$

\begin{tabular}{l|l|l|l|l}
\hline atom & $x$ & $y$ & $z$ & Occ. \\
\hline $\mathrm{O}$ & 0 & 0 & $0.2682(8)$ & 1 \\
$\mathrm{Li}$ & 0 & 0 & 0 & 1 \\
$\mathrm{Rh}$ & 0 & 0 & 0 & 1 \\
\hline
\end{tabular}




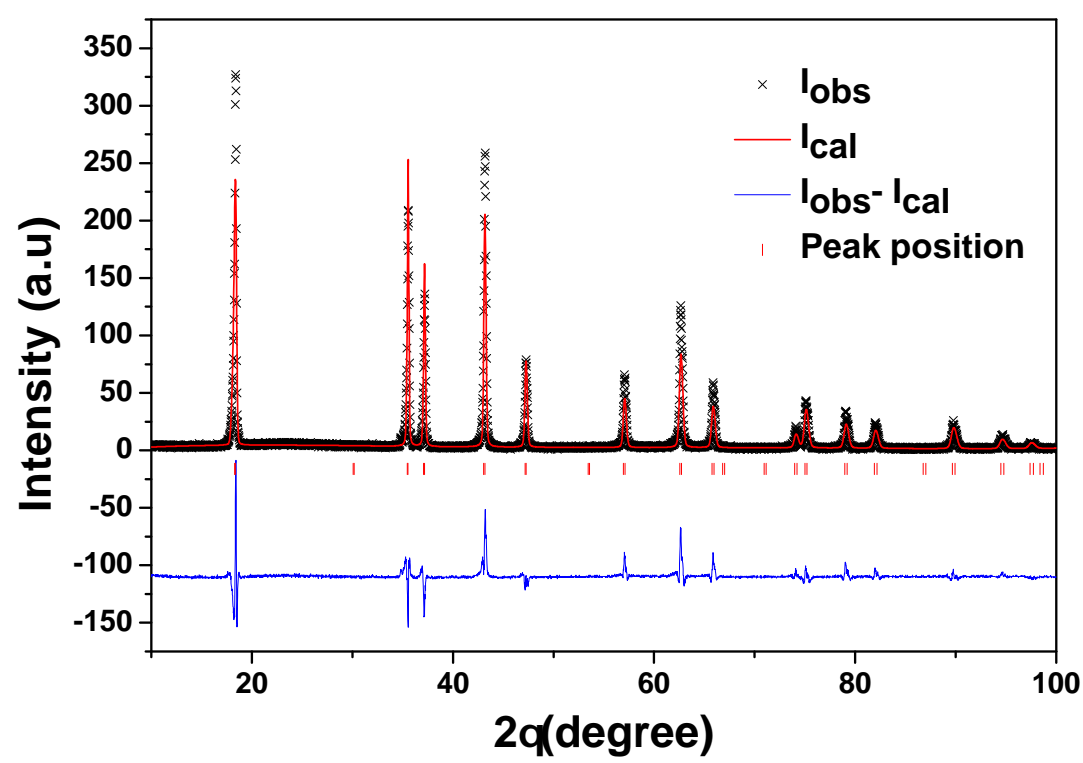

Figure B.1: Rietveld refinement of XRD data of polycrystalline $\mathrm{Li}_{2} \mathrm{RhO}_{3}$ synthesized at $970^{\circ} \mathrm{C} . I_{o b s}$ is the experimentally obtained data, $I_{o b s}$ is obtained from Riteveld fitting.

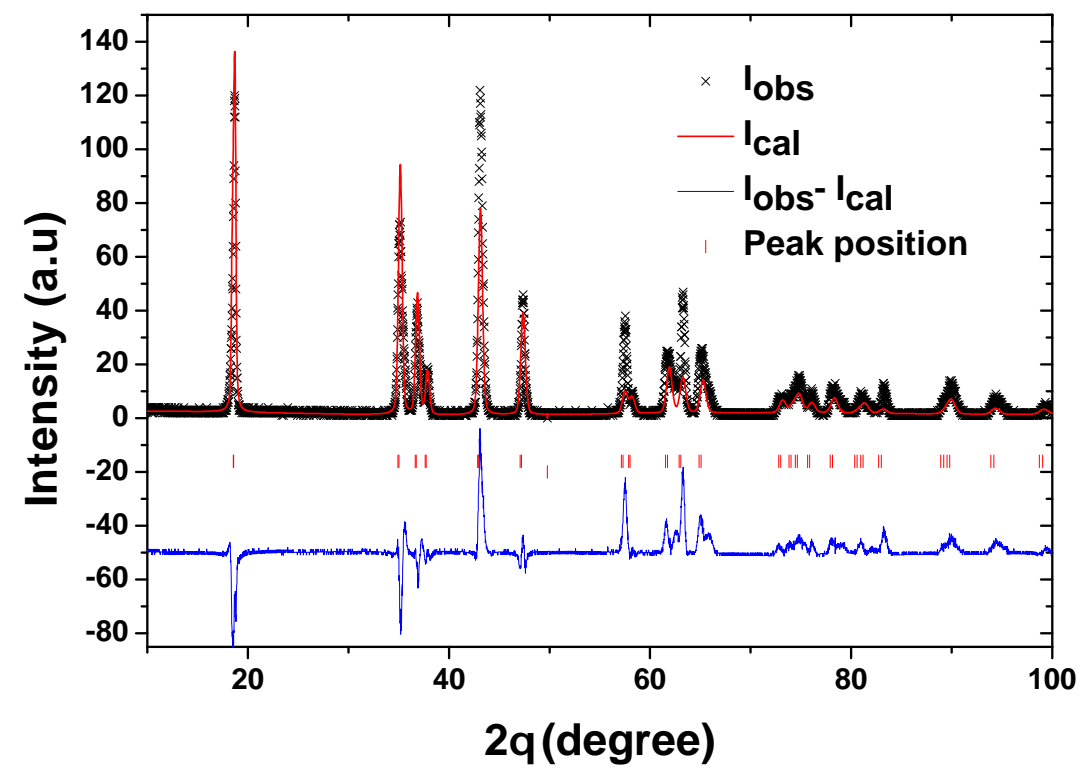

Figure B.2: Rietveld refinement of XRD data of polycrystalline $\mathrm{Li}_{2} \mathrm{RhO}_{3}$ synthesized with $\mathrm{LiCl}(\mathrm{SM} 12 \mathrm{~S}) . I_{o b s}$ is the experimentally obtained data, $I_{o b s}$ is obtained from Riteveld fitting. 


\section{Bibliography}

[1] David C. Johnston. Handbook of Magnetic Materials, edited by K. H. J. Buschow, volume 10. Elsevier, 1997.

[2] T. A. Kaplan and S. D. Mahanti, editors. Physics of Manganites. Fundamental Materials Research. Kluwer Academic Publishers, Boston, 2002.

[3] Yoshitaka Matsushita, Hiroaki Ueda, and Yutaka Ueda. Flux crystal growth and thermal stabilities of $\mathrm{LiV}_{2} \mathrm{O} 4$. Nat. Mater., 4(11):845-850, 2005.

[4] Andrew Peter Mackenzie. The superconductivity of $\mathrm{Sr}_{2} \mathrm{RuO}_{4}$ and the physics of spintriplet pairing. Rev. Mod. Phys., 75(2):657-712, 2003.

[5] P. Gegenwart et. al. Metamagnetic Quantum Criticality in $\mathrm{Sr}_{3} \mathrm{Ru}_{2} \mathrm{O}_{7}$ Studied by Thermal Expansion. Phys. Rev. Lett., 96(13):136402, 2006.

[6] R A Borzi et. al. Formation of a nematic fluid at high fields in $\mathrm{Sr}_{3} \mathrm{Ru}_{2} \mathrm{O}_{7}$. Science, 315(5809):214-7, 2007.

[7] C. L. Kane and E. J. Mele. $\mathrm{Z}_{2}$ Topological Order and the Quantum Spin Hall Effect. Phys. Rev. Lett., 95(14):146802, 2005.

[8] D Hsieh et. al. A topological Dirac insulator in a quantum spin Hall phase. Nature, 452(7190):970-4, 2008.

[9] Dmytro Pesin and Leon Balents. Mott physics and band topology in materials with strong spinorbit interaction. Nat. Phys., 6(5):376-381, 2010.

[10] B . Kim et. al. Novel $J_{\text {eff }}=1 / 2$ Mott State Induced by Relativistic Spin-Orbit Coupling in $\mathrm{Sr}_{2} \mathrm{IrO}_{4}$. Phys. Rev. Lett., 101(7):076402, 2008.

[11] Alexei Kitaev. Anyons in an exactly solved model and beyond. Ann. Phys. (N. Y)., $321(1): 2-111,2006$.

[12] Hong-Chen Jiang et. al. Possible proximity of the Mott insulating iridate $\mathrm{Na}_{2} \mathrm{IrO}_{3}$ to a topological phase: Phase diagram of the Heisenberg-Kitaev model in a magnetic field. Phys. Rev. B, 83(24):245104, 2011.

[13] Jirí Chaloupka, George Jackeli, and Giniyat Khaliullin. Kitaev-Heisenberg Model on a Honeycomb Lattice: Possible Exotic Phases in Iridium Oxides $\mathrm{A}_{2} \mathrm{IrO}_{3}$. Phys. Rev. Lett., 105(2):027204, 2010. 
[14] Atsuo Shitade, Hosho Katsura, Jan Kuneš, Xiao-Liang Qi, Shou-Cheng Zhang, and Naoto Nagaosa. Quantum Spin Hall Effect in a Transition Metal Oxide $\mathrm{Na}_{2} \mathrm{IrO}_{3}$. Phys. Rev. Lett., 102(25):256403, 2009.

[15] N. W Ashcroft and N. D Mermin. Solid State Physics. Brooks/Cole, 1976.

[16] Patrik Fazekas. Lecture Notes on Electron Correlation and Magnetism. World Scientific Publishing Company, 1999.

[17] Masatoshi Imada, Atsushi Fujimori, and Yoshinori Tokura. Metal-insulator transitions. Rev. Mod. Phys., 70(4):1039-1263, 1998.

[18] Y. Tokura. Orbital Physics in Transition-Metal Oxides. Science (80), 288(5465):462$468,2000$.

[19] Sadamichi Maekawa. Physics of Transition Metal Oxides. Springer Series in Solid State Sciences, 2004.

[20] Coordination Compounds. http://www.rsc.org/pdf/tct/df-chapter.pdf, pages 54-70, 2005.

[21] Masahito Mochizuki and Masatoshi Imada. Orbital physics in the perovskite Ti oxides. New J. Phys., 6(1):154-154, 2004.

[22] J. Clancy et. al. Spin-orbit coupling in iridium-based 5d compounds probed by x-ray absorption spectroscopy. Phys. Rev. B, 86(19):195131, 2012.

[23] Myron Salamon and Marcelo Jaime. The physics of manganites: Structure and transport. Rev. Mod. Phys., 73(3):583-628, 2001.

[24] Y. Tokura et. al. Colossal magnetoresistive manganites. J. Magn. Magn. Mater., 200(1-3):1-23, 1999.

[25] Jean-Michel Delisle Carter Carter. Interplay between spin-orbit coupling, electronic correlations and lattice distortions in perovskite iridates. PhD thesis, 2013.

[26] Hlynur Gretarsson. X-ray Spectroscopy Studies of Iridates and Iron Based Superconductors. PhD thesis, University of Toronto, 2013.

[27] G. Jackeli and G. Khaliullin. Mott Insulators in the Strong Spin-Orbit Coupling Limit: From Heisenberg to a Quantum Compass and Kitaev Models. Phys. Rev. Lett., 102(1):017205, 2009.

[28] M. Crawford et. al. Structural and magnetic studies of $\mathrm{Sr}_{2} \mathrm{IrO}$. Phys. Rev. B, 49(13):9198-9201, 1994.

[29] J. P. L. Faye et. al. Topological phases of the Kitaev-Hubbard model at half filling. Phys. Rev. B, 89(11):115130, 2014.

[30] Xiao-Yong Feng et. al. Topological Characterization of Quantum Phase Transitions in a Spin-1/2 Model. Phys. Rev. Lett., 98(8):087204, 2007.

[31] Saptarshi Mandal et. al. Exactly solvable Kitaev model in three dimensions. Phys. Rev. B, 79(2):024426, 2009. 
[32] Shuo Yang et. al. Fidelity susceptibility and long-range correlation in the Kitaev honeycomb model. Phys. Rev. A, 78(1):012304, 2008.

[33] Jií Chaloupka, George Jackeli, and Giniyat Khaliullin. Zigzag Magnetic Order in the Iridium Oxide . Phys. Rev. Lett., 110(9):097204, 2013.

[34] Itamar Kimchi and Yi-Zhuang You. Kitaev-Heisenberg-J_\{2\}-J_\{3\} model for the iridates $\mathrm{A}_{2} \mathrm{IrO}_{3}$. Phys. Rev. B, 84(18):180407, 2011.

[35] Johannes Reuther, Ronny Thomale, and Stephan Rachel. Spiral order in the honeycomb iridate $\mathrm{Li}_{2} \mathrm{IrO}_{3}$. arXiv : 1404.5818, page 5, 2014.

[36] S. K. Choi, R. Coldea, A. N. Kolmogorov, T. Lancaster, I. I. Mazin, S. J. Blundell, P. G. Radaelli, Yogesh Singh, P. Gegenwart, K. R. Choi, S.-W. Cheong, P. J. Baker, C. Stock, and J. Taylor. Spin Waves and Revised Crystal Structure of Honeycomb Iridate $\mathrm{Na}_{2} \mathrm{IrO}_{3}$. Phys. Rev. Lett., 108(12):127204, 2012.

[37] E. Rastelli et. al. Non-simple magnetic order for simple Hamiltonians. Phys. $B+C$, 97(1):1-24, 1979.

[38] E. Rastelli. Statistical Mechanics of Magnetic Excitations: From Spin Waves to Stripes and Checkerboards. World Scientific Publishing Company, 2013.

[39] J.B. Fouet et. al. An investigation of the quantum $\mathrm{J}_{1}-\mathrm{J}_{2}-\mathrm{J}_{3}$ model on the honeycomb lattice. Eur. Phys. J. B, 20(2):241-254, 2001.

[40] I. I. Mazin et. al. $\mathrm{Na}_{2} \mathrm{IrO}_{3}$ as a Molecular Orbital Crystal. Phys. Rev. Lett., 109(19):197201, 2012.

[41] A. R. West. Solid state chemistry and its applications. John Wiley and Sons, 1984.

[42] J. C. Brice. Crystal growth Processes. John Wiley and Sons, 1986.

[43] G. Dhanraj, K. Byrappa, V. Prasad, and M. Dudley, editors. Handbook of Crystal Growth. Springer.

[44] Introduction to Energy Dispersive X-ray Spectrometry (EDS).

[45] B. D. Cullity. Elements of X-Ray Diffraction. Addison-Wesley Publishing company Inc., second edition.

[46] A.C Larson and Dreele R. B. Von. General Structure Analysis System (GSAS). Technical report, Los Alamos National Laboratory, 2000.

[47] Brian H. Toby. EXPGUI, a graphical user interface for GSAS. J. Appl. Crystallogr., $34(2): 210-213,2001$.

[48] Quantum Design. Magnetic Property Measurement System manual, Part number 1004-100A. Technical report.

[49] Quantum Design. Physical Property Measurement System Heat capacity option User's manual, Part number 1085-150, H-1. Technical report. 
[50] Quantum Design. Physical Property Measurement System Hardware manual, Part Number 1070-150, B-1. Technical report.

[51] Quantum Design. Physical Property Measurement System Resistivity option User's manual, Part number 1076-100A. Technical report.

[52] Yogesh Singh and P. Gegenwart. Antiferromagnetic Mott insulating state in single crystals of the honeycomb lattice material $\mathrm{Na}_{2} \mathrm{IrO}_{3}$. Phys. Rev. B, 82(6):064412, 2010.

[53] I. Felner et. al. The magnetic behavior of $\mathrm{Li}_{2} \mathrm{MO}_{3}(\mathrm{M}=\mathrm{Mn}, \mathrm{Ru}$ and $\mathrm{Ir})$ and Li2(Mn1xRux)O3. Phys. B Condens. Matter, 311(3-4):195, 2002.

[54] Hironori Kobayashi et. al. Structure, and magnetic and electrochemical properties of layered oxides, $\mathrm{Li}_{2} \mathrm{IrO}_{3}$. J. Mater. Chem., 13(4):957, 2003.

[55] Matthew J. OMalley, Henk Verweij, and Patrick M. Woodward. Structure and properties of ordered $\mathrm{Li}_{2} \mathrm{IrO}_{3}$ and $\mathrm{Li}_{2} \mathrm{PtO}_{3}$. J. Solid State Chem., 181(8):1803, 2008.

[56] Matthew John O Malley. Matthew J . O' Malley Thesis, The magnetic behavior of $\mathrm{Li}_{2} \mathrm{MO}_{3}(\mathrm{M}=\mathrm{Mn}, \mathrm{Ru}$ and Ir). 3, 2009.

[57] Feng Ye et. al. Direct evidence of a zigzag spin-chain structure in the honeycomb lattice: A neutron and x-ray diffraction investigation of single-crystal $\mathrm{Na}_{2} \mathrm{IrO}_{3}$. Phys. Rev. B, 85(18):180403, 2012.

[58] H. Gretarsson, J. Clancy, X. Liu, J. Hill, Emil Bozin, Yogesh Singh, S. Manni, P. Gegenwart, Jungho Kim, A. Said, D. Casa, T. Gog, M. Upton, Heung-Sik Kim, J. Yu, Vamshi Katukuri, L. Hozoi, Jeroen van den Brink, and Young-June Kim. Crystal-Field Splitting and Correlation Effect on the Electronic Structure of $\mathrm{A}_{2} \mathrm{IrO}_{3}$. AND Supplemental Material. Phys. Rev. Lett., 110(7):076402, 2013.

[59] Simon J. L. Billinge. Pair Distribution Function Technique: Principles and Methods. In 43rd 44th Courses Struct. Minute Cryst. with Except. Prop. Front. Powder Diffr., Sicily, 2011. Erice Crystallography 2011 IT Support.

[60] Yogesh Singh, S. Manni, J. Reuther, T. Berlijn, R. Thomale, W. Ku, S. Trebst, and P. Gegenwart. Relevance of the Heisenberg-Kitaev Model for the Honeycomb Lattice Iridates $\mathrm{A}_{2} \mathrm{IrO}_{3}$. Phys. Rev. Lett., 108(12):127203, 2012.

[61] R. Comin et. al. $\mathrm{Na}_{2} \mathrm{IrO}_{3}$ as a Novel Relativistic Mott Insulator with a 340-meV Gap. Phys. Rev. Lett., 109(26):266406, 2012.

[62] N S Kini et. al. Transport and thermal properties of weakly ferromagnetic $\mathrm{Sr}_{2} \mathrm{IrO}_{4}$. J. Phys. Condens. Matter, 18(35):8205-8216, 2006.

[63] G. Cao et. al. Weak ferromagnetism, metal-to-nonmetal transition, and negative differential resistivity in single-crystal $\mathrm{Sr}_{2} \mathrm{IrO}_{4}$. Phys. Rev. B, 57(18):R11039-R11042, 1998.

[64] Jin-Ke Bao et. al. Variable range hopping conductivity and spin glass behavior in spin-ladder $\mathrm{Ba}_{0.6} \mathrm{~K}_{0.4} \mathrm{Fe}_{2} \mathrm{Se}_{3}$ single crystals. J. Phys. Condens. Matter, 26(2):026002, 2014. 
[65] Dong Yu et. al. Variable range hopping conduction in semiconductor nanocrystal solids. Phys. Rev. Lett., 92(21):216802, 2004.

[66] N. F. Mott. Conduction in non-crystalline materials. Philos. Mag., 19(160):835-852, 1969.

[67] N. Rogado et. al. $\mathrm{BaNi}_{2} \mathrm{~V}_{2} \mathrm{O}_{8}$ : A two-dimensional honeycomb antiferromagnet. Phys. Rev. B, 65(14):144443, 2002.

[68] Private communication with Dr. Kataev, EPR group head in IFW Dresdren. He is doing ESR measurement on $\mathrm{Li}_{2} \mathrm{IrO}_{3}$ and $\mathrm{Na}_{2} \mathrm{IrO}_{3}$. Technical report, 2013.

[69] Private communication with P. Khuntia on NMR results of $\mathrm{Li}_{2} \mathrm{IrO}_{3}$ and $\mathrm{Na}_{2} \mathrm{IrO}_{3}$ measured on the polycrystals prepared by myself. Technical report, 2013.

[70] Julien Bréger et. al. High-resolution X-ray diffraction, DIFFaX, NMR and first principles study of disorder in the $\mathrm{Li}_{2} \mathrm{MnO}_{3} \mathrm{Li}\left[\mathrm{Ni}_{1 / 2} \mathrm{Mn}_{1 / 2}\right] \mathrm{O}_{2}$ solid solution. J. Solid State Chem., 178(9):2575-2585, 2005.

[71] Tomohiro Takayama, Akihiko Kato, Robert Dinnebier, Jürgen Nuss, and Hidenori Takagi. Hyper-honeycomb iridate. arXiv : 11403.3296.

[72] X. Liu, T. Berlijn, W.-G. Yin, W. Ku, A. Tsvelik, Young-June Kim, H. Gretarsson, Yogesh Singh, P. Gegenwart, and J. P. Hill. Long-range magnetic ordering in $\mathrm{Na}_{2} \mathrm{IrO}_{3}$. Phys. Rev. B, 83(22):220403, 2011.

[73] S. K Choi and Radu Coldea. $\mathrm{Li}_{2} \mathrm{IrO}_{3}$ nutron scattering result. Private communication with Dr. Radu Coldea. Technical report, 2014.

[74] S. Manni, Sungkyun Choi, I. I. Mazin, R. Coldea, Michaela Altmeyer, Harald O. Jeschke, Roser Valentí, and P. Gegenwart. Effect of isoelectronic doping on the honeycomb-lattice iridate $\mathrm{A}_{2} \mathrm{IrO}_{3}$. Phys. Rev. B, 89:245113, Jun 2014.

[75] Choong H. Kim et. al. Topological Quantum Phase Transition in 5d Transition Metal Oxide $\mathrm{Na}_{2} \mathrm{IrO}_{3}$. Phys. Rev. Lett., 108(10):106401, 2012.

[76] G. Cao et. al. Evolution of magnetism in the single-crystal honeycomb iridates. Phys. Rev. B, 88(22):220414, 2013.

[77] Felix Lüpke. Master theis of Felix Lüpke, Private communication with Dr. Martin Wenderoth, 2014.

[78] Private communication with Yogesh Singh about $\mathrm{Ru}$ doped $\mathrm{Na}_{2} \mathrm{IrO}_{3}$ crystals. Technical report, 2014.

[79] Yoko Miura et. al. New-Type Phase Transition of $\mathrm{Li}_{2} \mathrm{RuO}_{3}$ with Honeycomb Structure. J. Phys. Soc. Japan, 76(3):033705, 2007.

[80] Hechang Lei et. al. Structural, magnetic, and electrical properties of $\operatorname{Li}_{2} \operatorname{Ir}_{1 \mathrm{x}} \mathrm{Ru}_{\mathrm{x}} \mathrm{O}_{3}$. Phys. Rev. B, 89(2):020409, 2014.

[81] S. Manni, Y. Tokiwa, and P. Gegenwart. Effect of nonmagnetic dilution in the honeycomb-lattice iridates $\mathrm{Na}_{2} \mathrm{IrO}_{3}$ and $\mathrm{Li}_{2} \mathrm{IrO}_{3}$. Phys. Rev. B, 89:241102, Jun 2014. 
[82] T Dey et. al. Spin-liquid behavior in $\mathrm{J}_{\text {eff }}=1 / 2$ triangular lattice compound $\mathrm{Ba}_{3} \operatorname{IrTi}_{2} \mathrm{O}_{9}$. Phys. Rev. B, 86(14):140405, 2012.

[83] Eric C. Andrade et. al. Magnetism in spin models for depleted honeycomb-lattice iridates: Spin-glass order towards percolation. arXiv: 1309.2951, page 5, 2013.

[84] Y Tokiwa and P Gegenwart. High-resolution alternating-field technique to determine the magnetocaloric effect of metals down to very low temperatures. Rev. Sci. Instrum., 82(1):013905, 2011.

[85] Lijun Zhu et. al. Universally Diverging Grüneisen Parameter and the Magnetocaloric Effect Close to Quantum Critical Points. Phys. Rev. Lett., 91(6):066404, 2003.

[86] T. Westerkamp, M. Deppe, R. Küchler, M. Brando, C. Geibel, P. Gegenwart, A. Pikul, and F. Steglich. Kondo-Cluster-Glass State near a Ferromagnetic Quantum Phase Transition. Phys. Rev. Lett., 102(20):206404, 2009.

[87] Johannes Reuther, Ronny Thomale, and Simon Trebst. Finite-temperature phase diagram of the Heisenberg-Kitaev model. Phys. Rev. B, 84(10):100406, 2011.

[88] K Foyevtsova et. al. Ab initio analysis of the tight-binding parameters and magnetic interactions in $\mathrm{Na}_{2} \mathrm{IrO}_{3}$. Phys. Rev. B, 88(3):035107, 2013.

[89] Youhei Yamaji et. al. Honeycomb Lattice Iridates $\mathrm{Na}_{2} \mathrm{IrO}_{3}$ under Strong Spin-Orbit Interaction and Electron Correlation Studied by Ab Initio Scheme. arXiv : 1402.1030, page 8, 2014.

[90] Fabien Trousselet et. al. Effects of spin vacancies on magnetic properties of the KitaevHeisenberg model. Phys. Rev. B, 84(5):054409, 2011.

[91] Cyril Martins et. al. Reduced Effective Spin-Orbital Degeneracy and Spin-Orbital Ordering in Paramagnetic Transition-Metal Oxides: $\mathrm{Sr}_{2} \mathrm{IrO}_{4}$ versus $\mathrm{Sr}_{2} \mathrm{RhO}_{4}$. Phys. Rev. Lett., 107(26):266404, 2011.

[92] Vanya Todorova et. al. Synthesis, Structural Characterization and Physical Properties of a New Member of Ternary Lithium Layered Compounds $-\mathrm{Li}_{2} \mathrm{RhO}_{3}$. Zeitschrift für Anorg. und Allg. Chemie, 637(1):37-40, 2011.

[93] Yongkang Luo et. al. $\mathrm{L}_{2} \mathrm{RhO}_{3}$ : A spin-glassy relativistic Mott insulator. Phys. Rev. B, 87(16):161121, 2013.

[94] I. I. Mazin, S. Manni, K. Foyevtsova, Harald O. Jeschke, P. Gegenwart, and Roser Valentí. Origin of the insulating state in honeycomb iridates and rhodates. Phys. Rev. B, 88(3):035115, 2013.

[95] J. A. Mydosh. Spin glasses: an experimental introduction. Taylor \& Francis, 1993.

[96] A. Ramirez et. al. Entropy Balance and Evidence for Local Spin Singlets in a KagoméLike Magnet. Phys. Rev. Lett., 84(13):2957-2960, 2000.

[97] Satoru Nakatsuji et. al. Spin disorder on a triangular lattice. Science, 309(5741):1697$700,2005$. 
[98] P Khuntia. Li- NMR as a local probe for 4d magnetism in Honeycomb lattice $\mathrm{Li}_{2} \mathrm{RhO}_{3}$ - Unpublished work. Received in private communications. Technical report, 2014.

[99] X. Zong et. al. Structure, magnetization, and NMR studies of the spin-glass compound $\left(\mathrm{Li}_{\mathrm{x}} \mathrm{V}_{1 \mathrm{x}}\right)_{3} \mathrm{BO}_{5}$ (x0.40 and 0.33). Phys. Rev. B, 76(5):054452, 2007.

[100] Yi-Zhuang You et. al. Doping a spin-orbit Mott insulator: Topological superconductivity from the Kitaev-Heisenberg model and possible application to $\left(\mathrm{Na}_{2} / \mathrm{Li}_{2}\right) \mathrm{IrO}_{3}$. Phys. Rev. B, 86(8):085145, 2012.

[101] Ki Modic et. al. A new spin-anisotropic harmonic honeycomb iridate. ArXiv: 1402.3254, 2014. 